\title{
1,3,2-Diazaphospholene-Catalyzed Reductive Cyclizations of Organohalides
}

\author{
Johannes Klett, Dr. Łukasz Woźniak and Prof. Dr. Nicolai Cramer[a]
}

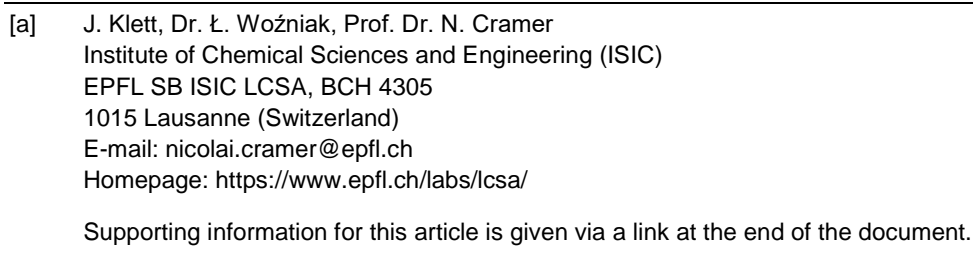

\begin{abstract}
DAP-Hs) are highly nucleophilic organic hydrides serving as main-group catalysts for a range of attractive transformations. DAP hydrides can act as stoichiometric hydrogen atom transfer agents in radical reactions. Herein, we report a DAP-catalyzed reductive radical cyclization of a broad range of aryl and alkyl halides under mild conditions. The pivotal DAP catalyst turnover was achieved by a DBU-assisted $\sigma$ bond metathesis between the formed DAP halide and HBpin rapidly regenerating DAP-H. The transformation is significantly accelerated by irradiation with visible light. Mechanistic investigations indicate that visible light irradiation leads to the formation of DAP dimers which are in equilibrium with the DAP radicals accelerating the cyclization. The direct use of $(\mathrm{DAP})_{2}$ enabled a catalytic protocol in the absence of light
\end{abstract}

Discovered in the 1980s, 1,3,2-diazaphospholenes (DAPs) ${ }^{[1]}$ represent a class of electron-rich heterocycles with a great application potential as versatile main-group catalysts. ${ }^{[2]}$ For, instance, diazaphospholene hydrides (DAP-Hs) are powerful nucleophiles and weakly basic organic hydride donors. ${ }^{[3]}$ They are very capable to reduce carbonyl compounds ${ }^{[4]}$ and in conjugate fashion $\alpha, \beta$-unsaturated amides, ${ }^{[5]}$ esters, ${ }^{[5 b, 6]}$ ketones $^{[5]}$ and acids. $^{[7]}$ A $\sigma$-bond metathesis between the alkoxy-substituted DAPs and pinacol borane (HBpin) regenerating DAP-H rendered these processes catalytic. ${ }^{[4]}$ Reports by Kinjo and Speed utilized metathesis between the $\mathrm{P}-\mathrm{N}$ and $\mathrm{B}-\mathrm{H}$ bonds employing HBpin and ammonia borane to enable catalytic reductions of azobenzenes $^{[8]}$ and imines. ${ }^{[5 b, 9]}$ Cheng et al. demonstrated the cleavage of the P-F bond in DAP fluorides by phenylsilane enabling hydrodefluorination of trifluoromethyl alkenes ${ }^{[10]}$ and polyfluoro arenes. ${ }^{[11]}$ The reactivity of DAP-Hs is not limited to the two electron transfer processes. ${ }^{[12]}$ These electron-rich heterocycles can engage in reductions of alkyl and aryl halides ${ }^{[13]}$ and $\alpha$-carboxy ketones ${ }^{[14]}$ via radical pathways. However, radical processes that can use catalytic amounts of DAPs are very scarce. A single report of deoxygenation of $\alpha$-carboxy ketones employs diazaphosphinane as catalyst and AIBN as an initiator. ${ }^{[14]}$ It capitalizes on the $\sigma$-bond metathesis between the $\mathrm{P}$ $\mathrm{O}$ and $\mathrm{B}-\mathrm{H}$ bonds for the catalyst's regeneration. In contrast to the DAP-F, the related halide bearing DAP-X $(X=I, B r, C l)$ do not undergo a $\sigma$-bond metathesis with borane or silane reagents. This represent a relevant reactivity gap hampering the closure of catalytic cycles. To exploit the full potential of DAPs in radical chemistry with organohalides, it is essential to render the transformations catalytic.

Radical cyclizations of organohalides across olefins are highly useful transformations to access diverse cyclic skeletons. ${ }^{[15]}$ Typically, reductive cyclization of organohalides operating by radical mechanism require stoichiometric amounts of toxic organostannanes ${ }^{[16]}$ and radical initiators such as e.g. $\mathrm{AIBN}{ }^{[17]}$ or $\mathrm{Et}_{3} \mathrm{~B} / \mathrm{O}_{2} \cdot{ }^{[18]}$ Advances in photochemical methods provided some alternatives including the use of metal- and organic photocatalysts ${ }^{[19]}$ as well as electron-donor reagents. ${ }^{[20]}$ The high affinity of DAPs towards organohalides makes them very attractive for activating this broad compounds class. ${ }^{[21]}$ Speed demonstrated the functionalization of organo iodides and bromides. ${ }^{[22]}$ However, catalytic variants of these processes remain so far elusive. A rapid and efficient regeneration of the DAP-H from DAP-X under mild conditions would be an essential gateway to catalytic transformations with organohalide substrate. Herein, we report an efficient Lewis-base promoted regeneration of DAP-H from DAP-X and its application for reductive radical cyclizations of aryl and alkyl halides under catalytic conditions.

a) Pivotal regenation of DAP-H for catalytic applications
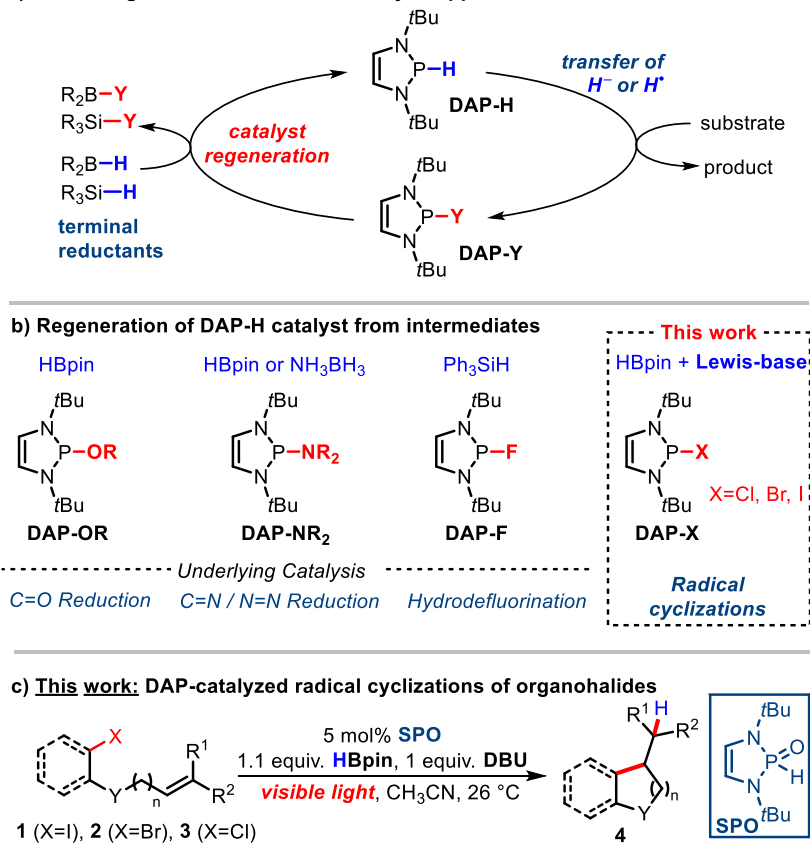

Scheme 1. a) DAPs in catalysis: Regeneration of DAP-H to close catalytic cycles. b) Landscape of reduction options for DAP-Y into DAP-H and the underlying catalytic processes $\mathrm{C}$ ) DAP-catalyzed reductive radical cyclization of organohalides.

To explore the ways to close the catalytic cycle, we first investigated the possibility to reliably regenerate the pivotal DAP-H species from the formed DAP-I of the stoichiometric process. Mixing DAP-I and HBpin in $\mathrm{CD}_{3} \mathrm{CN}$ does not lead to any 
meaning formation of DAP-H after $16 \mathrm{~h}$ (Scheme 2). We hypothesized that activation of the borane by a suitable Lewis base ${ }^{[23]}$ could facilitate the $\sigma$-bond metathesis between the DAP-I and HBpin. To our delight, adding DBU (1,8diazabicyclo[5.4.0]undec-7-ene) to the DAP-I/HBpin mixture in $\mathrm{CD}_{3} \mathrm{CN}$ triggered the quantitative generation of DAP-H in less than ten minutes. DMAP or $N$-methyl imidazole were as well competent (see SI for details).

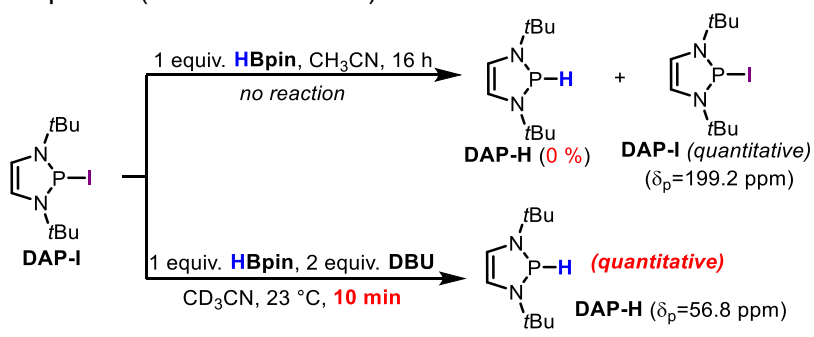

Scheme 2. Fast regeneration of DAP-H with the DBU/HBpin system.

With the rapid DAP-H regeneration from DAP-X solved by the DBU-assisted $\sigma$-bond metathesis, we turned our efforts towards a fully catalytic transformation for the cyclization of aryl iodide 1a and aryl bromide 2a (Table 1, for a comprehensive optimization study, see SI). Employing $5 \mathrm{~mol} \%$ of the robust and conveniently usable secondary phosphine oxide $\mathbf{S P O}^{[24]}$ as the pre-catalyst in combination with the HBpin/DBU mixture in MeCN, gave cyclization product $4 a$ in $23 \%$ yield (entry 1). This corresponds to over four catalyst turnovers. During our optimization studies, we noticed that the reaction rate and progress is highly sensitive to light. While still taking place under the careful exclusion of light (entry 2), the yield of $\mathbf{4 a}$ almost quadrupled when irradiating the reaction mixture with white LEDs (entry 3). This effect was even more pronounced with aryl bromide 2a. However, no reaction took place in the absence of light (entry 4). The use of white LEDs restored some reactivity giving $\mathbf{4 a}$ in $12 \%$ yield (entry 5 ). Switching to a more powerful a Kessil lamp $(427 \mathrm{~nm})$ allowed to increase the yield of $\mathbf{4 a}$ to $91 \%$ (entry 6). Control experiments omitting SPO, DBU and HBpin showed their indispensable role in the transformation (entries 7-9). Aryl chloride $3 \mathbf{a}$ did not react under the current conditions (entry 10).

Table 1. Optimization of the DAP-catalysed cyclization of 1-2a. ${ }^{[a]}$

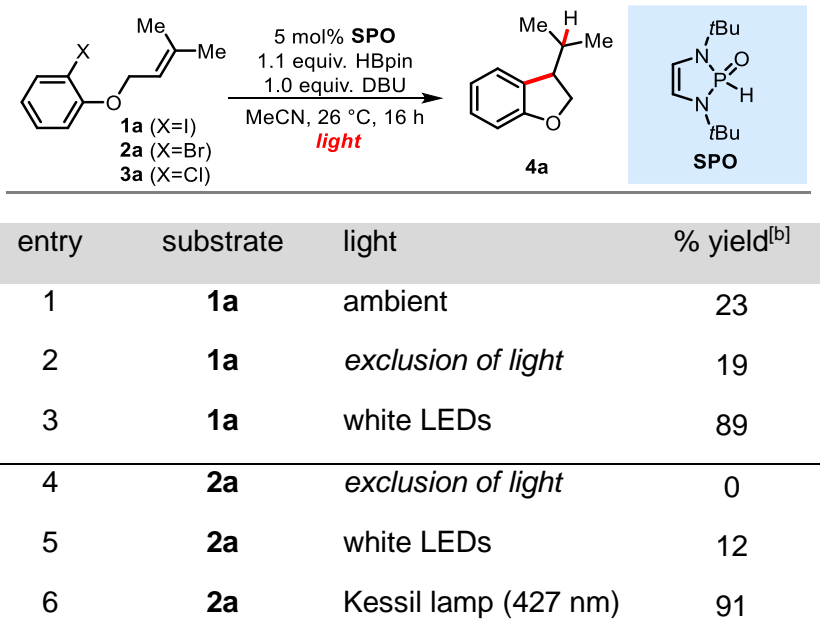

$\begin{array}{llll}7^{[\mathrm{c}]} & \mathbf{2 a} & \text { Kessil lamp }(427 \mathrm{~nm}) & 0 \\ 8^{[\mathrm{d}]} & \mathbf{2 a} & \text { Kessil lamp }(427 \mathrm{~nm}) & 5 \\ 9^{[\mathrm{e}]} & \mathbf{2 a} & \text { Kessil lamp }(427 \mathrm{~nm}) & 0 \\ 10 & \mathbf{3 a} & \text { Kessil lamp }(427 \mathrm{~nm}) & 0\end{array}$

[a] Conditions: $0.1 \mathrm{mmol}$ 1a, $5 \mu \mathrm{mol}$ SPO, $0.11 \mathrm{mmol} \mathrm{HBpin,} 0.1 \mathrm{mmol}$ DBU, 0.1 $\mathrm{M}(0.8 \mathrm{M}$ for $2 \mathrm{a}$ and $3 \mathrm{a})$ in MeCN at $26^{\circ} \mathrm{C}$ for $16 \mathrm{~h}$, [a] Yields determined by ${ }^{1} \mathrm{H}-\mathrm{NMR}$ with an internal standard; [c] no SPO [d] no DBU; [d] no HBpin.

Next, we evaluated the scope of the DAP-catalyzed reductive cyclization of a broad set of substituted aryl halide substrates (Scheme 3). Dihydrobenzofurane $\mathbf{4 a}$ was isolated in excellent yields (92\% from 1a and $96 \%$ from 2a). The transformation is tolerant to potential reactive functional groups like methyl esters, free phenols and carboxylic acids reliably delivering products $\mathbf{4 b - 4 g}$. Notably, a switch from the Kessil lamp $(427 \mathrm{~nm})$ to white LEDs allowed for a selective cyclization originating from the aryl iodide moiety of $\mathbf{1 j}$ in the presence of the adjacent bromide substituent in $97 \%$ yield. Along the same lines, the reaction of aryl bromide $\mathbf{2} \mathrm{h}$ cleanly proceeded in the presence of an adjacent chloride moiety under standard conditions. The reaction of the bromo pyridine $\mathbf{2} \mathbf{i}$ cyclized to product $4 \mathbf{i}$ without DAP-catalyzed reduction of its pyridine core. ${ }^{[25]}$ Aryl bromides bearing different alkenyl tethers reacted well. For instance, styrene $\mathbf{2 k}$ underwent cyclization in $86 \%$ yield. The nature of the olefin acceptor portion was as well modifiable (4I-4n). Substrates which pass through $1^{\circ}$ or $2^{\circ}$ alkyl radical intermediates reacted in reduced yields (4I and $\mathbf{4 m}$ ). Besides the formation of the fivemembered rings, the process enabled the 6-exo-trig cyclizations as demonstrated for 4-isopropylchromane 4o. Aniline substrates were readily converted into functionalized indolines $4 p-4 r$. Next, we explored the potential of alkyl halide substrates for the cyclization. In this respect, iodo acetals $1 \mathrm{~s}$ and bromo $2 \mathrm{~s}$ smoothly reacted providing bicyclic product $4 \mathbf{s}$. Primary alkyl bromides $\mathbf{2 u}$ and $\mathbf{2 t}$ cyclized to the corresponding tetrahydrofuran $\mathbf{4 u}$ and cyclopentane $\mathbf{4 t}$ in moderate yields. The cyclization of substituted alkenyl amines led to pyrrolidines $\mathbf{4 v - 4 w}$ in good yield. A secondary alkyl bromide analogue formed disubstituted pyrrolidine $4 y$ in 91\% yield and 65:35 dr. Noteworthy, substrates bearing a trichloroalkyl group engaged in the transformation providing dichloro-substituted pyrrolidine $\mathbf{4 z}$ and piperidine $\mathbf{4 z a}$ in $82 \%$ and $69 \%$ yield, respectively. 


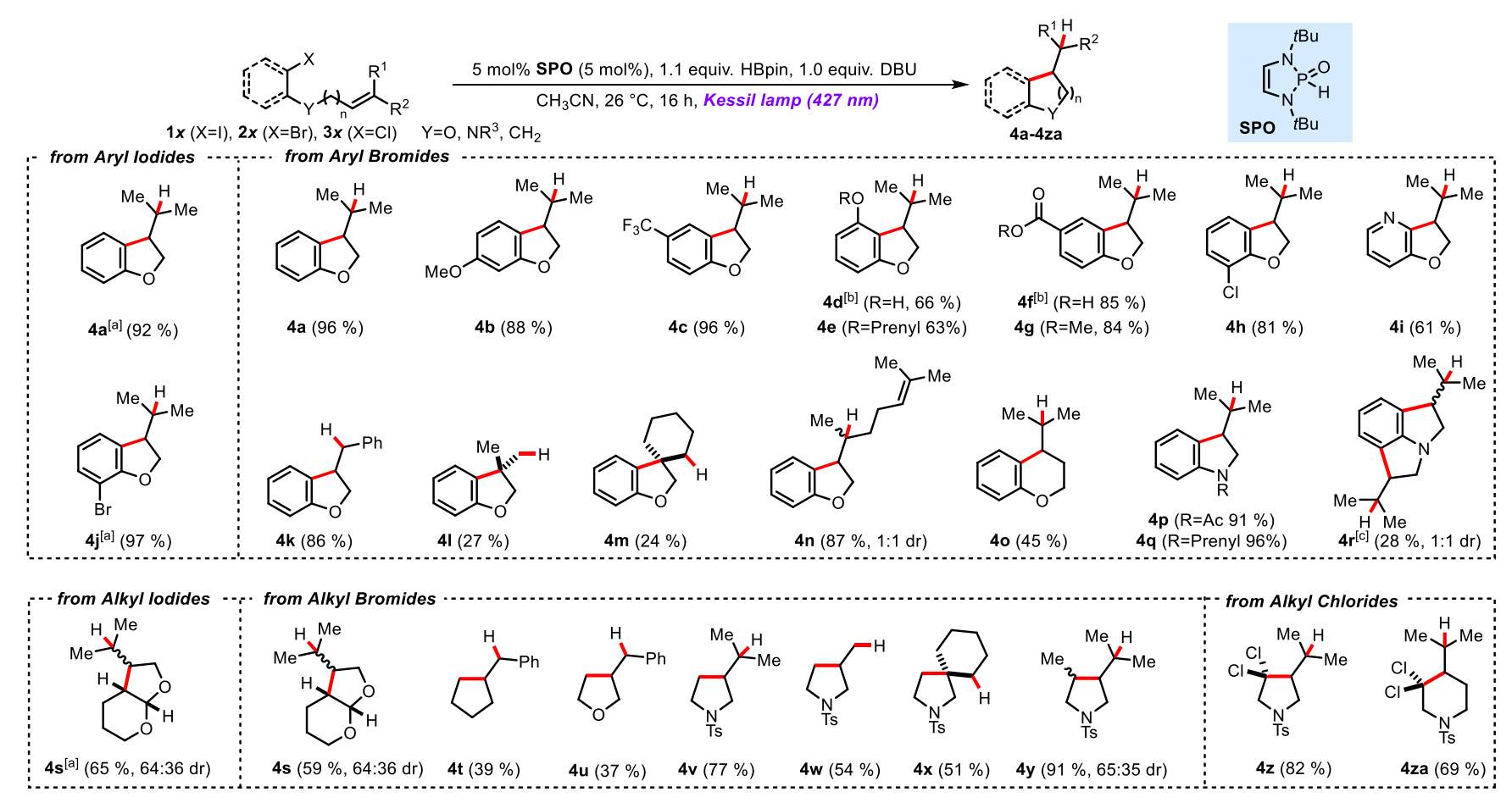

Scheme 3: Scope of the DAP-catalyzed cyclization of organohalides. Conditions: $0.20 \mathrm{mmol} \mathbf{1} \boldsymbol{x}-\mathbf{3 x}, 10 \mu \mathrm{mol}$ SPO, $0.22 \mathrm{mmol} \mathrm{HBpin}, 0.20 \mathrm{mmol}$ DBU in MeCN $(\mathbf{1} \boldsymbol{x}=0.2 \mathrm{M}, \mathbf{2} \boldsymbol{x}-\mathbf{3} \boldsymbol{x}=0.8 \mathrm{M})$ in MeCN at $26^{\circ} \mathrm{C}$ for $16 \mathrm{~h}$. [a] white LEDs instead of the Kessil lamp $427 \mathrm{~nm}$. [b] 2.5 equiv. HBpin. [c] $10 \mathrm{~mol} \% \mathrm{SPO}, 2.2 \mathrm{equiv}$. HBpin and 2.0 equiv. DBU.

We next conducted mechanistic investigations to better understand the effect the light plays in this transformation. The stoichiometric reactions indicate that the cyclizations of $\mathbf{1 a}$ and $\mathbf{2 a}$ take as well place in the dark. However, irradiation by a Kessil lamp $(427 \mathrm{~nm})$ accelerates the transformations (Scheme 4). In contrast, the catalytic reaction with substrate $2 \mathrm{a}$ occurs only under irradiation. Aryl bromide $\mathbf{2 a}$ still cyclized to $\mathbf{4 a}$ in the dark, albeit with poor efficiency. The direct reaction of DAP-H and $\mathrm{CCl}_{4}$ does not require activation by strong light sources affording $\mathbf{D A P}-\mathbf{C l}$ and a mixture of chloromethanes $\mathrm{CH}_{m} \mathrm{Cl}_{4-m}(\mathrm{~m}=0-3) .{ }^{[21 b]}$ This $\mathrm{P}-$ $\mathrm{H} / \mathrm{C}-\mathrm{Cl}$ bond metathesis proceeds eventually through a radical mechanism. Substrate $\mathbf{3 z}$ was exposed to 1 equiv. of DAP-H under exclusion of light yielding $75 \%$ of product $\mathbf{4 z}$. Attempts to inhibit the hypothesized radical mechanism by the addition of 20 mol\% TEMPO were futile. TEMPO rapidly reacts with DAP-H and after consumption of all added TEMPO, the remaining amount of DAP-H produced $\mathbf{4 z}$ in $54 \%$ yield. To gain mechanistic insight of the light-enhanced reaction, we first identified which species absorb light in the visible spectrum. The absorption spectra of DAPs and 2a indicates that only DAP-H and DAP-Br absorb light at wavelengths above $400 \mathrm{~nm}$, which is an emission tail of the Kessil lamp (427 nm). No ground state associations between the DAPs and 2a were found (see SI). Based on this information, two scenarios in which visible light accelerates the cyclization of $\mathbf{2 a}$ are plausible. In scenario I, a photoexcitation of DAP-H triggers a SET from the excited DAP-H ${ }^{\star}$ to $\mathbf{2 a}$. Scenario II involves a photoexcitation of DAP-H leading to a 1,3,2-diazaphospholene radical (DAP•). On the basis of electrochemical and spectroscopic measurements, we estimated the redox potential of the excited DAP-H* is $-3.14 \mathrm{~V}$ (vs. $\mathrm{Fc}^{+} / \mathrm{Fc}$ in $\left.\mathrm{MeCN}\right)$, indicating that a SET between $\mathbf{D A P}-\mathrm{H}^{*}$ and $\mathbf{2 a}\left(\mathrm{E}_{\mathrm{red}} \mathbf{2 a} / \mathbf{2} \mathbf{a}^{-}=-3.36 \mathrm{~V} v \mathrm{v}\right.$. $\mathrm{Fc}^{+} / \mathrm{Fc}$ in $\mathrm{MeCN}$ ) is endergonic (see $\mathrm{SI}$ for details). Next, we examined the generation of DAP• radical species by excitation of DAP-H. We hypothesized that visible light from the Kessil lamp (427 nm) could trigger a reductive dimerization of DAP-H. This was previously reported by Gudat et al. using UV light from a medium-pressure Hg-lamp. ${ }^{[26]}$ The resulting (DAP) 2 species, described as a weakly $\sigma$-bonded dimer, would dissociate in solution to give the persistent $7 \pi$-radical DAP• species. ${ }^{[27]}$ Indeed, ${ }^{31} \mathrm{P}-\mathrm{NMR}$ analysis confirmed formation of (DAP) ${ }_{2}$ from DAP-H upon irradiation by a Kessil lamp $(427 \mathrm{~nm})$ (Scheme 5). This evidence supports the suggestion of an initiation of a radical chain process through the immediacy of (DAP) 2 .

I) Light influence on the stoichiometric cyclization

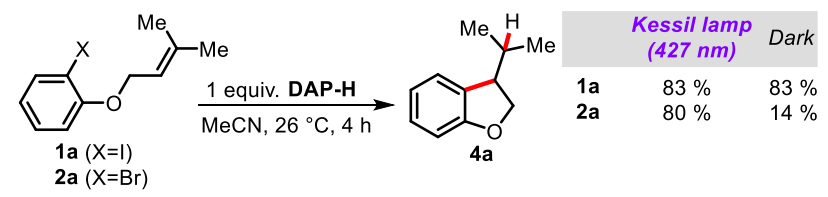

II) Stoichiometric cyclization of $3 z$ in the dark

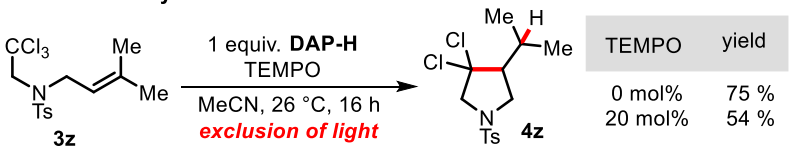

III) Absorption spectra

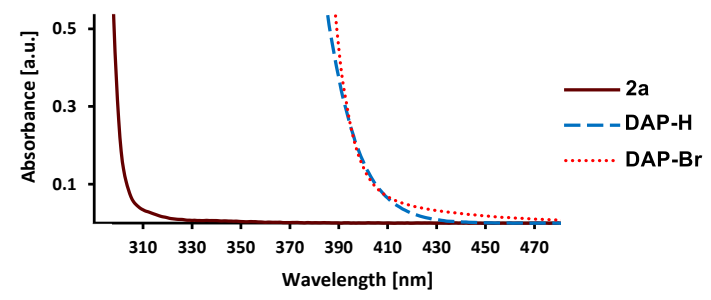

Scheme 4. Evaluation of the light influence or TEMPO on the cyclization efficiency and absorption spectra of the reaction components.

Next, we tested the influence of (DAP) ${ }_{2}$ on the cyclizations reaction rate of $\mathbf{2 a}$ with one equivalent of DAP-H in the dark. 
Notably, already 2 mol\% of (DAP) 2 remarkably accelerates the cyclization. This observation clearly supports the outlined scenario II. Both findings let to the hypothesis that an exchange of the SPO catalyst by (DAP) 2 would enable a catalytic process without the visible light activation. Remarkably, $5 \mathrm{~mol} \%$ (DAP) ${ }_{2}$ promoted a catalytic reaction in the absence of light forming $4 a$ in $54 \%$ yield.

l) Reductive dimerization of DAP-H to $(\mathrm{DAP})_{2}$ under visible light irradiation

$$
\begin{aligned}
& \underset{\mathrm{CD}_{3} \mathrm{CN}, 26^{\circ} \mathrm{C}, 5 \mathrm{~h}}{\stackrel{\text { Kessil lamp }(427 \mathrm{~nm})}{t}} \\
& \delta_{p} \text { (quantitative) }
\end{aligned}
$$

II) Influence of $(\mathrm{DAP})_{2}$ on the reaction rates of the cyclization of $2 \mathrm{a}$
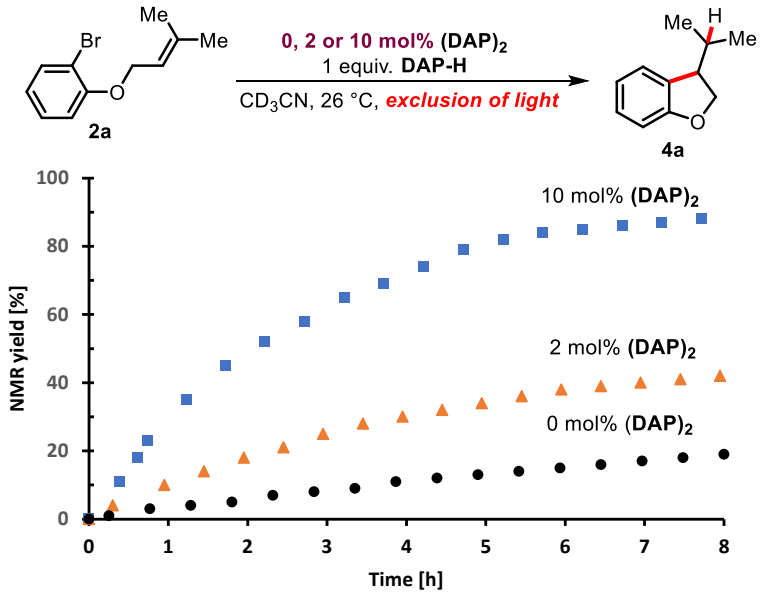

III) The use of $(\mathrm{DAP})_{2}$ as competent catalyst for the dark reaction

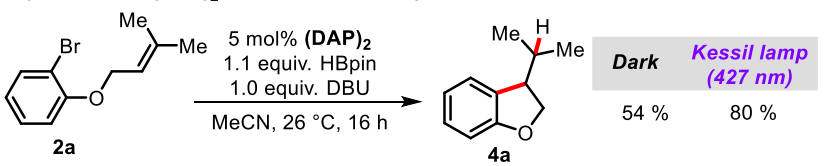

Scheme 5. Illustration of the role of (DAP) $)_{2}$ in the DAP-catalyzed cyclizations.

Further mechanistic experiments comprised initial-rate kinetic studies of the model reaction across a range of concentrations for each reaction component under visible light irradiation (see SI for details). A first-order dependence was inferred for DAP-H and a half order for substrate $\mathbf{2 a}$, supporting a radical chain mechanism. The zeroth-order dependence on the concentration of HBpin and of DBU indicate that the regeneration of the DAP-H is not a turnover limiting step. Moreover, the reaction rates increase with the increasing light intensity (see $\mathrm{SI}$ ). Deuterium labelling studies with 1a using DAP-D in $\mathrm{CH}_{3} \mathrm{CN}$ or DAP-H in $\mathrm{CD}_{3} \mathrm{CN}$ confirm the origin of the hydrogen atom of $4 \mathrm{a}$ from the catalyst.

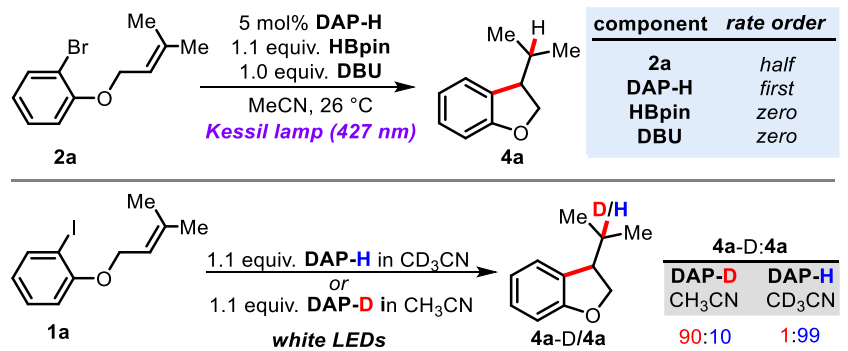

Scheme 6. Initial-rate kinetics for the DAP-catalyzed cyclization and deuterium labelling studies.
Taking all mechanistic experiments into account, the catalytic cycle can be portrayed (Scheme 7). With SPO as the pre-catalyst, the process is initiated by the reduction with HBpin forming DAP-H. Visible light irradiation converts DAP-H to $(D A P)_{2}$. The dissociation equilibrium of (DAP) ${ }_{2}$ into two molecules of DAP. initiates a radical chain process by bromine atom abstraction from 2 a. $^{[28]}$ The resulting aryl radical $I$ adds across the $\mathrm{C}=\mathrm{C}$ bond in a 5-exo-trig fashion forming radical species II. In turn, II abstracts the hydrogen atom from DAP-H delivering product $\mathbf{4 a}$ and DAP. Enabled by DBU, DAP-Br is converted back to DAP-H with HBpin. Employing (DAP) 2 as catalyst allows entering the catalytic cycle bypassing the light activation step. However, the reduced yield of the (DAP) $)_{2}$-catalyzed reaction in the dark indicates that light can heal the catalytic cycle by regeneration DAP• after radical chain terminations.

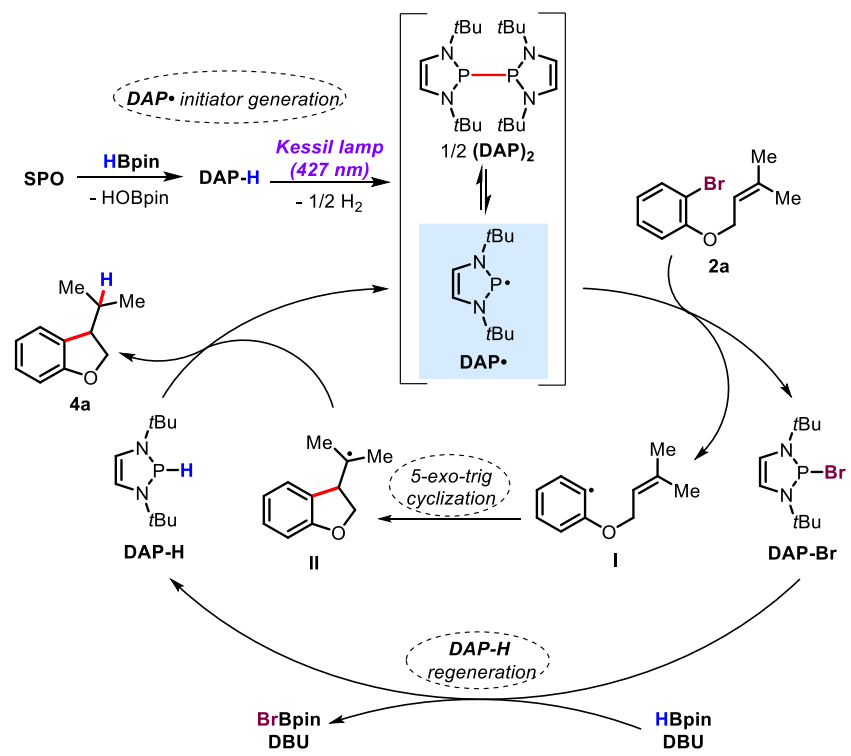

Scheme 7. Proposed mechanism of the DAP-catalyzed cyclization of organohalides.

In summary, we developed a DAP-catalyzed reductive radical cyclization of organohalides. The DAP catalyst turnover was achieved by implementation of a DBU-assisted $\sigma$-bond metathesis between DAP-X $(X=\mathrm{I}, \mathrm{Br}, \mathrm{Cl})$ and HBpin providing a fast regeneration of DAP-H. The transformation is significantly accelerated by the irradiation with visible light. The developed process allowed the efficient reductive cyclizations of a broad range of aryl and alkyl halides under mild and convenient conditions. Detailed mechanistic investigations revealed that visible light leads to the formation of (DAP) ${ }_{2}$ which is in equilibrium with DAP- accelerating the cyclization. The direct use (DAP) ${ }_{2}$ enabled a catalytic protocol in the absence of light. These findings will serve as blueprint and accelerator for the further developments of DAP-catalyzed radical processes.

\section{Acknowledgements}

This work is supported by the EPFL and the NCCR Catalysis. This publication was created as part of NCCR Catalysis (grant number 180544), a National Centre of Competence in Research funded by the Swiss National Science Foundation. 
Keywords: DAP Catalysis • Phosphorous • Cyclization • Radical Chemistry

[1] a) A. Schmidpeter, K. Karaghiosoff, Z. Naturforsch. B 1981, 36, 1273; b) K. Karaghiosoff, J. P. Majoral, A. Meriem, J. Navech, A. Schmidpeter, Tetrahedron Lett. 1983, 24, 2137; c) A. M. Kibardin, Y. B. Mikhailov, T. V. Gryaznova, A. N. Pudovik, Bull. Acad. Sci. USSR Div. Chem. Sci. 1986, 35, 878; d) W. B. Jennings, D. Randall, S. D. Worley, J. H. Hargis, J. Chem. Soc., Perkin Trans. 2 1981, 4, 1411.

[2] a) J. H. Reed, J. Klett, C. Steven, N. Cramer, Org. Chem. Front. 2020, 7, 3521; b) A. W. H. Speed, Chem. Soc. Rev. 2020, 49, 8335; c) D. M. C Ould, R. L. Melen, Chem. Eur. J. 2020, 26, 9835; d) J. Zhang, J.-D. Yang J.-P. Cheng, Natl. Sci. Rev. 2021, 8, DOI 10.1093/nsr/nwaa253.

[3] J. Zhang, J. D. Yang, J. P. Cheng, Angew. Chem. Int. Ed. 2019, 58, 5983.

[4] C. C. Chong, H. Hirao, R. Kinjo, Angew. Chem. Int. Ed. 2015, 54, 190.

[5] a) S. Miaskiewicz, J. H. Reed, P. A. Donets, C. C. Oliveira, N. Cramer, Angew. Chem. Int. Ed. 2018, 57, 4039; b) M. R. Adams, C. H. Tien, B. S N. Huchenski, M. J. Ferguson, A. W. H. Speed, Angew. Chem. Int. Ed. 2017, 56, 6268.

[6] C. C. Chong, B. Rao, R. Kinjo, ACS Catal. 2017, 7, 5814

[7] J. H. Reed, N. Cramer, ChemCatChem 2020, 12, 4262.

[8] C. C. Chong, H. Hirao, R. Kinjo, Angew. Chem. Int. Ed. 2014, 53, 3342.

[9] a) M. R. Adams, C. H. Tien, R. McDonald, A. W. H. Speed, Angew. Chem. Int. Ed. 2017, 56, 16660; b) T. Lundrigan, E. N. Welsh, T. Hynes, C. H. Tien, M. R. Adams, K. R. Roy, K. N. Robertson, A. W. H. Speed, J. Am. Chem. Soc. 2019, 141, 14083.

[10] J. Zhang, J.-D. Yang, J.-P. Cheng, Nat. Commun. 2021, 12, 2835

[11] J. Zhang, X. Zhao, J.-D. Yang, J.-P. Cheng, J. Org. Chem. 2022, 87, 294.

[12] J. Zhang, J. D. Yang, J. P. Cheng, Chem. Sci. 2020, 11, 3672.

[13] J. Zhang, J. D. Yang, J. P. Cheng, Chem. Sci. 2020, 11, 4786.

[14] J. Zhang, J. D. Yang, J. P. Cheng, Chem. Sci. 2020, 11, 8476.

[15] a) B. Giese, B. Kopping, T. Göbel, J. Dickhaut, G. Thoma, K. J. Kulicke, F. Trach, in Org. React., John Wiley \& Sons, Inc., Hoboken, NJ, USA 1996, pp. 301-856; b) R. Lekkala, R. Lekkala, B. Moku, K. P. Rakesh, H.-L. Qin, Eur. J. Org. Chem. 2019, 2019, 2769.

[16] a) M. Braun, R. Veith, G. Moll, Chem. Ber. 1985, 118, 1058; b) I. J. Boyer, Toxicology 1989, 55, 253; c) T. Yamakawa, H. Kinoshita, K. Miura, J. Organomet. Chem. 2013, 724, 129.

[17] a) S. R. Graham, J. A. Murphy, D. Coates, Tetrahedron Lett. 1999, 40, 2415; b) H. Yorimitsu, H. Shinokubo, K. Oshima, Chem. Lett. 2000, 29 104 ; c) K. Orito, S. Uchiito, Y. Satoh, T. Tatsuzawa, R. Harada, M. Tokuda, Org. Lett. 2000, 2, 307.

[18] a) T. Nakamura, H. Yorimitsu, H. Shinokubo, K. Oshima, Synlett 1999, 1999, 1415; b) C. Ollivier, P. Renaud, Chem. Rev. 2001, 101, 3415; c) K. Fujita, T. Nakamura, H. Yorimitsu, K. Oshima, J. Am. Chem. Soc. 2001, 123, 3137; d) N. Hayashi, I. Shibata, A. Baba, Org. Lett. 2004, 6, 4981.

[19] a) H. Kim, C. Lee, Angew. Chem. Int. Ed. 2012, 51, 12303; b) G. Revol, T. McCallum, M. Morin, F. Gagosz, L. Barriault, Angew. Chem. Int. Ed. 2013, 52, 13342; c) C. Yang, F. Mehmood, T. L. Lam, S. L.-F. Chan, Y. Wu, C.-S. Yeung, X. Guan, K. Li, C. Y.-S. Chung, C.-Y. Zhou, T. Zou, C.M. Che, Chem. Sci. 2016, 7, 3123; d) B. Schweitzer-Chaput, M. A Horwitz, E. de Pedro Beato, P. Melchiorre, Nat. Chem. 2019, 11, 129.

[20] a) C. A. D. Caiuby, A. Ali, V. T. Santana, F. W. de S. Lucas, M. S. Santos, A. G. Corrêa, O. R. Nascimento, H. Jiang, M. W. Paixão, RSC Adv. 2018, 8, 12879; b) K. Liang, N. Li, Y. Zhang, T. Li, C. Xia, Chem. Sci. 2019, 10 3049 .

[21] a) S. Burck, D. Gudat, M. Nieger, Angew. Chem. Int. Ed. 2004, 43, 4801; b)S. Burck, D. Gudat, M. Nieger, W. W. Du Mont, J. Am. Chem. Soc 2006, 128, 3946

[22] B. S. N. Huchenski, K. N. Robertson, A. W. H. Speed, Eur. J. Org. Chem. 2020, 2020, 5140

[23] a) A. Staubitz, A. P. M. Robertson, M. E. Sloan, I. Manners, Chem. Rev. 2010, 110, 4023; b) M.-A. Légaré, M.-A. Courtemanche, F.-G. Fontaine, Chem. Commun. 2014, 50, 11362; c) C. Das Neves Gomes, E. Blondiaux, P. Thuéry, T. Cantat, Chem. Eur. J. 2014, 20, 7098.

[24] T. Lundrigan, C. H. Tien, K. N. Robertson, A. W. H. Speed, Chem. Commun. 2020, 56, 8027.
[25] a) B. Rao, C. C. Chong, R. Kinjo, J. Am. Chem. Soc. 2018, 140, 652; b) T. Hynes, E. N. Welsh, R. McDonald, M. J. Ferguson, A. W. H. Speed Organometallics 2018, 37, 841 .

[26] O. Puntigam, L. Könczöl, L. Nyulászi, D. Gudat, Angew. Chem. Int. Ed 2015, 54, 11567.

[27] R. Edge, R. J. Less, E. J. L. McInnes, K. Müther, V. Naseri, J. M. Rawson, D. S. Wright, Chem. Commun. 2009, 1691.

[28] An alternative mechanism involving SET from DAP• $\left(E_{\text {red }} \mathbf{D A P}+/ \mathbf{D A P} \bullet=\right.$ $-1.76 \mathrm{~V} v$ s. $\mathrm{Fc}^{+} / \mathrm{Fc}$ in $\left.\left.\mathrm{MeCN}\right)\right)$ to $2 \mathrm{a}\left(\mathrm{E}_{\mathrm{red}} \mathbf{2} \mathbf{a} / \mathbf{2 \mathbf { a } ^ { - }}=-3.36 \mathrm{~V}\right.$ vs. $\mathrm{Fc}^{+} / \mathrm{Fc}_{\mathrm{c}}$ in $\mathrm{MeCN}$ ) is thermodynamically prohibited (see SI for details) 


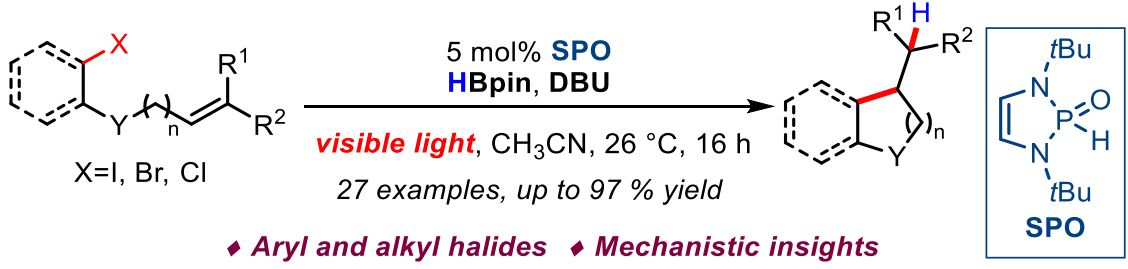

- Aryl and alkyl halides • Mechanistic insights
J. Klett, Ł. Woźniak, N. Cramer*

[Page No. - Page No.

1,3,2-Diazaphospholene-Catalyzed Reductive Cyclizations of Organohalides

1,3,2-diazaphospholenes hydrides (DAP-H) are shown as efficient catalysts for reductive radical cyclization of aryl and alkyl halides under mild conditions and accelerated by irradiation with visible light. The pivotal DAP catalyst turnover was achieved by a DBUassisted $\sigma$-bond metathesis between the formed DAP halide and HBpin rapidly regenerating DAP-H. 


\section{Supporting Information}

\section{1,3,2-Diazaphospholene-Catalyzed Reductive Cyclizations of Organohalides}

Johannes Klett, Dr. Łukasz Woźniak and Prof. Dr. Nicolai Cramer*

Table of Contents

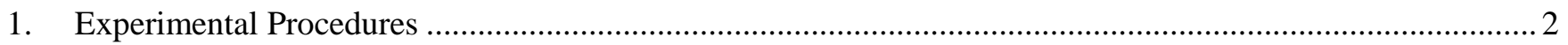

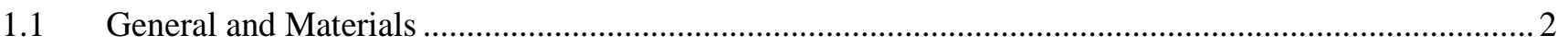

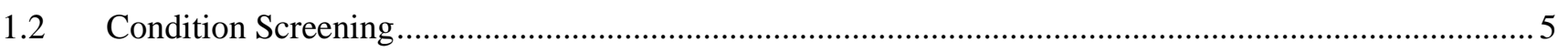

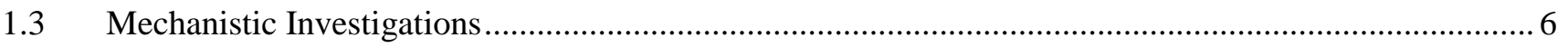

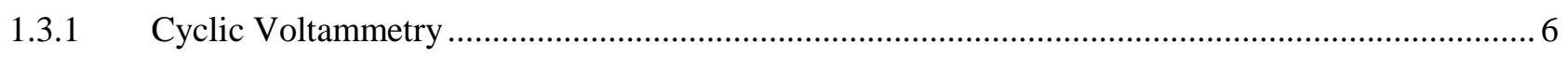

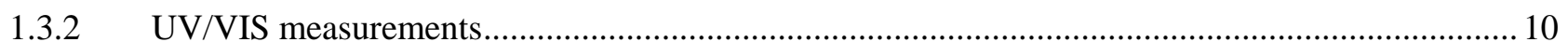

1.3.3 Formation of DAP-H from DAP-I with DBU as an Activator.................................................... 13

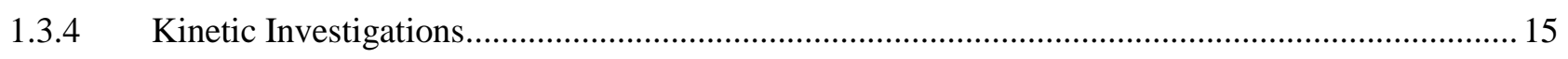

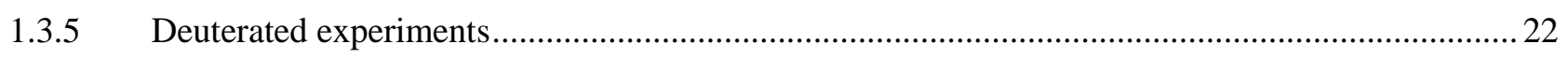

1.3.6 Formation of $(\mathrm{DAP})_{2}$ from DAP-H under Irradiation .............................................................. 24

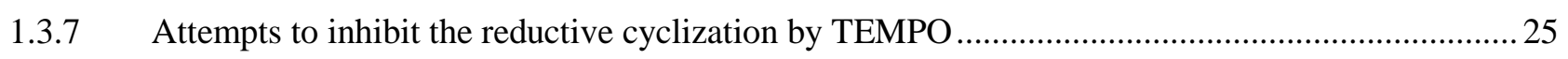

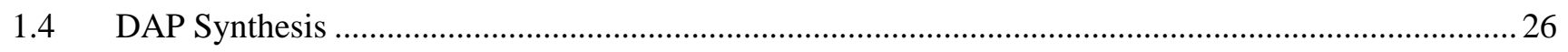

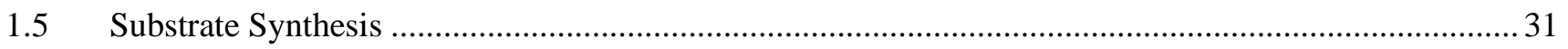

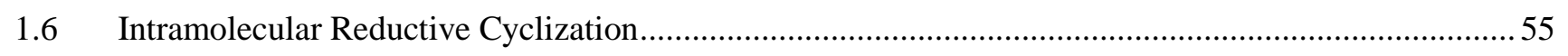

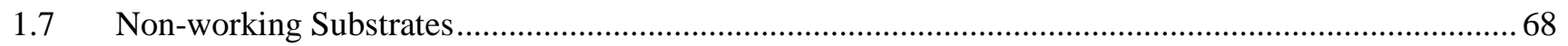

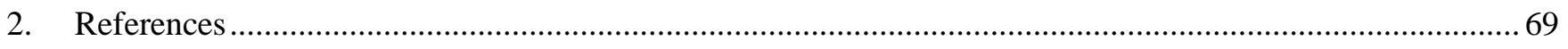

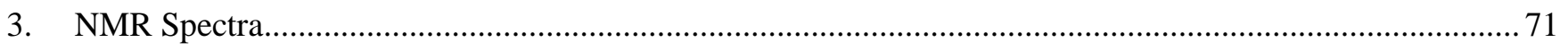




\section{Experimental Procedures}

\subsection{General and Materials}

\section{Experimental Techniques}

All reactions were carried out under an atmosphere of nitrogen in oven-dried glassware with magnetic stirring inside a glove box or using Schlenk technique, unless otherwise indicated. Chemicals were used as obtained from the suppliers unless otherwise indicated.

\section{Drying, Degassing and Purification of Solvents}

$\mathrm{CH}_{2} \mathrm{Cl}_{2}$, THF and $\mathrm{MeCN}$ were purified by an Innovative Technology Solvent Delivery System. $n$-Hexane was distilled over $\mathrm{CaH}_{2}$ under nitrogen atmosphere. Before being transferred into a nitrogen-filled glove box to be stored over $4 \AA$ molecular sieves, all solvents were degassed via freeze-pump-thaw technique. 1,8diazabicyclo(5.4.0)undec-7-ene (DBU) was distilled over $\mathrm{CaH}_{2}$ under nitrogen atmosphere, degassed via freezepump-thaw technique and stored inside the glovebox.

\section{Flash Chromatography}

Flash chromatography was performed with SiliaFlash silica gel P60 (40-63 $\mu \mathrm{m}$ grade). Analytical thin-layer chromatography was performed with commercial glass plates coated with $0.25 \mathrm{~mm}$ silica gel (E. Merck, Kieselgel $60 \mathrm{~F}_{254}$ ). Compounds were either visualized under UV-light at $254 \mathrm{~nm}$ and/or by dipping the plates in $\mathrm{KMnO}_{4}$ stain: $\left(\mathrm{KMnO}_{4}(3.0 \mathrm{~g}), \mathrm{Na}_{2} \mathrm{CO}_{3}(20 \mathrm{~g})\right.$, aq $\mathrm{NaOH}$ solution $(5 \mathrm{wt} \%, 5.0 \mathrm{~mL})$ in $\left.\mathrm{H}_{2} \mathrm{O}(300 \mathrm{~mL})\right)$ or Vanillin stain: vanillin $(10 \mathrm{~g})$ and $\mathrm{H}_{2} \mathrm{SO}_{4}$ (conc., $\left.1 \mathrm{~mL}\right)$ in $\mathrm{EtOH}(250 \mathrm{~mL})$ ) followed by heating.

\section{NMR Spectroscopy}

${ }^{1} \mathrm{H}$ NMR data was acquired on a Bruker AVANCE400 (400 MHz), Bruker DRX-400 (400 MHz) or Bruker AVANCEIII-400 (400 MHz) spectrometer. Chemical shifts $(\delta)$ are reported in parts per million (ppm) relative to incompletely deuterated $\mathrm{CDCl}_{3}$ (s, $7.26 \mathrm{ppm}$ ), $\mathrm{C}_{6} \mathrm{D}_{6}\left(\mathrm{~s}, 7.16 \mathrm{ppm}\right.$ ), or $\mathrm{CD}_{3} \mathrm{CN}$ (quint, $1.94 \mathrm{ppm}$ ). Splitting patterns are designated as s, singlet; d, doublet; t, triplet; q, quartet; quint, quintet; hept, hepted; m, multiplet; br, broad. Proton-decoupled ${ }^{13} \mathrm{C}$ NMR data were acquired on a Bruker AVANCE400 (101 MHz), Bruker DRX-400 (101 MHz) or Bruker AVANCEIII-400 (101 MHz). Chemical shifts are reported in ppm relative to $\mathrm{CDCl}_{3}(77.16 \mathrm{ppm})$ or $\mathrm{C}_{6} \mathrm{D}_{6}$ (128.06 ppm). ${ }^{31} \mathrm{P}$ NMR data was acquired on a Bruker AVANCE400 (162 MHz) spectrometer. ${ }^{19} \mathrm{~F}$ NMR data was acquired on a Bruker AVANCE400 (376 MHz) spectrometer. Chemical shifts $(\delta)$ are reported in parts per million $(\mathrm{ppm})$ and were referenced using the $\Xi$-scales with $85 \% \mathrm{H}_{3} \mathrm{PO}_{4}\left(\Xi=40.480747 \mathrm{MHz},{ }^{31} \mathrm{P}\right)$ and $\mathrm{CCl}_{3} \mathrm{~F}$ $\left(\Xi=94.094011 \mathrm{MHz},{ }^{19} \mathrm{~F}\right)$ as secondary references.

\section{Cyclic Voltammetry}

Cyclic voltammetry data was acquired on a BioLogic SP-150 Potentiostat. $\mathrm{Bu}_{4} \mathrm{NPF}_{6}$ was recrystalized from EtOH and dried in vacuo prior use. All measurements were performed under nitrogen atmosphere inside a glovebox. Supporting electrolyte: $\mathrm{Bu}_{4} \mathrm{NPF}_{6}(0.1 \mathrm{M}$ in $\mathrm{MeCN})$, working electrode: glassy carbon disc $(\varnothing=0.3 \mathrm{~mm})$, counter

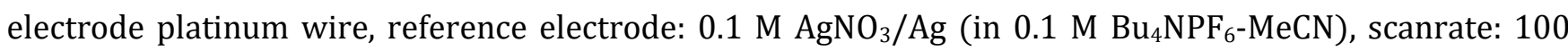
$\mathrm{mV} / \mathrm{s}$, analyte concentration: $10 \mathrm{mM}$. All spectra are referenced against the ferrocene/ferrocene ${ }^{+}$couple as an internal standard. 


\section{UV/VIS Spectroscopy}

UV/VIS data was acquired on an Agilent Cary 60 UV-Vis. All measurements were performed in an oven-dried, sealable quartz glass cuvette, which was charged inside and nitrogen-filled glovebox with the analytes and solvent. The concentration of the analytes was $\mathrm{c}=0.04 \mathrm{M}$ in $\mathrm{MeCN}$.

\section{Infrared Spectroscopy}

Infrared (IR) data was recorded on an Alpha-P Bruker FT-IR Spectrometer. Absorbance frequencies are reported in reciprocal centimeters $\left(\mathrm{cm}^{-1}\right)$. The intensity of the absorbance frequencies is indicated with: $(\mathrm{w})$, weak, $(\mathrm{m})$, medium; (s), strong and (br), broad.

\section{Melting Points}

Melting points (Mp) were measured on a Büchi Melting Point B-540 and are uncorrected.

\section{Mass Spectrometry}

HRMS ESI measurements were performed by an Agilent LC-MS TOF and XEVO G2-S QTOF mass spectrometers. APPI/LTQ-Orbitrap-FT-MS analyses were performed on a Thermo Orbitrap Elite and LTQ Orbitrap FTMS instrument. High resolution mass values are given in $\mathrm{m} / \mathrm{z}$. 


\section{Irradiation Setups}

a) The setup for the irradiation with white LEDs consisted of a photoreactor made out of a metal drum $(\mathrm{h}=26 \mathrm{~cm}$, $\varnothing=14 \mathrm{~cm}$ ), in which white LED stripes from Ledxon were attached. On top of the drum, a fan was installed. For irradiation of a vial, it was hold in a beaker on a stirring plate and the drum was placed over the stirring plate. The distance between the LEDs and the reaction vial was $6 \mathrm{~cm}$. The reaction chamber was cooled by the fan.

b) The setup for the irradiation with the Kessil PR-160 lamps (427 and $390 \mathrm{~nm}$ ), consisted of the specific lamp, a stirring plate, a clamp to hold the vial in place and a fan. To ensure efficient cooling two tubes, connected to a compressed air outlet, were directly pointed to the reaction vial. The distance between the Kessil lamps and the reaction vial was $2 \mathrm{~cm}$. For all irradiation setups, thermo-coupled experiments showed a constant reaction temperature of $26^{\circ} \mathrm{C}$.
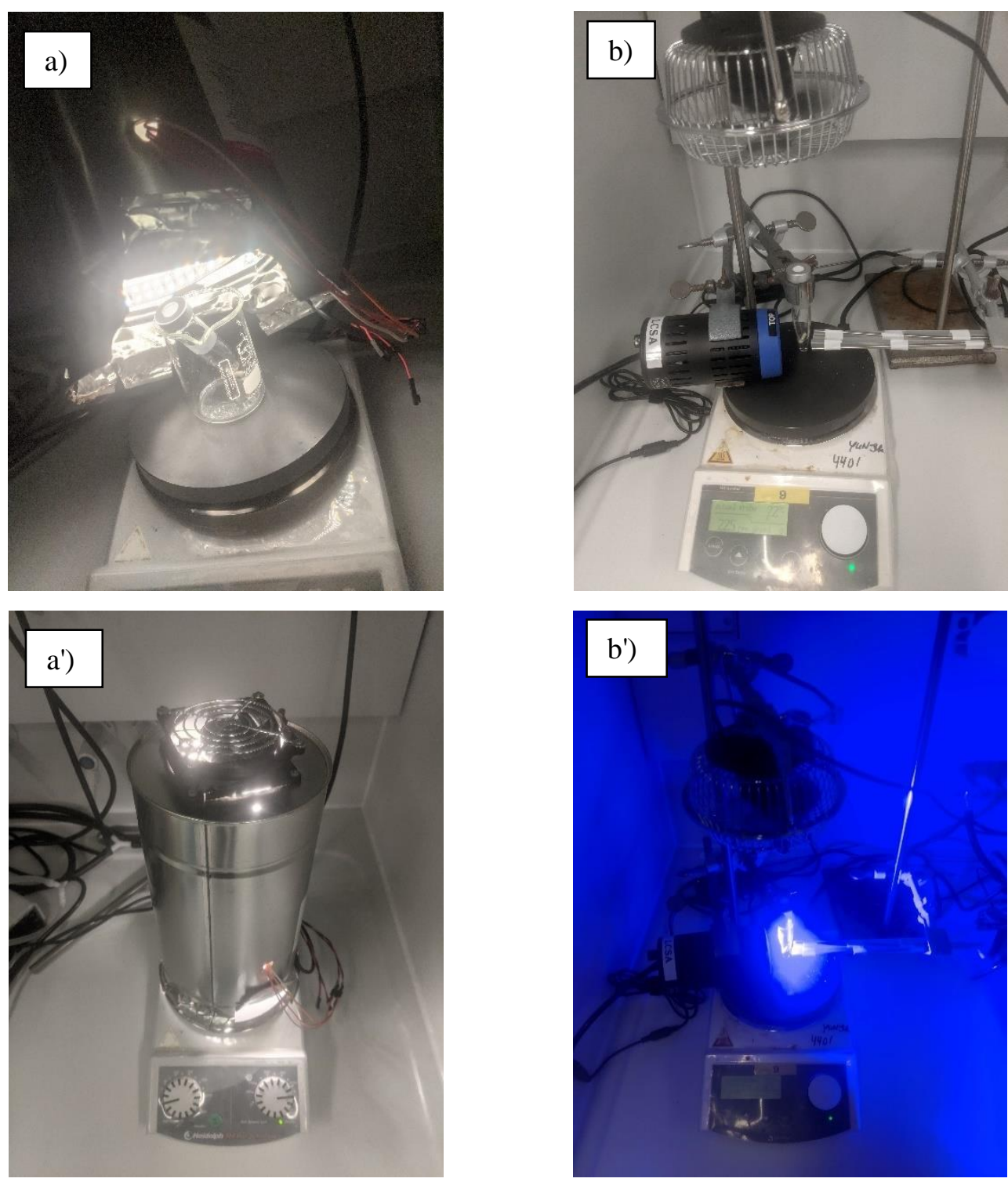

Figure S1: Setup for the irradiation with white LEDs: a) view into the photoreactor, a') closed reactor. Setup for the irradiation with Kessil lamps (390 nm and $427 \mathrm{~nm}$ ): without b) and with irradiation b'). 


\subsection{Condition Screening}

\section{General Procedure A:}

A $1 \mathrm{~mL}$ microwave vial, equipped with a stir bar was charged with $10 \mu \mathrm{mol}$ substrate and transferred into the glovebox. MeCN was added, followed by the additive, DAP and $110 \mu \mathrm{mol}$ HBpin. The vial was capped, removed from the glovebox and irradiated for $16 \mathrm{~h}$ at rt. Volatiles were removed under reduced pressure and the residue was directly subjected to ${ }^{1} \mathrm{H}$ NMR analysis.

Table S1: Condition Screening for DAP catalyzed reductive Cyclizations of 1-3a ${ }^{[a]}$

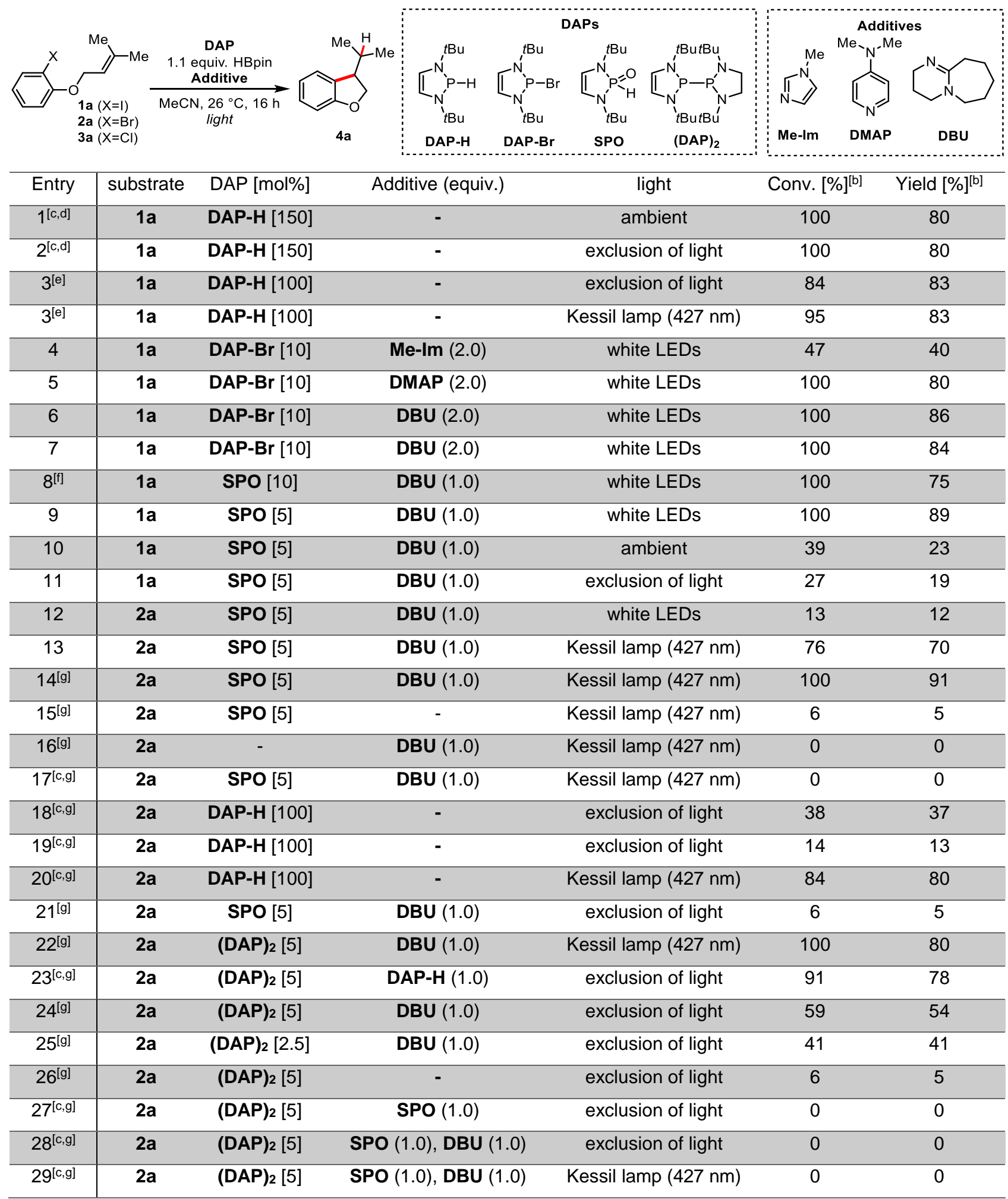


[a] GP A; conditions: $100 \mu \mathrm{mol}$ 1a-3a, 2.5-150 $\mu \mathrm{mol} \boldsymbol{D A P}, 110 \mu \mathrm{mol} \mathrm{HBpin,} \mathrm{100-200} \mu \mathrm{mol}$ additive in $\mathrm{MeCN}(0.1 \mathrm{M})$ at $26^{\circ} \mathrm{C}$ for $16 \mathrm{~h}$.

[b] Determined by ${ }^{1} \mathrm{H}-\mathrm{NMR}$ using 1,3,5-trimethoxybenzene as an internal standard. [c] no HBpin. [d] $2 \mathrm{~h}$ reaction time. [e] $4 \mathrm{~h}$ reaction time.

[f] 0.33 equiv. $\mathrm{PhSiH}_{3}$ instead of $\mathrm{HB}$ in. [g] $\mathrm{c}$ (substrate)=0.8 $\mathrm{M}$ in $\mathrm{MeCN}$.

\subsection{Mechanistic Investigations}

\subsubsection{Cyclic Voltammetry}

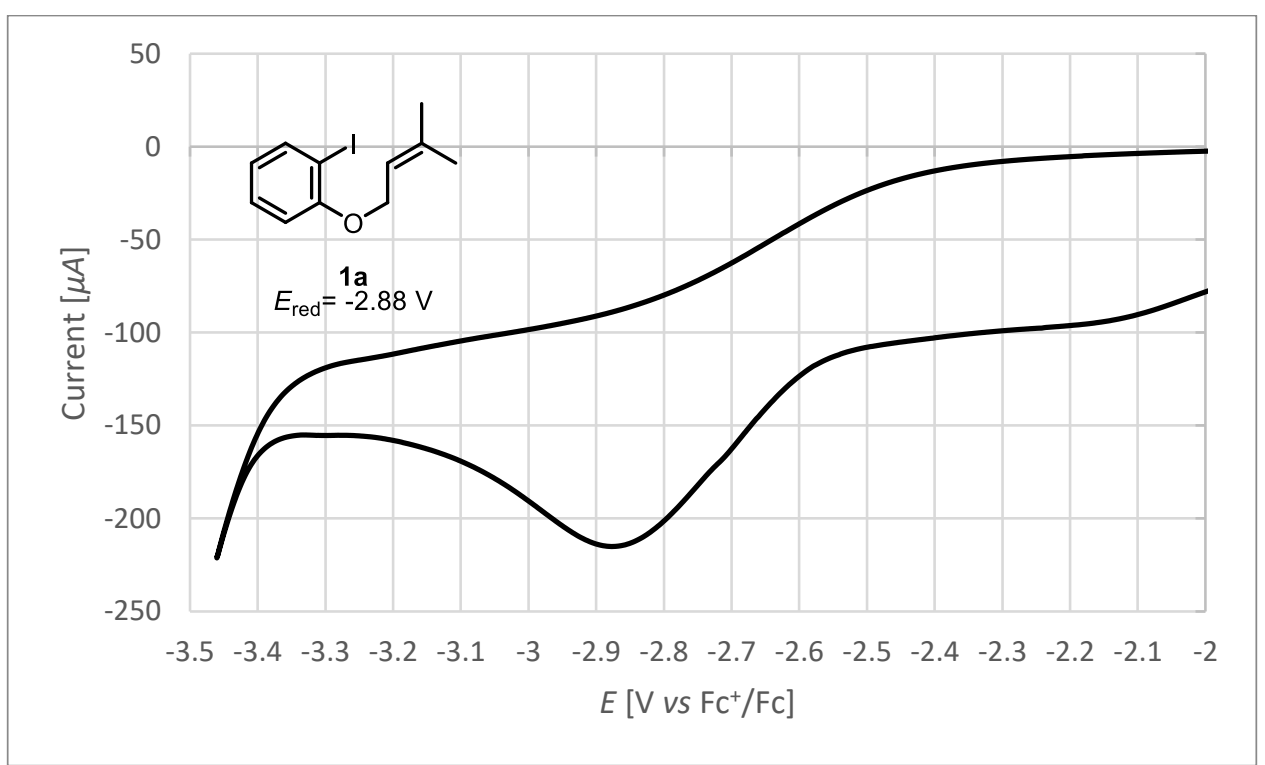

Figure S2: Cyclic voltammetry of substrate 1a. The irreversible reduction peak potential $E_{\text {red }}$ was determined to be at $-2.88 \mathrm{~V}$.

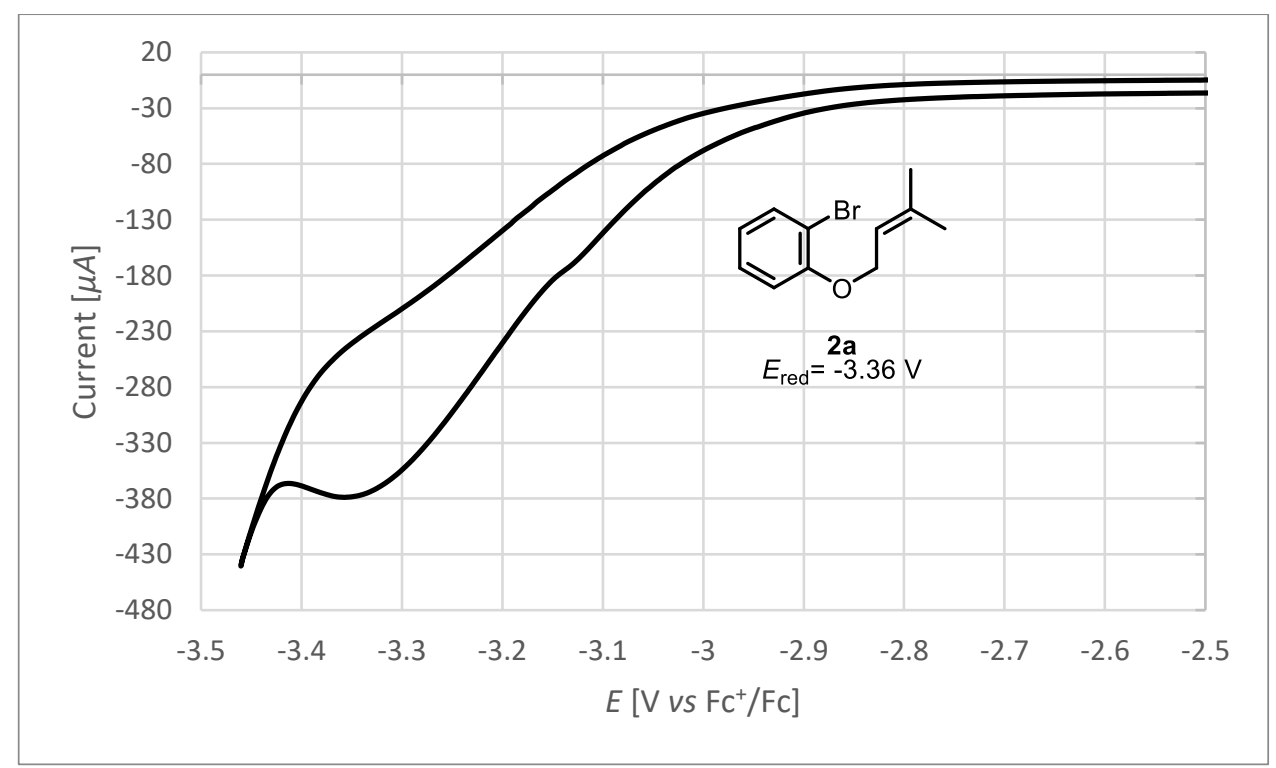

Figure S3: Cyclic voltammetry of substrate 2a. The irreversible reduction peak potential $E_{\text {red }}$ was determined to be at $-3.36 \mathrm{~V}$. 


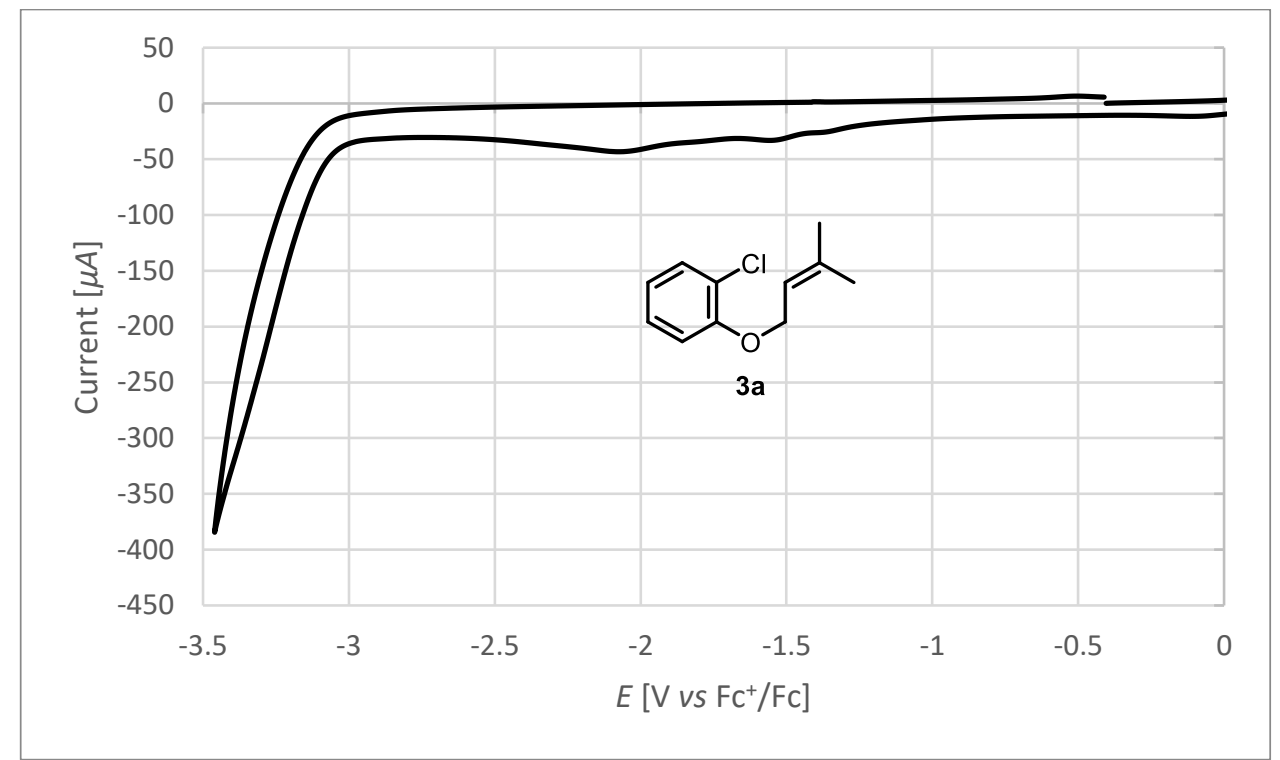

Figure S4: Cyclic voltammetry of substrate 3a. Its reduction peak was found to be outside the solvent window.

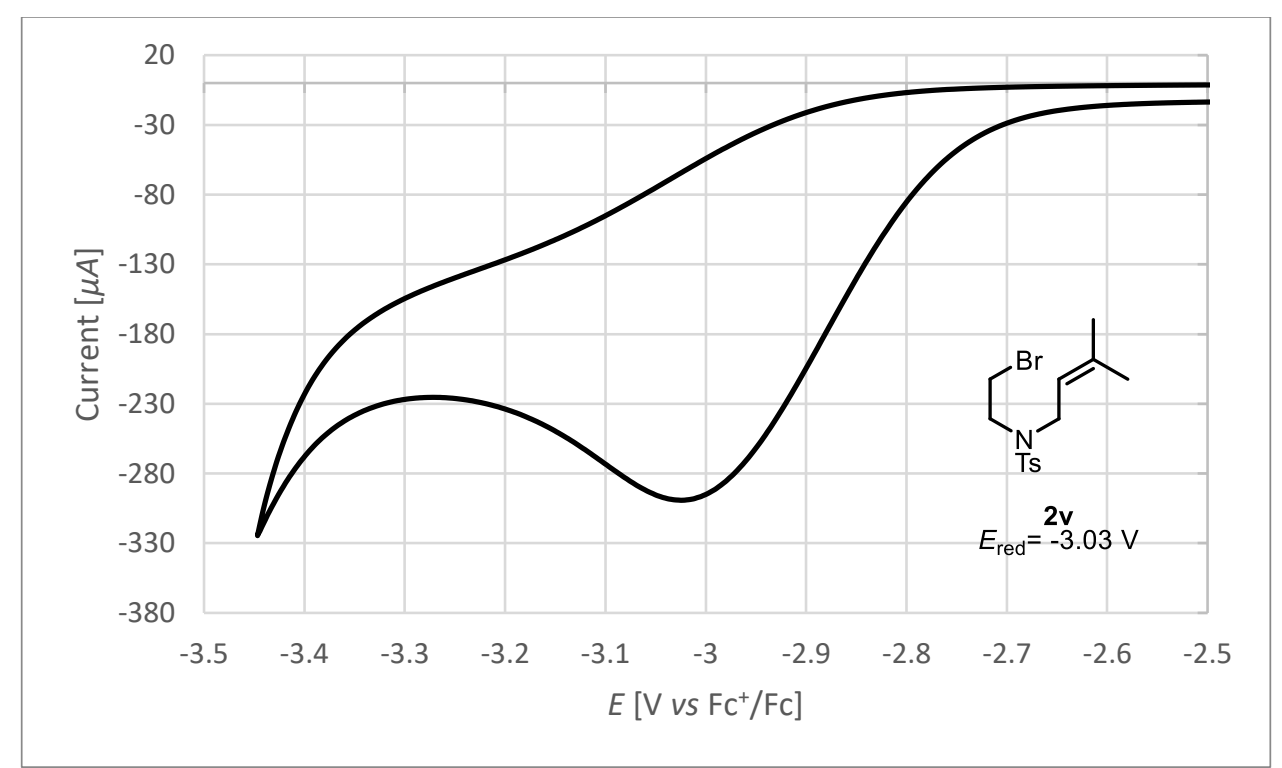

Figure S5: Cyclic voltammetry of substrate $2 \mathbf{v}$. The irreversible reduction peak potential $E_{\text {red }}$ was determined to be at $-3.03 \mathrm{~V}$. 


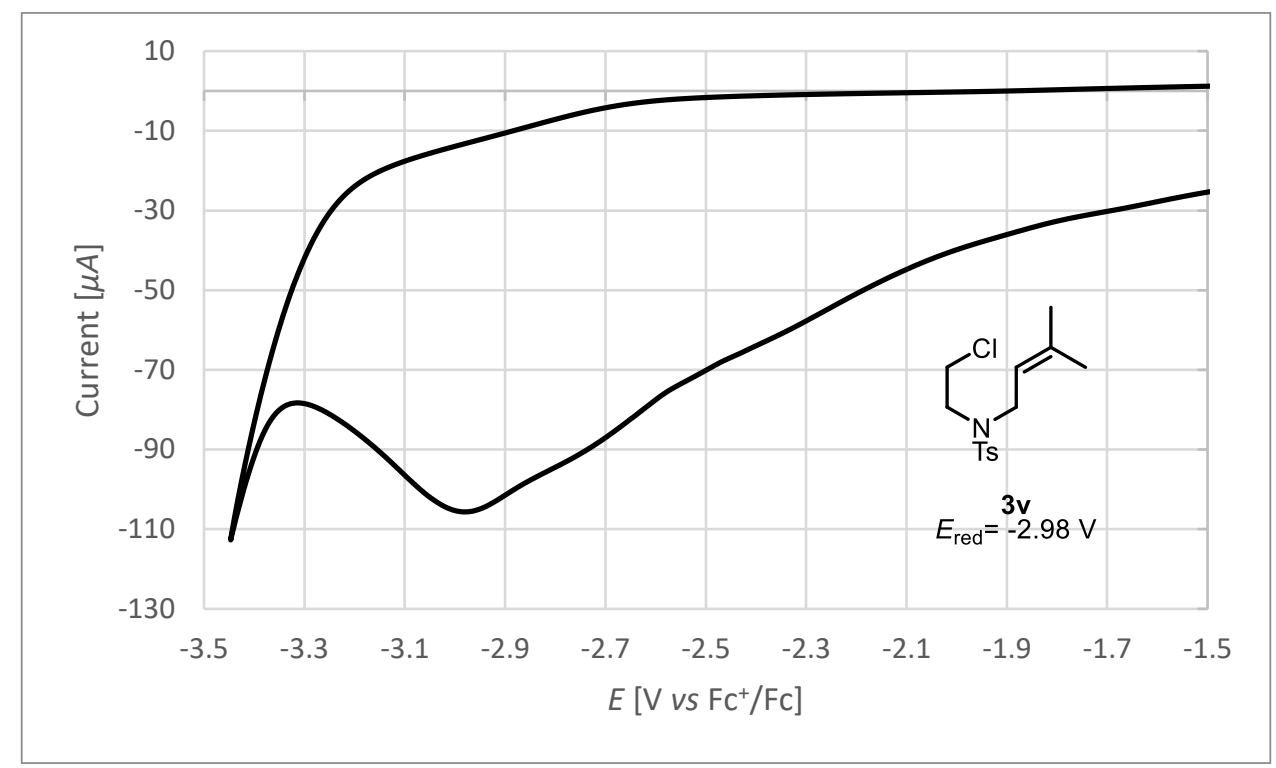

Figure S6: Cyclic voltammetry of substrate 3v. The irreversible reduction peak potential $E_{\mathrm{p}}{ }^{\text {red }}$ was determined to be at $-2.98 \mathrm{~V}$.

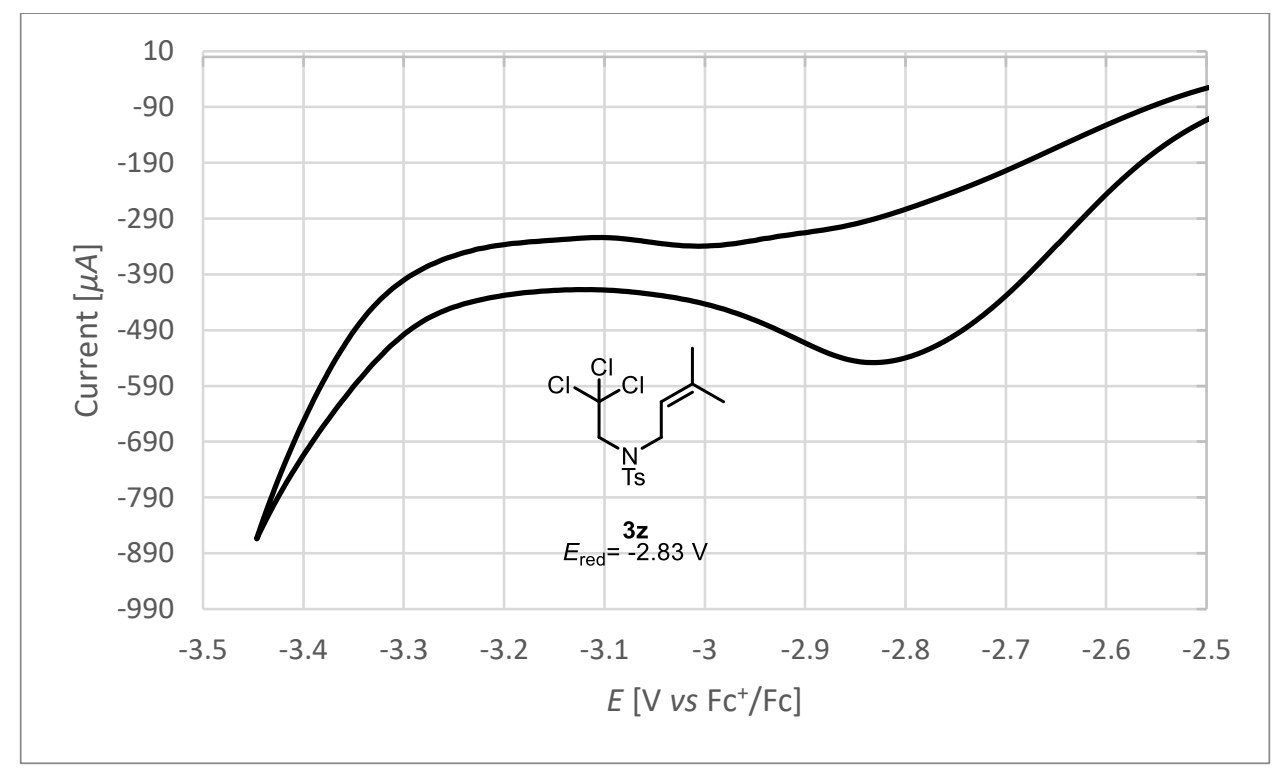

Figure S7: Cyclic voltammetry of substrate $3 z$. The irreversible reduction peak potential $E_{\text {red }}$ was determined to be at $-2.83 \mathrm{~V}$. 


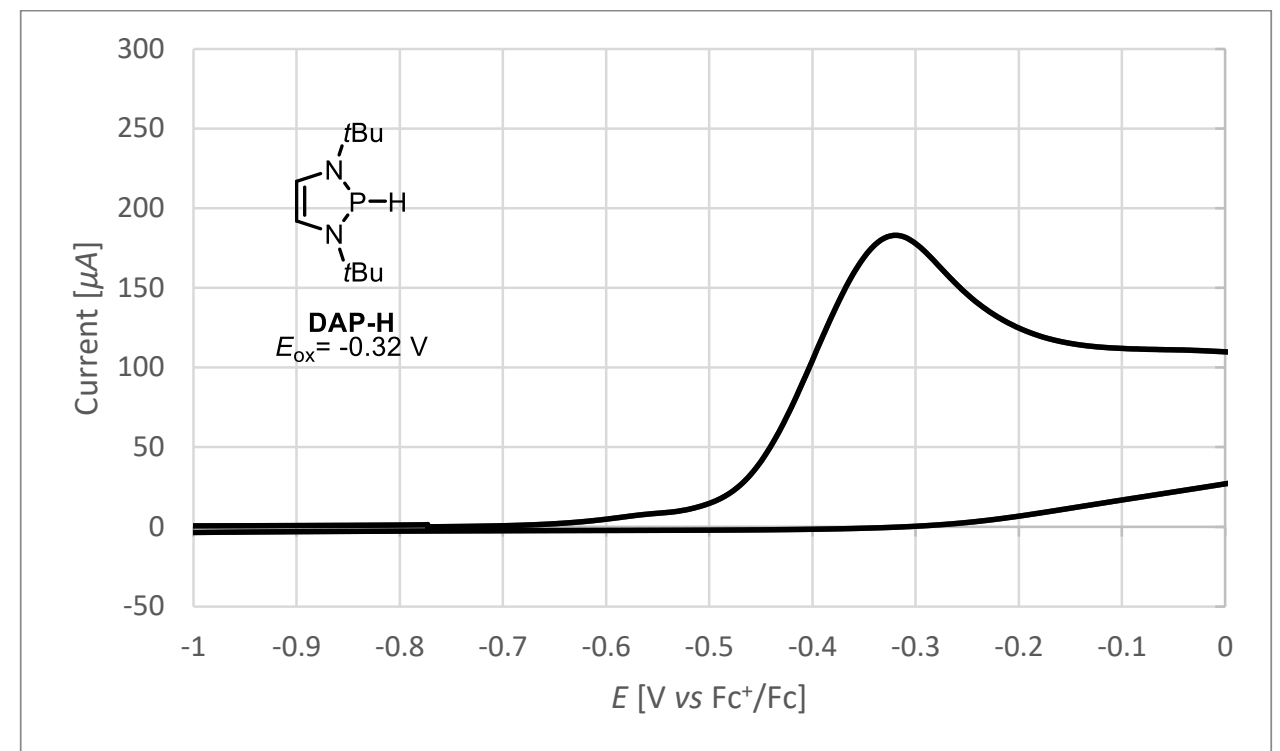

Figure S8: Cyclic voltammetry of DAP-H. The irreversible oxidation peak potential $E_{\mathrm{ox}}$ was determined to be at $-0.32 \mathrm{~V}$.

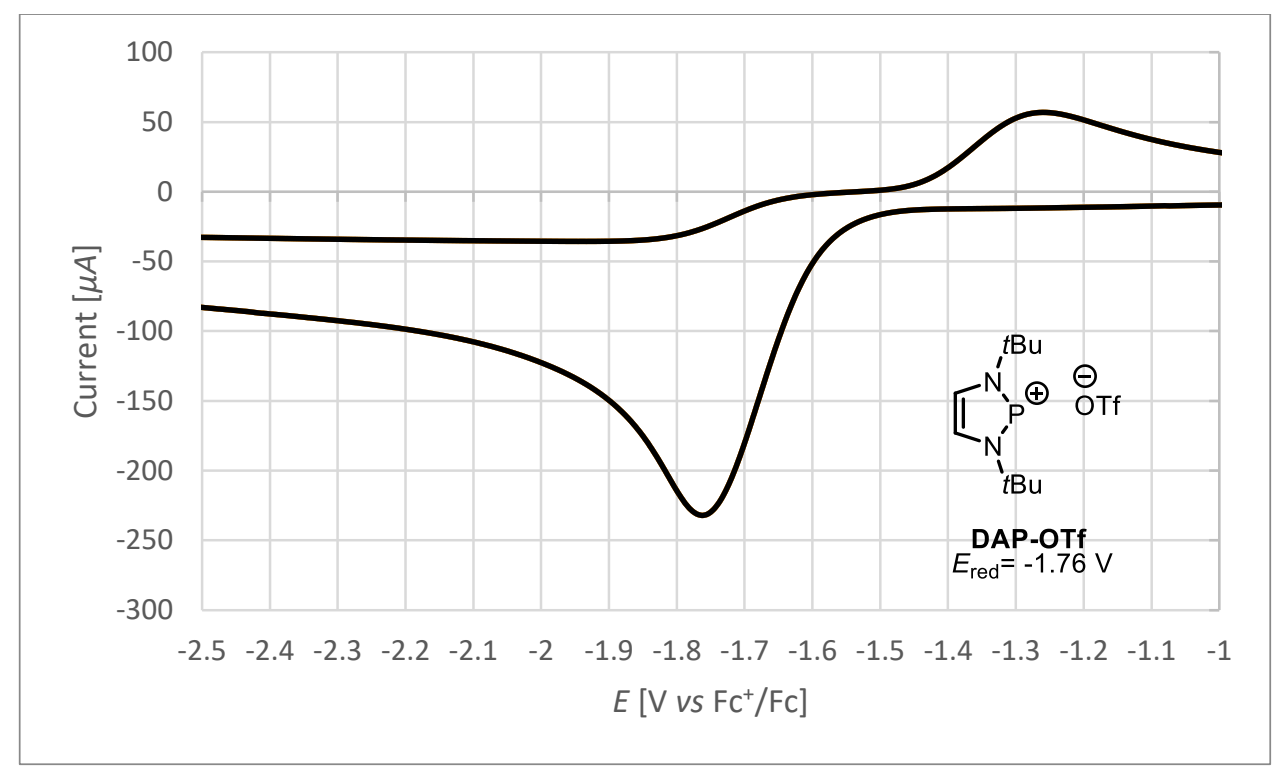

Figure S9: Cyclic voltammetry of DAP-OTf. The irreversible reduction peak potential $E_{\text {red }}$ was determined to be at $-1.76 \mathrm{~V}$. 


\subsubsection{UV/VIS measurements}

UV/Vis spectra of 1a and DAP-I

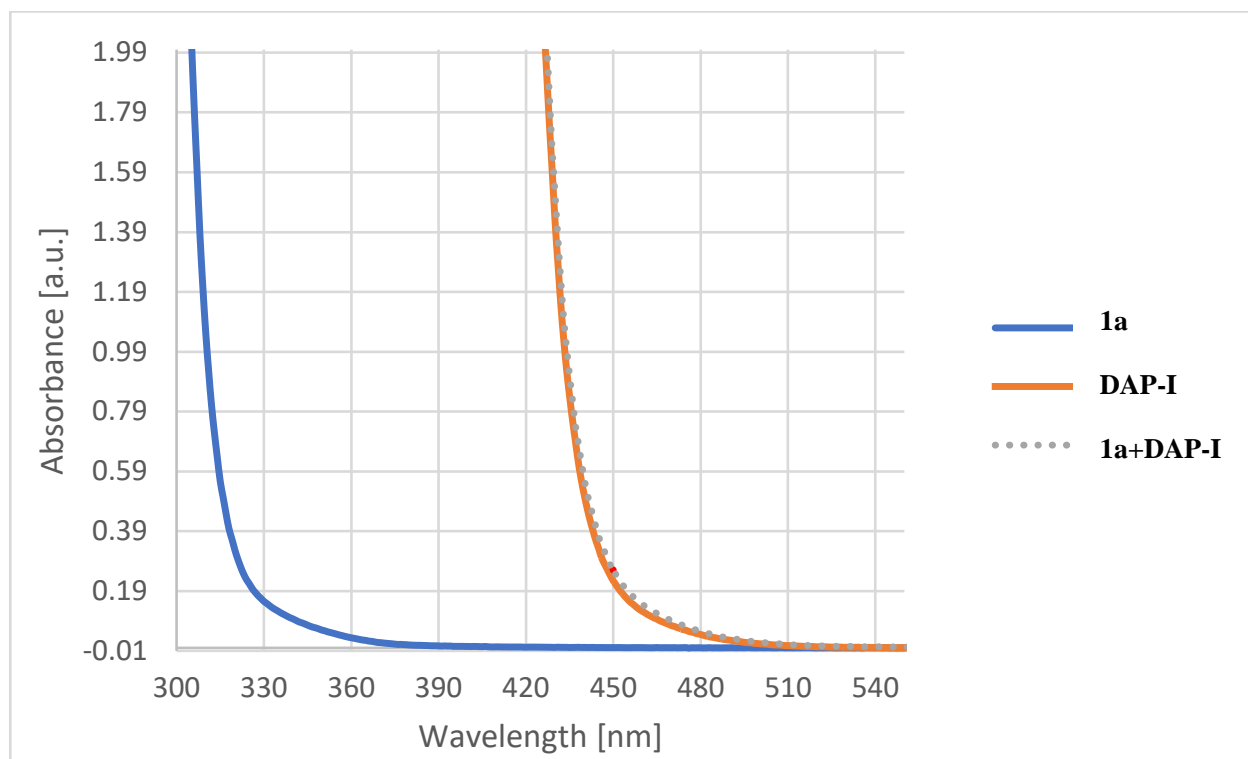

Figure S10: UV-Vis absorption spectra of 1a (blue, full line), DAP-I (orange, dashed line) and a mixture of 1a and DAP-I (grey, dotted line). Analyte concentration: 0.04 M in MeCN. No formation of an EDA complex was observed.

UV/Vis spectra of 1a and DAP-H

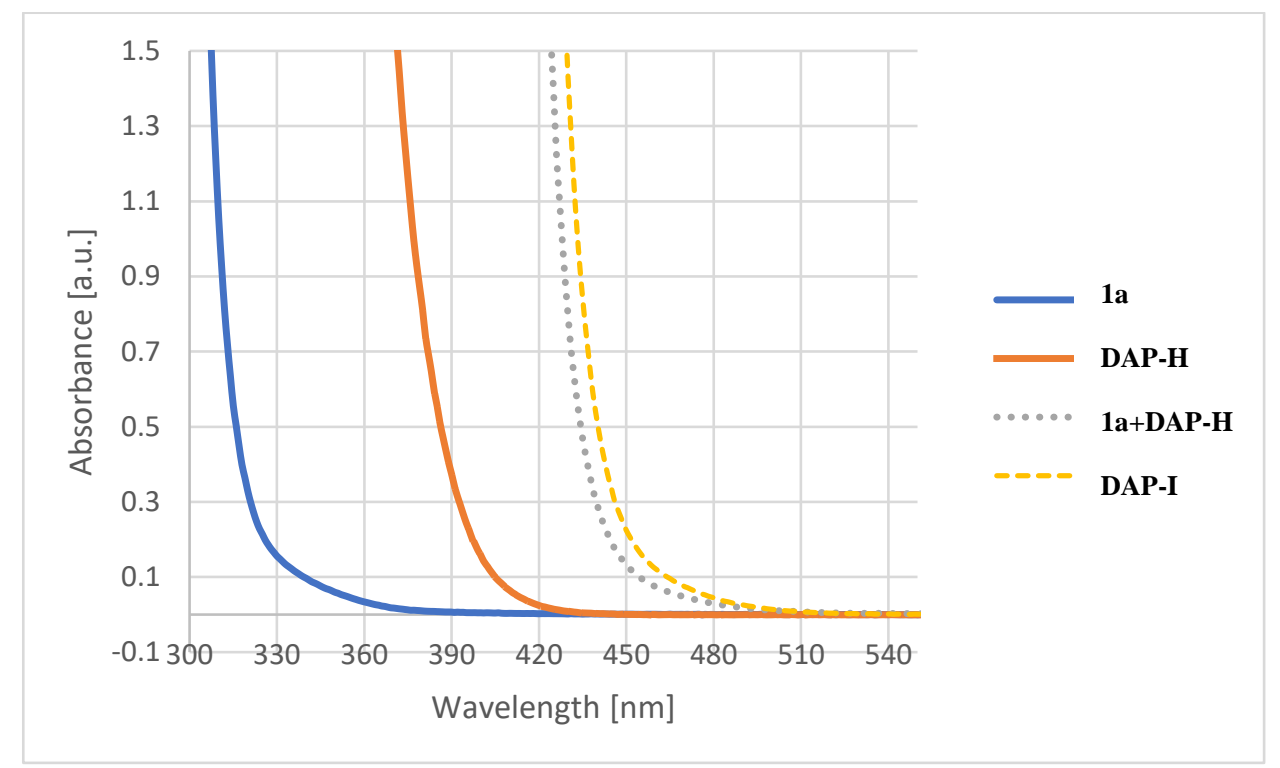

Figure S11: UV-Vis absorption spectra of 1a (blue, full line), DAP-H (orange, dashed line), a mixture of 1a and DAP-H (grey, dotted line) and DAP-I (yellow, long dashed line). Analyte concentration: 0.04 M in MeCN. Rapid reaction of 1a and DAP-H resulting in formation of DAP-I was observed. 
UV/Vis spectra of $\mathbf{2 a}$ and DAP-Br

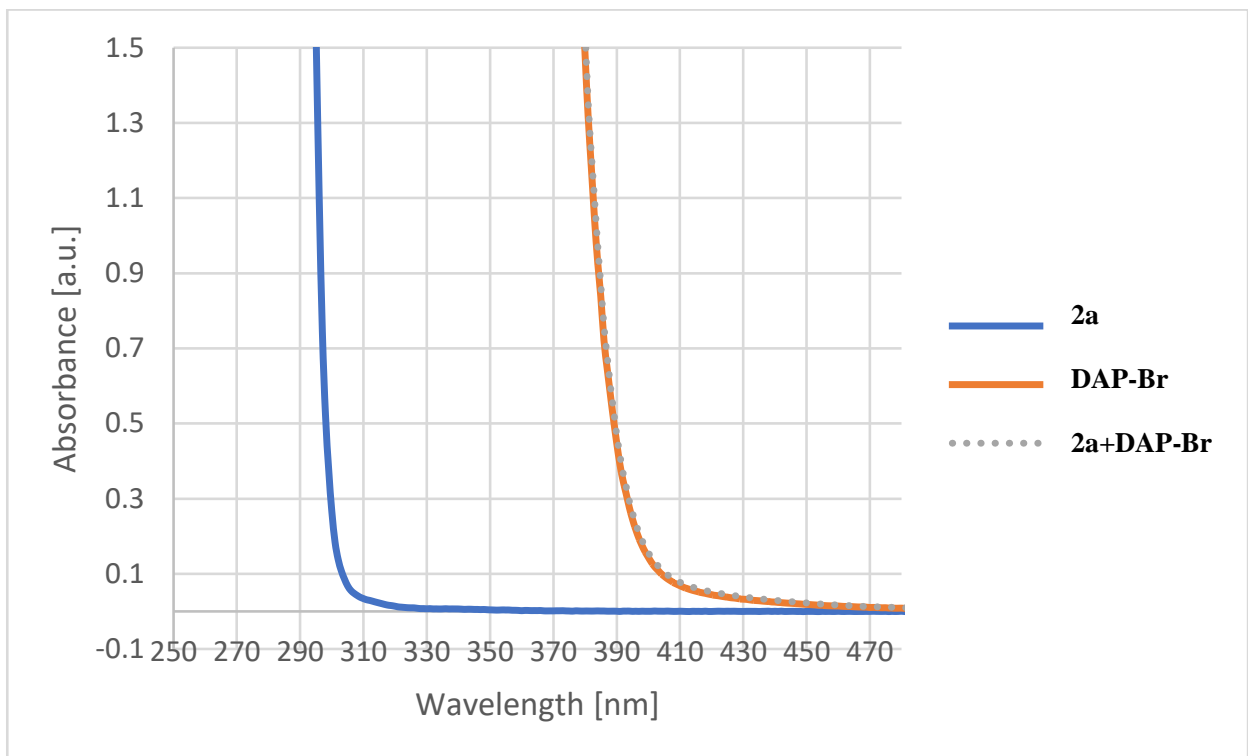

Figure S12: UV-Vis absorption spectra of 2a (blue, full line), DAP-Br (orange, dashed line) and a mixture of 2a and DAP-Br (grey, dotted line). Analyte concentration: $0.04 \mathrm{M}$ in $\mathrm{MeCN}$. No formation of an EDA complex was observed.

\section{UV/Vis spectra of 2a and DAP-H}

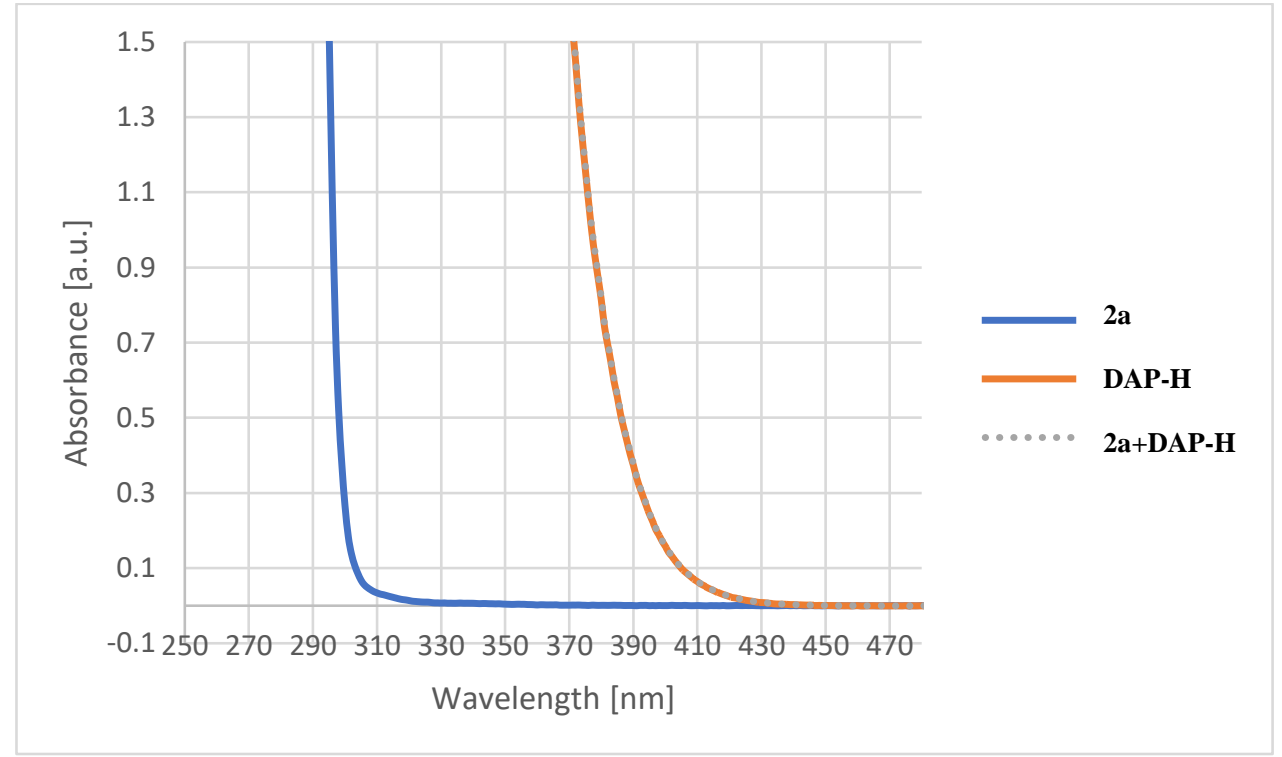

Figure S13: UV-Vis absorption spectra of 2a (blue, full line), DAP-H (orange, dashed line) and a mixture of 2a and DAP-H (grey, dotted line). Analyte concentration: 0.04 M in MeCN. No formation of an EDA complex was observed. 


\section{Estimation of Excited State Oxidation Potential of DAP-H.}

The estimated excited state oxidation potential $E^{0}\left(\mathbf{D A P}-\mathbf{H}^{+} / \mathbf{D A P}-\mathbf{H}^{*}\right)$ was calculated according to equation (1) ${ }^{[1,2]}$ :

$$
E^{0}\left(\mathbf{D A P}-\mathbf{H}^{+} / \mathbf{D A P}-\mathbf{H}^{*}\right)=E^{0}\left(\mathbf{D A P}-\mathbf{H}^{+} / \mathbf{D A P}-\mathbf{H}\right)-E_{00}\left(\mathbf{D A P}-\mathbf{H}^{*} / \mathbf{D A P}-\mathbf{H}\right)
$$

Since the electrochemical oxidation of DAP-H was irreversible (see Figure S8), the irreversible peak potential $E_{\mathrm{ox}}$ was used for $E^{0}\left(\mathbf{D A P}-\mathbf{H}^{+} / \mathbf{D A P}-\mathbf{H}\right) . E_{00}\left(\mathbf{D A P}-\mathbf{H}^{*} / \mathbf{D A P}-\mathbf{H}\right)$, which is the excited state energy of the DAP-H, was estimated spectroscopically from the position of the long wavelength tail of the absorption spectrum (see Figure S14) recorded in $\mathrm{MeCN}$, the same solvent used for the electrochemical analysis.

For DAP-H, $E_{\text {ox }}$ was measured to be $-0.32 \mathrm{~V}$, which provides $E^{0}\left(\mathbf{D A P}-\mathbf{H}^{+} / \mathbf{D A P}-\mathbf{H}\right)=-0.32 \mathrm{~V}$. The position of the long wavelength tail of the absorption spectrum corresponds to $440 \mathrm{~nm}$, which translates into an $E_{00}\left(\mathbf{D A P}-\mathbf{H}^{*} / \mathbf{D A P}-\mathbf{H}\right)$ of $2.82 \mathrm{eV}$.

$$
E^{0}\left(\mathbf{D A P}-\mathbf{H}^{+} / \mathbf{D A P}-\mathbf{H}^{*}\right)=-0.32 \mathrm{~V}-2.82 \mathrm{~V}=-3.14 \mathrm{~V}
$$

The estimated excited state oxidation potential $E^{0}\left(\mathbf{D A P}-\mathbf{H}^{+} / \mathbf{D A P}-\mathbf{H}^{*}\right)=-3.14 \mathrm{~V}$ shows the SET from DAP-H* to substrate $1 \mathbf{a}\left(E_{\mathrm{red}}=-2.88 \mathrm{~V}\right)$ to be exergonic and for $\mathbf{2 b}\left(E_{\mathrm{red}}=-3.36 \mathrm{~V}\right)$ to be endergonic.

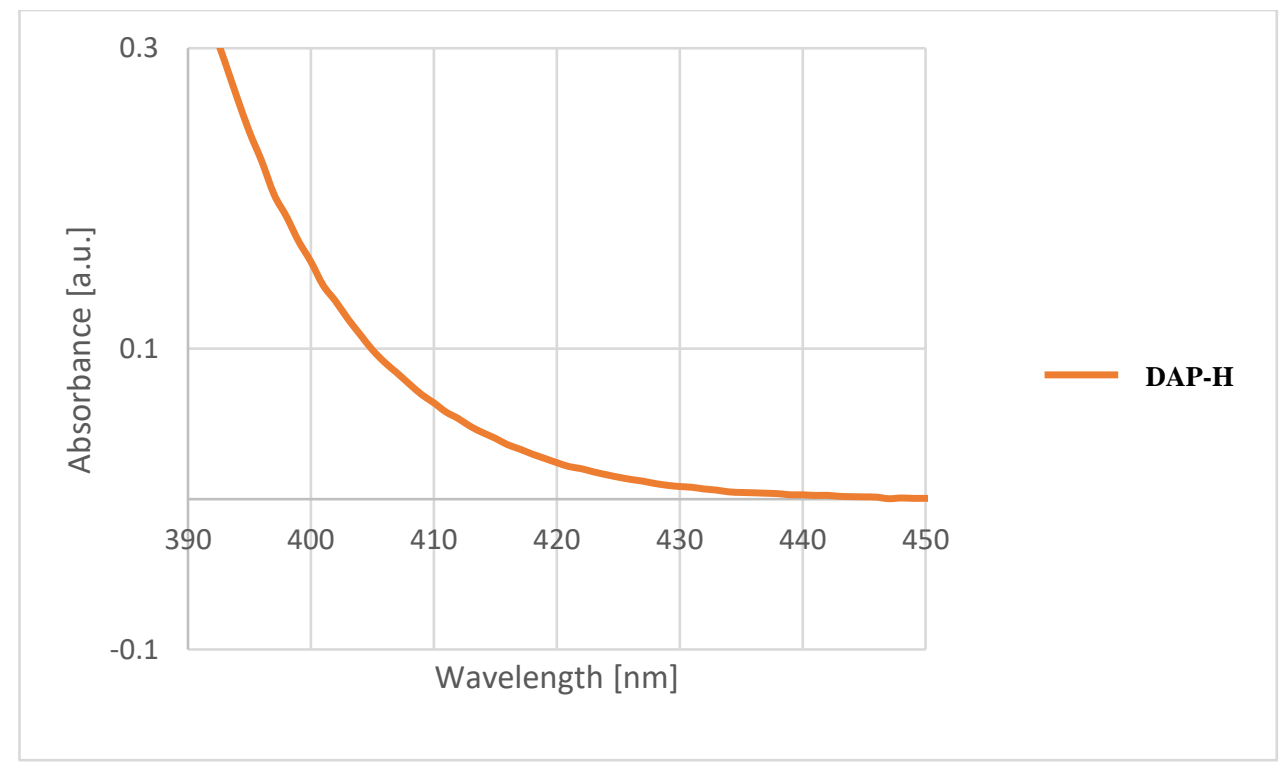

Figure S14: Zoom of the UV/Vis absorption spectra of DAP-H depicted in Figure S13. Analyte concentration: 0.04 M in MeCN. The long wavelength tail of the absorption of DAP-H was determined to be at $440 \mathrm{~nm}$. 


\subsubsection{Formation of DAP-H from DAP-I with DBU as an Activator}

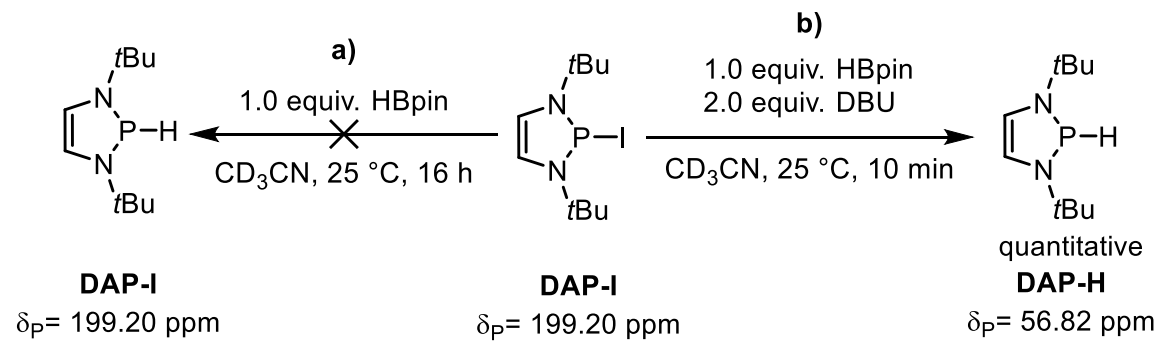

A dried J-Young NMR tube was charged with a solution of DAP-I (8.00 mg, $24.5 \mu \mathrm{mol}, 1.00$ equiv.) and HBpin (3.56 $\mu \mathrm{L}, 3.14 \mathrm{mg}, 24.5 \mu \mathrm{mol}, 1.00$ equiv.) in $\mathrm{CD}_{3} \mathrm{CN}(0.7 \mathrm{~mL})$ inside the glovebox. The tube was sealed and submitted to NMR analysis. No reaction towards DAP-H was observed, even after $16 \mathrm{~h}$. DAP-I was the only detectable species in ${ }^{31} \mathrm{P}$ NMR at $\delta=199.20 \mathrm{ppm}$ (Figure S15 a).

A dried J-Young NMR tube was charged with a solution of DAP-I (16.3 mg, $50.0 \mu \mathrm{mol}, 1.00$ equiv.), DBU (14.9 $\mu \mathrm{L}, 15.2 \mathrm{mg}, 100 \mu \mathrm{mol}, 2.00$ equiv.) and $\mathrm{HB}$ in $(7.25 \mu \mathrm{L}, 6.40 \mathrm{mg}, 50.0 \mu \mathrm{mol}, 1.00$ equiv. $)$ in $\mathrm{CD}_{3} \mathrm{CN}(1.0 \mathrm{~mL})$ inside the glovebox. The tube was sealed and submitted to NMR analysis. In ${ }^{31} \mathrm{P}$ NMR, rapid conversion of DAP-I to DAP-H as the only detectable species at $\delta=56.79$ ppm was observed (Figure S15 b).

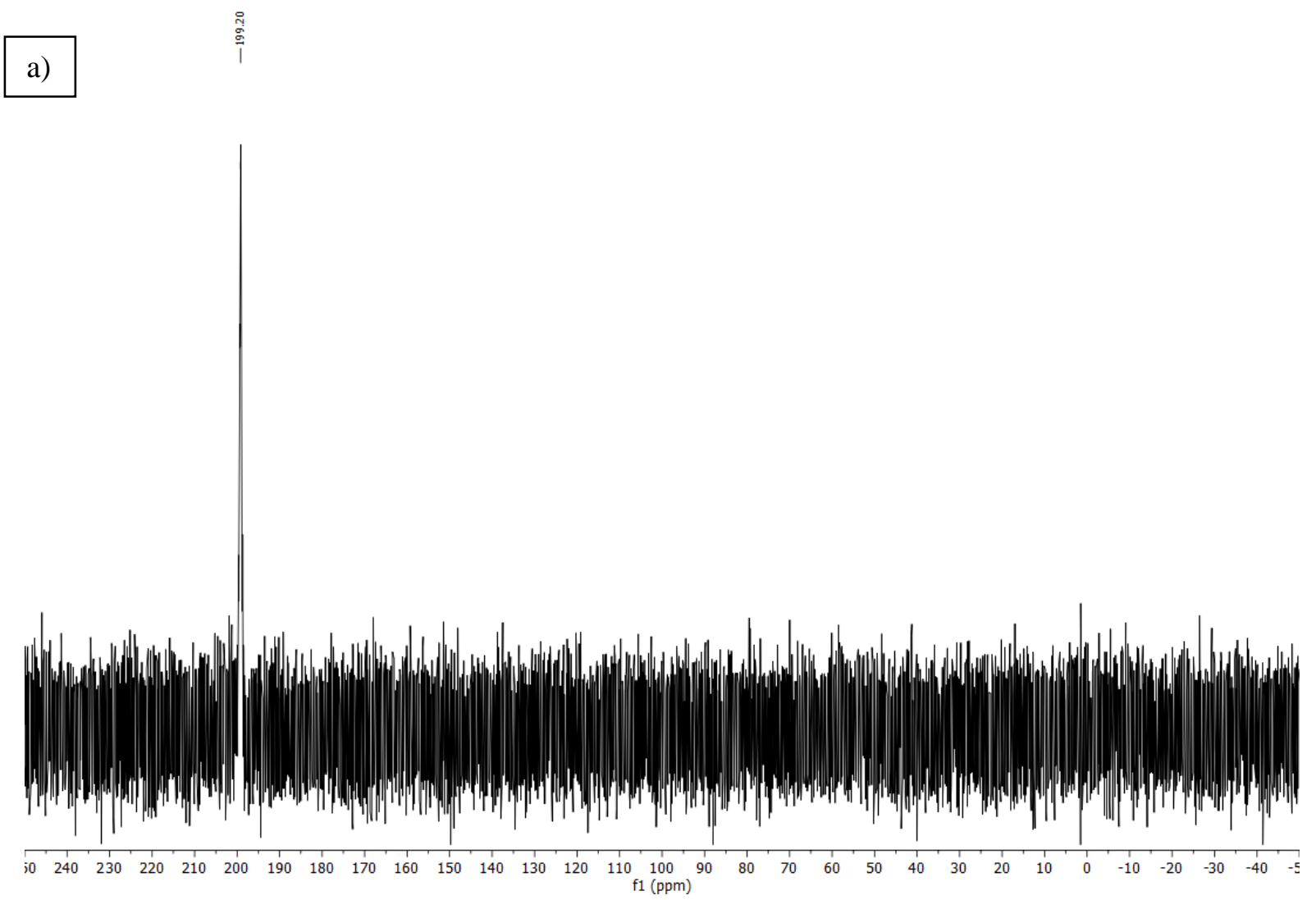




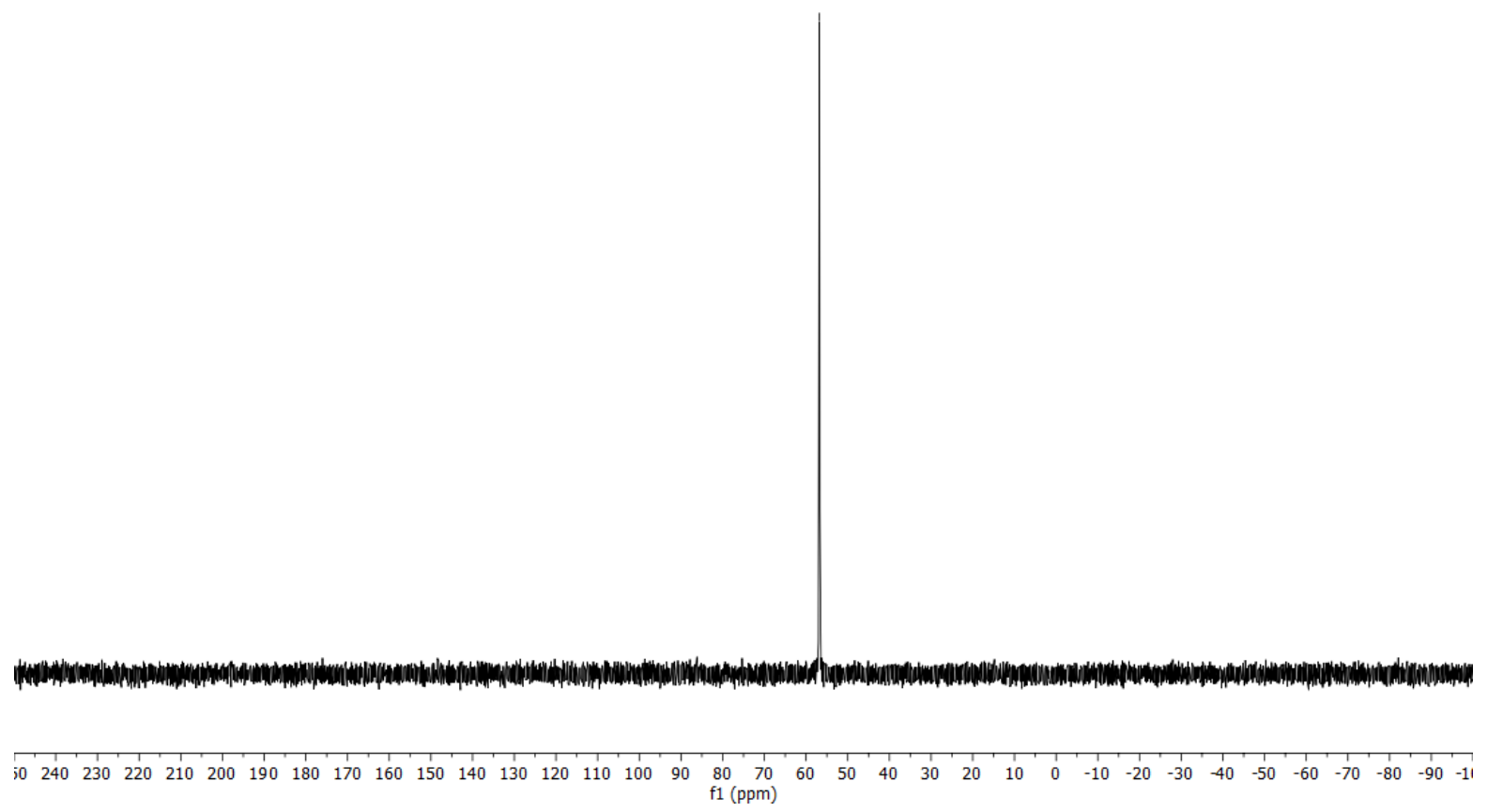

Figure S15: ${ }^{31}$ P NMR spectra of: a) a mixture of DAP-I (1.00 equiv.) and HBpin (1.00 equiv.) in $\mathrm{CD}_{3} \mathrm{CN}$ and b) DAP-I (1.00 equiv.), DBU (2.00 equiv.) and $\mathrm{HBpin}\left(1.00\right.$ equiv.) in $\mathrm{CD}_{3} \mathrm{CN}$. 


\subsubsection{Kinetic Investigations}

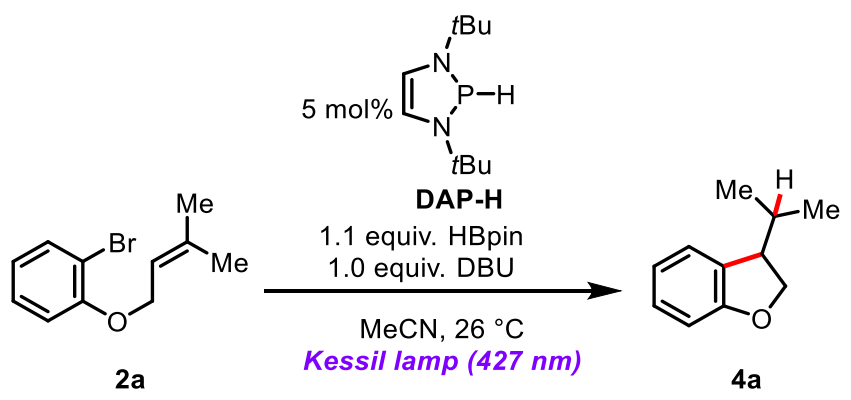

\section{General procedure B}

A flame dried $10 \mathrm{ml}$ microwave vial was charged with substrate $2 \mathrm{a}$ (120.6 mg, $0.5 \mathrm{mmol}, 1$ equiv.) and 1,3,5-trimethoxybenzene NMR standard (28.0 mg, $0.166 \mathrm{mmol}, 0.33$ equiv.) under air and the vial was introduced inside the glovebox. Next, dry and degassed MeCN (0.625 ml), DBU (76.1 mg, $75.0 \mu \mathrm{L}, 0.50 \mathrm{mmol}, 1.00$ equiv.), HBpin (70.4 mg, $80.0 \mu \mathrm{L}, 0.55 \mathrm{mmol}, 1.10$ equiv.) and DAP-H (5.0 mg, $0.025 \mathrm{mmol}, 0.05$ equiv.) were added. The tube was sealed and stirred under the irradiation of a Kessil lamp (427 nm). Samples of $50 \mu \mathrm{L}$ of this mixture were taken under a nitrogen atmosphere at specific times. The solvent was evaporated and the samples were analyzed by ${ }^{1} \mathrm{H}$ NMR.

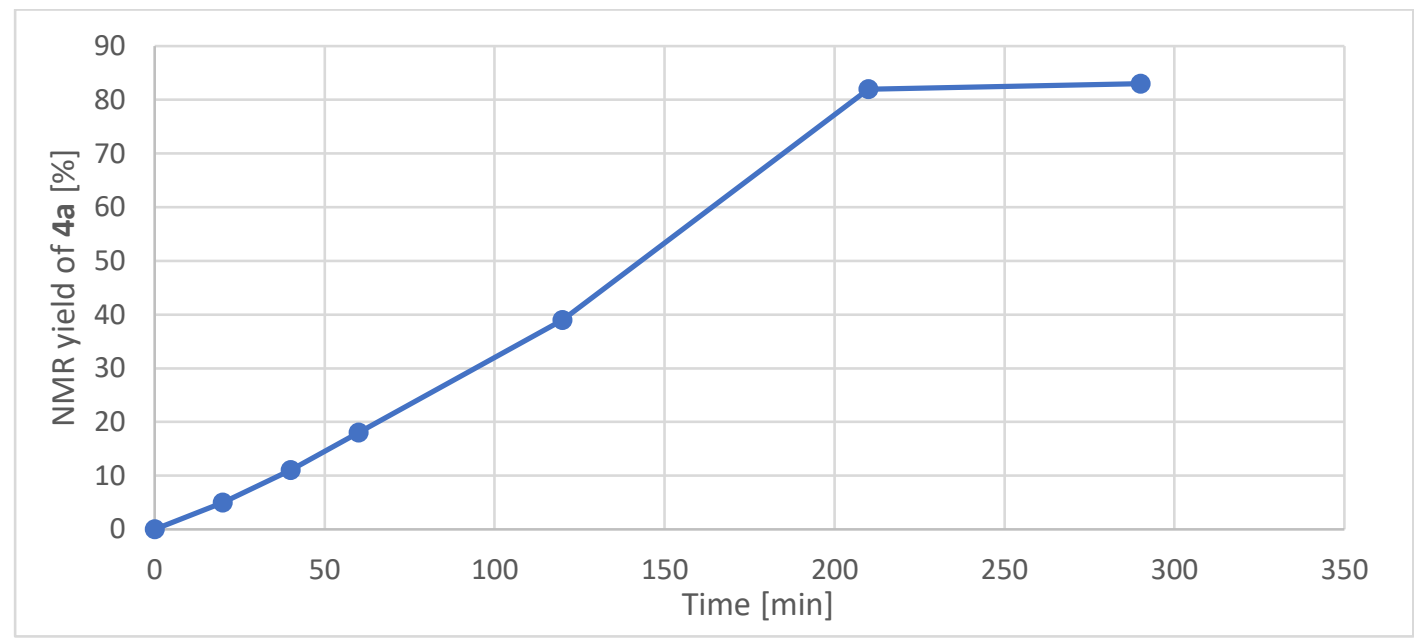

Figure S16: Reaction profile of DAP catalyzed cyclization of 2a.

The reaction shows a linear kinetic behavior at the initial stage of the reaction and no substantial induction period is observed. 


\section{$\underline{\text { Order dependence in catalyst DAP-H }}$}

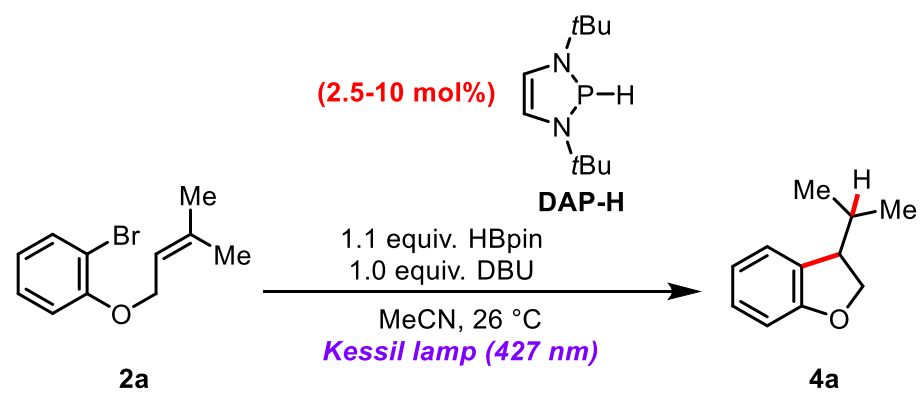

GP B was used with the following modifications: The amount of DAP-H (2.5 - $10 \mathrm{mg}, 0.0125-0.010 \mathrm{mmol}, 0.025$ - 0.100 equiv.) was varied.

a)

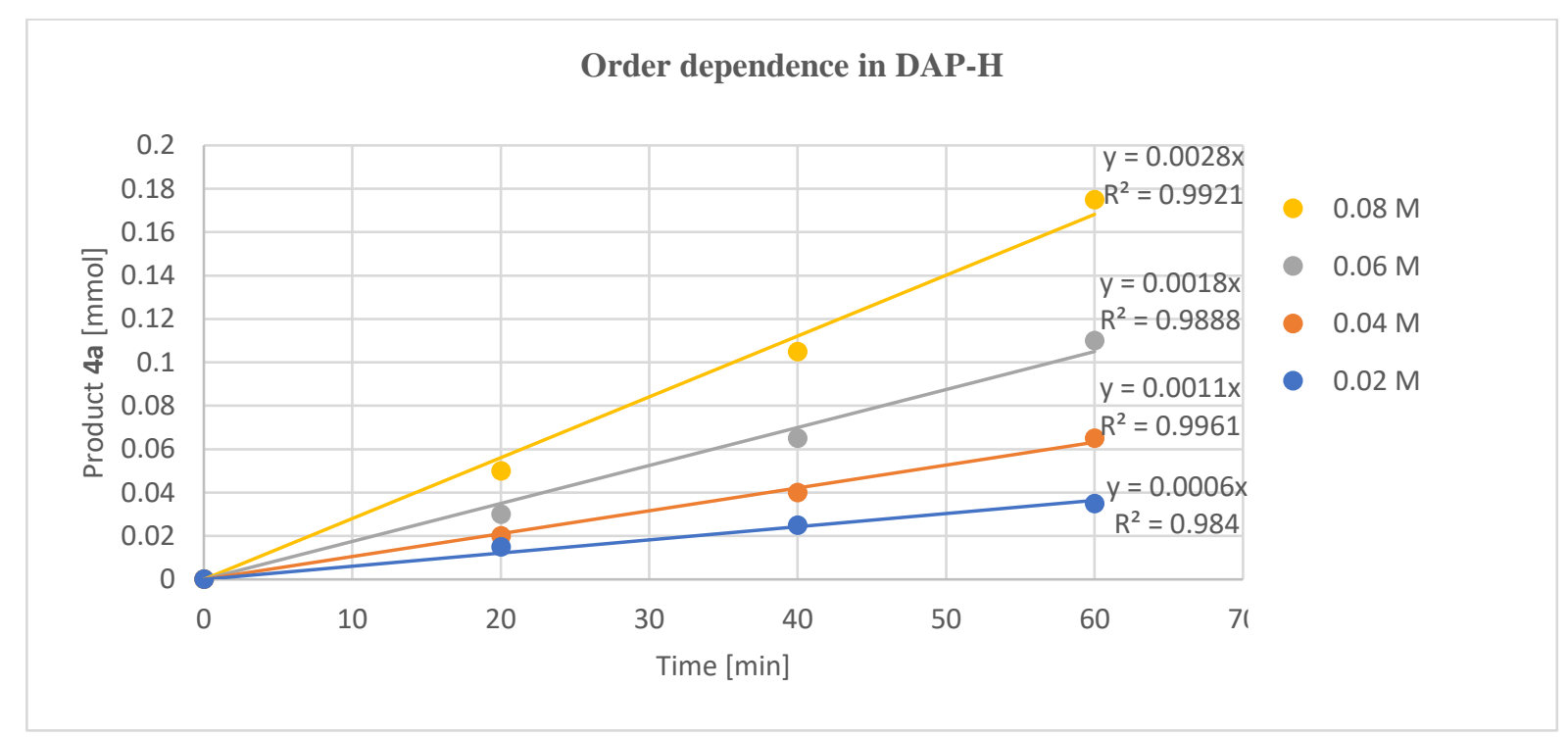

b)

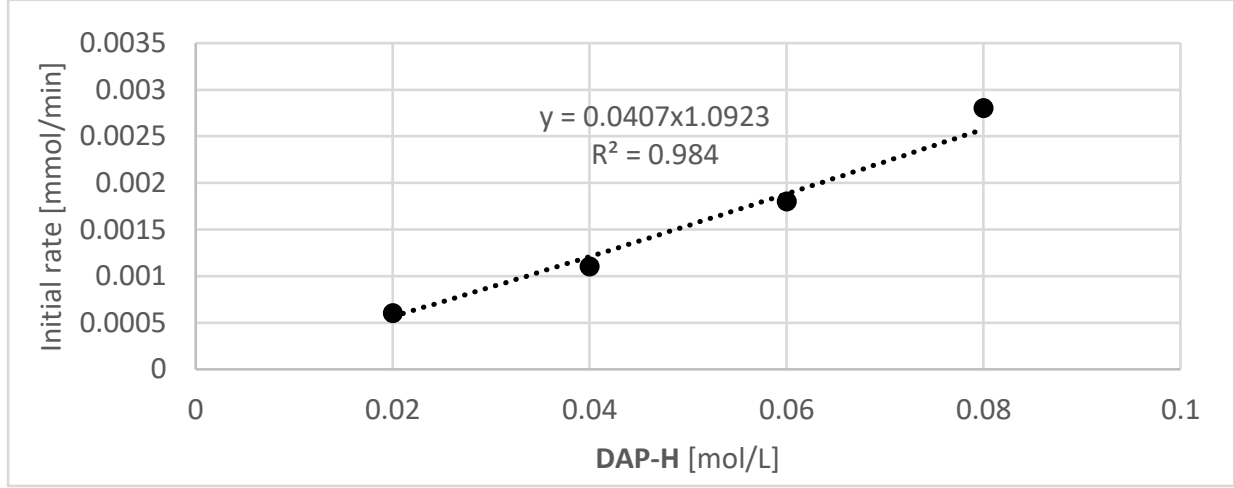

Figure S17: a) Reaction profiles at different initial concentrations of DAP-H showing a first-order dependence in DAP-H. Rate constants calculated from the slope of the plots. $[2 \mathrm{a}]=0.8 \mathrm{M} ;[\mathrm{DBU}]=0.8 \mathrm{M} ;[$ HBpin $]=0.88 \mathrm{M} ;[$ DAP-H $]=0.02 \mathrm{M}$ (blue line), $[$ DAP-H $]=0.04 \mathrm{M}$ (orange line), $[\mathbf{D A P}-\mathbf{H}]=0.06 \mathrm{M}$ (grey line), $[\mathbf{D A P}-\mathbf{H}]=0.08 \mathrm{M}$ (blue line). b) Plot of initial rate (mmol/min) versus DAP-H (mmol) with fit to $\mathrm{y}=\mathrm{ax}^{\mathrm{b}}$ where, $\mathrm{y}=\Delta[\mathrm{[} \mathrm{a}] / \Delta[\mathrm{t}, \mathrm{x}=[\mathbf{D A P}-\mathbf{H}], \mathrm{b}=$ reaction order. 
Order dependence in substrate 2a

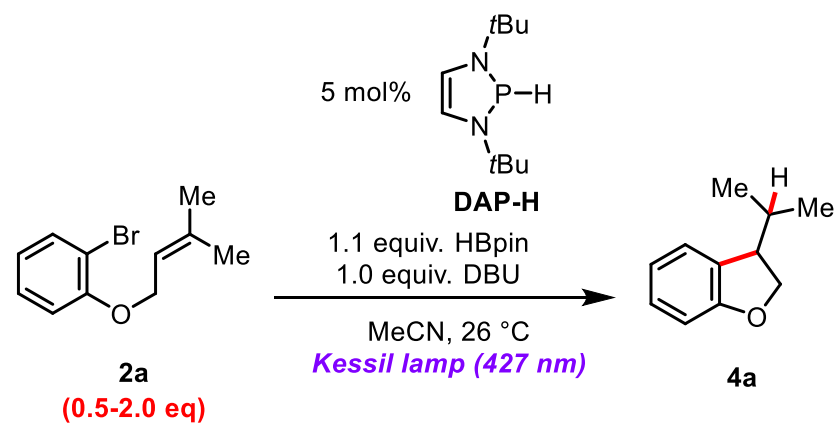

GP B was used with the following modifications: The amount of $\mathbf{2 a}(60.0-242 \mathrm{mg}, 0.25-1.00 \mathrm{mmol}, 0.50-$ 2.00 equiv.) was varied.

a)

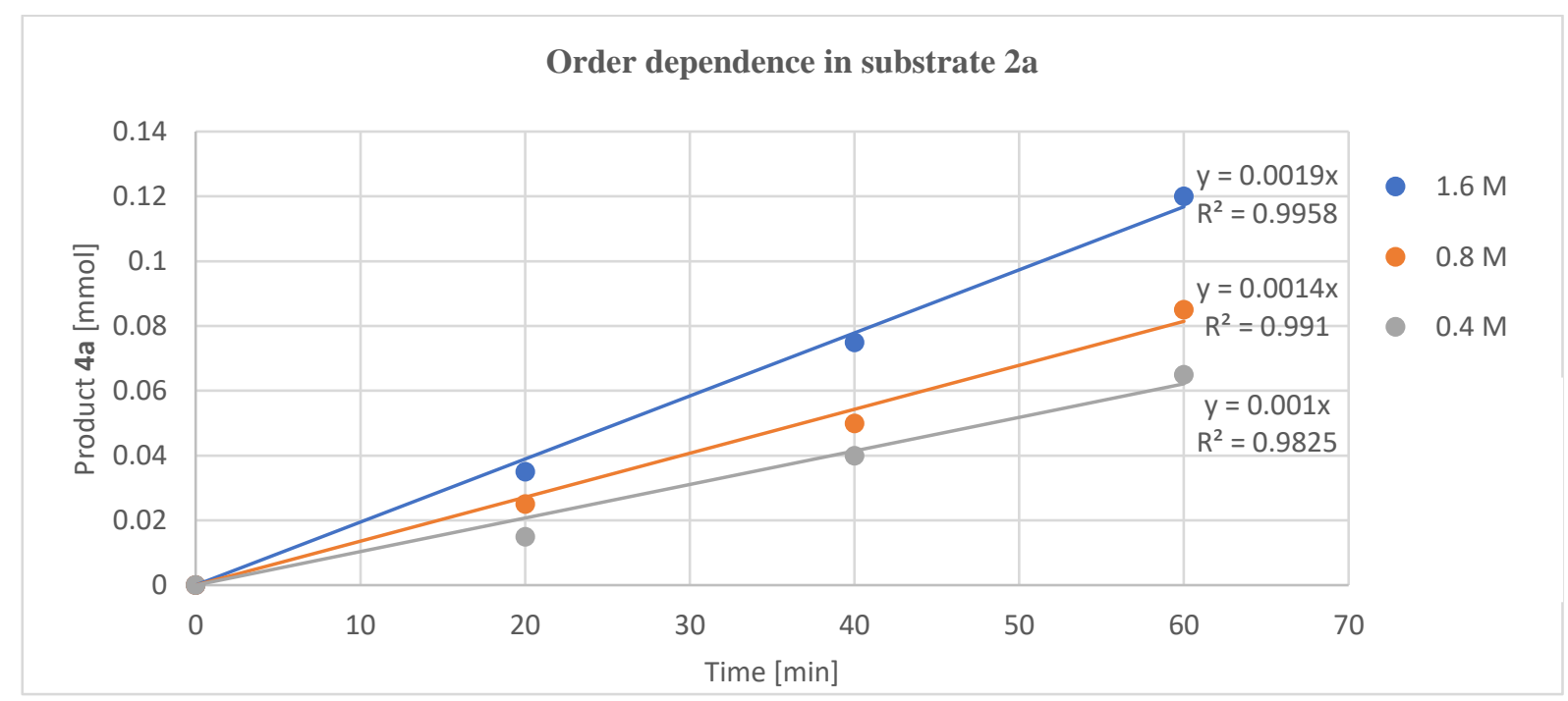

b)

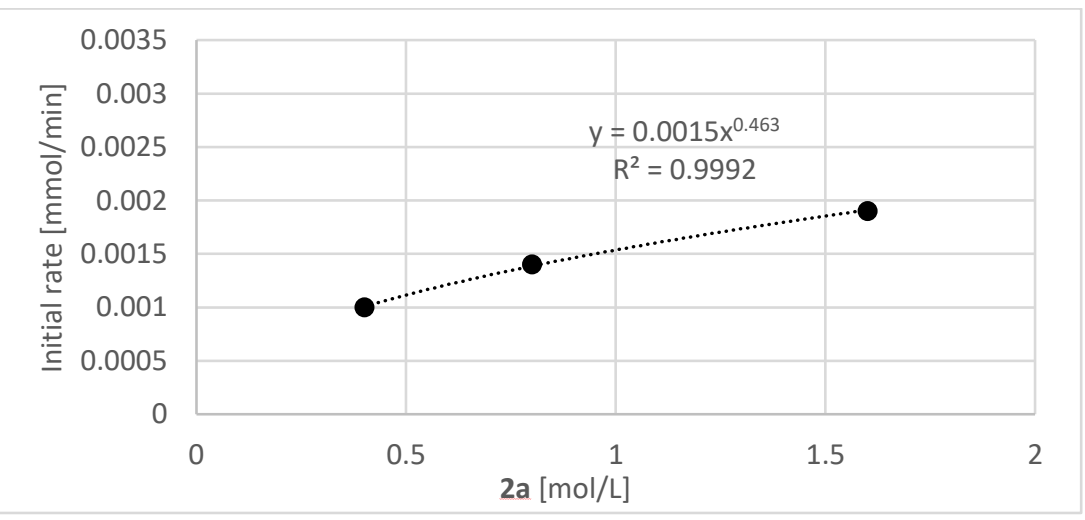

Figure S18: a) Reaction profiles at different initial concentrations of 2a showing a half-order dependence in 2a. Rate constants calculated from the slope of the plots. [DBU] $=0.8 \mathrm{M} ;[\mathrm{HBpin}]=0.88 \mathrm{M} ;[\mathrm{DAP}-\mathrm{H}]=0.04 \mathrm{M} ;[\mathbf{2 a}]=0.40 \mathrm{M}$; (grey line), [2a] $=0.8 \mathrm{M}$; (orange line), [2a]= $1.6 \mathrm{M}$; (blue line). $\mathrm{b}$ ) Plot of initial rate $(\mathrm{mmol} / \mathrm{min})$ versus $1(\mathrm{mmol})$ with fit to $\mathrm{y}=\mathrm{ax}^{\mathrm{b}}$ where, $\mathrm{y}=\Delta[4 \mathrm{a}] / \Delta[\mathrm{t}, \mathrm{x}=[\mathbf{2 a}], \mathrm{b}=\mathrm{reaction}$ order. 


\section{Order dependence in DBU}

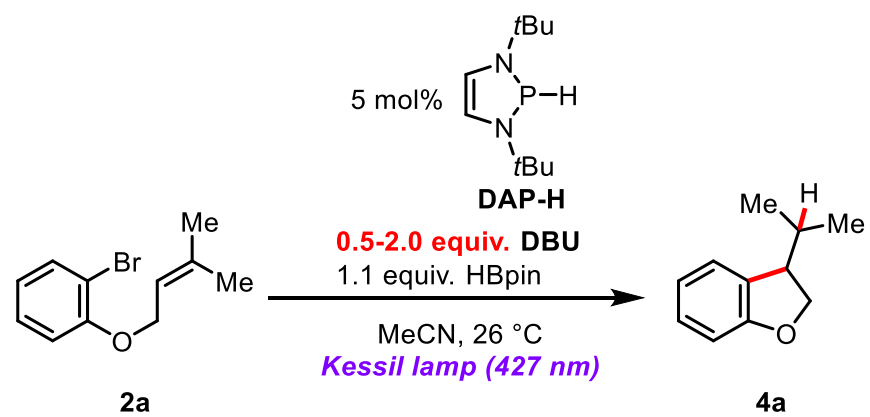

GP B was used with the following modifications: The amount of DBU (38.0 - $152 \mathrm{mg}, 38.0-151 \mu \mathrm{L}, 0.25-1.00$ mmol, $0.5-2.0$ equiv.) was varied.

a)

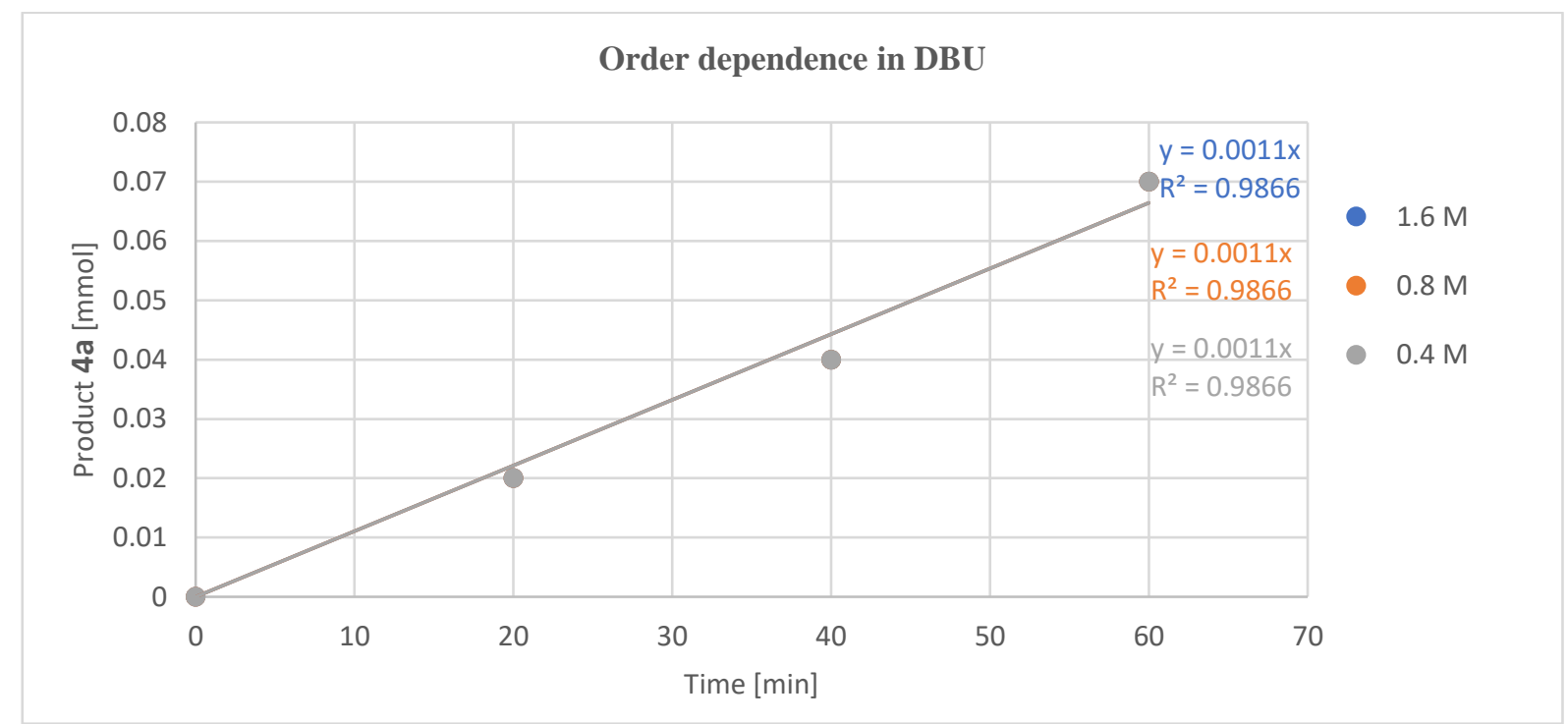

b)

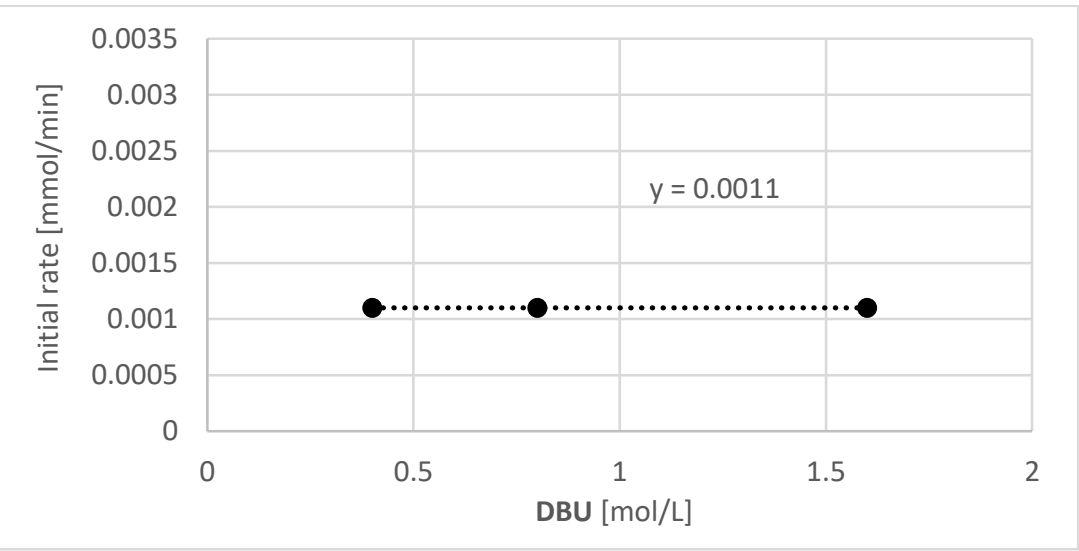

Figure S19: a) Reaction profiles at different initial concentrations of DBU showing a zero-order dependence in DBU. Rate constants calculated from the slope of the plots. $[2 \mathrm{a}]=0.8 \mathrm{M} ;[\mathrm{HBpin}]=0.88 \mathrm{M} ;[\mathrm{DAP}-\mathrm{H}]=0.04 \mathrm{M}$; $[\mathbf{D B U}]=0.40 \mathrm{M}$ (grey line), [DBU]=0.8 M; (orange line), $[\mathbf{D B U}]=1.6 \mathrm{M}$; (blue line). b) Plot of initial rate $(\mathrm{mmol} / \mathrm{min})$ versus DBU $(\mathrm{mmol})$ with fit to $\mathrm{y}=\mathrm{ax}{ }^{\mathrm{b}}$ where, $\mathrm{y}=\Delta[4 \mathrm{a}] / \Delta \mathrm{t}, \mathrm{x}=$ [DBU], b = reaction order. 


\section{Order dependence in HBpin}

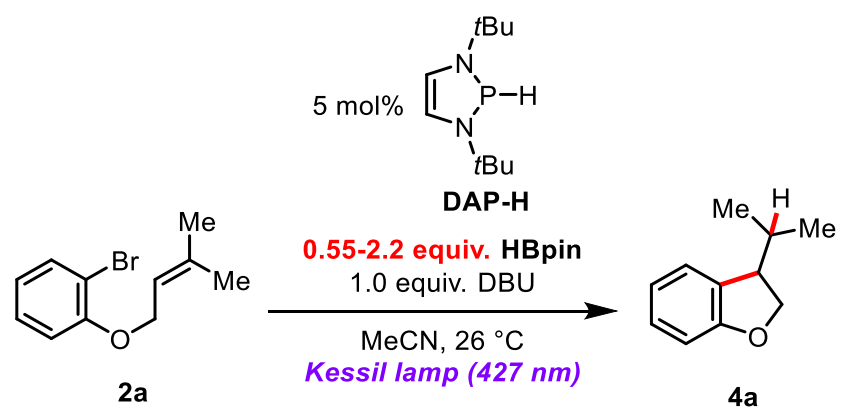

GP B was used with the following modifications: The amount of HBpin $(35.0-141 \mathrm{mg}, 40.0-160 \mu \mathrm{L}, 0.275-$ $1.10 \mathrm{mmol}, 0.55-2.20$ equiv.) was varied.

a)

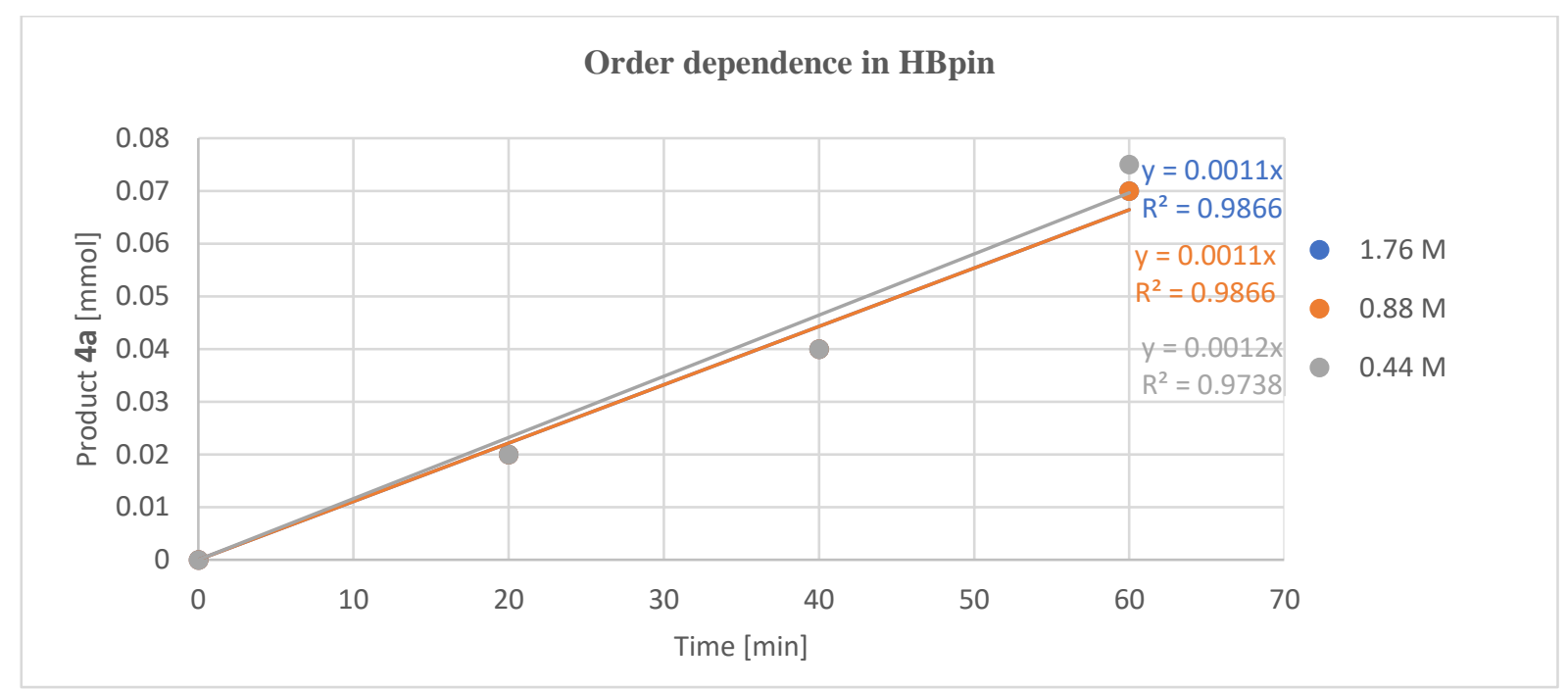

b)

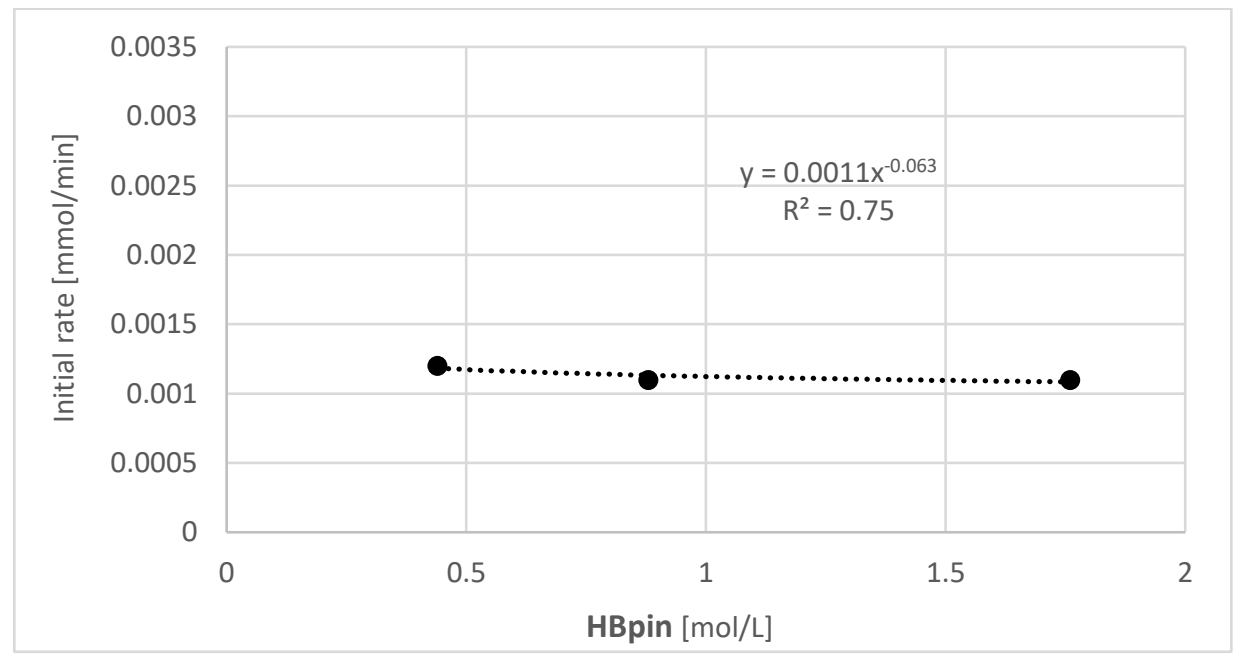

Figure S20: a) Reaction profiles at different initial concentrations of HBpin showing a zero-order dependence in HBpin. Rate constants calculated from the slope of the plots. $[2 \mathrm{a}]=0.80 \mathrm{M} ;[\mathrm{DBU}]=0.8 \mathrm{M} ;[\mathrm{DAP}-\mathrm{H}]=0.04 \mathrm{M} ;[$ HBpin $]=0.44 \mathrm{M}$ (grey line), $[$ HBpin $]=0.88$ $\mathrm{M}$; (orange line), [HBpin] = $1.76 \mathrm{M}$; (blue line). b) Plot of initial rate $\left(\mathrm{mmol} / \mathrm{min}\right.$ ) versus HBpin (mmol) with fit to $\mathrm{y}=\mathrm{ax}^{\mathrm{b}}$ where, $\mathrm{y}=$ $\Delta[4 \mathrm{a}] / \Delta \mathrm{t}, \mathrm{x}=[$ HBpin $], \mathrm{b}=$ reaction order. 


\section{Effect of the $(\mathbf{D A P})_{2}$ additive on the stoichiometric reaction}

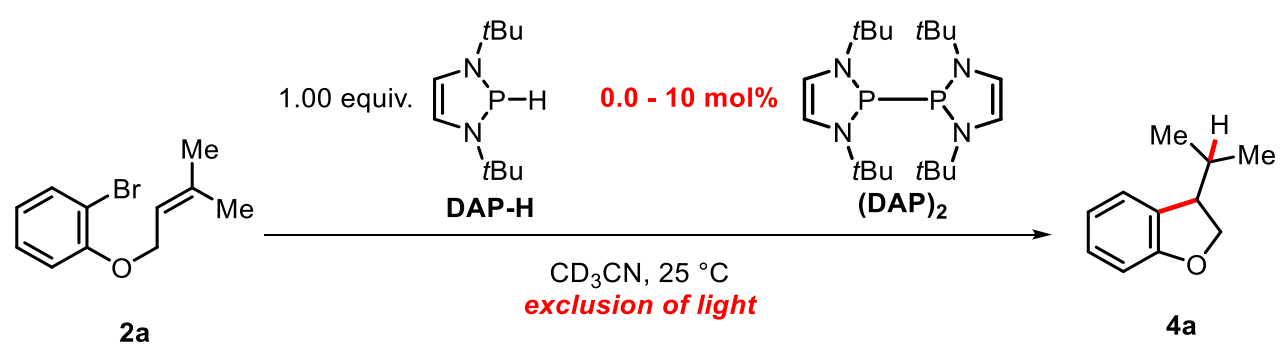

A dried J-Young NMR tube was charged with substrate 2a (120.6 mg, $0.50 \mathrm{mmol}, 1.00$ equiv.) and 1,3,5trimethoxybenzene $(28.0 \mathrm{mg}, 0.17 \mathrm{mmol}, 0.33$ equiv.) under air and the tube was introduced inside the glovebox. Next, dry and degassed $\mathrm{CD}_{3} \mathrm{CN}(0.625 \mathrm{ml})$ was added followed by addition of DAP-H $(100 \mathrm{mg}, 0.50 \mathrm{mmol}$, 1.00 equiv.) and (DAP) $)_{2}(0.0-10 \mathrm{~mol} \%)$ under exclusion of light. The tube sealed and the reaction progress was monitored by ${ }^{1} \mathrm{H}$ NMR.

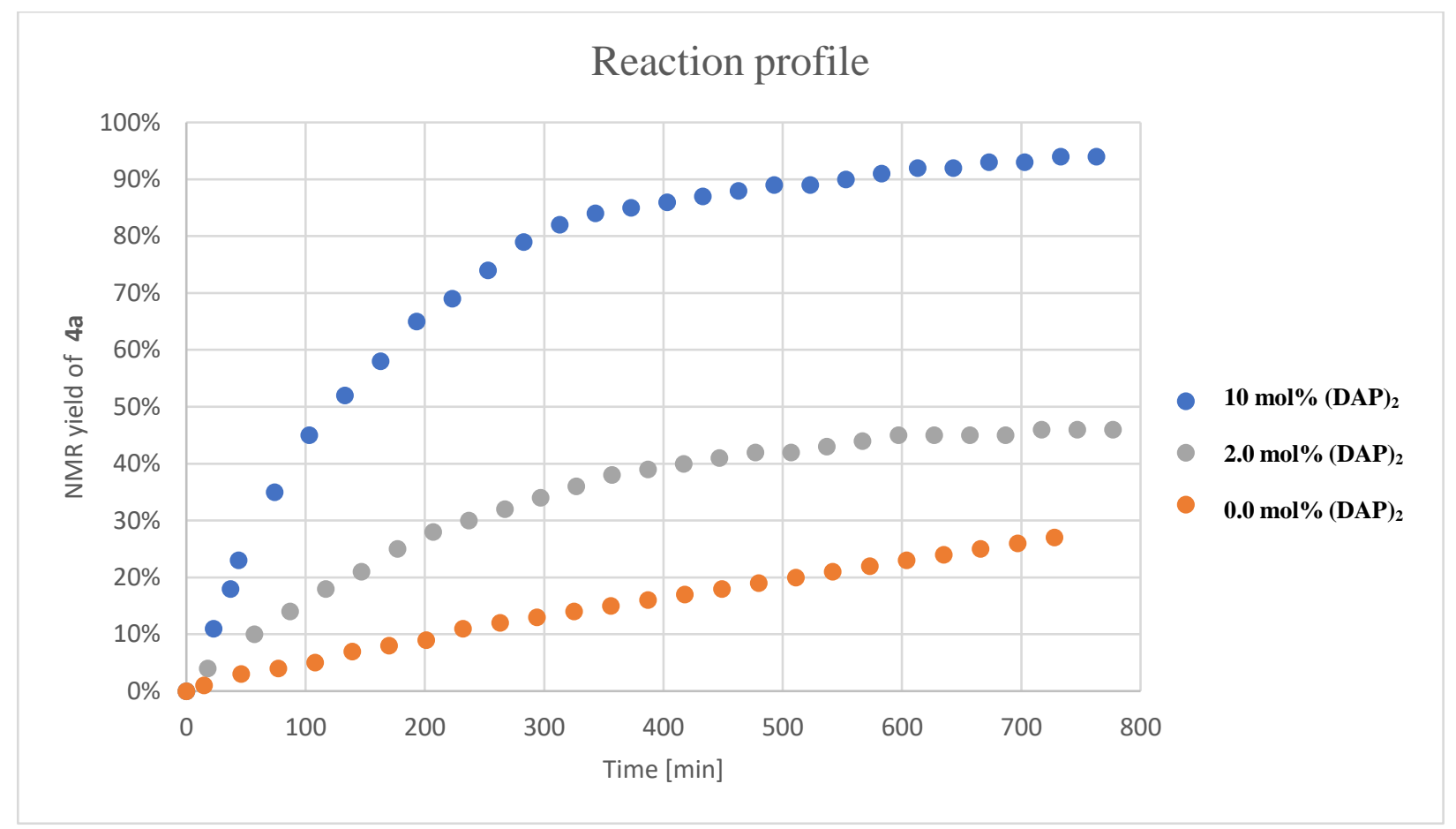

Figure S21: Reaction profiles with different amounts of $(\mathbf{D A P})_{2}$. [2a $]=0.8 \mathrm{M} ;[\mathbf{D A P}-\mathbf{H}]=0.8 \mathrm{M} ;(\mathbf{D A P})_{2}=0.0$ mol\%; (orange line), $(\mathbf{D A P})_{2}$ $=2.0 \mathrm{~mol} \%$; (grey line), $(\mathbf{D A P})_{2}=10 \mathrm{~mol} \%$; (blue line) . 
Dependance in light.

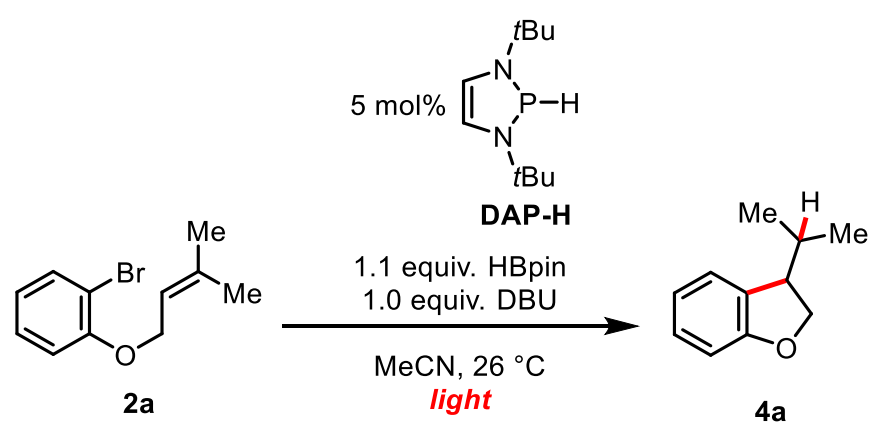

GP B was used with the following modifications: The power of the light source was varied.

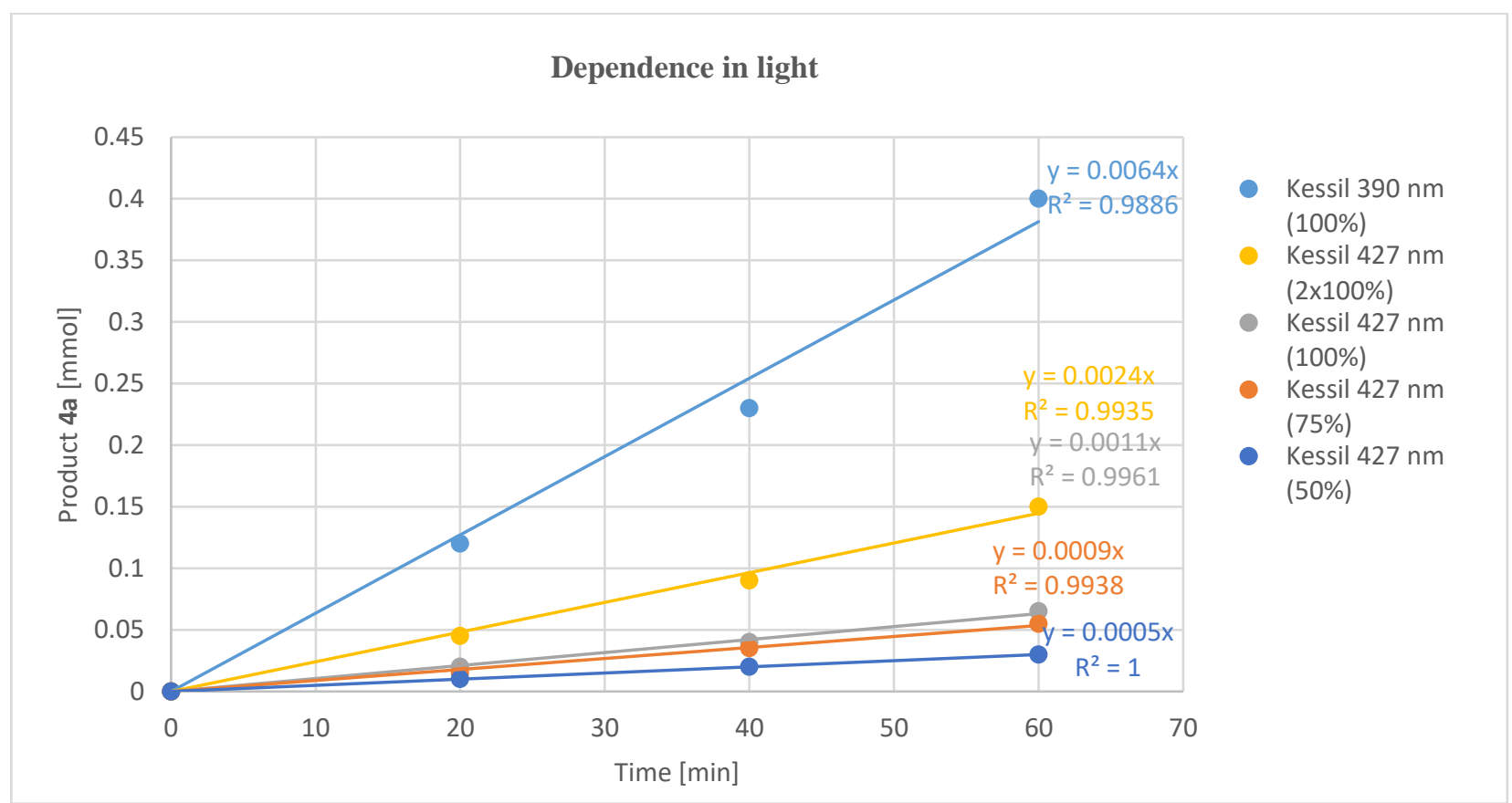

Figure S22: Reaction profiles at different irradiance. [2a] = $0.8 \mathrm{M}$; [DBU] = $0.8 \mathrm{M} ;[$ DAP-H $]=0.04$; [HBpin] = $0.88 \mathrm{M}$. Kessil lamp (427 $\mathrm{nm}$ ) $50 \%$ power (dark blue line), Kessil lamp (427 nm) 75\% power (orange line), Kessil lamp (427 nm) $100 \%$ power (grey line), $2 \times$ Kessil lamp (427 nm) 100\% power (yellow line), Kessil lamp (390 nm) 100\% power (light blue line). 


\subsubsection{Deuterated experiments}

\section{Exclusion of HAT from the Solvent}

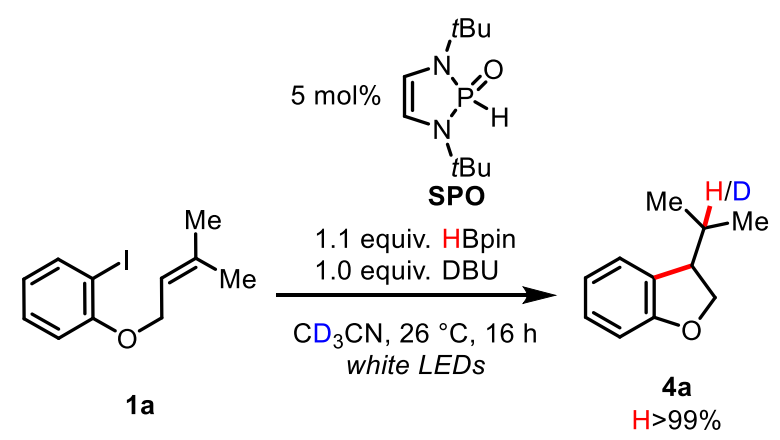

A stirred mixture of $\mathbf{1 a}(28.8 \mathrm{mg}, 100 \mu \mathrm{mol}, 1.00$ equiv.), SPO (1.08 mg, $5.00 \mu \mathrm{mol}, 0.05$ equiv.), DBU (15.2 mg, $100 \mu \mathrm{mol}, 1.00$ equiv.) and $\mathrm{HBpin}\left(14.1 \mathrm{mg}, 110 \mu \mathrm{mol}, 1.10\right.$ equiv.) in $\mathrm{CD}_{3} \mathrm{CN}(1 \mathrm{~mL})$ was irradiated with white LEDs for $16 \mathrm{~h}$. Volatiles were removed under reduced pressure and the residue was purified by flash chromatography ( $n$-pentane:EA=40:1) to afford 4a as a colorless oil. A $\mathrm{H}$ incorporation greater than $99 \%$ was determined by ${ }^{1} \mathrm{H}$ NMR.

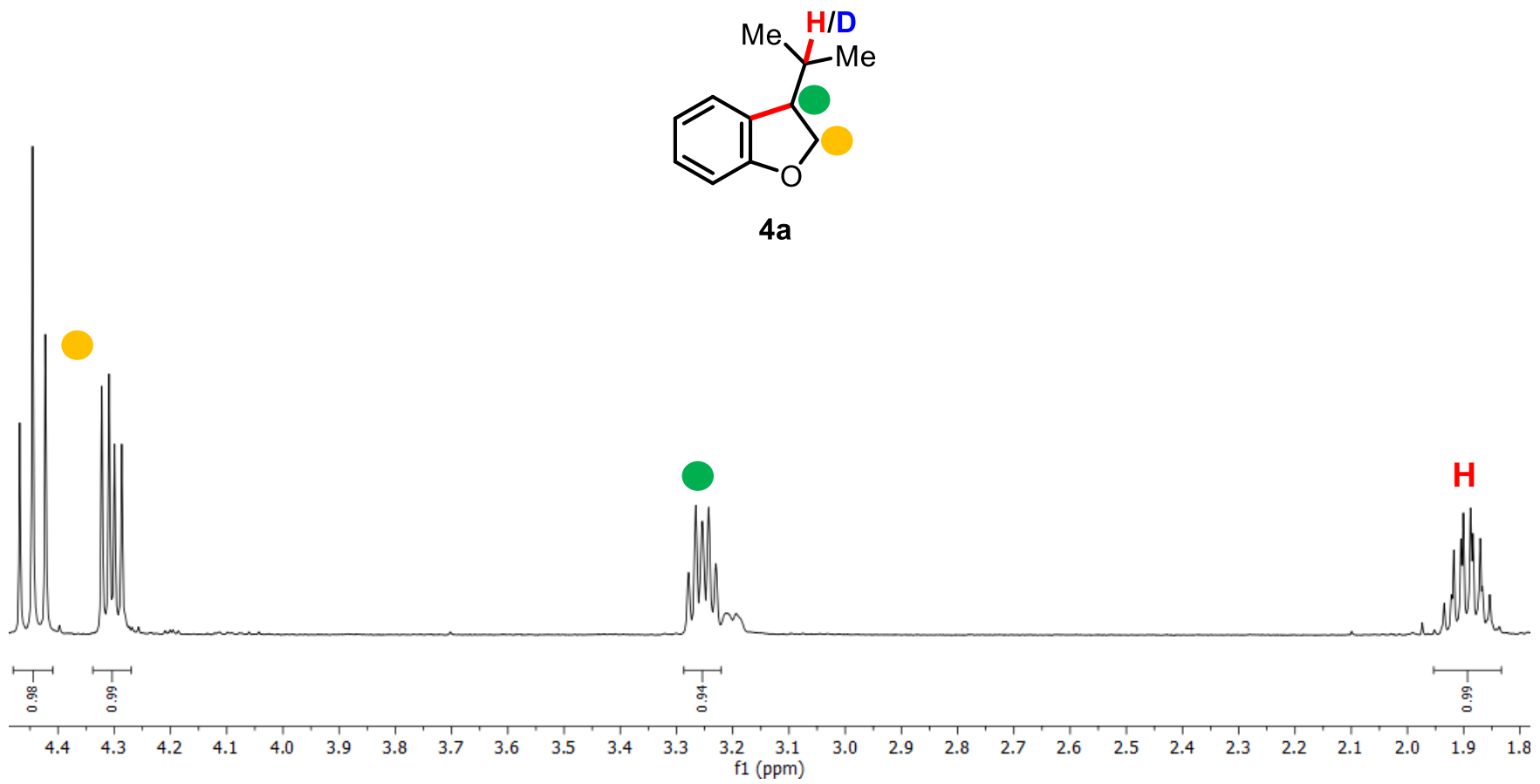

Figure S23: Zoom of the ${ }^{1} \mathrm{H}$ NMR spectra of isolated 4a. 


\section{Proof of possible HAT from a DAP species}

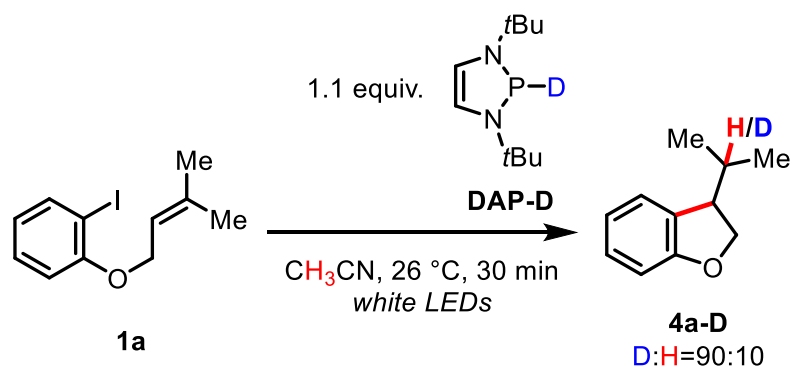

A stirred mixture of 1a (57.6 mg, $0.20 \mathrm{mmol}, 1.00$ equiv.) and DAP-D (44.3 mg, $0.22 \mathrm{mmol}, 1.10$ equiv.) in MeCN $(2 \mathrm{~mL})$ was irradiated with white LEDs for $30 \mathrm{~min}$. Volatiles were removed under reduced pressure and the residue was purified by flash chromatography ( $n$-pentane:EA=40:1) to afford 4a-D as a colorless oil. An incorporation of $\mathrm{D}: \mathrm{H}=90: 10$ was determined by ${ }^{1} \mathrm{H}$ NMR.

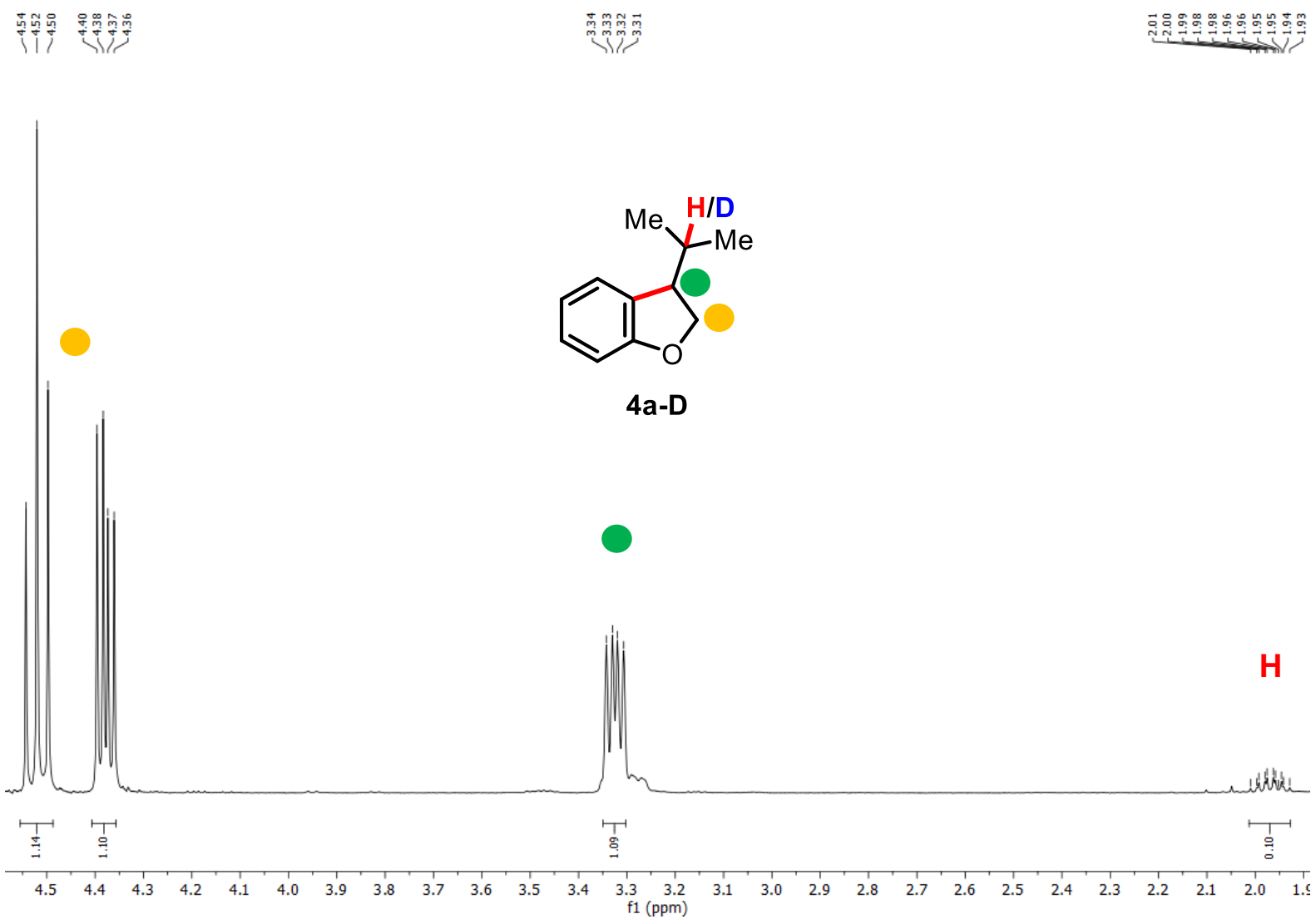

Figure S24: Zoom of the ${ }^{1} \mathrm{H}$ NMR spectra of isolated 4a-D 


\subsubsection{Formation of $(\mathrm{DAP})_{2}$ from DAP-H under Irradiation}<smiles></smiles>

DAP-H

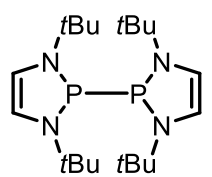

$(\mathrm{DAP})_{2}$

A dried J-Young NMR tube was charged with a solution of DAP-H $(10 \mathrm{mg}, 50 \mu \mathrm{mol})$ in $\mathrm{CD}_{3} \mathrm{CN}(0.5 \mathrm{~mL})$ inside the glovebox. The tube was sealed and submitted to NMR analysis. In ${ }^{31}$ P NMR, only DAP-H was detected at $\delta=56.86 \mathrm{ppm}$ (Figure S25a). The tube was irradiated with a Kessil lamp (427 nm) for $5 \mathrm{~h}$. After irradiation, a solid precipitate was observed, presumably $(\mathbf{D A P})_{2}$, which was found to be poorly soluble in $\mathrm{MeCN}$ and $\mathrm{CD}_{3} \mathrm{CN}$. ${ }^{31} \mathrm{P}$ NMR analysis showed complete conversion of DAP-H and the new signal at $\delta=78.20 \mathrm{ppm}$ corresponded to $($ DAP) 2 (Figure S25Figure S25 b).

\section{a)}
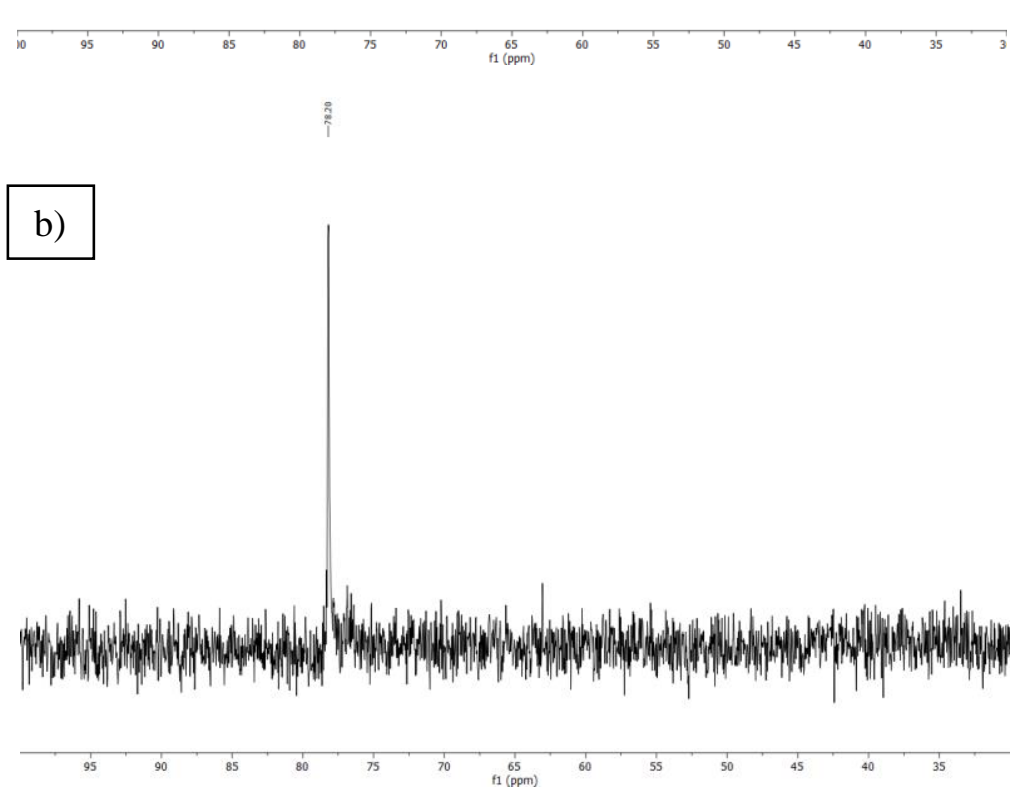

Figure S25: ${ }^{31} \mathrm{P}$ NMR spectra of a solution of DAP-H in $\mathrm{CD}_{3} \mathrm{CN}$ a) before and b) after irradiation with Kessil lamp (427 nm) to access $(\mathbf{D A P})_{2}$. 


\subsubsection{Attempts to inhibit the reductive cyclization by TEMPO}

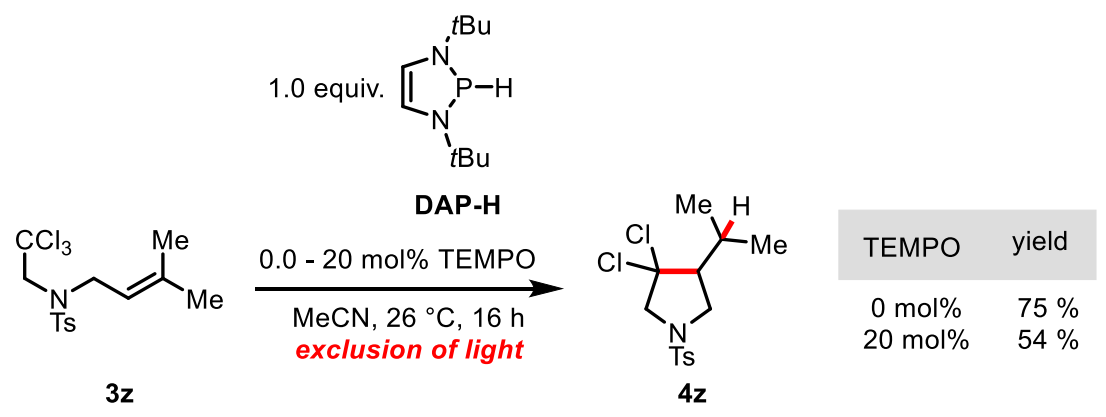

Two separate $1 \mathrm{~mL}$ microwave vial, equipped with a stir bar were charged with $\mathbf{3 z}$ (37.1 mg, $100 \mu \mathrm{mol}$, 1.00 equiv.), to one vial was added (2,2,6,6-tetramethylpiperidin-1-yl)oxyl (TEMPO) (3.13 mg, $20 \mu \mathrm{mol}, 0.20$ equiv.) and both vials were transferred into the glovebox. MeCN $(125 \mu \mathrm{L})$ was added, followed by DAP-H $(20.0 \mathrm{Mg}, 100 \mu \mathrm{mol}$, 1.00 equiv.) under strict exclusion of light. The vials were capped and the reaction mixtures were stirred for $16 \mathrm{~h}$ at $26{ }^{\circ} \mathrm{C}$. Volatiles were removed under reduced pressure and the residue was directly subjected to ${ }^{1} \mathrm{H}$ NMR analysis with 1,3,5-trimethoxybenzene as an internal standard.

Note: DAP-H reacts rapidly with TEMPO, thus inhibition of hypothesized radical intermediates could not be observed. 


\subsection{DAP Synthesis}

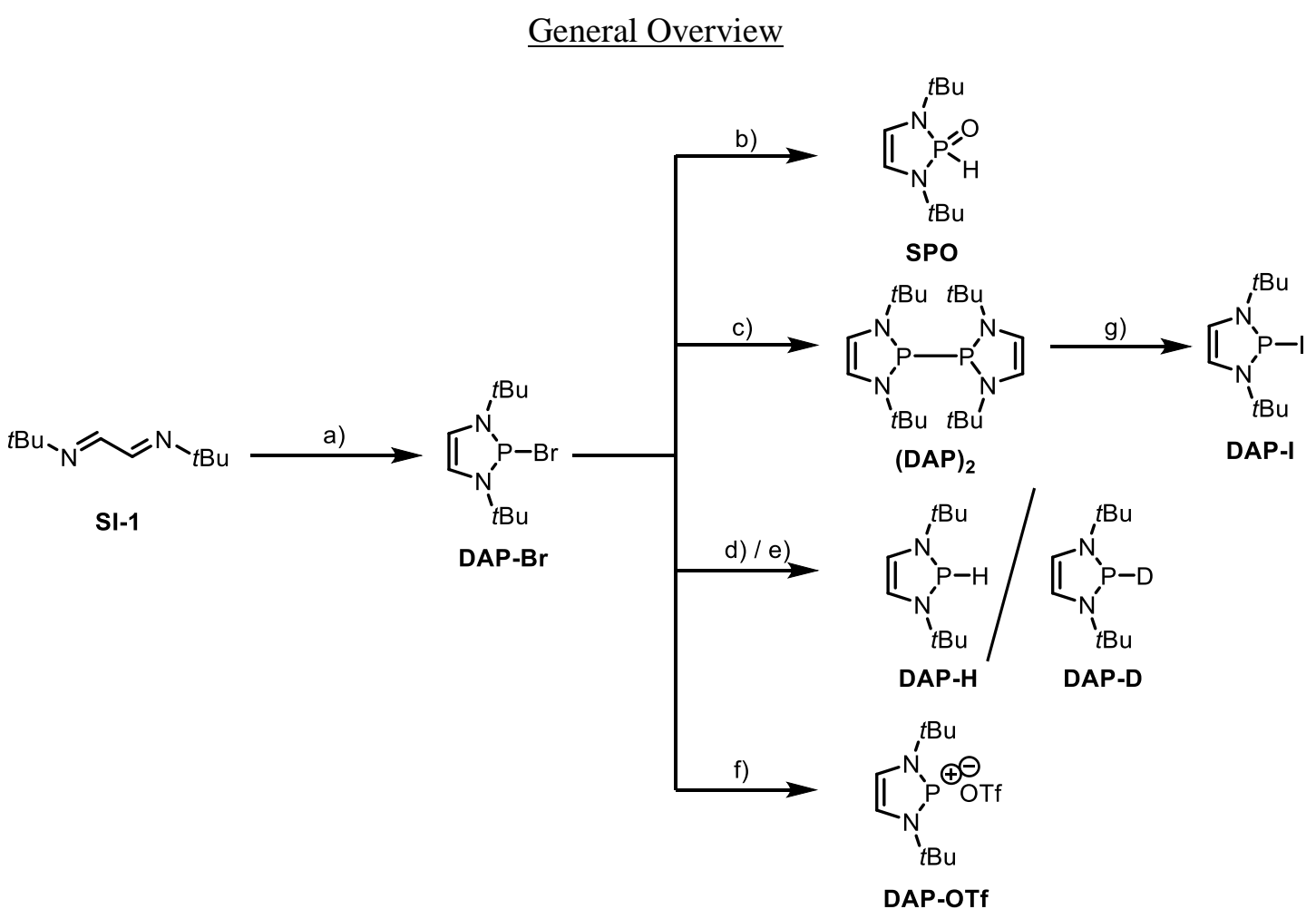

$N^{1}, N^{2}$-Di-tert-butylethane-1,2-diimine (SI-1)

$$
{ }_{\mathrm{SI}-1}^{t \mathrm{Bu}} \mathrm{N}_{\mathrm{N}}=\mathrm{N}_{t \mathrm{Bu}}
$$

To a stirred solution of oxaldehyde ( $2.90 \mathrm{~g}, 20.0 \mathrm{mmol}, 1.00$ equiv.) in $\mathrm{H}_{2} \mathrm{O}$ (20 w-\%) was added tert-butylamine $\left(2.09 \mathrm{~mL}, 1.46 \mathrm{~g}, 20.0 \mathrm{mmol}, 2.00\right.$ equiv.) at $0^{\circ} \mathrm{C}$ under air. Instantly a white precipitate formed and the reaction mixture solidified. The mixture was sonicated for 15 min. The solid was filtered, washed several times with $\mathrm{H}_{2} \mathrm{O}$, dissolved in $\mathrm{CH}_{2} \mathrm{Cl}_{2}(20 \mathrm{~mL})$ and dried over $\mathrm{Na}_{2} \mathrm{SO}_{4}$. All volatiles were removed under reduced pressure to afford SI-1 (1.19 g, $7.04 \mathrm{mmol}, 70 \%)$ as a white solid with a wet appearance, which was used in the next step without further purification.

Analytical data of SI-1:

${ }^{1}$ H NMR (400 MHz, $\mathrm{CDCl}_{3}$ ): $\delta=7.93$ (s, 2H), 1.26 (s, 18H) ppm.

${ }^{13} \mathrm{C}$ NMR (100 MHz, $\left.\mathrm{CDCl}_{3}\right): \delta=158.1,58.4,29.5 \mathrm{ppm}$.

All spectroscopic data corresponded to the reported literature values. ${ }^{[3]}$ 


\section{2-Bromo-1,3-di-tert-butyl-2,3-dihydro-1H-1,3,2-diazaphosphole (DAP-Br)}

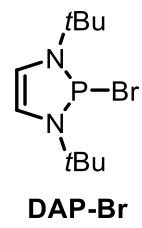

a) To a stirred solution of diimine SI-1 (505 mg, $3.00 \mathrm{mmol}, 1.00$ equiv.) in dry $\mathrm{Et}_{2} \mathrm{O}(5 \mathrm{~mL})$ was added cyclohexene $\left(912 \mu \mathrm{L}, 739 \mathrm{mg}, 9.00 \mathrm{mmol}, 3.00\right.$ equiv.), followed by $\mathrm{PBr}_{3}(285 \mu \mathrm{L}, 812 \mathrm{mg}, 3.00 \mathrm{mmol}$, 1.00 equiv.) at rt. The mixture was stirred for $20 \mathrm{~h}$ at $\mathrm{rt}$ and all volatiles were removed in vacuo. The solid residue was transferred into the glovebox, filtered, and washed with $\mathrm{Et}_{2} \mathrm{O}$ to afford the title compound $(623 \mathrm{mg}, 2.23 \mathrm{mmol}$, $74 \%)$ as a faint yellow powder.

Analytical data of $\mathbf{D A P}-\mathbf{B r}$ :

${ }^{1} \mathbf{H}$ NMR $\left(400 \mathrm{MHz}, \mathrm{CDCl}_{3}\right): \delta=7.21(\mathrm{~s}, 2 \mathrm{H}), 1.73(\mathrm{~s}, 19 \mathrm{H}) \mathrm{ppm}$.

${ }^{13}$ C NMR (100 MHz, $\left.\mathrm{CDCl}_{3}\right): \delta=124.3(\mathrm{~d}, J=7.2 \mathrm{~Hz}), 59.3(\mathrm{~d}, J=7.4 \mathrm{~Hz}), 30.3(\mathrm{~d}, J=10.0 \mathrm{~Hz}) \mathrm{ppm}$.

${ }^{31} \mathbf{P}$ NMR $\left(162 \mathrm{MHz}, \mathrm{CDCl}_{3}\right): \delta=185.89 \mathrm{ppm}$.

All spectroscopic data corresponded to the reported literature values. ${ }^{[4]}$

\section{1,3-Di-tert-butyl-1,3-dihydro-1,3,2-diazaphosphole 2-oxide (SPO)}

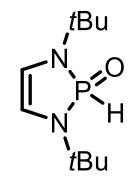

SPO

b) SPO was synthesized according to a known procedure. ${ }^{[5]}$ To a stirred solution of DAP-Br (500 mg, 1.79 mmol, 1.00 equiv. $)$ in $\mathrm{CH}_{2} \mathrm{Cl}_{2}(5 \mathrm{~mL})$ was added $\mathrm{NEt}_{3}(250 \mu \mathrm{L}, 181 \mathrm{mg}, 1.79 \mathrm{mmol}, 1.00$ equiv.) at rt. The resulting mixture was stirred for $5 \mathrm{~min}$ and quenched by the addition of $\mathrm{H}_{2} \mathrm{O}(10 \mathrm{~mL})$. After stirring for $1 \mathrm{~h}$, the reaction mixture was extracted with $\mathrm{CH}_{2} \mathrm{Cl}_{2}(2 \times 15 \mathrm{~mL})$. Organic layers were dried over $\mathrm{Na}_{2} \mathrm{SO}_{4}$ and all volatiles were removed under reduced pressure to afford the title compound (349 $\mathrm{mg}, 1.61 \mathrm{mmol}, 90 \%)$ as an ivory solid.

Analytical data of SPO:

${ }^{1} \mathrm{H}$ NMR $\left(400 \mathrm{MHz}, \mathrm{CDCl}_{3}\right): \delta=8.60(\mathrm{dt}, J=646.9,1.8 \mathrm{~Hz}, 1 \mathrm{H}), 5.95(\mathrm{dd}, J=16.0,1.7 \mathrm{~Hz}, 2 \mathrm{H}), 1.43(\mathrm{~s}, 18 \mathrm{H})$ ppm.

${ }^{13}$ C NMR $\left(100 \mathrm{MHz}, \mathrm{CDCl}_{3}\right): \delta=110.6(\mathrm{~d}, J=10.2 \mathrm{~Hz}), 53.6(\mathrm{~d}, J=4.3 \mathrm{~Hz}), 30.2(\mathrm{~d}, J=4.0 \mathrm{~Hz})$

${ }^{31} \mathbf{P}$ NMR $\left(162 \mathrm{MHz}, \mathrm{CDCl}_{3}\right): \delta=3.36 \mathrm{ppm}$.

All spectroscopic data corresponded to the reported literature values. ${ }^{[5]}$ 


\section{1,1',3,3'-Tetra-tert-butyl-1,1',3,3'-tetrahydro-2,2'-bi(1,3,2-diazaphosphole) $(\mathrm{DAP})_{2}$}

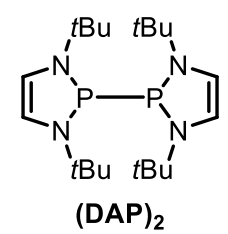

c) $(\mathbf{D A P})_{2}$ was synthesized according to a known procedure. ${ }^{[6]}$ To a stirred suspension of DAP-Br $(4.02 \mathrm{~g}$, $14.4 \mathrm{mmol}, 1.00$ equiv.) and $\mathrm{Mg}$ (turnings, $1.05 \mathrm{~g}, 43.2 \mathrm{mmol}, 3.00$ equiv.) in dry THF (12 mL) inside a Schlenk flask, was added iodine ( $366 \mathrm{mg}, 1.44 \mathrm{mmol}, 0.10$ equiv.) at rt. The mixture was stirred for $4 \mathrm{~h}$ and all volatiles were removed in vacuo. The flask was transferred into the glovebox and the residue was suspended in $n$-pentane $(20 \mathrm{~mL})$. The resulting mixture was filtered over celite and the remaining solid was washed with $n$-pentane $(2 \times 20$ $\mathrm{mL})$. All volatiles were removed in vacuo to afford the title compound $(2.55 \mathrm{~g}, 6.41 \mathrm{mmol}, 44 \%)$ as an off-white solid.

Analytical data of $(\boldsymbol{D A P})_{2}$ :

${ }^{1} \mathbf{H}$ NMR $\left(400 \mathrm{MHz}, \mathrm{C}_{6} \mathrm{D}_{6}\right): \delta=5.94(\mathrm{t}, J=1.9 \mathrm{~Hz}, 4 \mathrm{H}), 1.26(\mathrm{~s}, 36 \mathrm{H}) \mathrm{ppm}$.

${ }^{13}$ C NMR (100 MHz, $\left.\mathrm{C}_{6} \mathrm{D}_{6}\right): \delta=121.3,54.2(\mathrm{t}, J=7.7 \mathrm{~Hz}), 30.3(\mathrm{t}, J=4.0 \mathrm{~Hz}) \mathrm{ppm}$.

${ }^{31} \mathbf{P}$ NMR $\left(162 \mathrm{MHz}, \mathrm{C}_{6} \mathrm{D}_{6}\right): \delta=79.86 \mathrm{ppm}$.

${ }^{1} \mathbf{H}$ NMR (400 MHz, $\left.\mathrm{CD}_{3} \mathrm{CN}\right): \delta=5.95(\mathrm{t}, J=2.0 \mathrm{~Hz}, 4 \mathrm{H}), 1.18(\mathrm{~s}, 36 \mathrm{H}) \mathrm{ppm}$.

${ }^{31} \mathbf{P}$ NMR $\left(162 \mathrm{MHz}, \mathrm{CD}_{3} \mathrm{CN}\right): \delta=78.20 \mathrm{ppm}$.

All spectroscopic data (acquired in $\mathrm{C}_{6} \mathrm{D}_{6}$ ) corresponded to the reported literature values. ${ }^{[6]}$

1,3-Di-tert-butyl-2,3-dihydro-1H-1,3,2-diazaphosphole (DAP-H)<smiles>[132In]n1ccnc1</smiles>

DAP-H

d) To a stirred suspension of DAP-Br ( $2.70 \mathrm{~g}, 10.0 \mathrm{mmol}, 1.00$ equiv. $)$ in dry and degassed THF (70 mL) inside a Schlenk flask was added $\mathrm{LiAlH}_{4}(1.25 \mathrm{~mL}, 2.4 \mathrm{M}$ in THF, $114 \mathrm{mg}, 3.00 \mathrm{mmol}, 0.30$ equiv. $)$ at $-78{ }^{\circ} \mathrm{C}$. The mixture was stirred for $30 \mathrm{~min}$ at $-78^{\circ} \mathrm{C}$. The cooling bath was removed and the mixture was stirred for further $2 \mathrm{~h}$. All volatiles were removed in vacuo and the flask was transferred into the glovebox. The residue was suspended in $n$-hexane $(15 \mathrm{~mL})$ and filtered over celite. The remaining residue inside the flask was washed with additional $n$-hexane $(2 \times 20 \mathrm{~mL})$. Volatiles were removed in vacuo to afford a yellow oil. Distillation $\left(1 \mathrm{mbar}, 73^{\circ} \mathrm{C}\right)$ afforded the title compound (1.19 g, $5.94 \mathrm{mmol}, 59 \%)$ as a faint yellow liquid.

Analytical data of DAP-H:

${ }^{1}$ H NMR $\left(400 \mathrm{MHz}, \mathrm{C}_{6} \mathrm{D}_{6}\right): \delta=6.16(\mathrm{~d}, J=184.2 \mathrm{~Hz}, 1 \mathrm{H}), 6.00(\mathrm{~d}, J=3.9 \mathrm{~Hz}, 2 \mathrm{H}), 1.16(\mathrm{~s}, 18 \mathrm{H}) \mathrm{ppm}$.

${ }^{31} \mathbf{P}$ NMR $\left(162 \mathrm{MHz}, \mathrm{C}_{6} \mathrm{D}_{6}\right): \delta=57.49 \mathrm{ppm}$.

${ }^{1}$ H NMR $\left(400 \mathrm{MHz}, \mathrm{CD}_{3} \mathrm{CN}\right): \delta=6.08(\mathrm{~d}, J=4.0 \mathrm{~Hz}, 2 \mathrm{H}), 1.21(\mathrm{~d}, J=1.0 \mathrm{~Hz}, 18 \mathrm{H}) \mathrm{ppm}$.

${ }^{31} \mathbf{P}$ NMR $\left(162 \mathrm{MHz}, \mathrm{CD}_{3} \mathrm{CN}\right): \delta=56.81 \mathrm{ppm}$.

All spectroscopic data in $\mathrm{C}_{6} \mathrm{D}_{6}{ }^{[7]}$ and $\mathrm{CD}_{3} \mathrm{CN}^{[8]}$ corresponded to the reported literature values. 


\section{1,3-Di-tert-butyl-2,3-dihydro-1H-1,3,2-diazaphosphole-2-d (DAP-D)}

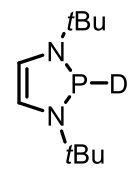

DAP-D

e) A solution of $\mathrm{LiAlD}_{4}$ was prepared by a modified literature procedure ${ }^{[9]}$ :

Inside the glovebox, $\mathrm{LiD}$ (186 mg, $20.6 \mathrm{mmol}, 2.06$ equiv.) and $\mathrm{LiAlD}_{4}$ (23.6 mg, $0.56 \mathrm{mmol}, 0.06$ equiv.) were thoroughly ground separately and suspended in $\mathrm{Et}_{2} \mathrm{O}(2 \mathrm{~mL})$. Under stirring, a solution of $\mathrm{AlCl}_{3}(500 \mathrm{mg}$, $3.75 \mathrm{mmol}, 0.38$ equiv.) in $\mathrm{Et}_{2} \mathrm{O}(5 \mathrm{~mL})$ was added dropwise. The reaction was stirred for further $30 \mathrm{~min}$ and filtered over a short plug of celite. This solution of $\mathrm{LiAlD}_{4}$ was used without further analysis or purification in the next step.

Outside the glovebox, the prior obtained solution of $\mathrm{LiAlD}_{4}$ in $\mathrm{Et}_{2} \mathrm{O}$ was added to a stirred solution of DAP-Br $(2.79 \mathrm{~g}, 10.0 \mathrm{mmol}, 1.00$ equiv. $)$ in THF $(70 \mathrm{~mL})$ inside a Schlenk tube at $-78^{\circ} \mathrm{C}$. The mixture was stirred for further $30 \mathrm{~min}$ at this temperature and was allowed to reach $\mathrm{rt}$, at which the stirring was continued for additional $2 \mathrm{~h}$. All volatiles were removed in vacuo and the Schlenk tube was transferred into the glovebox. The yellow residue was suspended in $n$-hexane $(15 \mathrm{~mL})$, filtered over celite and washed with $n$-hexane $(2 \times 15 \mathrm{~mL})$. The volatiles were removed in vacuo and the remaining yellow liquid residue was distilled under reduced pressure $\left(0.08 \mathrm{mbar}, 70^{\circ} \mathrm{C}\right)$ to afford the title compound as a highly air and moisture sensitive, faint yellow liquid (657 mg, $3.28 \mathrm{mmol}$, 33\%, D incorporation $>95 \%$ )

Analytical data of DAP-D:

${ }^{1} \mathbf{H}$ NMR $\left(400 \mathrm{MHz}, \mathrm{C}_{6} \mathrm{D}_{6}\right): \delta=6.00(\mathrm{~d}, J=4.0 \mathrm{~Hz}, 2 \mathrm{H}), 1.16(\mathrm{~d}, J=1.0 \mathrm{~Hz}, 18 \mathrm{H}) \mathrm{ppm}$.

${ }^{13}$ C NMR (101 MHz, $\left.\mathrm{C}_{6} \mathrm{D}_{6}\right): \delta=121.1(\mathrm{~d}, J=6.8 \mathrm{~Hz}), 53.7(\mathrm{~d}, J=13.5 \mathrm{~Hz}), 29.7(\mathrm{~d}, J=8.8 \mathrm{~Hz}) \mathrm{ppm}$.

${ }^{31} \mathbf{P}$ NMR (162 MHz, $\left.\mathrm{C}_{6} \mathrm{D}_{6}\right): \delta=55.84-55.47(\mathrm{~m}) \mathrm{ppm}$.

${ }^{1} \mathbf{H}$ NMR $\left(400 \mathrm{MHz}, \mathrm{CD}_{3} \mathrm{CN}\right): \delta=6.08(\mathrm{~d}, J=4.0 \mathrm{~Hz}, 2 \mathrm{H}), 1.21(\mathrm{~d}, J=1.0 \mathrm{~Hz}, 18 \mathrm{H}) \mathrm{ppm}$.

${ }^{13} \mathrm{C}$ NMR (101 MHz, CD $\left.{ }_{3} \mathrm{CN}\right): \delta=121.5(\mathrm{~d}, J=7.1 \mathrm{~Hz}), 54.2(\mathrm{~d}, J=12.2 \mathrm{~Hz}), 29.8(\mathrm{~d}, J=8.9 \mathrm{~Hz}) \mathrm{ppm}$.

${ }^{31} \mathbf{P}$ NMR $\left(162 \mathrm{MHz}, \mathrm{CD}_{3} \mathrm{CN}\right): \delta=55.94 \mathrm{ppm}$.

HRMS (ESI): calcd. for $\left[\mathrm{C}_{10} \mathrm{H}_{20}{ }^{2} \mathrm{HN}_{2} \mathrm{P}\right]^{+},[\mathrm{M}]^{+}:$:201.1500; found: 201.1508 . 


\section{1,3-Di-tert-butyl-1,3-dihydro-2H-1,3,2-diazaphosphol-2-yl trifluoromethanesulfonate (DAP-OTf)}

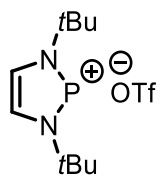

DAP-OTf

f) To a stirred solution of DAP-Br (1.00 g, $3.58 \mathrm{mmol}, 1.00$ equiv.) in dry and degassed THF (30 mL) was added trimethylsilyl trifluoromethanesulfonate $(647 \mu \mathrm{L}, 796 \mathrm{mg}, 3.58 \mathrm{mmol}, 1.00$ equiv.) at rt. The yellow solution turned immediately colorless and precipitation was observed. The mixture was stirred for $2 \mathrm{~h}$ and the precipitate was removed by filtration over celite. All volatiles were removed in vacuo to afford DAP-OTf (1.03 g, $2.95 \mathrm{mmol}$, $82 \%)$ as a white solid.

Analytical data of DAP-OTf:

${ }^{1} \mathbf{H}$ NMR $\left(400 \mathrm{MHz}, \mathrm{CDCl}_{3}\right): \delta=8.23(\mathrm{~s}, 2 \mathrm{H}), 1.80(\mathrm{~d}, J=1.9 \mathrm{~Hz}, 18 \mathrm{H}) \mathrm{ppm}$.

${ }^{13}$ C NMR (100 MHz, $\left.\mathrm{CDCl}_{3}\right): \delta=132.9(\mathrm{~d}, J=3.6 \mathrm{~Hz}), 62.8(\mathrm{~d}, J=7.6 \mathrm{~Hz}), 31.4(\mathrm{~d}, J=9.4 \mathrm{~Hz}) \mathrm{ppm}$.

${ }^{31} \mathbf{P}$ NMR (162 MHz, $\left.\mathrm{CDCl}_{3}\right): \delta=202.10 \mathrm{ppm}$.

All spectroscopic data in corresponded to the reported literature values. ${ }^{[10]}$

\section{1,3-Di-tert-butyl-2-iodo-2,3-dihydro-1H-1,3,2-diazaphosphole (DAP-I)}

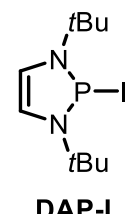

g) To a stirred suspension of (DAP) 2 (797 mg, $2.00 \mathrm{mmol}, 1.00$ equiv.) in MeCN (6 mL) was added iodobenzene ( $223 \mu \mathrm{L}, 408 \mathrm{mg}, 1.00 \mathrm{mmol}, 1.00$ equiv.) at rt. The mixture was stirred for $2 \mathrm{~h}$ and all volatiles were removed in vacuo. The bright yellow solid residue was washed with $n$-pentane and dried in vacuo to afford DAP-I (608 mg, $1.86 \mathrm{mmol}, 93 \%$ ) as a bright yellow solid.

Analytical data of DAP-I:

${ }^{1} \mathbf{H}$ NMR $\left(400 \mathrm{MHz}, \mathrm{CDCl}_{3}\right): \delta=7.33(\mathrm{~s}, 2 \mathrm{H}), 1.75(\mathrm{~d}, J=2.0 \mathrm{~Hz}, 19 \mathrm{H}) \mathrm{ppm}$.

${ }^{13} \mathrm{C} \mathrm{NMR}\left(100 \mathrm{MHz}, \mathrm{CDCl}_{3}\right): \delta=125.9(\mathrm{~d}, J=6.5 \mathrm{~Hz}), 60.1(\mathrm{~d}, J=7.1 \mathrm{~Hz}), 29.8(\mathrm{~d}, J=9.6 \mathrm{~Hz}) \mathrm{ppm}$.

${ }^{31} \mathbf{P}$ NMR $\left(162 \mathrm{MHz}, \mathrm{CDCl}_{3}\right): \delta=194.35 \mathrm{ppm}$.

${ }^{1} \mathrm{H}$ NMR $\left(400 \mathrm{MHz}, \mathrm{CD}_{3} \mathrm{CN}\right): \delta=7.84(\mathrm{~d}, J=3.3 \mathrm{~Hz}, 2 \mathrm{H}), 1.71(\mathrm{~d}, J=2.0 \mathrm{~Hz}, 20 \mathrm{H}) \mathrm{ppm}$.

${ }^{31} \mathbf{P}$ NMR (162 MHz, $\left.\mathrm{CD}_{3} \mathrm{CN}\right): \delta=199.31 \mathrm{ppm}$.

All spectroscopic data (acquired in $\mathrm{CDCl}_{3}$ ) corresponded to the reported literature values. ${ }^{[6]}$ 


\subsection{Substrate Synthesis}

\section{General Procedure C:}

Without exclusion of moisture or oxygen. To a stirred suspension of $\mathrm{K}_{2} \mathrm{CO}_{3}(2.00-3.00$ equiv.) and alcohol (1.00 equiv.) in DMF ( $0.5 \mathrm{M})$ was added alkyl halide $(1.1-1.3$ equiv.) at rt. The reaction was stirred for $16 \mathrm{~h}$ and diluted with $\mathrm{H}_{2} \mathrm{O}$ ( $2 \mathrm{~mL}$ per mmol alcohol) and aq. $\mathrm{NaOH}(2 \mathrm{M}, 2 \mathrm{~mL}$ per mmol alcohol). The mixture was extracted with EtOAc or $n$-pentane (3x) and the combined organic layers were washed with sat. aq. $\mathrm{Na}_{2} \mathrm{~S}_{2} \mathrm{O}_{3}$, followed by brine. After drying over $\mathrm{Na}_{2} \mathrm{SO}_{4}$, all volatiles were removed under reduced pressure and the residue was purified by flash chromatography $\left(\mathrm{SiO}_{2}, n\right.$-pentane:EtOAc).

\section{General Procedure D:}

A stirred suspension of amide (1.00 equiv.), $\mathrm{K}_{2} \mathrm{CO}_{3}$ (2.00 equiv.), $\mathrm{KI}$ (10 mol\%) and alkyl bromide (1.30 equiv.) in acetone $(0.5 \mathrm{M})$ was heated to $60{ }^{\circ} \mathrm{C}$. After $18 \mathrm{~h}$, the mixture was cooled to rt, diluted with $\mathrm{H}_{2} \mathrm{O}(10 \mathrm{~mL}$ per mmol amide $)$ and extracted with $\mathrm{Et}_{2} \mathrm{O}(3 \times 10 \mathrm{~mL}$ per mmol amide $)$. After drying the combined organic layers over $\mathrm{Na}_{2} \mathrm{SO}_{4}$, all volatiles were removed under reduced pressure and the residue was purified by flash chromatography $\left(\mathrm{SiO}_{2}, n\right.$-pentane:EtOAc).

\section{General Procedure E:}

To a stirred solution of $\mathrm{PPh}_{3}$ (3.00 equiv.) in dry THF (0.5 M) was added $\mathrm{CBr}_{4}$ (3.00 equiv.) at $0{ }^{\circ} \mathrm{C}$. The resulting mixture was stirred for $5 \mathrm{~min}$, followed by the addition of alcohol (1.00 equiv., dissolved in 1-2 $\mathrm{mL}$ dry THF). The reaction was allowed to reach $\mathrm{rt}$, stirred for $18 \mathrm{~h}$ and quenched by the addition of $\mathrm{H}_{2} \mathrm{O}$ ( $20 \mathrm{~mL}$ per mmol alcohol). The mixture was extracted with $\mathrm{CH}_{2} \mathrm{Cl}_{2}(3 \times 20 \mathrm{~mL}$ per mmol alcohol $)$, combined organic layers were dried over $\mathrm{MgSO}_{4}$ and all volatiles were removed under reduced pressure. Purification of the residue by flash chromatography $\left(\mathrm{SiO}_{2}, n\right.$-pentane:EtOAc) afforded the desired products. 


\section{1-Iodo-2-((3-methylbut-2-en-1-yl)oxy)benzene (1a)}

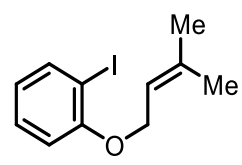

$1 \mathrm{a}$

Synthesized according to GP C from 2-iodophenol (1.10 g, $5.00 \mathrm{mmol}, 1.00$ equiv.), $\mathrm{K}_{2} \mathrm{CO}_{3}(2.07 \mathrm{~g}, 15.0 \mathrm{mmol}$, 3.00 equiv.) and 1-bromo-3-methylbut-2-ene ( $654 \mu \mathrm{L}, 820 \mathrm{mg}, 5.50 \mathrm{mmol}, 1.10$ equiv.). Purification by flash chromatography $\left(\mathrm{SiO}_{2}, n\right.$-pentane:EtOAc $\left.=40: 1\right)$ afforded $\mathbf{1 a}(1.25 \mathrm{~g}, 4.35 \mathrm{mmol}, 87 \%)$ as a colourless liquid.

Analytical data of $\mathbf{1 a}$ :

TLC $\left(\mathrm{SiO}_{2}\right): \mathrm{R}_{f}(n$-pentane: EtOAc $=40: 1)=0.43$

${ }^{1} \mathbf{H}$ NMR $\left(400 \mathrm{MHz}, \mathrm{CDCl}_{3}\right): \delta=7.77(\mathrm{dd}, J=7.8,1.6 \mathrm{~Hz}, 1 \mathrm{H}), 7.32-7.23(\mathrm{~m}, 1 \mathrm{H}), 6.83(\mathrm{dd}, J=8.2,1.4 \mathrm{~Hz}$, $1 \mathrm{H}), 6.70(\mathrm{td}, J=7.6,1.4 \mathrm{~Hz}, 1 \mathrm{H}), 5.54-5.49(\mathrm{~m}, 1 \mathrm{H}), 4.59(\mathrm{~d}, J=6.5 \mathrm{~Hz}, 2 \mathrm{H}), 1.80(\mathrm{~s}, 3 \mathrm{H}), 1.75(\mathrm{~s}, 3 \mathrm{H}) \mathrm{ppm}$. ${ }^{13}$ C NMR (101 MHz, $\left.\mathrm{CDCl}_{3}\right): \delta=157.6,139.6,138.1,129.5,122.6,119.7,112.8,87.1,66.4,25.9,18.5$ ppm. All spectroscopic data corresponded to the reported literature values. ${ }^{[6]}$

\section{1-Bromo-2-((3-methylbut-2-en-1-yl)oxy)benzene (2a)}

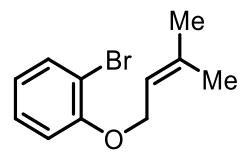

$2 a$

Synthesized according to GP C from 2-bromophenol ( $1.59 \mathrm{~mL}, 2.56 \mathrm{~g}, 15.0 \mathrm{mmol}, 1.11$ equiv.), $\mathrm{K}_{2} \mathrm{CO}_{3}(6.22 \mathrm{~g}$, $45.0 \mathrm{mmol}, 3.33$ equiv.) and 1-bromo-3-methylbut-2-ene (1.58 mL, $2.01 \mathrm{~g}, 13.5 \mathrm{mmol}, 1.00$ equiv.). Purification by flash chromatography $\left(\mathrm{SiO}_{2}, n\right.$-pentane:EtOAc $\left.=40: 1\right)$ afforded $\mathbf{2 a}(2.79 \mathrm{~g}, 11.6 \mathrm{mmol}, 86 \%)$ as a colourless liquid.

Analytical data of $\mathbf{2 a}$ :

TLC $\left(\mathrm{SiO}_{2}\right): \mathrm{R}_{f}(n$-pentane: EtOAc $=40: 1)=0.42$

${ }^{1} \mathbf{H}$ NMR $\left(400 \mathrm{MHz}, \mathrm{CDCl}_{3}\right): \delta=7.53(\mathrm{dd}, J=7.8,1.6 \mathrm{~Hz}, 1 \mathrm{H}), 7.29-7.20(\mathrm{~m}, 1 \mathrm{H}), 6.91(\mathrm{dd}, J=8.3,1.4 \mathrm{~Hz}$, $1 \mathrm{H}), 6.82(\mathrm{td}, J=7.6,1.4 \mathrm{~Hz}, 1 \mathrm{H}), 5.56-5.46(\mathrm{~m}, 1 \mathrm{H}), 4.60(\mathrm{~d}, J=6.5 \mathrm{~Hz}, 2 \mathrm{H}), 1.80(\mathrm{~s}, 3 \mathrm{H}), 1.75(\mathrm{~s}, 3 \mathrm{H}) \mathrm{ppm}$. ${ }^{13} \mathrm{C}$ NMR (101 MHz, $\left.\mathrm{CDCl}_{3}\right): \delta=155.4,138.2,133.5,128.5,121.9,119.6,113.9,112.6,66.3,26.0,18.5 \mathrm{ppm}$. All spectroscopic data corresponded to the reported literature values. ${ }^{[11]}$

\section{1-Chloro-2-((3-methylbut-2-en-1-yl)oxy)benzene (3a)}

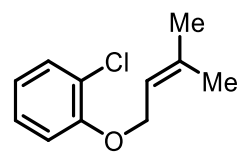

$3 a$

Synthesized according to GP C from 2-chlorophenol (3.36 $\mu \mathrm{L}, 424 \mathrm{mg}, 3.30 \mathrm{mmol}, 1.10$ equiv.), $\mathrm{K}_{2} \mathrm{CO}_{3}(1.24 \mathrm{~g}$, 9.00 mmol, 3.00 equiv.) and 1-bromo-3-methylbut-2-ene (352 $\mu \mathrm{L}, 447 \mathrm{mg}, 3.00 \mathrm{mmol}, 1.00$ equiv.). Purification by flash chromatography $\left(\mathrm{SiO}_{2}, n\right.$-pentane:EtOAc $\left.=40: 1\right)$ afforded $\mathbf{3 a}(506 \mathrm{mg}, 2.57 \mathrm{mmol}, 86 \%)$ as a colourless liquid. 
Analytical data of $\mathbf{3 a}$ :

TLC $\left(\mathrm{SiO}_{2}\right): \mathrm{R}_{f}(n$-pentane: EtOAc $=40: 1)=0.42$

${ }^{1} \mathbf{H}$ NMR $\left(400 \mathrm{MHz}, \mathrm{CDCl}_{3}\right): \delta=7.36(\mathrm{dd}, J=7.9,1.7 \mathrm{~Hz}, 1 \mathrm{H}), 7.24-7.15(\mathrm{~m}, 1 \mathrm{H}), 6.93(\mathrm{dd}, J=8.3,1.4 \mathrm{~Hz}, 1 \mathrm{H})$, $6.93-6.84(\mathrm{~m}, 1 \mathrm{H}), 5.56-5.47(\mathrm{~m}, 1 \mathrm{H}), 4.60(\mathrm{~d}, J=6.6 \mathrm{~Hz}, 2 \mathrm{H}), 1.79(\mathrm{~s}, 3 \mathrm{H}), 1.75(\mathrm{~s}, 3 \mathrm{H}) \mathrm{ppm}$.

${ }^{13} \mathrm{C}$ NMR $\left(101 \mathrm{MHz}, \mathrm{CDCl}_{3}\right): \delta=154.5,138.4,130.4,127.7,123.2,121.4,119.6,114.0,66.2,26.0,18.4 \mathrm{ppm}$.

All spectroscopic data corresponded to the reported literature values. ${ }^{[12]}$

\section{1-Bromo-4-methoxy-2-((3-methylbut-2-en-1-yl)oxy)benzene (2b)}

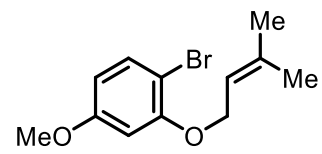

$2 \mathbf{b}$

Synthesized according to GP C from 2-bromo-5-methoxyphenol ( $374 \mu \mathrm{L}, 609 \mathrm{mg}$, $3.00 \mathrm{mmol}, 1.00$ equiv.), $\mathrm{K}_{2} \mathrm{CO}_{3}$

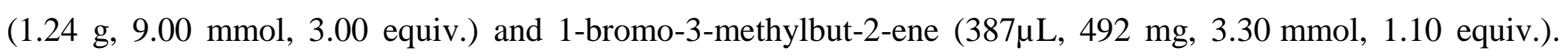
Purification by flash chromatography $\left(\mathrm{SiO}_{2}, n\right.$-pentane:EtOAc $\left.=20: 1\right)$ afforded $\mathbf{2 b}(650 \mathrm{mg}, 2.40 \mathrm{mmol}, 80 \%)$ as a colourless liquid.

Analytical data of $\mathbf{2 b}$ :

TLC $\left(\mathrm{SiO}_{2}\right): \mathrm{R}_{f}(n$-pentane:EtOAc $=20: 1)=0.48$

${ }^{1} \mathbf{H}$ NMR $\left(400 \mathrm{MHz}, \mathrm{CDCl}_{3}\right): \delta=7.11(\mathrm{~d}, J=3.0 \mathrm{~Hz}, 1 \mathrm{H}), 6.89-6.75(\mathrm{~m}, 2 \mathrm{H}), 5.54-5.45(\mathrm{~m}, 1 \mathrm{H}), 4.52(\mathrm{~d}, J=$ $7.0 \mathrm{~Hz}, 2 \mathrm{H}), 3.76(\mathrm{~s}, 3 \mathrm{H}), 1.78(\mathrm{~s}, 3 \mathrm{H}), 1.72(\mathrm{~s}, 3 \mathrm{H}) \mathrm{ppm}$.

${ }^{13}$ C NMR $\left(101 \mathrm{MHz}, \mathrm{CDCl}_{3}\right): \delta=154.3,149.8,138.2,119.9,118.9,115.7,113.8,113.3,67.3,56.0,26.0,18.4$ ppm.

IR (ATR): 2968 (w), 2935 (w), 1491 (s), 1440 (w) 1273 (m), 1212 (s), 1039 (s), 996 (w), 757 (w) cm-1.

HRMS (APPI/LTQ-Orbitrap): calcd. for $\left[\mathrm{C}_{12} \mathrm{H}_{15} \mathrm{BrO}_{2}+\mathrm{H}\right]^{+},[\mathrm{M}+\mathrm{H}]^{+}:$271.0328; found: 271.0325.

\section{2-Bromo-1-((3-methylbut-2-en-1-yl)oxy)-4-(trifluoromethyl)benzene (2c)}

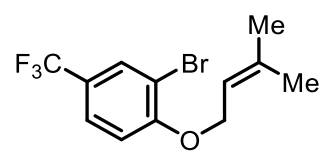

2c

Synthesized according to GP C from 2-bromo-4-(trifluoromethyl)phenol (723 mg, $3.00 \mathrm{mmol}, 1.00$ equiv.), $\mathrm{K}_{2} \mathrm{CO}_{3}$

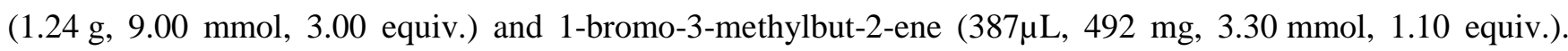
Purification by flash chromatography $\left(\mathrm{SiO}_{2}, n\right.$-pentane:EtOAc $\left.=40: 1\right)$ afforded $\mathbf{2 c}(649 \mathrm{mg}, 2.01 \mathrm{mmol}, 70 \%)$ as a colourless liquid.

Analytical data of $2 c$ :

TLC $\left(\mathrm{SiO}_{2}\right): \mathrm{R}_{f}(n$-pentane:EtOAc $=40: 1)=0.64$

${ }^{1} \mathbf{H}$ NMR $\left(400 \mathrm{MHz}, \mathrm{CDCl}_{3}\right): \delta=7.80(\mathrm{~d}, J=1.9 \mathrm{~Hz}, 1 \mathrm{H}), 7.55-7.47(\mathrm{~m}, 1 \mathrm{H}), 6.94(\mathrm{~d}, J=8.6 \mathrm{~Hz}, 1 \mathrm{H}), 5.53-$ $5.44(\mathrm{~m}, 1 \mathrm{H}), 4.65(\mathrm{~d}, J=6.5 \mathrm{~Hz}, 2 \mathrm{H}), 1.80(\mathrm{~s}, 3 \mathrm{H}), 1.76(\mathrm{~s}, 3 \mathrm{H}) \mathrm{ppm}$.

${ }^{13} \mathbf{C ~ N M R}\left(101 \mathrm{MHz}, \mathrm{CDCl}_{3}\right): \delta=157.9,139.2,130.7\left(\mathrm{q},{ }^{3} J_{\mathrm{FC}}=3.8 \mathrm{~Hz}\right), 125.9\left(\mathrm{q},{ }^{3} J_{\mathrm{FC}}=3.9 \mathrm{~Hz}\right), 123.8\left(\mathrm{q},{ }^{2} J_{\mathrm{FC}}=\right.$ $33.2 \mathrm{~Hz}), 123.7\left(\mathrm{q},{ }^{1} J_{\mathrm{FC}}=271.6 \mathrm{~Hz}\right), 118.7,112.9,112.5,66.5,26.0,18.5 \mathrm{ppm}$. 
${ }^{19}$ F NMR $\left(376 \mathrm{MHz}, \mathrm{CDCl}_{3}\right) \delta=-61.67 \mathrm{ppm}$.

IR (ATR): 2974 (w), 1607 (m), 1502 (m), 1322 (s), 1267 (s), 1117 (s), 1079 (s), 1047 (s), 980 (m), 813 (m), 686 (w), $618(\mathrm{w}) \mathrm{cm}^{-1}$.

HRMS (APPI/LTQ- Orbitrap): calcd. for $\left[\mathrm{C}_{12} \mathrm{H}_{12} \mathrm{BrF}_{3} \mathrm{O}\right]^{+},[\mathrm{M}]^{+}:$308.0018; found: 308.0015.

\section{2-Bromo-3-((3-methylbut-2-en-1-yl)oxy)phenol (2d)}

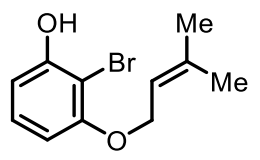

2d

Synthesized according to GP C from 2-bromobenzene-1,3-diol (1.13 g, $6.00 \mathrm{mmol}, 1.00$ equiv.), $\mathrm{K}_{2} \mathrm{CO}_{3}(2.49 \mathrm{~g}$, $18.0 \mathrm{mmol}, 3.00$ equiv.) and 1-bromo-3-methylbut-2-ene (1.04 mL, $1.34 \mathrm{~g}, 9.00 \mathrm{mmol}, 1.50$ equiv.). Purification by flash chromatography $\left(\mathrm{SiO}_{2}, n\right.$-pentane:EtOAc $\left.=20: 1-10: 1\right)$ afforded $\mathbf{2 d}(434 \mathrm{mg}, 1.69 \mathrm{mmol}, 56 \%)$ as a white solid.

Analytical data of $\mathbf{2 d}$ :

TLC $\left(\mathrm{SiO}_{2}\right): \mathrm{R}_{f}(n$-pentane:EtOAc $=10: 1)=0.37$

Mp: $34.3-36.7^{\circ} \mathrm{C}$.

${ }^{1} \mathbf{H}$ NMR $\left(400 \mathrm{MHz}, \mathrm{CDCl}_{3}\right): \delta=7.14(\mathrm{t}, J=8.3 \mathrm{~Hz}, 1 \mathrm{H}), 6.67(\mathrm{dd}, J=8.3,1.3 \mathrm{~Hz}, 1 \mathrm{H}), 6.48(\mathrm{dd}, J=8.3,1.3 \mathrm{~Hz}$, $1 \mathrm{H}), 5.62(\mathrm{~s}, 1 \mathrm{H}), 5.55-5.46(\mathrm{~m}, 1 \mathrm{H}), 4.58(\mathrm{~d}, J=6.5 \mathrm{~Hz}, 2 \mathrm{H}), 1.80(\mathrm{~s}, 3 \mathrm{H}), 1.75(\mathrm{~s}, 3 \mathrm{H}) \mathrm{ppm}$.

${ }^{13}$ C NMR (101 MHz, $\left.\mathrm{CDCl}_{3}\right): \delta=156.0,153.6,138.4,128.7,119.5,108.5,105.3,100.8,66.4,26.0,18.5$ ppm. IR (ATR): 3493 (br), 2931 (w), 2913 (w), 1592 (s), 1459 (s), 1266 (m), 1195 (s), 1053 (s), 766 (s) cm-

HRMS (APPI/LTQ-Orbitrap): calcd. for $\left[\mathrm{C}_{11} \mathrm{H}_{13} \mathrm{BrO}_{2}\right]^{+},[\mathrm{M}]^{+}:$256.0093; found: 256.0090 .

\section{2-Bromo-1,3-bis((3-methylbut-2-en-1-yl)oxy)benzene (2e)}

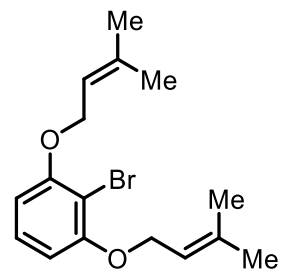

$\mathbf{2 e}$

The title compound was isolated during the synthesis of 2-Bromo-3-((3-methylbut-2-en-1-yl)oxy)phenol (2d) following GP C. Purification by flash chromatography $\left(\mathrm{SiO}_{2}, n\right.$-pentane:EtOAc $\left.=20: 1-10: 1\right)$ afforded $\mathbf{2 e}(527 \mathrm{mg}$, $1.62 \mathrm{mmol}, 54 \%)$ as a white solid.

Analytical data of $2 \boldsymbol{e}$ :

TLC $\left(\mathrm{SiO}_{2}\right): \mathrm{R}_{f}(n$-pentane:EtOAc $=10: 1)=0.70$

Mp: $86.2-89.3{ }^{\circ} \mathrm{C}$.

${ }^{1} \mathbf{H}$ NMR $\left(400 \mathrm{MHz}, \mathrm{CDCl}_{3}\right): \delta=7.16(\mathrm{t}, J=8.3 \mathrm{~Hz}, 1 \mathrm{H}), 6.56(\mathrm{~d}, J=8.3 \mathrm{~Hz}, 2 \mathrm{H}), 5.57-5.44(\mathrm{~m}, 2 \mathrm{H}), 4.59$ (dt, $J=6.5,1.0 \mathrm{~Hz}, 4 \mathrm{H}), 1.78(\mathrm{~s}, 6 \mathrm{H}), 1.74(\mathrm{~s}, 6 \mathrm{H}) \mathrm{ppm}$.

${ }^{13} \mathrm{C}$ NMR (101 MHz, $\mathrm{CDCl}_{3}$ ): $\delta=156.8$ (2C), 138.0 (2C), 128.0, 119.8 (2C), 106.4 (2C), 102.6, 66.5 (2C), 26.0 (2C), 18.5 (2C) ppm. 
IR (ATR): 2973 (w), 2913 (w), 1589 (m), 1458 (s), 1382 (m), 1252 (m), 1234 (m), 1074 (s), 1036 (m), 760 (m) cm ${ }^{-1}$. HRMS (APPI/LTQ-Orbitrap): calcd. for $\left[\mathrm{C}_{16} \mathrm{H}_{22} \mathrm{BrO}_{2}+\mathrm{H}\right]^{+},[\mathrm{M}+\mathrm{H}]^{+}: 325.0798$; found: 325.0790 .

\section{3-Bromo-4-((3-methylbut-2-en-1-yl)oxy)benzoic acid (2f)}

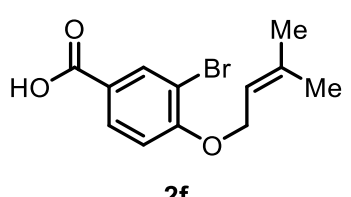

$2 f$

To a stirred solution of methyl 3-bromo-4-((3-methylbut-2-en-1-yl)oxy)benzoate (2g) (299 mg, $1.00 \mathrm{mmol}, 1.00$ equiv.) in THF/MeOH (1:1, $4 \mathrm{~mL}$ ) was added $\mathrm{NaOH}$ (44 mg, 1.10 mmol, 1.10 equiv.) dissolved in $\mathrm{H}_{2} \mathrm{O}(1 \mathrm{~mL})$. The resulting mixture was heated to $60^{\circ} \mathrm{C}$ for $19 \mathrm{~h}$. After the reaction was cooled to rt, aq. $\mathrm{HCl}(1 \mathrm{M}, 10 \mathrm{~mL})$ were added, and the resulting mixture was extracted with EtOAc $(3 \times 15 \mathrm{~mL})$. Combined organic layers were dried over $\mathrm{Na}_{2} \mathrm{SO} 4$ and all volatiles were removed under reduced pressure to afford the title compound $2 \mathbf{f}(276 \mathrm{mg}, 968 \mu \mathrm{mol}$, $97 \%)$ as a white solid.

Analytical data of $2 f$ :

Mp: $155.8-157.9^{\circ} \mathrm{C}$.

${ }^{1}$ H NMR $\left(400 \mathrm{MHz}, \mathrm{CDCl}_{3}\right): \delta=11.98(\mathrm{br} \mathrm{s}, 1 \mathrm{H}), 8.30(\mathrm{~d}, J=2.1 \mathrm{~Hz}, 1 \mathrm{H}), 8.02(\mathrm{dd}, J=8.7,2.1 \mathrm{~Hz}, 1 \mathrm{H}), 6.93(\mathrm{~d}$, $J=8.7 \mathrm{~Hz}, 1 \mathrm{H}), 5.54-5.46(\mathrm{~m}, 1 \mathrm{H}), 4.68(\mathrm{~d}, J=6.6 \mathrm{~Hz}, 2 \mathrm{H}), 1.81(\mathrm{~s}, 3 \mathrm{H}), 1.77$ (s, 3H) ppm.

${ }^{13} \mathbf{C}$ NMR $\left(101 \mathrm{MHz}, \mathrm{CDCl}_{3}\right): \delta=170.6,159.8,139.3,135.7,131.3,122.6,118.7,112.5,112.2,66.5,26.0$, $18.6 \mathrm{ppm}$.

IR (ATR): 2913 (br), 1678 (s), 1596 (s), 1427 (m), 1318 (m), 1274 (s), 1046 (w), 802 (m) cm².

HRMS (ESI): calcd. for $\left[\mathrm{C}_{12} \mathrm{H}_{13} \mathrm{BrO}_{3}-\mathrm{H}\right]^{-},[\mathrm{M}-\mathrm{H}]^{-:}$:282.9975; found: 282.9972.

Methyl 3-bromo-4-((3-methylbut-2-en-1-yl)oxy)benzoate (2g)

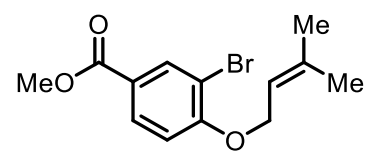

$2 \mathrm{~g}$

Synthesized according to GP C from 3-bromo-4-hydroxybenzoate (693 mg, $3.00 \mathrm{mmol}, 1.00$ equiv.), $\mathrm{K}_{2} \mathrm{CO}_{3}$

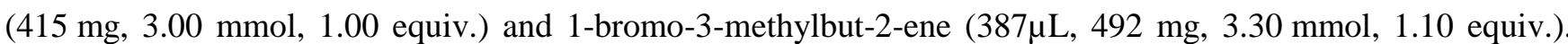
Purification by flash chromatography $\left(\mathrm{SiO}_{2}, n\right.$-pentane:EtOAc $\left.=20: 1\right)$ afforded $\mathbf{2 g}(639 \mathrm{mg}, 2.14 \mathrm{mmol}, 71 \%)$ as a white solid.

Analytical data of $\mathbf{2 g}$ :

TLC $\left(\mathrm{SiO}_{2}\right): \mathrm{R}_{f}(n$-pentane:EtOAc $=20: 1)=0.27$

${ }^{1} \mathbf{H}$ NMR $\left(400 \mathrm{MHz}, \mathrm{CDCl}_{3}\right): \delta=8.23(\mathrm{~d}, J=2.1 \mathrm{~Hz}, 1 \mathrm{H}), 7.95(\mathrm{dd}, J=8.6,2.1 \mathrm{~Hz}, 1 \mathrm{H}), 6.90(\mathrm{~d}, J=8.7 \mathrm{~Hz}, 1 \mathrm{H})$, $5.54-5.44(\mathrm{~m}, 1 \mathrm{H}), 4.66(\mathrm{~d}, J=6.5 \mathrm{~Hz}, 2 \mathrm{H}), 3.89(\mathrm{~s}, 3 \mathrm{H}), 1.80(\mathrm{~s}, 3 \mathrm{H}), 1.76(\mathrm{~s}, 3 \mathrm{H}) \mathrm{ppm}$.

${ }^{13} \mathbf{C}$ NMR $\left(101 \mathrm{MHz}, \mathrm{CDCl}_{3}\right): \delta=165.9,159.0,139.0,135.0,130.6,123.6,118.8,112.4,112.1,66.4,52.3,26.0$, $18.5 \mathrm{ppm}$.

All spectroscopic data corresponded to the reported literature values. ${ }^{[12]}$ 


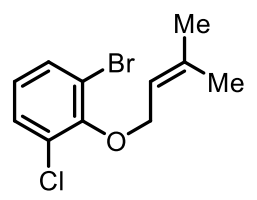

$\mathbf{2 h}$

Synthesized according to GP C from 2-bromo-6-chlorophenol (622 mg, $3.00 \mathrm{mmol}, 1.00$ equiv.), $\mathrm{K}_{2} \mathrm{CO}_{3}(1.24 \mathrm{~g}$, $9.00 \mathrm{mmol}, 3.00$ equiv.) and 1-bromo-3-methylbut-2-ene (387 $\mu \mathrm{L}, 492 \mathrm{mg}, 3.30 \mathrm{mmol}, 1.10$ equiv.). Purification by flash chromatography $\left(\mathrm{SiO}_{2}, n\right.$-pentane:EtOAc $\left.=40: 1\right)$ afforded $\mathbf{2 h}(103 \mathrm{mg}, 281 \mu \mathrm{mol}, 84 \%)$ as a colourless oil.

Analytical data of $\mathbf{2 h}$ :

TLC $\left(\mathrm{SiO}_{2}\right): \mathrm{R}_{f}(n$-pentane:EtOAc $=40: 1)=0.64$

${ }^{1} \mathbf{H}$ NMR (400 MHz, $\left.\mathrm{CDCl}_{3}\right): \delta=7.45(\mathrm{dd}, J=8.1,1.5 \mathrm{~Hz}, 1 \mathrm{H}), 7.33(\mathrm{dd}, J=8.0,1.5 \mathrm{~Hz}, 1 \mathrm{H}), 6.91(\mathrm{t}, J=8.0 \mathrm{~Hz}$, $1 \mathrm{H}), 5.69-5.59(\mathrm{~m}, 1 \mathrm{H}), 4.55(\mathrm{dt}, J=7.4,0.8 \mathrm{~Hz}, 2 \mathrm{H}), 1.80(\mathrm{~s}, 3 \mathrm{H}), 1.75$ (s, 3H) ppm.

${ }^{13} \mathbf{C}$ NMR $\left(101 \mathrm{MHz}, \mathrm{CDCl}_{3}\right): \delta=152.5,139.9,132.0,129.8,129.8,125.7,119.5,119.3,70.1,26.0,18.2$ ppm. IR (ATR): 2930 (br), 1674 (w), 1559 (w), 1435 (s), 1379 (m), 1240 (s), 1068 (w), 946 (s), 766 (s), 752 (s) cm . HRMS (ESI): calcd. for $\left[\mathrm{C}_{11} \mathrm{H}_{12} \mathrm{BrClO}+\mathrm{Na}\right]^{+},[\mathrm{M}+\mathrm{Na}]^{+}: 296.9652$; found: 296.9663.

2-Bromo-3-((3-methylbut-2-en-1-yl)oxy)pyridine (2i)

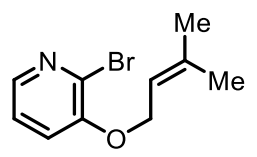

$\mathbf{2 i}$

Synthesized according to GP C from 2-bromopyridin-3-ol (522 mg, $3.00 \mathrm{mmol}, 1.00$ equiv.), $\mathrm{K}_{2} \mathrm{CO}_{3}(1.24 \mathrm{~g}, 9.00$

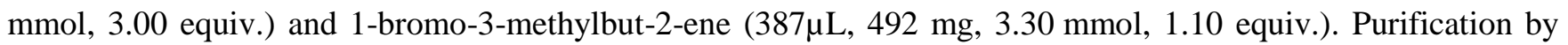
flash chromatography $\left(\mathrm{SiO}_{2}, n\right.$-pentane:EtOAc $\left.=10: 1\right)$ afforded $\mathbf{2 i}(580 \mathrm{mg}, 2.40 \mathrm{mmol}, 80 \%)$ as a colourless liquid. Analytical data of $2 i$ :

TLC $\left(\mathrm{SiO}_{2}\right): \mathrm{R}_{f}(n$-pentane:EtOAc $=10: 1)=0.26$

${ }^{1} \mathbf{H}$ NMR $\left(400 \mathrm{MHz}, \mathrm{CDCl}_{3}\right): \delta=7.96(\mathrm{dd}, J=4.7,1.5 \mathrm{~Hz}, 1 \mathrm{H}), 7.22-7.14(\mathrm{~m}, 1 \mathrm{H}), 7.12(\mathrm{dd}, J=8.1,1.6 \mathrm{~Hz}$, $1 \mathrm{H}), 5.51-5.40(\mathrm{~m}, 1 \mathrm{H}), 4.61(\mathrm{~d}, J=6.6 \mathrm{~Hz}, 2 \mathrm{H}), 1.79(\mathrm{~s}, 3 \mathrm{H}), 1.75(\mathrm{~s}, 3 \mathrm{H}) \mathrm{ppm}$.

${ }^{13}$ C NMR (101 MHz, $\left.\mathrm{CDCl}_{3}\right): \delta=152.4,141.3,139.3,133.4,123.4,120.3,118.7,66.3,25.9,18.5 \mathrm{ppm}$.

All spectroscopic data corresponded to the reported literature values. ${ }^{[12]}$

\section{1-Bromo-3-iodo-2-((3-methylbut-2-en-1-yl)oxy)benzene (1j)}

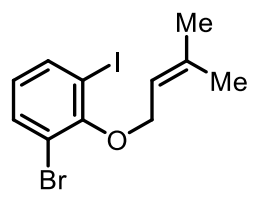

$1 \mathrm{j}$

To a stirred solution of 2-bromo-6-iodophenol (100 mg, $335 \mu \mathrm{mol}, 1.00$ equiv.), triphenylphosphane (87.7 mg, 335 $\mu \mathrm{mol}, 1.00$ equiv. ) and 3-methylbut-2-en-1-ol (40.8 $\mu \mathrm{L}, 34.6 \mathrm{mg}, 401 \mu \mathrm{mol}, 1.20$ equiv.) in THF ( $2 \mathrm{~mL})$ was added 
DIAD (65.7 $\mu \mathrm{L}, 67.6 \mathrm{mg}, 335 \mu \mathrm{mol}, 1.00$ equiv.) at $\mathrm{rt}$. The resulting mixture was heated to $80{ }^{\circ} \mathrm{C}$ for $18 \mathrm{~h}$. The reaction was cooled to rt and all volatiles were removed under reduced pressure. Purification of the residue by flash chromatography $\left(\mathrm{SiO}_{2}, n\right.$-pentane:EtOAc $\left.=40: 1\right)$ afforded $\mathbf{1 j}(103 \mathrm{mg}, 281 \mu \mathrm{mol}, 84 \%)$ as a colourless oil.

Analytical data of $\mathbf{1 j}$ :

TLC $\left(\mathrm{SiO}_{2}\right): \mathrm{R}_{f}(n$-pentane:EtOAc $=40: 1)=0.34$

${ }^{1} \mathbf{H}$ NMR (400 MHz, CDCl $): \delta=7.73(\mathrm{dd}, J=7.9,1.5 \mathrm{~Hz}, 1 \mathrm{H}), 7.53(\mathrm{dd}, J=7.9,1.5 \mathrm{~Hz}, 1 \mathrm{H}), 6.70(\mathrm{t}, J=7.9 \mathrm{~Hz}$, $1 \mathrm{H}), 5.73-5.63(\mathrm{~m}, 1 \mathrm{H}), 4.53(\mathrm{~d}, J=7.3 \mathrm{~Hz}, 2 \mathrm{H}), 1.82(\mathrm{~s}, 3 \mathrm{H}), 1.79$ (s, 3H) ppm.

${ }^{13}$ C NMR (101 MHz, $\left.\mathrm{CDCl}_{3}\right): \delta=155.7,139.8,138.8,133.9,127.1,119.6,117.5,93.4,70.2,26.0,18.5$ ppm.

IR (ATR): 2930 (br), 1550 (w), 1429 (s), 1378 (m), 1234 (m), 1068 (w), 945 (s), 766 (s), 703 (s) cm-1.

HRMS (APPI/LTQ-Orbitrap): calcd. for $\left[\mathrm{C}_{11} \mathrm{H}_{12} \mathrm{BrIO}^{+}\right.$, [M] ${ }^{+}: 365.9111$; found: 365.9106.

\section{(E)-1-Bromo-2-(cinnamyloxy)benzene (2k)}

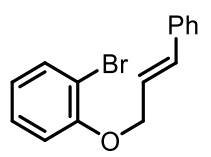

2k

Synthesized according to GP C from 2-bromophenol ( $531 \mu \mathrm{L}, 865 \mathrm{mg}, 5.00 \mathrm{mmol}, 1.00$ equiv.), $\mathrm{K}_{2} \mathrm{CO}_{3}(2.07 \mathrm{~g}$, $15.0 \mathrm{mmol}, 3.00$ equiv.) and [(E)-3-bromoprop-1-enyl]benzene (1.08 g, $5.50 \mathrm{mmol}, 1.10$ equiv.). Purification by flash chromatography $\left(\mathrm{SiO}_{2}, n\right.$-pentane:EtOAc $\left.=40: 1-20: 1\right)$ afforded $2 \mathbf{k}(2.79 \mathrm{~g}, 11.6 \mathrm{mmol}, 86 \%)$ as a colourless oil.

Analytical data of $\mathbf{2 k}$ :

TLC $\left(\mathrm{SiO}_{2}\right): \mathrm{R}_{f}(n$-pentane: EtOAc $=40: 1)=0.35$

${ }^{1} \mathbf{H}$ NMR (400 MHz, CDCl $): \delta=7.56(\mathrm{dd}, J=7.9,1.6 \mathrm{~Hz}, 1 \mathrm{H}), 7.45-7.40(\mathrm{~m}, 2 \mathrm{H}), 7.36-7.31(\mathrm{~m}, 2 \mathrm{H}), 7.29-$ $7.23(\mathrm{~m}, 2 \mathrm{H}), 6.96(\mathrm{dd}, J=8.2,1.4 \mathrm{~Hz}, 1 \mathrm{H}), 6.85(\mathrm{td}, J=7.6,1.4 \mathrm{~Hz}, 1 \mathrm{H}), 6.79(\mathrm{dt}, J=15.9,1.7 \mathrm{~Hz}, 1 \mathrm{H}), 6.43(\mathrm{dt}$, $J=15.9,5.6 \mathrm{~Hz}, 1 \mathrm{H}), 4.79(\mathrm{dd}, J=5.6,1.6 \mathrm{~Hz}, 2 \mathrm{H}) \mathrm{ppm}$.

${ }^{13} \mathbf{C}$ NMR (101 MHz, $\left.\mathrm{CDCl}_{3}\right): \delta=155.2,136.5,133.6,133.2,128.8,128.6,128.1,126.8,124.1,122.3,114.0,112.6$, $69.9 \mathrm{ppm}$.

All spectroscopic data corresponded to the reported literature values. ${ }^{[13]}$

1-Bromo-2-((2-methylallyl)oxy)benzene (2l)

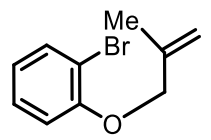

21

Synthesized according to GP C from 2-bromophenol (318 $\mu \mathrm{L}, 519 \mathrm{mg}, 3.00 \mathrm{mmol}, 1.00$ equiv.), $\mathrm{K}_{2} \mathrm{CO}_{3}(1.24 \mathrm{~g}$, 9.00 mmol, 3.00 equiv.) and 3-bromo-2-methylprop-1-ene (332 $\mu \mathrm{L}, 446 \mathrm{mg}, 3.30 \mathrm{mmol}, 1.10$ equiv.). Purification by flash chromatography $\left(\mathrm{SiO}_{2}, n\right.$-pentane:EtOAc $\left.=40: 1\right)$ afforded $2 \mathbf{l}(676 \mathrm{mg}, 2.98 \mathrm{mmol}, 99 \%)$ as a colourless liquid.

Analytical data of $\mathbf{2 l}$ :

TLC $\left(\mathrm{SiO}_{2}\right): \mathrm{R}_{f}(n$-pentane:EtOAc $=40: 1)=0.42$ 
${ }^{1} \mathbf{H}$ NMR (400 MHz, $\left.\mathrm{CDCl}_{3}\right): \delta=7.54(\mathrm{~d}, \mathrm{~J}=7.9,1 \mathrm{H}), 7.28-7.19(\mathrm{~m}, 1 \mathrm{H}), 6.89(\mathrm{~d}, \mathrm{~J}=8.3,1 \mathrm{H}), 6.83(\mathrm{t}, \mathrm{J}=7.6$, $1 \mathrm{H}), 5.20-5.14(\mathrm{~m}, 1 \mathrm{H}), 5.05-4.96(\mathrm{~m}, 1 \mathrm{H}), 4.50(\mathrm{~s}, 2 \mathrm{H}), 1.86(\mathrm{~s}, 3 \mathrm{H}) \mathrm{ppm}$.

${ }^{13}$ C NMR (101 MHz, $\left.\mathrm{CDCl}_{3}\right): \delta=155.1,140.4,133.5,128.5,122.0,113.6,113.0,112.4,72.6,19.5$ ppm.

All spectroscopic data corresponded to the reported literature values. ${ }^{[14]}$

\section{Cyclohex-1-en-1-ylmethanol (SI-2)}

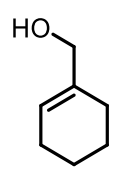

SI-2

To a stirred solution of $\mathrm{LiAlH}_{4}(1.14 \mathrm{~g}, 12.5 \mathrm{~mL}, 30.0 \mathrm{mmol}, 2.40 \mathrm{M}$ in THF, 3.00 equiv.) was added a solution of cyclohexene-1-carboxylic acid (1.26 g, $10.0 \mathrm{mmol}, 1.00$ equiv.) in $\mathrm{Et}_{2} \mathrm{O}(25 \mathrm{~mL})$ dropwise at $0{ }^{\circ} \mathrm{C}$. The mixture was stirred for $3 \mathrm{~h}$ and quenched by the careful addition of $\mathrm{H}_{2} \mathrm{O}(10 \mathrm{~mL})$. Aq. $\mathrm{NaOH}(2 \mathrm{M}, 30 \mathrm{~mL})$, EtOAc (30 $\mathrm{mL})$ and $\mathrm{CH}_{2} \mathrm{Cl}_{2}(50 \mathrm{~mL})$ was added. Filtration over a pad of celite, allowed to separate the layers. The organic layer was dried over $\mathrm{Na}_{2} \mathrm{SO}_{4}$, volatiles were removed under reduced pressure and the residue was purified by flash chromatography $\left(\mathrm{SiO}_{2}, n\right.$-pentane:EtOAc $\left.=10: 1-3: 1\right)$ to afford $\mathbf{S I - 2}(780 \mathrm{mg}, 6.95 \mathrm{mmol}, 70 \%)$ as a faint yellow liquid.

Analytical data of SI-2:

TLC $\left(\mathrm{SiO}_{2}\right): \mathrm{R}_{f}(n$-pentane: EtOAc $=10: 1)=0.20$

${ }^{1} \mathbf{H}$ NMR (400 MHz, CDCl $): \delta=5.71-5.64(\mathrm{~m}, 1 \mathrm{H}), 3.97$ (s, 2H), $2.07-1.96(\mathrm{~m}, 4 \mathrm{H}), 1.71-1.52$ (m, 4H) ppm.

${ }^{13} \mathbf{C}$ NMR (101 MHz, $\left.\mathrm{CDCl}_{3}\right): \delta=137.7,123.2,67.8,25.7,25.1,22.7,22.6 \mathrm{ppm}$.

All spectroscopic data corresponded to the reported literature values. ${ }^{[15]}$

\section{1-Bromo-2-(cyclohex-1-en-1-ylmethoxy)benzene (2m)}

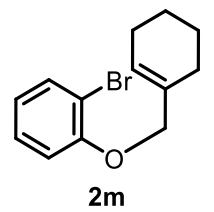

To a stirred solution of cyclohexen-1-ylmethanol (SI-2) (123 mg, $1.10 \mathrm{mmol}, 1.10$ equiv.uiv) in dry $\mathrm{Et}_{2} \mathrm{O}$ (5 mL) was added $\mathrm{PBr}_{3}\left(104 \mu \mathrm{L}, 298 \mathrm{mg}, 1.10 \mathrm{mmol}, 1.10\right.$ equiv.) at $0{ }^{\circ} \mathrm{C}$. The reaction mixture was stirred for $1 \mathrm{~h}$ and carefully quenched by the addition of sat. aq. $\mathrm{NH}_{4} \mathrm{Cl}(5 \mathrm{~mL})$. The layers were separated and the organic layer, containing the corresponding bromide, was directly used in GP C with 2-bromophenol (116 $\mu \mathrm{L}, 173 \mathrm{mg}, 1.00 \mathrm{mmol}$, 1.00 equiv.) and $\mathrm{K}_{2} \mathrm{CO}_{3}\left(415 \mathrm{mg}, 3.00 \mathrm{mmol}, 3.00\right.$ equiv.). Purification by flash chromatography $\left(\mathrm{SiO}_{2}\right.$, $n$-pentane:EtOAc $=40: 1)$ afforded $\mathbf{2 m}(92 \mathrm{mg}, 344 \mu \mathrm{mol}, 34 \%)$ as a colourless oil.

Analytical data of $2 \mathrm{~m}$ :

TLC $\left(\mathrm{SiO}_{2}\right): \mathrm{R}_{f}(n$-pentane: EtOAc $=40: 1)=0.50$

${ }^{1} \mathbf{H}$ NMR $\left(400 \mathrm{MHz}, \mathrm{CDCl}_{3}\right): \delta=7.53(\mathrm{dd}, J=7.8,1.6 \mathrm{~Hz}, 1 \mathrm{H}), 7.23(\mathrm{ddd}, J=8.2,7.4,1.6 \mathrm{~Hz}, 1 \mathrm{H}), 6.90(\mathrm{dd}, J=$ $8.3,1.4 \mathrm{~Hz}, 1 \mathrm{H}), 6.82(\mathrm{td}, J=7.6,1.4 \mathrm{~Hz}, 1 \mathrm{H}), 5.89-5.82(\mathrm{~m}, 1 \mathrm{H}), 4.44(\mathrm{~s}, 2 \mathrm{H}), 2.16-2.03(\mathrm{~m}, 4 \mathrm{H}), 1.76-1.65$ (m, 2H), $1.65-1.57(\mathrm{~m}, 2 \mathrm{H}) \mathrm{ppm}$. 
${ }^{13} \mathbf{C}$ NMR (101 MHz, $\left.\mathrm{CDCl}_{3}\right): \delta=155.4,133.4,133.4,128.4,125.6,121.9,113.8,112.5,73.7,25.8,25.1,22.5$, $22.4 \mathrm{ppm}$.

IR (ATR): 2926 (m), 1585 (w), 1478 (s), 1442 (m), 1291 (s), 1247 (s), 1049 (m), 1030 (s), 999 (m), 745 (s) cmHRMS (APPI/LTQ-Orbitrap): calcd. for $\left[\mathrm{C}_{13} \mathrm{H}_{15} \mathrm{BrO}+\mathrm{H}\right]^{+},[\mathrm{M}+\mathrm{H}]^{+}:$267.0379; found: 267.0377.

(E)-1-Bromo-2-((3,7-dimethylocta-2,6-dien-1-yl)oxy)benzene (2n)

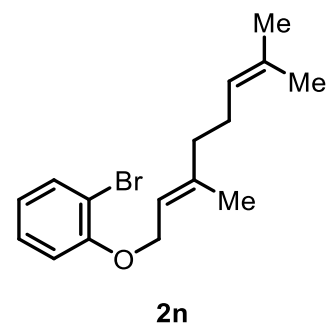

To a stirred solution of (3E)-4,8-dimethylnona-3,7-dien-1-ol ( $989 \mu \mathrm{L}, 879 \mathrm{mg}, 5.23 \mathrm{mmol}, 1.10$ equiv.) in dry Et2O $(13 \mathrm{~mL})$ was added tribromophosphane $\left(1.48 \mathrm{~g}, 519 \mu \mathrm{L}, 5.46 \mathrm{mmol}, 1.15\right.$ equiv.) at $0{ }^{\circ} \mathrm{C}$ and exclusion of light. The mixture was stirred for $15 \mathrm{~min}$ and quenched by the careful addition of sat. aq. $\mathrm{NH}_{4} \mathrm{Cl}(13 \mathrm{~mL})$. Layers were separated and the organic layer, containing the corresponding alkyl bromide, was directly used in GP C to synthesize $2 \mathrm{n}$ from 2-bromophenol (548 $\mu \mathrm{L}, 822 \mathrm{mg}$, $4.75 \mathrm{mmol}, 1.00$ equiv.) and $\mathrm{K}_{2} \mathrm{CO}_{3}$ (2.63 g, $19.0 \mathrm{mmol}, 4.00$ equiv.). Purification by flash chromatography $\left(\mathrm{SiO}_{2}, n\right.$-pentane:EtOAc $\left.=1: 0-40: 1\right)$ afforded the title compound (985 mg, $3.19 \mathrm{mmol}, 67 \%$ ) as a colourless liquid.

Analytical data of $\mathbf{2 n}$ :

TLC $\left(\mathrm{SiO}_{2}\right): \mathrm{R}_{f}(n$-pentane:EtOAc $=40: 1)=0.43$

${ }^{1} \mathbf{H}$ NMR $\left(400 \mathrm{MHz}, \mathrm{CDCl}_{3}\right): \delta=7.53(\mathrm{dd}, J=7.8,1.6 \mathrm{~Hz}, 1 \mathrm{H}), 7.28-7.19(\mathrm{~m}, 1 \mathrm{H}), 6.90(\mathrm{dd}, \mathrm{J}=8.2,1.4 \mathrm{~Hz}$, $1 \mathrm{H}), 6.82(\mathrm{td}, \mathrm{J}=7.6,1.4 \mathrm{~Hz}, 1 \mathrm{H}), 5.54-5.47(\mathrm{~m}, 1 \mathrm{H}), 5.13-5.05(\mathrm{~m}, 1 \mathrm{H}), 4.63(\mathrm{~d}, \mathrm{~J}=6.4 \mathrm{~Hz}, 2 \mathrm{H}), 2.18-2.03$ (m, 4H), 1.74 (s, 3H), 1.67 (s, 3H), 1.61 (s, 3H) ppm.

${ }^{13} \mathrm{C}$ NMR (101 MHz, $\left.\mathrm{CDCl}_{3}\right): \delta=155.4,141.3,133.5,132.0,128.4,123.9,121.9,119.5,114.0,112.6,66.4,39.7$, 26.4, 25.8, 17.9, $16.9 \mathrm{ppm}$.

IR (ATR): 2917 (m), 1586 (w), 1477 (s), 1442 (m), 1276 (m), 1235 (m), 1031 (m), 994 (m), 746 (s) cm ${ }^{-1}$.

HRMS (ESI): calcd. for $\left[\mathrm{C}_{16} \mathrm{H}_{21} \mathrm{BrO}+\mathrm{Na}\right]^{+},[\mathrm{M}+\mathrm{Na}]^{+}: 331.0668$; found: 331.0667 .

\section{1-Bromo-2-((4-methylpent-3-en-1-yl)oxy)benzene (2o)}

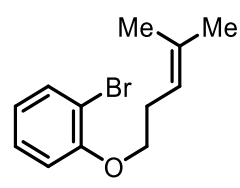

20

Synthesized according to GP C from 2-bromophenol ( $265 \mu \mathrm{L}, 433 \mathrm{mg}, 2.50 \mathrm{mmol}, 1.49$ equiv.), $\mathrm{K}_{2} \mathrm{CO}_{3}(1.04 \mathrm{~g}$, $7.5 \mathrm{mmol}, 4.47$ equiv.) and 5-bromo-2-methylpent-2-ene ( $255 \mu \mathrm{L}, 274 \mathrm{mg}, 1.68 \mathrm{mmol}, 1.00$ equiv.). Purification by flash chromatography $\left(\mathrm{SiO}_{2}, n\right.$-pentane:EtOAc $\left.=1: 0-40: 1\right)$ afforded $20(256 \mathrm{mg}, 1.00 \mathrm{mmol}, 60 \%)$ as a colourless liquid.

Analytical data of 2o:

TLC $\left(\mathrm{SiO}_{2}\right): \mathrm{R}_{f}(n$-pentane: EtOAc $=40: 1)=0.50$ 
${ }^{1} \mathbf{H}$ NMR (400 MHz, $\left.\mathrm{CDCl}_{3}\right): \delta=7.53(\mathrm{dd}, J=7.9,1.6 \mathrm{~Hz}, 1 \mathrm{H}), 7.28-7.19(\mathrm{~m}, 1 \mathrm{H}), 6.88(\mathrm{dd}, J=8.3,1.4 \mathrm{~Hz}$, $1 \mathrm{H}), 6.81(\mathrm{td}, J=7.6,1.4 \mathrm{~Hz}, 1 \mathrm{H}), 5.29-5.19(\mathrm{~m}, 1 \mathrm{H}), 3.99(\mathrm{t}, J=7.1 \mathrm{~Hz}, 2 \mathrm{H}), 2.59-2.49(\mathrm{~m}, 2 \mathrm{H}), 1.74(\mathrm{~s}, 3 \mathrm{H})$, $1.68(\mathrm{~s}, 3 \mathrm{H})$.

${ }^{13} \mathbf{C}$ NMR $\left(101 \mathrm{MHz}, \mathrm{CDCl}_{3}\right): \delta=155.5,134.8,133.5,128.5,121.8,119.4,113.4,112.4,77.5,77.2,76.8,68.9$, 28.3, 26.0, $18.0 \mathrm{ppm}$.

IR (ATR): 2989 (w), 2916 (w), 2877 (w), 1586 (w), 1481 (m), 1442 (m), 1293 (m), 1247 (s), 1051 (s), 1030 (s), $745(\mathrm{~s}) \mathrm{cm}^{-1}$.

HRMS (ESI): calcd. for $\left[\mathrm{C}_{12} \mathrm{H}_{15} \mathrm{BrO}+\mathrm{Na}\right]^{+},[\mathrm{M}+\mathrm{Na}]^{+}: 277.0198$; found: 277.0199.

\section{$N$-(2-Bromophenyl)acetamide (SI-3)}

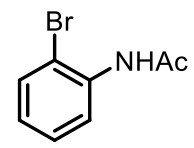

SI-3

To a stirred solution of 2-bromoaniline (1.72 g, $10.0 \mathrm{mmol}, 1.00$ equiv.uiv) and DMAP (61.1 mg, $500 \mu \mathrm{mol}$, $5 \mathrm{~mol} \%)$ in $\mathrm{CHCl}_{3}(40 \mathrm{~mL})$ was $\mathrm{Ac}_{2} \mathrm{O}(945 \mu \mathrm{L} 1.02 \mathrm{~g}, 10.0 \mathrm{mmol}, 1.00$ equiv.uiv) dropwise. The mixture was refluxed for $3 \mathrm{~h}$, cooled down to rt and washed with $\mathrm{H}_{2} \mathrm{O}(40 \mathrm{~mL})$. The organic layer was dried over $\mathrm{MgSO}_{4}$ and all volatiles were removed under reduced pressure. Purification of the residue by flash chromatography $\left(\mathrm{SiO}_{2}\right.$, $n$-pentane:EtOAc $=3: 1)$ afforded $\mathbf{S I - 3}(1.91 \mathrm{~g}, 8.93 \mathrm{mmol}, 89 \%)$ as a white solid.

Analytical data of SI-3:

TLC $\left(\mathrm{SiO}_{2}\right): \mathrm{R}_{f}(n$-pentane:EtOAc $=3: 1)=0.43$

${ }^{1} \mathbf{H}$ NMR $\left(400 \mathrm{MHz}, \mathrm{CDCl}_{3}\right): \delta=8.33(\mathrm{~d}, J=8.2 \mathrm{~Hz}, 1 \mathrm{H}), 7.60$ (br s, $\left.1 \mathrm{H}\right), 7.53(\mathrm{~d}, J=8.1 \mathrm{~Hz}, 1 \mathrm{H}), 7.34-7.28(\mathrm{~m}$, $1 \mathrm{H}), 7.03-6.94(\mathrm{~m}, 1 \mathrm{H}), 2.24(\mathrm{~s}, 3 \mathrm{H}) \mathrm{ppm}$.

${ }^{13}$ C NMR (101 MHz, $\left.\mathrm{CDCl}_{3}\right): \delta=168.4,135.8,132.3,128.5,125.3,122.1,113.3,25.0 \mathrm{ppm}$.

All spectroscopic data corresponded to the reported literature values. ${ }^{[16]}$

\section{$N$-(2-Bromophenyl)- $N$-(3-methylbut-2-en-1-yl)acetamide (2p)}

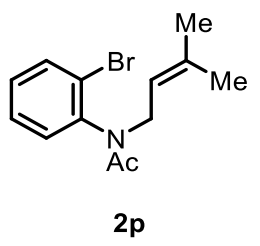

To a stirred solution of $N$-(2-bromophenyl)acetamide (SI-3) (642 mg, $3.00 \mathrm{mmol}, 1.00$ equiv.) in THF ( $9 \mathrm{~mL}$ ) was added sodium hydride (120 mg, $3.00 \mathrm{mmol}, 1.00$ equiv.) (60 wt $\%$ in mineral oil) at $0{ }^{\circ} \mathrm{C}$. The mixture was stirred for $5 \mathrm{~min}$ and 1-bromo-3-methylbut-2-ene ( $387 \mu \mathrm{L}, 492 \mathrm{mg}, 3.30 \mathrm{mmol}, 1.10$ equiv.) was added. The reaction was allowed to reach $\mathrm{rt}$, stirred for further $5 \mathrm{~h}$ and quenched by the addition of $\mathrm{H}_{2} \mathrm{O}(10 \mathrm{~mL})$. The resulting mixture was extracted with EtOAc $(3 \times 15 \mathrm{~mL})$. Combined organic layers were dried over $\mathrm{Na}_{2} \mathrm{SO}_{4}$ and all volatiles were removed under reduced pressure. Purification of the residue by flash chromatography $\left(\mathrm{SiO}_{2}, n\right.$-pentane:EtOAc $\left.=3: 1\right)$ afforded $2 \mathbf{p}$ (778 $\mathrm{mg}, 2.76 \mathrm{mmol}, 92 \%$ ) as a colourless liquid.

Analytical data of $2 p$ :

TLC $\left(\mathrm{SiO}_{2}\right): \mathrm{R}_{f}(n$-pentane:EtOAc $=3: 1)=0.50$ 
${ }^{1} \mathbf{H}$ NMR $\left(400 \mathrm{MHz}, \mathrm{CDCl}_{3}\right): \delta=7.66(\mathrm{dd}, J=7.9,1.5 \mathrm{~Hz}, 1 \mathrm{H}), 7.34(\mathrm{td}, J=7.6,1.5 \mathrm{~Hz}, 1 \mathrm{H}), 7.22(\mathrm{td}, J=7.8$, $1.7 \mathrm{~Hz}, 1 \mathrm{H}), 7.17$ (dd, $J=7.7,1.7 \mathrm{~Hz}, 1 \mathrm{H}), 5.28-5.18(\mathrm{~m}, 1 \mathrm{H}), 4.73-4.63(\mathrm{~m}, 1 \mathrm{H}), 3.81(\mathrm{dd}, J=14.5,8.2 \mathrm{~Hz}$, 1H), 1.79 (s, 3H), 1.64 (s, 3H), 1.39 (s, 3H) ppm.

${ }^{13}$ C NMR (101 MHz, $\left.\mathrm{CDCl}_{3}\right): \delta=170.0,141.6,137.0,133.8,131.3,129.7,128.5,124.2,118.9,45.3,25.8,22.6$, $17.6 \mathrm{ppm}$.

All spectroscopic data corresponded to the reported literature values. ${ }^{[12]}$

\section{2-Bromo- $N$-(3-methylbut-2-en-1-yl)aniline (SI-4)}

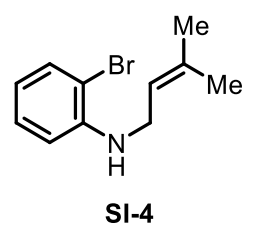

To a stirred solution of 2-bromoaniline (516 mg, $3.00 \mathrm{mmol}, 1.00$ equiv.) in dry THF (10 mL) was added LDA $\left(1.50 \mathrm{~mL}, 3.00 \mathrm{mmol}, 2.00 \mathrm{M}\right.$ in THF, 1.00 equiv.) at $-78{ }^{\circ} \mathrm{C}$. After $5 \mathrm{~min}, 1$-bromo-3-methylbut-2-ene (312 $\mu \mathrm{L}$, $402 \mathrm{mg}, 2.70 \mathrm{mmol}, 0.900$ equiv.) was added dropwise. The reaction was allowed to warm to $\mathrm{rt}$ and quenched by the addition of aq. sat. $\mathrm{NH}_{4} \mathrm{Cl}(10 \mathrm{~mL})$. Layers were separated and the aqueous layer was extracted with $n$-pentane $(3 \times 20 \mathrm{~mL})$. Combined organic layers were dried over $\mathrm{Na}_{2} \mathrm{SO}_{4}$ and all volatiles were removed under reduced pressure. Purification by flash chromatography $\left(\mathrm{SiO}_{2}, n\right.$-pentane:EtOAc = 40:1) afforded SI-4 $(581 \mathrm{mg}, 2.42 \mathrm{mmol}$, $81 \%)$ as a colourless oil.

Analytical data of SI-4:

TLC $\left(\mathrm{SiO}_{2}\right): \mathrm{R}_{f}(n$-pentane:EtOAc $=40: 1)=0.54$

${ }^{1} \mathbf{H}$ NMR (400 MHz, $\left.\mathrm{CDCl}_{3}\right): \delta=7.42(\mathrm{dd}, \mathrm{J}=7.8,1.5 \mathrm{~Hz}, 1 \mathrm{H}), 7.21-7.15(\mathrm{~m}, 1 \mathrm{H}), 6.63(\mathrm{dd}, \mathrm{J}=8.2,1.5 \mathrm{~Hz}, 1 \mathrm{H})$, 6.57 (td, J = 7.6, $1.5 \mathrm{~Hz}, 1 \mathrm{H}), 5.39-5.30(\mathrm{~m}, 1 \mathrm{H}), 4.24$ (br s, 1H), $3.77-3.71(\mathrm{~m}, 2 \mathrm{H}), 1.78$ (s, 3H), 1.74 (s, 3H) ppm.

${ }^{13} \mathbf{C}$ NMR (101 MHz, $\left.\mathrm{CDCl}_{3}\right): \delta=145.2,136.3,132.5,128.6,121.2,117.7,111.5,109.8,42.0,25.9,18.2$ ppm. All spectroscopic data corresponded to the reported literature values. ${ }^{[17]}$

2-Bromo- $N, N$-bis(3-methylbut-2-en-1-yl)aniline (2q)

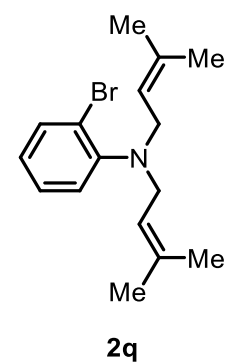

Synthesized according to GP C from 2-bromo- $N$-(3-methylbut-2-en-1-yl)aniline (SI-4) (240 mg, 1.00 mmol, 1.00 equiv.), $\mathrm{K}_{2} \mathrm{CO}_{3}$ (276 mg, $2.00 \mathrm{mmol}, 2.00$ equiv.) and 1-bromo-3-methylbut-2-ene (127 $\mu \mathrm{L}, 164 \mathrm{mg}, 1.10 \mathrm{mmol}$, 1.10 equiv.). Purification by flash chromatography $\left(\mathrm{SiO}_{2}, n\right.$-pentane:EtOAc $\left.=40: 1\right)$ afforded $\mathbf{2 q}(239 \mathrm{mg}, 775 \mu \mathrm{mol}$, $78 \%$ ) as a colourless oil.

Analytical data of $2 q$ : 
TLC $\left(\mathrm{SiO}_{2}\right): \mathrm{R}_{f}(n$-pentane:EtOAc $=40: 1)=0.54$

${ }^{1} \mathbf{H}$ NMR (400 MHz, $\left.\mathrm{CDCl}_{3}\right): \delta=7.55(\mathrm{dd}, J=7.9,1.5 \mathrm{~Hz}, 1 \mathrm{H}), 7.21(\mathrm{ddd}, J=8.4,7.3,1.6 \mathrm{~Hz}, 1 \mathrm{H}), 6.98(\mathrm{dd}, J=$ $8.0,1.6 \mathrm{~Hz}, 1 \mathrm{H}), 6.87(\mathrm{td}, J=7.6,1.6 \mathrm{~Hz}, 1 \mathrm{H}), 5.25-5.16(\mathrm{~m}, 2 \mathrm{H}), 3.63(\mathrm{~d}, J=7.5 \mathrm{~Hz}, 4 \mathrm{H}), 1.68(\mathrm{~s}, 6 \mathrm{H}), 1.60$ (s, $6 \mathrm{H}) \mathrm{ppm}$.

${ }^{13} \mathrm{C}$ NMR $\left(101 \mathrm{MHz}, \mathrm{CDCl}_{3}\right): \delta=149.8,134.8$ (2C), 133.8, 127.5, 124.0, 123.9, 121.5 (2C), 121.3, 50.4 (2C), 25.9 (2C), 18.1 (2C) ppm.

IR (ATR): 2969 (m), 2926 (m), 2812 (m), 1583 (w), 1473 (s), 1376 (m), 1226 (w), 1089 (m), 1026 (s), 753 (s) cm ${ }^{-1}$. HRMS (ESI): calcd. for $\left[\mathrm{C}_{16} \mathrm{H}_{22} \mathrm{BrN}+\mathrm{H}\right]^{+},[\mathrm{M}+\mathrm{H}]^{+}$: 308.1008; found: 308.1017.

\section{2,6-Dibromo- $N, N$-bis(3-methylbut-2-en-1-yl)aniline (2r)}

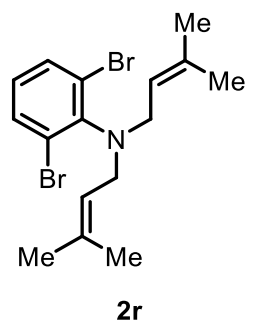

Synthesized according to GP C from 2,6-dibromoanilline (753 mg, $3.00 \mathrm{mmol}, 1.00$ equiv.), $\mathrm{K}_{2} \mathrm{CO}_{3}(2.07 \mathrm{~g}$, $15.0 \mathrm{mmol}, 5.00$ equiv.) and 3-bromo-2-methylprop-1-ene ( $809 \mu \mathrm{L}, 1.04 \mathrm{~g}, 6.30 \mathrm{mmol}, 2.10$ equiv.). Purification by flash chromatography $\left(\mathrm{SiO}_{2}, n\right.$-pentane:EtOAc $\left.=1: 0-20: 1\right)$ afforded $2 \mathbf{r}(391 \mathrm{mg}, 1.01 \mathrm{mmol}, 34 \%)$ as a colourless liquid.

Analytical data of $2 r$ :

TLC $\left(\mathrm{SiO}_{2}\right): \mathrm{R}_{f}(n$-pentane:EtOAc $=40: 1)=0.89$

${ }^{1} \mathbf{H}$ NMR $\left(400 \mathrm{MHz}, \mathrm{CDCl}_{3}\right): \delta=7.49(\mathrm{~d}, J=8.0 \mathrm{~Hz}, 2 \mathrm{H}), 6.81(\mathrm{t}, J=7.9 \mathrm{~Hz}, 1 \mathrm{H}), 5.34-5.25(\mathrm{~m}, 2 \mathrm{H}), 3.74(\mathrm{~d}, J$ $=6.9,4 \mathrm{H}), 1.66(\mathrm{~s}, 6 \mathrm{H}), 1.59(\mathrm{~s}, 6 \mathrm{H}) \mathrm{ppm}$.

${ }^{13}$ C NMR (101 MHz, $\mathrm{CDCl}_{3}$ ): $\delta=147.6,134.1$ (2C), 132.9 (2C), 127.7 (2C), 127.2, 122.8 (2C), 49.9 (2C), 26.0 (2C), 17.9 (2C) ppm.

IR (ATR): 2969 (w), 2911 (m), 1672 (w), 1544 (m), 1424 (s), 1375 (m), 1189 (w), 1133 (w), 925 (w), 843 (w), $769(\mathrm{~s}), 715(\mathrm{~s}) \mathrm{cm}^{-1}$.

HRMS (ESI): calcd. for $\left[\mathrm{C}_{16} \mathrm{H}_{21} \mathrm{Br}_{2} \mathrm{~N}+\mathrm{H}\right]^{+},[\mathrm{M}+\mathrm{H}]^{+}:$386.0114; found: 386.0120 .

3-Iodo-2-((3-methylbut-2-en-1-yl)oxy)tetrahydro-2H-pyran (1s)

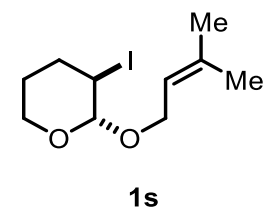

Synthesized according to a literature known procedure. ${ }^{[18]}$ To a stirred solution of 3-methylbut-2-en-1-ol (1.02 mL, $861 \mathrm{mg}, 10.0 \mathrm{mmol}, 1.00$ equiv.) and 3,4-dihydro-2H-pyran (998 $\mu \mathrm{L}, 925 \mathrm{mg}, 11.0 \mathrm{mmol}, 1.10$ equiv.) in dry $\mathrm{CH}_{2} \mathrm{Cl}_{2}(10 \mathrm{~mL})$ was added 1-iodopyrrolidine-2,5-dione (2.25 g, $10.0 \mathrm{mmol}, 1.00$ equiv.) at $-30{ }^{\circ} \mathrm{C}$. The reaction was stirred for $3 \mathrm{~h}$, diluted with $\mathrm{CH}_{2} \mathrm{Cl}_{2}(20 \mathrm{~mL})$, washed with $\mathrm{H}_{2} \mathrm{O}(20 \mathrm{~mL})$, aq. $\mathrm{Na}_{2} \mathrm{~S}_{2} \mathrm{O}_{3}(10 \%, 20 \mathrm{~mL})$, brine 
$(20 \mathrm{~mL})$ and dried over $\mathrm{Na}_{2} \mathrm{SO}_{4}$. Volatiles were removed under reduced pressure and the residue was purified by flash chromatography $\left(\mathrm{SiO}_{2}, n\right.$-pentane:EtOAc $\left.=10: 1\right)$ to afford $\mathbf{1 s}(2.68 \mathrm{~g}, 9.03 \mathrm{mmol}, 90 \%)$ as a colourless oil. Analytical data of 1 :

TLC $\left(\mathrm{SiO}_{2}\right): \mathrm{R}_{f}(n$-pentane: EtOAc $=10: 1)=0.58$

${ }^{1} \mathbf{H}$ NMR $\left(400 \mathrm{MHz}, \mathrm{CDCl}_{3}\right): \delta=5.41-5.32(\mathrm{~m}, 1 \mathrm{H}), 4.65(\mathrm{~d}, J=5.4 \mathrm{~Hz}, 1 \mathrm{H}), 4.22(\mathrm{dd}, J=11.6,6.6 \mathrm{~Hz}, 1 \mathrm{H})$, $4.14-4.02(\mathrm{~m}, 2 \mathrm{H}), 4.04-3.94(\mathrm{~m}, 1 \mathrm{H}), 3.58(\mathrm{ddd}, J=11.2,7.4,3.5 \mathrm{~Hz}, 1 \mathrm{H}), 2.42-2.33(\mathrm{~m}, 1 \mathrm{H}), 2.06-1.97$ (m, 1H), $1.80-1.72(\mathrm{~m}, 1 \mathrm{H}), 1.76(\mathrm{~s}, 3 \mathrm{H}), 1.69(\mathrm{~s}, 3 \mathrm{H}), 1.63-1.52(\mathrm{~m}, 1 \mathrm{H}) \mathrm{ppm}$.

${ }^{13}$ C NMR (101 MHz, $\left.\mathrm{CDCl}_{3}\right): \delta=138.2,120.2,101.5,64.7,63.6,33.0,29.8,26.0,25.7,18.2 \mathrm{ppm}$.

All spectroscopic data corresponded to the reported literature values. ${ }^{[18]}$

\section{3-Bromo-2-((3-methylbut-2-en-1-yl)oxy)tetrahydro-2H-pyran (2s)}

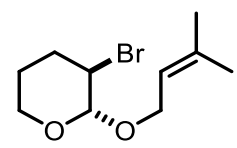

2s

To a stirred solution of 3-methylbut-2-en-1-ol (508 $\mu \mathrm{L}, 431 \mathrm{mg}, 5.00 \mathrm{mmol}, 1.00$ equiv.) and 3,4-dihydro-2H-pyran (499 $\mu \mathrm{L}, 463 \mathrm{mg}, 5.50 \mathrm{mmol}, 1.10$ equiv.) in $\mathrm{CH}_{2} \mathrm{Cl}_{2}(5 \mathrm{~mL}$ ) was added 1-bromopyrrolidine-2,5-dione (890 mg, $5.00 \mathrm{mmol}, 1.00$ equiv.) at $0{ }^{\circ} \mathrm{C}$. The mixture was stirred for $22 \mathrm{~h}$ at rt, followed by the addition of $\mathrm{CH}_{2} \mathrm{Cl}_{2}(10 \mathrm{~mL})$. The organic layer was washed with $\mathrm{H}_{2} \mathrm{O}(15 \mathrm{~mL})$, aq. $\mathrm{Na}_{2} \mathrm{~S}_{2} \mathrm{O}_{3}(10 \%, 20 \mathrm{~mL})$, brine $(20 \mathrm{~mL})$ and dried over $\mathrm{Na}_{2} \mathrm{SO}_{4}$. Volatiles were removed under reduced pressure and the residue was purified by flash chromatography $\left(\mathrm{SiO}_{2}\right.$, $n$-pentane:EtOAc $=10: 1)$ to afford $2 \mathrm{~s}(905 \mathrm{mg}, 3.63 \mathrm{mmol}, 73 \%)$ as a colourless oil.

Analytical data of $2 \mathrm{~s}$ :

TLC $\left(\mathrm{SiO}_{2}\right): \mathrm{R}_{f}(n$-pentane: EtOAc $=10: 1)=0.70$

${ }^{1} \mathbf{H}$ NMR $\left(400 \mathrm{MHz}, \mathrm{CDCl}_{3}\right): \delta=5.40-5.31(\mathrm{~m}, 1 \mathrm{H}), 4.63(\mathrm{~d}, J=4.4 \mathrm{~Hz}, 1 \mathrm{H}), 4.28-4.17(\mathrm{~m}, 2 \mathrm{H}), 4.07(\mathrm{dd}, J=$ 11.8, 7.6 Hz, 1H), $3.98(\mathrm{dt}, \mathrm{J}=6.6,4.3 \mathrm{~Hz}, 1 \mathrm{H}), 3.98-3.88(\mathrm{~m}, 1 \mathrm{H}), 3.63-3.53(\mathrm{~m}, 1 \mathrm{H}), 2.45-2.34(\mathrm{~m}, 1 \mathrm{H})$, $2.02-1.86(\mathrm{~m}, 2 \mathrm{H}), 1.76(\mathrm{~s}, 3 \mathrm{H}), 1.69(\mathrm{~s}, 3 \mathrm{H}), 1.58-1.46(\mathrm{~m}, 1 \mathrm{H}) \mathrm{ppm}$.

${ }^{13}$ C NMR (101 MHz, $\left.\mathrm{CDCl}_{3}\right): \delta=138.3,120.2,100.1,64.5,62.6,49.8,30.3,26.0,23.5,18.2 \mathrm{ppm}$.

All spectroscopic data corresponded to the reported literature values. ${ }^{[19]}$

\section{(5-Hydroxypentyl)triphenylphosphonium bromide (SI-5)}

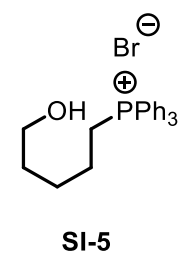

Prepared according to the literature procedure. ${ }^{[20]} \mathrm{A}$ stirred suspension of $\mathrm{PPh}_{3}$ (4.46 g, $17.0 \mathrm{mmol}, 1.00$ equiv.), 5bromopentan-1-ol (2.84 g, $17.0 \mathrm{mmol}, 1.00$ equiv.) and $\mathrm{K}_{2} \mathrm{CO}_{3}$ (2.35 g, $17.0 \mathrm{mmol}, 1.00$ equiv.) in $\mathrm{MeCN}(15 \mathrm{~mL})$ was heated to reflux for $4 \mathrm{~h}$. The reaction mixture was cooled to rt, filtered, and treated with $\mathrm{Et}_{2} \mathrm{O}(150 \mathrm{~mL})$ upon which a white gum precipitated. The solvents were decanted off and the remaining residue was washed with $\mathrm{Et}_{2} \mathrm{O}$ $(50 \mathrm{~mL})$. The gum was dried in vacuo to afford SI-5 (2.55 g, $5.95 \mathrm{mmol}, 35 \%)$ as a white solid. 
Analytical data of SI-5:

${ }^{1} \mathbf{H}$ NMR (400 MHz, $\left.\mathrm{CDCl}_{3}\right): \delta=7.87-7.67$ (m, 15H), $3.77-3.68(\mathrm{~m}, 4 \mathrm{H}), 3.15$ (br s, 1H), $1.81-1.63$ (m, 6H) ppm.

${ }^{13}$ C NMR (101 MHz, $\left.\mathrm{CDCl}_{3}\right): \delta=135.2,135.1,130.7,130.6,119.0,61.8,31.1,27.2,27.0,23.1,22.6$ ppm.

All spectroscopic data corresponded to the reported literature values. ${ }^{[21]}$

(E/Z)-6-Phenylhex-5-en-1-ol (SI-6)

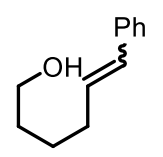

SI-6

Prepared according to the literature procedure. ${ }^{[22]}$ To a stirred suspension of (5-hydroxypentyl) triphenylphosphonium bromide (SI-5) $(2.15 \mathrm{~g}, 5.00 \mathrm{mmol}, 1.00$ equiv.) in dry THF (7.5 mL) was added $n$ BuLi (6.25 mL, $1.60 \mathrm{M}$ in $n$-hexane, $641 \mathrm{mg}, 10.0 \mathrm{mmol}, 2.00$ equiv.) at $0{ }^{\circ} \mathrm{C}$. A red coloured suspension was obtained, to which a solution of benzaldehyde ( $531 \mu \mathrm{L}, 557 \mathrm{mg}, 5.25 \mathrm{mmol}, 1.05$ equiv.) in dry THF (4 mL) was added dropwise. After $5 \mathrm{~min}$ the red colour vanished and an ivory, thick suspension was obtained. The mixture stirred for $4 \mathrm{~h}$ at $\mathrm{rt}$ and was poured into a mixture of $\mathrm{H}_{2} \mathrm{O}(25 \mathrm{~mL})$ and EtOAc $(25 \mathrm{~mL})$. The layers were separated, and the aqueous layer was extracted with EtOAc $(1 \times 10 \mathrm{~mL})$. Combined organic layers were washed with brine $(1 \times 15$ $\mathrm{mL}$ ) and dried over $\mathrm{Na} 2 \mathrm{SO} 4$. All volatiles were removed under reduced pressure and purification by flash chromatography $\left(\mathrm{SiO}_{2}, n\right.$-pentane:EtOAc $\left.=3: 1\right)$ afforded $\mathbf{S I - 6}(415 \mathrm{mg}, 2.36 \mathrm{mmol}, 47 \%, E: Z=79: 21)$ as a faint yellow oil.

Analytical data of SI-6:

TLC $\left(\mathrm{SiO}_{2}\right): \mathrm{R}_{f}(n$-pentane:EtOAc $=3: 1)=0.45$

${ }^{1} \mathbf{H}$ NMR (400 MHz, $\left.\mathrm{CDCl}_{3}\right)$ : (E)-isomer: $\delta=7.39-7.17(\mathrm{~m}, 5 \mathrm{H}), 6.47-6.36(\mathrm{~m}, 1 \mathrm{H}), 6.22(\mathrm{dt}, J=15.8,6.9 \mathrm{~Hz}$, $1 \mathrm{H}), 3.68(\mathrm{t}, J=6.4 \mathrm{~Hz}, 2 \mathrm{H}), 2.26(\mathrm{qd}, J=7.1,1.4 \mathrm{~Hz}, 2 \mathrm{H}), 1.70-1.49(\mathrm{~m}, 4 \mathrm{H}), 1.33$ (br s, $1 \mathrm{H})$; $(Z)$-isomer: $\delta=$ $7.39-7.17(\mathrm{~m}, 5 \mathrm{H}), 6.47-6.36(\mathrm{~m}, 1 \mathrm{H}), 5.66(\mathrm{dt}, \mathrm{J}=11.7,7.2 \mathrm{~Hz}, 1 \mathrm{H}), 3.63(\mathrm{t}, \mathrm{J}=6.4 \mathrm{~Hz}, 2 \mathrm{H}), 2.37(\mathrm{qd}, \mathrm{J}=7.2$, $1.8 \mathrm{~Hz}, 2 \mathrm{H}), 1.70-1.49(\mathrm{~m}, 4 \mathrm{H}), 1.33(\mathrm{~s}, 1 \mathrm{H}) \mathrm{ppm}$.

${ }^{13}$ C NMR (101 MHz, $\left.\mathrm{CDCl}_{3}\right): \delta=137.9,137.8,132.8,130.7,130.3,129.3,128.9,128.6,127.1,126.7,126.1,63.0$, $62.9,32.9,32.5,32.4,28.6,26.2,25.6 \mathrm{ppm}$.

All spectroscopic data corresponded to the reported literature values. ${ }^{[22,23]}$

(E/Z)-(6-Bromohex-1-en-1-yl)benzene (2t)

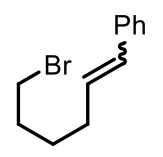

$2 \mathbf{t}$

Prepared according to the literature procedure. ${ }^{[22]}$ A solution of $\mathrm{PPh}_{3}(628 \mathrm{mg}, 2.39 \mathrm{mmol}, 1.05$ equiv.) in dry $\mathrm{CH}_{2} \mathrm{Cl}_{2}$ (3 mL) was slowly added to a solution of (E/Z)-6-phenylhex-5-en-1-ol (402 mg, $2.28 \mathrm{mmol}$, 1.00 equiv.) and $\mathrm{CBr}_{4}$ (832 mg, $2.51 \mathrm{mmol}, 1.10$ equiv.) in dry $\mathrm{CH}_{2} \mathrm{Cl}_{2}(11 \mathrm{~mL})$ at rt. The reaction mixture was stirred for $22 \mathrm{~h}$ and quenched by the addition of $\mathrm{H}_{2} \mathrm{O}(5 \mathrm{~mL})$. The resulting mixture was extracted with $\mathrm{CH}_{2} \mathrm{Cl}_{2}(3 \times 10 \mathrm{~mL})$, 
combined organic layers were dried over $\mathrm{MgSO}_{4}$ and all volatiles were removed under reduced pressure. Purification by flash chromatography $\left(\mathrm{SiO}_{2}\right.$, petroleum ether) afforded $\mathbf{2 t}(419 \mathrm{mg}, 1.75 \mathrm{mmol}, 77 \%, E: Z=78: 22)$ as a colourless liquid.

Analytical data of $2 t$ :

TLC $\left(\mathrm{SiO}_{2}\right): \mathrm{R}_{f}$ (petroleum ether) $=0.21$

${ }^{1}$ H NMR (400 MHz, $\mathrm{CDCl}_{3}$ ): (E)-isomer: $\delta=7.37-7.17(\mathrm{~m}, 5 \mathrm{H}), 6.45-6.36(\mathrm{~m}, 1 \mathrm{H}), 6.20(\mathrm{dt}, J=15.8,6.9 \mathrm{~Hz}$, 1H), $3.44(\mathrm{t}, J=6.8 \mathrm{~Hz}, 2 \mathrm{H}), 2.26(\mathrm{qd}, J=7.3,1.4 \mathrm{~Hz}, 2 \mathrm{H}), 2.00-1.85(\mathrm{~m}, 2 \mathrm{H}), 1.72-1.57(\mathrm{~m}, 2 \mathrm{H})$; (Z)-isomer: $\delta=7.38-7.16(\mathrm{~m}, 5 \mathrm{H}), 6.46(\mathrm{~d}, J=11.7 \mathrm{~Hz}, 1 \mathrm{H}), 5.65(\mathrm{dt}, J=11.7,7.2 \mathrm{~Hz}, 1 \mathrm{H}), 3.39(\mathrm{t}, J=6.8 \mathrm{~Hz}, 2 \mathrm{H}), 2.37$ (qd, $J=7.4,1.9 \mathrm{~Hz}, 2 \mathrm{H}), 2.00-1.85(\mathrm{~m}, 2 \mathrm{H}), 1.71-1.56(\mathrm{~m}, 2 \mathrm{H}) \mathrm{ppm}$.

${ }^{13} \mathbf{C}$ NMR (101 MHz, $\left.\mathrm{CDCl}_{3}\right): \delta=137.8,132.2,130.6,130.1,129.6,128.9,128.7,128.3,127.1,126.7,126.1$, $33.9,33.8,32.4,32.4,32.2,28.5,28.0,27.8 \mathrm{ppm}$.

All spectroscopic data corresponded to the reported literature values. ${ }^{[22]}$

\section{(E)-(3-(2-Bromoethoxy)prop-1-en-1-yl)benzene (2u)}

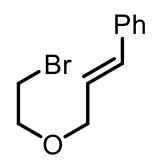

$2 \mathbf{u}$

Prepared according to the literature procedure. ${ }^{[24]}$ To a stirred solution of 2-bromoethanol ( $355 \mu \mathrm{L}, 625 \mathrm{mg}, 5.00$ mmol, 1.00 equiv. $)$ in dry THF $(2.5 \mathrm{~mL})$ was added $\mathrm{ZnEt}_{2}(2.50 \mathrm{~mL}, 1.00 \mathrm{M}$ in THF, $309 \mathrm{mg}, 2.50 \mathrm{mmol}, 0.500$ equiv.) dropwise at rt. The mixture was stirred for $15 \mathrm{~min}$, and [(E)-3-phenylprop-2-enyl] acetate (881 $\mathrm{mg}, 5.00$ mmol, 1.00 equiv.) and $\mathrm{Pd}\left(\mathrm{PPh}_{3}\right)_{4}(289 \mathrm{mg}, 250 \mu \mathrm{mol}, 5 \mathrm{~mol} \%)$ were added. The resulting mixture was stirred for $3 \mathrm{~h}$ and all volatiles were removed under reduced pressure. Purification by flash chromatography $\left(\mathrm{SiO}_{2}\right.$, $n$-pentane:EtOAc = 20:1) afforded $\mathbf{2 u}(692 \mathrm{mg}, 2.87 \mathrm{mmol}, 57 \%)$ as a colourless liquid.

Analytical data of $\mathbf{2 u}$ :

TLC $\left(\mathrm{SiO}_{2}\right): \mathrm{R}_{f}(n$-pentane:EtOAc $=20: 1)=0.57$

${ }^{1} \mathbf{H}$ NMR $\left(400 \mathrm{MHz}, \mathrm{CDCl}_{3}\right): \delta=7.42-7.38(\mathrm{~m}, 2 \mathrm{H}), 7.35-7.30(\mathrm{~m}, 2 \mathrm{H}), 7.28-7.23(\mathrm{~m}, 1 \mathrm{H}), 6.63(\mathrm{~d}, J=15.8$ $\mathrm{Hz}, 1 \mathrm{H}), 6.29(\mathrm{dt}, J=15.9,6.1 \mathrm{~Hz}, 1 \mathrm{H}), 4.23(\mathrm{dd}, J=6.1,1.5 \mathrm{~Hz}, 2 \mathrm{H}), 3.82(\mathrm{t}, J=6.2 \mathrm{~Hz}, 2 \mathrm{H}), 3.51(\mathrm{t}, J=6.2 \mathrm{~Hz}$, 2H) $\mathrm{ppm}$.

${ }^{13} \mathbf{C}$ NMR (101 MHz, $\left.\mathrm{CDCl}_{3}\right): \delta=136.6,133.1,128.7,128.0,126.7,125.6,71.9,70.1,30.6 \mathrm{ppm}$.

All spectroscopic data corresponded to the reported literature values. ${ }^{[24]}$

$N$-(2-hydroxyethyl)-4-methylbenzenesulfonamide (SI-7)

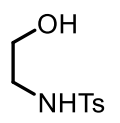

SI-7

A solution of 2-aminoethanol (2.40 mL, $2.44 \mathrm{~g}, 40.0 \mathrm{mmol}, 1.00$ equiv.) and TsCl (7.63 g, $40.0 \mathrm{mmol}, 1.00$ equiv.) in $\mathrm{CH}_{2} \mathrm{Cl}_{2}(100 \mathrm{~mL})$ was stirred for $10 \mathrm{~min}$ at $0{ }^{\circ} \mathrm{C}$, followed by the dropwise addition of $\mathrm{NEt}_{3}(5.58 \mathrm{~mL}, 4.05 \mathrm{~g}$, $40.0 \mathrm{mmol}, 1.00$ equiv.). The rection was stirred for $6 \mathrm{~h}$ at $\mathrm{rt}$ and washed with $\mathrm{H}_{2} \mathrm{O}(3 \times 100 \mathrm{~mL})$ and brine $(100$ 
$\mathrm{mL}$ ). The organic layer was dried over $\mathrm{Na}_{2} \mathrm{SO}_{4}$ and all volatiles were removed under reduced pressure to afford the title compound SI-7 (5.65 g, $26.2 \mathrm{mmol}, 66 \%$ ) as a white solid, which was used in the next step without further purification.

Analytical data of SI-7:

${ }^{1} \mathbf{H}$ NMR $\left(400 \mathrm{MHz}, \mathrm{CDCl}_{3}\right): \delta=7.76(\mathrm{~d}, J=8.3 \mathrm{~Hz}, 2 \mathrm{H}), 7.32(\mathrm{~d}, J=8.0 \mathrm{~Hz}, 3 \mathrm{H}), 5.06(\mathrm{br} \mathrm{s}, 1 \mathrm{H}), 3.69(\mathrm{~d}, J=$ $4.8 \mathrm{~Hz}, 2 \mathrm{H}), 3.09$ (d, $J=5.2 \mathrm{~Hz}, 2 \mathrm{H}), 2.43$ (s, 3H) ppm.

${ }^{13} \mathrm{C}$ NMR (101 MHz, $\left.\mathrm{CDCl}_{3}\right): \delta=143 ., 136.8,129.9$ (2C), 127.3 (2C), 61.5, 45.2, $21.7 \mathrm{ppm}$.

All spectroscopic data corresponded to the reported literature values. ${ }^{[25]}$

$N$-(2-Hydroxyethyl)-4-methyl- $N$-(3-methylbut-2-en-1-yl)benzenesulfonamide (SI-8)

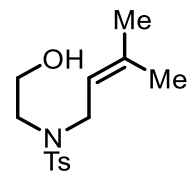

SI-8

Synthesized according to GP D from N-(2-hydroxyethyl)-4-methylbenzenesulfonamide (SI-7) (1.08 g, 5.00 mmol, 1.00 equiv.), $\mathrm{K}_{2} \mathrm{CO}_{3}$ (1.38 g, $10.0 \mathrm{mmol}, 2.00$ equiv.), $\mathrm{KI}$ ( $83 \mathrm{mg}, 500 \mu \mathrm{mol}, 5 \mathrm{~mol} \%$ ) and 1-bromo-3-methylbut2-ene $\left(847 \mu \mathrm{L}, 1.08 \mathrm{~g}, 6.50 \mathrm{mmol}, 1.30\right.$ equiv.). Purification by flash chromatography $\left(\mathrm{SiO}_{2}, n\right.$-pentane:EtOAc $=$ 3:1 - 1:1) afforded SI-8 (1.22 g, $4.31 \mathrm{mmol}, 86 \%)$ as a colourless oil.

Analytical data of SI-8:

TLC $\left(\mathrm{SiO}_{2}\right): \mathrm{R}_{f}(n$-pentane:EtOAc $=3: 1)=0.22$

${ }^{1} \mathbf{H}$ NMR (400 MHz, CDCl $): \delta=7.70(\mathrm{~d}, J=8.3 \mathrm{~Hz}, 2 \mathrm{H}), 7.31(\mathrm{~d}, J=7.9 \mathrm{~Hz}, 2 \mathrm{H}), 5.07-4.98(\mathrm{~m}, 1 \mathrm{H}), 3.83(\mathrm{~d}$, $J=7.1 \mathrm{~Hz}, 2 \mathrm{H}), 3.72(\mathrm{~m}, 2 \mathrm{H}), 3.20(\mathrm{t}, J=5.3 \mathrm{~Hz}, 2 \mathrm{H}), 2.43$ (s, 3H), $2.26(\mathrm{br} \mathrm{s}, 1 \mathrm{H}), 1.67$ (s, 3H), 1.62 (s, 3H) ppm. ${ }^{13} \mathrm{C}$ NMR (101 MHz, $\left.\mathrm{CDCl}_{3}\right): \delta=143.6,137.7,136.5,129.8$ (2C), 127.5 (2C), 119.0, 61.4, 49.9, 47.2, 25.9, 21.7, $18.0 \mathrm{ppm}$.

All spectroscopic data corresponded to the reported literature values. ${ }^{[26]}$

$N$-(2-Bromoethyl)-4-methyl- $N$-(3-methylbut-2-en-1-yl)benzenesulfonamide (2v)

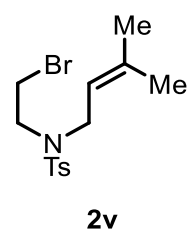

Synthesized according to GP E from $N$-(2-hydroxyethyl)-4-methylbenzenesulfonamide (SI-8) (567 mg, $2.00 \mathrm{mmol}$, 1.00 equiv.). Purification by flash chromatography $\left(\mathrm{SiO}_{2}, n\right.$-pentane:EtOAc $\left.=10: 1\right)$ afforded $\mathbf{2 v}(614 \mathrm{mg}$, $1.77 \mathrm{mmol}, 89 \%)$ as a colourless oil.

Analytical data of $2 v$ :

TLC $\left(\mathrm{SiO}_{2}\right): \mathrm{R}_{f}(n$-pentane:EtOAc $=10: 1)=0.42$

${ }^{1} \mathbf{H}$ NMR $\left(400 \mathrm{MHz}, \mathrm{CDCl}_{3}\right): \delta=7.70(\mathrm{~d}, \mathrm{~J}=8.3 \mathrm{~Hz}, 2 \mathrm{H}), 7.31(\mathrm{~d}, \mathrm{~J}=8.0 \mathrm{~Hz}, 2 \mathrm{H}), 5.06-4.98(\mathrm{~m}, 1 \mathrm{H}), 3.80(\mathrm{~d}, \mathrm{~J}$ $=7.2 \mathrm{~Hz}, 2 \mathrm{H}), 3.49-3.33(\mathrm{~m}, 4 \mathrm{H}), 2.43(\mathrm{~s}, 3 \mathrm{H}), 1.69(\mathrm{~s}, 3 \mathrm{H}), 1.63(\mathrm{~s}, 3 \mathrm{H}) \mathrm{ppm}$. 
${ }^{13}$ C NMR (101 MHz, $\left.\mathrm{CDCl}_{3}\right): \delta=143.7,138.2,136.6,129.9$ (2C), 127.4 (2C), 118.8, 49.0, 46.9, 29.8, 26.0, 21.7, $18.0 \mathrm{ppm}$.

All spectroscopic data corresponded to the reported literature values. ${ }^{[26]}$

$N$-(2-Chloroethyl)-4-methyl- $N$-(3-methylbut-2-en-1-yl)benzenesulfonamide (3v)

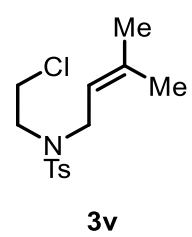

Synthesized according to GP E from $\mathrm{PPh}_{3}\left(787 \mathrm{mg}, 3.00 \mathrm{mmol}, 3.00\right.$ equiv.), $\mathrm{CCl}_{4}$ (290 $\mu \mathrm{L}, 461 \mathrm{mg}, 3.00 \mathrm{mmol}$, 3.00 equiv.) and $N$-(2-hydroxyethyl)-4-methylbenzenesulfonamide (SI-8) (283 mg, $1.00 \mathrm{mmol}, 1.00$ equiv.). Purification by flash chromatography $\left(\mathrm{SiO}_{2}, n\right.$-pentane:EtOAc $\left.=10: 1\right)$ afforded $\mathbf{3 v}(76 \mathrm{mg}, 251 \mu \mathrm{mol}, 25 \%)$ as a colourless oil.

Analytical data of $3 v$ :

TLC $\left(\mathrm{SiO}_{2}\right): \mathrm{R}_{f}(n$-pentane:EtOAc $=10: 1)=0.35$

${ }^{1} \mathbf{H}$ NMR (400 MHz, CDCl $): \delta=7.70(\mathrm{~d}, J=8.3 \mathrm{~Hz}, 2 \mathrm{H}), 7.31(\mathrm{~d}, J=7.9 \mathrm{~Hz}, 2 \mathrm{H}), 5.06-4.97(\mathrm{~m}, 1 \mathrm{H}), 3.81(\mathrm{~d}$, $J=7.2 \mathrm{~Hz}, 2 \mathrm{H}), 3.61(\mathrm{dd}, J=8.1,6.9 \mathrm{~Hz}, 2 \mathrm{H}), 3.33(\mathrm{dd}, J=8.1,6.8 \mathrm{~Hz}, 2 \mathrm{H}), 2.44(\mathrm{~s}, 3 \mathrm{H}), 1.69$ (s, $3 \mathrm{H}), 1.63$ (s, 3H) $\mathrm{ppm}$.

${ }^{13}$ C NMR (101 MHz, $\left.\mathrm{CDCl}_{3}\right): \delta=143.6,138.2,136.7,129.9$ (2C), 127.4 (2C), 118.8, 48.8, 47.0, 42.2, 26.0, 21.7, $18.0 \mathrm{ppm}$.

IR (ATR): 2971 (m), 2919 (m), 1451 (w), 1342 (m), 1158 (s), 1091 (m), 723 (m), 653 (m), 549 (m) cm-

HRMS (ESI): calcd. for $\left[\mathrm{C}_{14} \mathrm{H}_{20} \mathrm{ClNO}_{2} \mathrm{~S}+\mathrm{Na}\right]^{+}$, [M+Na] $]^{+}$: 324.0795; found: 324.0794 .

$N$-Allyl- $N$-(2-hydroxyethyl)-4-methylbenzenesulfonamide (SI-9)

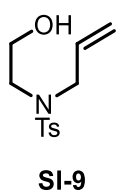

Synthesized according to GP D from $N$-(2-hydroxyethyl)-4-methylbenzenesulfonamide (SI-7) (646 g, $3.00 \mathrm{mmol}$, 1.00 equiv.), $\mathrm{K}_{2} \mathrm{CO}_{3}$ (829 mg, $6.00 \mathrm{mmol}, 2.00$ equiv.), $\mathrm{KI}$ (50 mg, $300 \mu \mathrm{mol}, 10 \mathrm{~mol} \%$ ) and 3-bromoprop-1-ene (337 $\mu \mathrm{L}, 427 \mathrm{mg}, 3.90 \mathrm{mmol}, 1.30$ equiv.). Purification by flash chromatography $\left(\mathrm{SiO}_{2}, n\right.$-pentane:EtOAc $=3: 1-$ 1:1) afforded SI-9 (734 mg, $2.88 \mathrm{mmol}, 96 \%)$ as a colourless oil.

Analytical data of SI-9:

TLC $\left(\mathrm{SiO}_{2}\right): \mathrm{R}_{f}(n$-pentane:EtOAc $=3: 1)=0.22$

${ }^{1} \mathbf{H}$ NMR (400 MHz, $\left.\mathrm{CDCl}_{3}\right): \delta=7.71(\mathrm{~d}, J=8.3 \mathrm{~Hz}, 2 \mathrm{H}), 7.32(\mathrm{~d}, J=8.0 \mathrm{~Hz}, 2 \mathrm{H}), 5.74-5.62(\mathrm{~m}, 1 \mathrm{H}), 5.22-$ $5.15(\mathrm{~m}, 2 \mathrm{H}), 3.85(\mathrm{~d}, J=6.4 \mathrm{~Hz}, 2 \mathrm{H}), 3.73(\mathrm{q}, J=5.5 \mathrm{~Hz}, 2 \mathrm{H}), 3.24(\mathrm{t}, \mathrm{J}=5.4 \mathrm{~Hz}, 2 \mathrm{H}), 2.43(\mathrm{~s}, 3 \mathrm{H}), 2.27-2.18$ (m, 1H) ppm.

${ }^{13} \mathrm{C}$ NMR (101 MHz, $\left.\mathrm{CDCl}_{3}\right): \delta=143.7,136.3,133.2,123.0$ (2C), 127.4 (2C), 119.5, 61.3, 52.4, 49.9, 21.7 ppm. All spectroscopic data corresponded to the reported literature values. ${ }^{[27]}$ 


\section{$N$-Allyl- $N$-(2-bromoethyl)-4-methylbenzenesulfonamide (2w)}

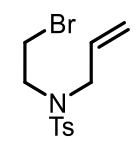

$2 \mathrm{w}$

Synthesized according to GP E from $N$-allyl- $N$-(2-hydroxyethyl)-4-methylbenzenesulfonamide (SI-9) (511 mg, $2.00 \mathrm{mmol}, 1.00$ equiv.). Purification by flash chromatography $\left(\mathrm{SiO}_{2}, n\right.$-pentane:EtOAc $\left.=10: 1\right)$ afforded $\mathbf{2 w}$ (367 $\mathrm{mg}, 1.15 \mathrm{mmol}, 58 \%)$ as a faint yellow oil.

Analytical data of $2 w$ :

$\mathbf{T L C}\left(\mathrm{SiO}_{2}\right): \mathrm{R}_{f}(n$-pentane:EtOAc $=3: 1)=0.33$

${ }^{1} \mathbf{H}$ NMR $\left(400 \mathrm{MHz}, \mathrm{CDCl}_{3}\right): \delta=7.71(\mathrm{~d}, J=8.3 \mathrm{~Hz}, 2 \mathrm{H}), 7.32(\mathrm{~d}, J=7.9 \mathrm{~Hz}, 2 \mathrm{H}), 5.72-5.62(\mathrm{~m}, 1 \mathrm{H}), 5.21$ (dq, $J=6.4,1.3 \mathrm{~Hz}, 1 \mathrm{H}), 5.18(\mathrm{t}, J=1.3 \mathrm{~Hz}, 1 \mathrm{H}), 3.81(\mathrm{~d}, J=6.5 \mathrm{~Hz}, 2 \mathrm{H}), 3.50-3.39(\mathrm{~m}, 4 \mathrm{H}), 2.44(\mathrm{~s}, 3 \mathrm{H}) \mathrm{ppm}$.

${ }^{13}$ C NMR (101 MHz, $\mathrm{CDCl}_{3}$ ): $\delta=143.9,136.4,133.0,130.0$ (2C), 127.3 (2C), 119.8, 52.2, 49.1, 29.4, 21.7 ppm. All spectroscopic data corresponded to the reported literature values. ${ }^{[27]}$

$N$-(Cyclohex-1-en-1-ylmethyl)- $N$-(2-hydroxyethyl)-4-methylbenzenesulfonamide (SI-10)

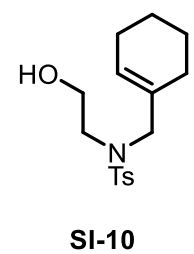

To a stirred solution of cyclohexen-1-ylmethanol (SI-2) $\left(280 \mathrm{mg}, 2.50 \mathrm{mmol}, 1.00\right.$ equiv.) in dry $\mathrm{Et}_{2} \mathrm{O}(12 \mathrm{~mL})$ was added $\mathrm{PBr}_{3}(119 \mu \mathrm{L}, 338 \mathrm{mg}, 1.25 \mathrm{mmol}, 0.50$ equiv. $)$ at $0{ }^{\circ} \mathrm{C}$. The reaction mixture was stirred for $2 \mathrm{~h}$ and added to aq. sat. $\mathrm{K}_{2} \mathrm{CO}_{3}(10 \mathrm{~mL})$. Layers were separated and the aqueous layer was extracted with $\mathrm{Et}_{2} \mathrm{O}(2 \times 10 \mathrm{~mL})$. The combined organic layers were dried over $\mathrm{MgSO}_{4}$, and all volatiles were removed under reduced pressure to afford crude 1-(bromomethyl)cyclohex-1-ene which was directly used in GP C with $N$-(2-hydroxyethyl)-4methylbenzenesulfonamide (SI-7) (503 mg, $2.50 \mathrm{mmol}, 1.00$ equiv.) and $\mathrm{K}_{2} \mathrm{CO}_{3}$ (1.04 g, 7.50 mmol, 3.00 equiv.). Purification by flash chromatography ( $\mathrm{SiO}_{2}, n$-pentane:EtOAc = 3:1) afforded SI-10 (302 mg, $\left.976 \mu \mathrm{mol}, 39 \%\right)$ as a colourless oil.

Analytical data of SI-10:

TLC $\left(\mathrm{SiO}_{2}\right): \mathrm{R}_{f}(n$-pentane: EtOAc $=3: 1)=0.63$

${ }^{1} \mathbf{H}$ NMR $\left(400 \mathrm{MHz}, \mathrm{CDCl}_{3}\right): \delta=7.70(\mathrm{~d}, J=8.3 \mathrm{~Hz}, 2 \mathrm{H}), 7.31(\mathrm{~d}, J=8.0 \mathrm{~Hz}, 2 \mathrm{H}), 5.62-5.56(\mathrm{~m}, 1 \mathrm{H}), 3.67(\mathrm{dd}$, $J=11.1,5.6 \mathrm{~Hz}, 4 \mathrm{H}), 3.16(\mathrm{t}, J=5.4 \mathrm{~Hz}, 2 \mathrm{H}), 2.43(\mathrm{~s}, 3 \mathrm{H}), 2.37-2.29(\mathrm{~m}, 1 \mathrm{H}), 2.03-1.93(\mathrm{~m}, 4 \mathrm{H}), 1.64-1.51$ $(\mathrm{m}, 4 \mathrm{H}) \mathrm{ppm}$.

${ }^{13} \mathrm{C}$ NMR (101 MHz, $\left.\mathrm{CDCl}_{3}\right): \delta=143.6,136.1,133.4,129.9$ (2C), 127.5 (2C), 127.2, 61.5, 57.0, 50.5, 26.1, 25.4, 22.5, 22.3, $21.7 \mathrm{ppm}$.

IR (ATR): 3517 (br), 2926 (m), 1446 (w), 1307 (m), 1158 (s), 655 (m), 549 (m) cm'.

HRMS (ESI): calcd. for $\left[\mathrm{C}_{16} \mathrm{H}_{23} \mathrm{NO}_{3} \mathrm{~S}+\mathrm{Na}\right]^{+},[\mathrm{M}+\mathrm{Na}]^{+}: 332.1291$; found: 332.1292 . 


\section{$N$-(2-Bromoethyl)- $N$-(cyclohex-1-en-1-ylmethyl)-4-methylbenzenesulfonamide (2x)}

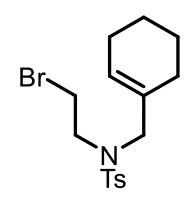

$2 x$

Synthesized according to GP E from $N$-(cyclohex-1-en-1-ylmethyl)- $N$-(2-hydroxyethyl)-4methylbenzenesulfonamide (SI-10) $\left(250 \mathrm{mg}, 808 \mu \mathrm{mol}, 1.00\right.$ equiv.). Purification by flash chromatography $\left(\mathrm{SiO}_{2}\right.$, $n$-pentane:EtOAc $=10: 1)$ afforded $\mathbf{2 x}(214 \mathrm{mg}, 574 \mu \mathrm{mol}, 71 \%)$ as a white solid.

Analytical data of $2 x$ :

TLC $\left(\mathrm{SiO}_{2}\right): \mathrm{R}_{f}(n$-pentane: EtOAc $=3: 1)=0.48$

Mp: $69.2-70.4{ }^{\circ} \mathrm{C}$.

${ }^{1} \mathbf{H}$ NMR $\left(400 \mathrm{MHz}, \mathrm{CDCl}_{3}\right): \delta=7.69(\mathrm{~d}, J=8.3 \mathrm{~Hz}, 2 \mathrm{H}), 7.31(\mathrm{~d}, J=7.7 \mathrm{~Hz}, 2 \mathrm{H}), 5.65-5.58(\mathrm{~m}, 1 \mathrm{H}), 3.62(\mathrm{~s}$, 2H), $3.43-3.29(\mathrm{~m}, 4 \mathrm{H}), 2.43(\mathrm{~s}, 3 \mathrm{H}), 2.05-1.92(\mathrm{~m}, 4 \mathrm{H}), 1.67-1.51(\mathrm{~m}, 4 \mathrm{H}) \mathrm{ppm}$.

${ }^{13}$ C NMR (101 MHz, $\left.\mathrm{CDCl}_{3}\right): \delta=143.7,136.4,133.2,129.9$ (2C), 127.7, 127.3 (2C), 56.5, 49.5, 29.4, 26.1, 25.4, 22.6, 22.3, $21.7 \mathrm{ppm}$.

IR (ATR): 2927 (m), 1447 (w), 1340 (s), 1160 (s), 1091 (w), 932 (w), 549 (m) cm-1.

HRMS (ESI): calcd. for $\left[\mathrm{C}_{16} \mathrm{H}_{22} \mathrm{BrNOS}+\mathrm{H}\right]^{+},[\mathrm{M}+\mathrm{H}]^{+}: 372.0627$; found: 372.0627 .

( \pm )- $N$-(2-Hydroxypropyl)-4-methylbenzenesulfonamide (SI-11)

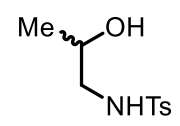

SI-11

A solution of 1-aminopropan-2-ol (3.10 mL, $3.00 \mathrm{~g}, 40.0 \mathrm{mmol}, 1.00$ equiv.) and TsCl (7.63 g, $40.0 \mathrm{mmol}, 1.00$ equiv.) in $\mathrm{CH}_{2} \mathrm{Cl}_{2}(100 \mathrm{~mL})$ was stirred for $10 \mathrm{~min}$ at $0{ }^{\circ} \mathrm{C}$, followed by the dropwise addition of $\mathrm{NEt}_{3}(5.58 \mathrm{~mL}$, $4.05 \mathrm{~g}, 40.0 \mathrm{mmol}, 1.00$ equiv.). The reaction was stirred for $18 \mathrm{~h}$ at $\mathrm{rt}$ and washed with $\mathrm{H}_{2} \mathrm{O}(3 \times 100 \mathrm{~mL})$ and brine $(100 \mathrm{~mL})$. The organic layer was dried over $\mathrm{Na}_{2} \mathrm{SO}_{4}$ and all volatiles were removed under reduced pressure to afford the title compound SI-11 (7.30 g, $31.8 \mathrm{mmol}, 80 \%)$ as a white solid, which was used in the next step without further purification.

Analytical data of SI-11:

${ }^{1} \mathbf{H}$ NMR $\left(400 \mathrm{MHz}, \mathrm{CDCl}_{3}\right): \delta=7.75(\mathrm{~d}, \mathrm{~J}=8.3 \mathrm{~Hz}, 2 \mathrm{H}), 7.32(\mathrm{~d}, \mathrm{~J}=8.2 \mathrm{~Hz}, 2 \mathrm{H}), 4.88(\mathrm{~s}, 1 \mathrm{H}), 3.97-3.84(\mathrm{~m}$, 1H), $3.11-3.00(\mathrm{~m}, 1 \mathrm{H}), 2.77$ (dd, J = 13.1, $8.1 \mathrm{~Hz}, 1 \mathrm{H}), 2.43(\mathrm{~s}, 3 \mathrm{H}), 1.91(\mathrm{~s}, 1 \mathrm{H}), 1.16(\mathrm{~d}, \mathrm{~J}=6.3 \mathrm{~Hz}, 3 \mathrm{H}) \mathrm{ppm}$. ${ }^{13}$ C NMR (101 MHz, $\left.\mathrm{CDCl}_{3}\right): \delta=143.7,136.8,129.9$ (2C), 127.2 (2C), 66.7, 50.1, 21.7, 20.9 ppm.

All spectroscopic data corresponded to the reported literature values. ${ }^{[25]}$ 


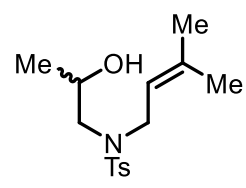

SI-12

Synthesized according to GP D from $N$-(2-hydroxypropyl)-4-methylbenzenesulfonamide (SI-11) (688 g, 3.00 mmol, 1.00 equiv.), $\mathrm{K}_{2} \mathrm{CO}_{3}$ (829 mg, $6.00 \mathrm{mmol}, 2.00$ equiv.), $\mathrm{KI}$ (50 mg, $300 \mu \mathrm{mol}, 10 \mathrm{~mol} \%$ ) and 3-bromoprop1-ene $\left(337 \mu \mathrm{L}, 427 \mathrm{mg}, 3.90 \mathrm{mmol}, 1.30\right.$ equiv.). Purification by flash chromatography $\left(\mathrm{SiO}_{2}, n\right.$-pentane:EtOAc $=$ 3:1) afforded SI-12 (651 $\mathrm{mg}, 2.19 \mathrm{mmol}, 73 \%)$ as a colourless oil.

Analytical data of SI-12:

TLC $\left(\mathrm{SiO}_{2}\right): \mathrm{R}_{f}(n$-pentane:EtOAc $=3: 1)=0.42$

${ }^{1} \mathbf{H}$ NMR $\left(400 \mathrm{MHz}, \mathrm{CDCl}_{3}\right): \delta=7.70(\mathrm{~d}, J=8.3 \mathrm{~Hz}, 1 \mathrm{H}), 7.31(\mathrm{~d}, J=8.0 \mathrm{~Hz}, 1 \mathrm{H}), 5.05-4.95(\mathrm{~m}, 1 \mathrm{H}), 4.05-$ $3.92(\mathrm{~m}, 1 \mathrm{H}), 3.84(\mathrm{~d}, J=7.1 \mathrm{~Hz}, 2 \mathrm{H}), 3.05(\mathrm{dd}, J=14.6,8.6 \mathrm{~Hz}, 1 \mathrm{H}), 2.93(\mathrm{dd}, J=14.6,3.1 \mathrm{~Hz}, 1 \mathrm{H}), 2.60$ (br s, $1 \mathrm{H}), 2.43(\mathrm{~s}, 3 \mathrm{H}), 1.66(\mathrm{~s}, 2 \mathrm{H}), 1.61(\mathrm{~s}, 3 \mathrm{H}), 1.14(\mathrm{~d}, J=6.3 \mathrm{~Hz}, 3 \mathrm{H}) \mathrm{ppm}$.

${ }^{13} \mathrm{C}$ NMR (101 MHz, $\left.\mathrm{CDCl}_{3}\right): \delta=143.6,137.7,136.5,129.8$ (2C), 127.5 (2C), 118.9, 66.4, 55.3, 47.6, 25.9, 21.7, 20.5, $17.9 \mathrm{ppm}$.

All spectroscopic data corresponded to the reported literature values. ${ }^{[12]}$

( \pm )- $N$-(2-Bromopropyl)-4-methyl- $N$-(3-methylbut-2-en-1-yl)benzenesulfonamide (2y)

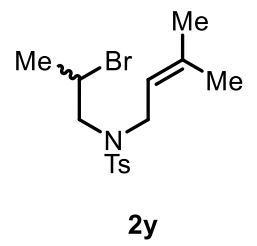

Synthesized according to GP E from $N$-(2-hydroxypropyl)-4-methyl- $N$-(3-methylbut-2-en-1yl)benzenesulfonamide (SI-12) $\left(297 \mathrm{mg}, 1.00 \mathrm{mmol}, 1.00\right.$ equiv.). Purification by flash chromatography $\left(\mathrm{SiO}_{2}\right.$, $n$-pentane:EtOAc $=10: 1)$ afforded $\mathbf{2 y}(270 \mathrm{mg}, 749 \mu \mathrm{mol}, 75 \%)$ as a colourless gum.

Analytical data of $\mathbf{2 y}$ :

TLC $\left(\mathrm{SiO}_{2}\right): \mathrm{R}_{f}(n$-pentane:EtOAc $=3: 1)=0.55$

${ }^{1} \mathbf{H}$ NMR (400 MHz, CDCl $): \delta=7.69(\mathrm{~d}, J=8.3 \mathrm{~Hz}, 2 \mathrm{H}), 7.31(\mathrm{~d}, J=7.7 \mathrm{~Hz}, 2 \mathrm{H}), 4.98-4.88(\mathrm{~m}, 1 \mathrm{H}), 4.35-$ $4.20(\mathrm{~m}, 1 \mathrm{H}), 3.93-3.72(\mathrm{~m}, 2 \mathrm{H}), 3.38(\mathrm{dd}, J=14.5,8.4 \mathrm{~Hz}, 1 \mathrm{H}), 3.29(\mathrm{dd}, J=14.6,6.1 \mathrm{~Hz}, 1 \mathrm{H}), 2.43(\mathrm{~s}, 3 \mathrm{H})$, $1.72(\mathrm{~d}, J=6.7 \mathrm{~Hz}, 3 \mathrm{H}), 1.65(\mathrm{~s}, 3 \mathrm{H}), 1.62(\mathrm{~s}, 3 \mathrm{H}) \mathrm{ppm}$.

${ }^{13} \mathrm{C}$ NMR (101 MHz, $\left.\mathrm{CDCl}_{3}\right): \delta=143.6,137.9,136.5,129.8$ (2C), 127.5 (2C), 118.6, 55.3, 47.4, 47.3, 25.9, 23.5, 21.7, $17.9 \mathrm{ppm}$.

All spectroscopic data corresponded to the reported literature values. ${ }^{[12]}$ 


\section{4-Methyl- $N$-(2,2,2-trichloroethyl)benzenesulfonamide (SI-13)}

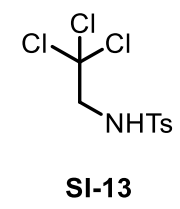

A solution of 2,2,2-trichloroethan-1-amine ${ }^{[28]}(6.12 \mathrm{~mL}, 5.94 \mathrm{~g}, 40.0 \mathrm{mmol}, 1.00$ equiv.) and TsCl (7.63 g, 40.0 mmol, 1.00 equiv.) in $\mathrm{CH}_{2} \mathrm{Cl}_{2}(100 \mathrm{~mL})$ was stirred for $10 \mathrm{~min}$ at $0{ }^{\circ} \mathrm{C}$, followed by the dropwise addition of $\mathrm{NEt}_{3}$ (5.58 mL, $4.05 \mathrm{~g}, 40.0 \mathrm{mmol}, 1.00$ equiv.). The rection was stirred for $17 \mathrm{~h}$ at $\mathrm{rt}$ and washed with $\mathrm{H}_{2} \mathrm{O}(3 \times 100$ $\mathrm{mL})$ and brine $(100 \mathrm{~mL})$. The organic layer was dried over $\mathrm{Na}_{2} \mathrm{SO}_{4}$ and all volatiles were removed under reduced pressure to afford a brown solid, which was purified in a first step by flash chromatography $\left(\mathrm{SiO}_{2}, n\right.$-pentane:EtOAc $=10: 1-3: 1)$ to afford an off white solid. Recrystallization from EtOAc at $-33^{\circ} \mathrm{C}$ afforded SI-13 $(5.86 \mathrm{~g}, 19.4 \mathrm{mmol}$, $48 \%)$ as a white solid.

Analytical data of $\mathbf{S I - 1 3 :}$

TLC $\left(\mathrm{SiO}_{2}\right): \mathrm{R}_{f}(n$-pentane: EtOAc $=10: 1)=0.17$

Mp: $140.1-141.8^{\circ} \mathrm{C}$.

${ }^{1} \mathbf{H}$ NMR $\left(400 \mathrm{MHz}, \mathrm{CDCl}_{3}\right): \delta=7.79(\mathrm{~d}, J=8.3 \mathrm{~Hz}, 2 \mathrm{H}), 7.33(\mathrm{~d}, J=7.9 \mathrm{~Hz}, 2 \mathrm{H}), 5.30(\mathrm{t}, J=7.0 \mathrm{~Hz}, 1 \mathrm{H}), 3.97$ (d, $J=7.0 \mathrm{~Hz}, 2 \mathrm{H}), 2.44$ (s, 3H) ppm.

${ }^{13}$ C NMR (101 MHz, $\left.\mathrm{CDCl}_{3}\right): \delta=144.3,137.4,130.0$ (2C), 127.2 (2C), 96.9, 61.2, $21.7 \mathrm{ppm}$.

IR (ATR): 3255 (br), 2972 (m), 2901 (m), 1447 (w), 1324 (m), 1158 (s), 1046 (s), 877 (m), 707 (m), 534 (m) cm . HRMS (ESI): calcd. for $\left[\mathrm{C}_{9} \mathrm{H}_{10} \mathrm{Cl}_{3} \mathrm{NO}_{2} \mathrm{~S}+\mathrm{H}\right]^{+},[\mathrm{M}+\mathrm{H}]^{+}: 301.9571$; found: 301.9569 .

\section{4-Methyl- $N$-(3-methylbut-2-en-1-yl)- $N$-(2,2,2-trichloroethyl)benzenesulfonamide (3z)}

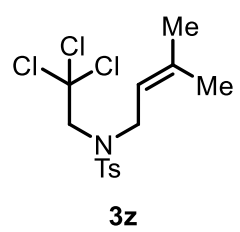

Synthesized according to GP C from 4-methyl- $N$-(2,2,2-trichloroethyl)benzenesulfonamide (SI-13) (908 mg, $3.00 \mathrm{mmol}, 1.00$ equiv.), $\mathrm{K}_{2} \mathrm{CO}_{3}$ (1.24 g, $9.00 \mathrm{mmol}, 3.00$ equiv.) and 3-bromo-2-methylprop-1-ene (332 $\mu \mathrm{L}, 446$ $\mathrm{mg}, 3.30 \mathrm{mmol}, 1.10$ equiv. $)$. Purification by flash chromatography $\left(\mathrm{SiO}_{2}, n\right.$-pentane:EtOAc $\left.=10: 1\right)$ afforded $\mathbf{3 z}$ (1.03 g, $2.78 \mathrm{mmol}, 93 \%)$ as a white solid.

Analytical data of $\mathbf{3 z}$ :

TLC $\left(\mathrm{SiO}_{2}\right): \mathrm{R}_{f}(n$-pentane: EtOAc $=10: 1)=0.59$

Mp: $68.8-69.8^{\circ} \mathrm{C}$.

${ }^{1}$ H NMR (400 MHz, $\left.\mathrm{CDCl}_{3}\right): \delta=7.79-7.75(\mathrm{~m}, 2 \mathrm{H}), 7.32-7.30(\mathrm{~m}, 2 \mathrm{H}), 4.87-4.79(\mathrm{~m}, 1 \mathrm{H}), 4.28(\mathrm{~s}, 2 \mathrm{H}), 4.15$ (d, $J=7.0 \mathrm{~Hz}, 2 \mathrm{H}), 2.44$ (s, 3H), 1.65 (s, 3H), 1.59 (s, 3H) ppm.

${ }^{13}$ C NMR (101 MHz, $\left.\mathrm{CDCl}_{3}\right): \delta=144.0,138.9,137.4,129.8$ (2C), 127.9 (2C), 118.1, 98.7, 62.6, 47.3, 26.1, 21.7, $18.1 \mathrm{ppm}$.

IR (ATR): 2926 (w), 1447 (w), 1340 (m), 1160 (s), 1092 (m), 907 (s), 814 (s), 732 (s), 546 (m) cm¹.

HRMS (ESI): calcd. for $\left[\mathrm{C}_{14} \mathrm{H}_{18} \mathrm{Cl}_{3} \mathrm{NO}_{2} \mathrm{~S}+\mathrm{Na}\right]^{+},[\mathrm{M}+\mathrm{Na}]^{+}$: 392.0016 ; found: 392.0016. 


\section{4-Methyl- $N$-(4-methylpent-3-en-1-yl)- $N$-(2,2,2-trichloroethyl)benzenesulfonamide (3za)}

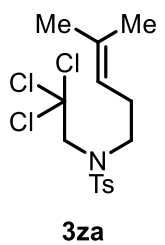

Synthesized according to GP C from 4-methyl- $N$-(2,2,2-trichloroethyl)benzenesulfonamide (SI-13) (605 mg, $2.00 \mathrm{mmol}, 1.00$ equiv.), $\mathrm{K}_{2} \mathrm{CO}_{3}$ (829 mg, $6.00 \mathrm{mmol}, 3.00$ equiv.) and 5-bromo-2-methylpent-2-ene (295 $\mu \mathrm{L}, 359$ mg, 2.20 mmol, 1.10 equiv.). Purification by flash chromatography $\left(\mathrm{SiO}_{2}, n\right.$-pentane:EtOAc $\left.=10: 1\right)$ afforded 3za (474 $\mathrm{mg}, 1.23 \mathrm{mmol}, 62 \%$ ) as a white solid.

Analytical data of $\mathbf{3 z a}$ :

TLC $\left(\mathrm{SiO}_{2}\right): \mathrm{R}_{f}(n$-pentane: EtOAc $=10: 1)=0.52$

Mp: $67.7-69.9^{\circ} \mathrm{C}$.

${ }^{1} \mathbf{H}$ NMR $\left(400 \mathrm{MHz}, \mathrm{CDCl}_{3}\right): \delta=7.78-7.70(\mathrm{~m}, 2 \mathrm{H}), 7.36-7.29(\mathrm{~m}, 2 \mathrm{H}), 4.89-4.80(\mathrm{~m}, 1 \mathrm{H}), 4.30(\mathrm{~s}, 2 \mathrm{H}), 3.41$ $-3.32(\mathrm{~m}, 2 \mathrm{H}), 2.44(\mathrm{~s}, 3 \mathrm{H}), 2.37-2.26(\mathrm{~m}, 2 \mathrm{H}), 1.63(\mathrm{~s}, 3 \mathrm{H}), 1.55(\mathrm{~s}, 3 \mathrm{H}) \mathrm{ppm}$.

${ }^{13}$ C NMR (101 MHz, $\mathrm{CDCl}_{3}$ ): $\delta=144.0,136.8,135.0,129.9$ (2C), 127.7 (2C), 119.5, 98.4, 64.4, 49.0, 26.9, 25.8, 21.7, $17.9 \mathrm{ppm}$.

IR (ATR): 2971 (w), 2922 (w), 1598 (w), 1342 (s), 1162 (s), 1092 (m), 923 (m), 815 (m), 727 (s), 653 (m), $548(\mathrm{~s}) \mathrm{cm}^{-1}$.

HRMS (ESI): calcd. for $\left[\mathrm{C}_{15} \mathrm{H}_{20} \mathrm{Cl}_{3} \mathrm{NO}_{2} \mathrm{~S}+\mathrm{H}\right]^{+},[\mathrm{M}+\mathrm{H}]^{+}$: 384.0353; found: 384. 0362.

\section{1-Bromo-2-((3-methylbut-2-en-1-yl)oxy)-4-nitrobenzene (SI-14)}

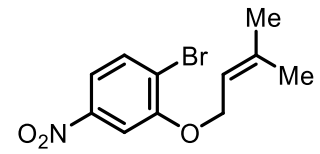

SI-14

Synthesized according to GP C from 2-bromo-5-nitrophenol (323 $\mu \mathrm{L}, 527 \mathrm{mg}, 2.42 \mathrm{mmol}, 1.00$ equiv.), $\mathrm{K}_{2} \mathrm{CO}_{3}$ (1.00 g, $7.25 \mathrm{mmol}, 3.00$ equiv.) and 1-bromo-3-methylbut-2-ene (312 $\mu \mathrm{L}, 396 \mathrm{mg}, 2.66 \mathrm{mmol}, 1.10$ equiv.). Purification by flash chromatography $\left(\mathrm{SiO}_{2}, n\right.$-pentane:EtOAc = 20:1) afforded SI-14 (599 mg, 2.09 mmol, 87\%) as an off white solid.

\section{Analytical data of SI-14:}

TLC $\left(\mathrm{SiO}_{2}\right): \mathrm{R}_{f}(n$-pentane:EtOAc $=20: 1)=0.57$

Mp: $65.8-67.7^{\circ} \mathrm{C}$.

${ }^{1} \mathbf{H}$ NMR $\left(400 \mathrm{MHz}, \mathrm{CDCl}_{3}\right): \delta=7.75-7.67(\mathrm{~m}, 3 \mathrm{H}), 5.55-5.40(\mathrm{~m}, 1 \mathrm{H}), 4.70(\mathrm{~d}, J=6.7 \mathrm{~Hz}, 2 \mathrm{H}), 1.84-1.78$ $(\mathrm{m}, 6 \mathrm{H}) \mathrm{ppm}$.

${ }^{13} \mathrm{C}$ NMR (101 MHz, $\left.\mathrm{CDCl}_{3}\right): \delta=155.8,148.1,140.0,133.7,120.3,118.2,116.5,108.0,66.9,26.0,18.6 \mathrm{ppm}$. IR (ATR): 2970 (w), 2914 (w), 1569 (w), 1512 (s), 1473 (w), 1352 (m) 1262 (m), 1038 (m), 987 (s), 864 (m), 736 (s) $\mathrm{cm}^{-1}$.

HRMS (APPI/LTQ-Orbitrap): calcd. for $\left[\mathrm{C}_{11} \mathrm{H}_{12} \mathrm{BrNO}_{3}\right]^{+},[\mathrm{M}]^{+}:$284.9995; found: 284.9988 . 


\section{2-((3-Methylbut-2-en-1-yl)oxy)benzaldehyde (SI-15)}

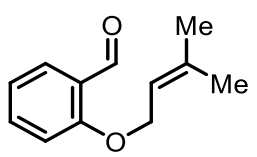

SI-15

To a stirred solution of 3-methylbut-2-en-1-ol $(2.23 \mathrm{~mL}, 1.89 \mathrm{~g}, 22.0 \mathrm{mmol}, 1.10$ equiv. $)$ in $\operatorname{dry}^{\mathrm{Et}} \mathrm{t}_{2} \mathrm{O}(11 \mathrm{~mL})$ was added $\mathrm{PBr}_{3}\left(2.09 \mathrm{~mL}, 5.96 \mathrm{~g}, 22.0 \mathrm{mmol}, 1.10\right.$ equiv.) at $0{ }^{\circ} \mathrm{C}$. The mixture was stirred for $1 \mathrm{~h}$ until TLC control showed complete consumption of the alcohol. The reaction mixture was carefully quenched by the addition of sat. aq. $\mathrm{NH}_{4} \mathrm{Cl}(15 \mathrm{~mL})$ and the layers were separated. The organic layer, containing the corresponding bromide, was directly used in GP C with 2-hydroxybenzaldehyde (2.09 mL, $2.44 \mathrm{~g}, 20.0 \mathrm{mmol}, 1.00$ equiv.) and $\mathrm{K}_{2} \mathrm{CO}_{3}(11.1 \mathrm{~g}$, $80.0 \mathrm{mmol}, 4.00$ equiv.). Purification by flash chromatography $\left(\mathrm{SiO}_{2}, n\right.$-pentane:EtOAc $\left.=10: 1\right)$ afforded SI-15 (3.03 g, $15.9 \mathrm{mmol}, 80 \%)$ as a yellow liquid.

Analytical data of SI-15:

TLC $\left(\mathrm{SiO}_{2}\right): \mathrm{R}_{f}(n$-pentane:EtOAc $=10: 1)=0.56$

${ }^{1} \mathbf{H}$ NMR $\left(400 \mathrm{MHz}, \mathrm{CDCl}_{3}\right): \delta=10.50(\mathrm{~s}, 1 \mathrm{H}), 7.83(\mathrm{dd}, J=7.7,1.9 \mathrm{~Hz}, 1 \mathrm{H}), 7.52(\mathrm{ddd}, J=8.4,7.3,1.9 \mathrm{~Hz}, 1 \mathrm{H})$, $7.05-6.95(\mathrm{~m}, 2 \mathrm{H}), 5.54-5.46(\mathrm{~m}, 1 \mathrm{H}), 4.64(\mathrm{~d}, J=6.3 \mathrm{~Hz}, 2 \mathrm{H}), 1.81(\mathrm{~s}, 3 \mathrm{H}), 1.76(\mathrm{~s}, 3 \mathrm{H}) \mathrm{ppm}$.

${ }^{13}$ C NMR (101 MHz, $\left.\mathrm{CDCl}_{3}\right): \delta=190.2,161.5,138.9,135.9,128.4,125.3,120.7,119.1,113.1,65.6,25.9$, $18.4 \mathrm{ppm}$.

All spectroscopic data corresponded to the reported literature values. ${ }^{[29]}$

(2-((3-Methylbut-2-en-1-yl)oxy)phenyl)methanol (SI-16)

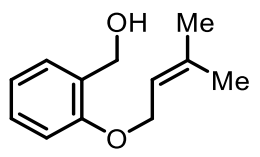

SI-16

To a stirred solution of 2-(3-methylbut-2-enoxy)benzaldehyde (SI-15) (1.04 g, $5.48 \mathrm{mmol}, 1.00$ equiv.) in dry $\mathrm{MeOH}(20 \mathrm{~mL})$ was added $\mathrm{NaBH}_{4}\left(207 \mathrm{mg}, 5.48 \mathrm{mmol}, 1.00\right.$ equiv.) at $0{ }^{\circ} \mathrm{C}$. The reaction mixture was allowed to reach $\mathrm{rt}$ and stirred for $18 \mathrm{~h}$, followed by the addition of EtOAc $(20 \mathrm{~mL})$ and sat. aq. $\mathrm{NH}_{4} \mathrm{Cl}(20 \mathrm{~mL})$. The resulting mixture was extracted with EtOAc $(2 \times 20 \mathrm{~mL})$. Combined organic layers were dried over $\mathrm{Na}_{2} \mathrm{SO}_{4}$, volatiles were removed under reduced pressure and the residue was purified by flash chromatography $\left(\mathrm{SiO}_{2}, n\right.$-pentane:EtOAc $=$ 3:1) to afford SI-16 (942 $\mathrm{mg}, 4.90 \mathrm{mmol}, 89 \%)$ as a colourless liquid.

Analytical data of SI-16:

TLC $\left(\mathrm{SiO}_{2}\right): \mathrm{R}_{f}(n$-pentane:EtOAc $=3: 1)=0.78$

${ }^{1} \mathbf{H}$ NMR (400 MHz, $\left.\mathrm{CDCl}_{3}\right): \delta=7.30-7.21(\mathrm{~m}, 2 \mathrm{H}), 6.98-6.86(\mathrm{~m}, 2 \mathrm{H}), 5.53-5.44(\mathrm{~m}, 1 \mathrm{H}), 4.69(\mathrm{~d}, J=6.4$ $\mathrm{Hz}, 2 \mathrm{H}), 4.57(\mathrm{~d}, J=6.6 \mathrm{~Hz}, 2 \mathrm{H}), 2.49(\mathrm{t}, J=6.6 \mathrm{~Hz}, 1 \mathrm{H}), 1.80$ (s, 3H), $1.74(\mathrm{~s}, 3 \mathrm{H}) \mathrm{ppm}$.

${ }^{13} \mathrm{C}$ NMR (101 MHz, $\left.\mathrm{CDCl}_{3}\right): \delta=157.0,138.4,129.5,129.0,128.9,120.7,119.7,111.6,65.1,62.7,25.9,18.4$ ppm.

IR (ATR): 3385 (br), 2972 (w), 2914 (m), 1602 (m), 1490 (m), 1454 (s), 1382 (w), 1232 (s), 1043 (m), 1003 (s), $751(\mathrm{~s}) \mathrm{cm}^{-1}$. 
HRMS (ESI): calcd. for $\left[\mathrm{C}_{12} \mathrm{H}_{16} \mathrm{O}_{2}+\mathrm{Na}\right]^{+},[\mathrm{M}+\mathrm{Na}]^{+}:$215.1043; found: 215.1041 .

1-(Bromomethyl)-2-((3-methylbut-2-en-1-yl)oxy)benzene (SI-17)

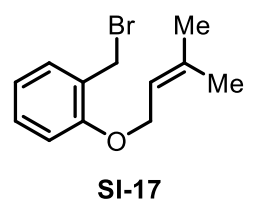

Synthesized according to GP E from (2-((3-methylbut-2-en-1-yl)oxy)phenyl)methanol (SI-16) (385 mg, $2.00 \mathrm{mmol}, 1.00$ equiv.). Purification by flash chromatography $\left(\mathrm{SiO}_{2}, n\right.$-pentane: $\left.\mathrm{CH}_{2} \mathrm{Cl}_{2}=8: 1\right)$ afforded $\mathbf{S I - 1 7}$ (145 mg, $568 \mu \mathrm{mol}, 28 \%)$ as a colourless oil.

Analytical data of SI-17:

TLC $\left(\mathrm{SiO}_{2}\right): \mathrm{R}_{f}\left(n\right.$-pentane: $\left.\mathrm{CH}_{2} \mathrm{Cl}_{2}=8: 1\right)=0.30$

${ }^{1} \mathbf{H}$ NMR (400 MHz, CDCl $): \delta=7.34(\mathrm{dd}, J=7.5,1.8 \mathrm{~Hz}, 1 \mathrm{H}), 7.31-7.22(\mathrm{~m}, 1 \mathrm{H}), 6.96-6.85(\mathrm{~m}, 2 \mathrm{H}), 5.56-$ $5.47(\mathrm{~m}, 1 \mathrm{H}), 4.63-4.57(\mathrm{~m}, 2 \mathrm{H}), 4.59(\mathrm{~s}, 2 \mathrm{H}), 1.80(\mathrm{~s}, 3 \mathrm{H}), 1.76(\mathrm{~s}, 3 \mathrm{H}) \mathrm{ppm}$.

${ }^{13}$ C NMR (101 MHz, $\left.\mathrm{CDCl}_{3}\right): \delta=156.9,137.9,131.1,130.2,126.6,120.7,119.9,112.5,65.5,29.4,25.9,18.5$ ppm.

IR (ATR): 2973 (s), 2912 (m), 1601 (m), 1491 (s), 1455 (s), 1230 (s), 1047 (m), 1001 (m), 751 (s), 607 (w) cm². HRMS (APPI/LTQ-Orbitrap): calcd. for $\left[\mathrm{C}_{12} \mathrm{H}_{15} \mathrm{BrO}\right]^{+},[\mathrm{M}]^{+}: 254.0301$; found: 254.0298. 


\subsection{Intramolecular Reductive Cyclization}

\section{General Procedure F:}

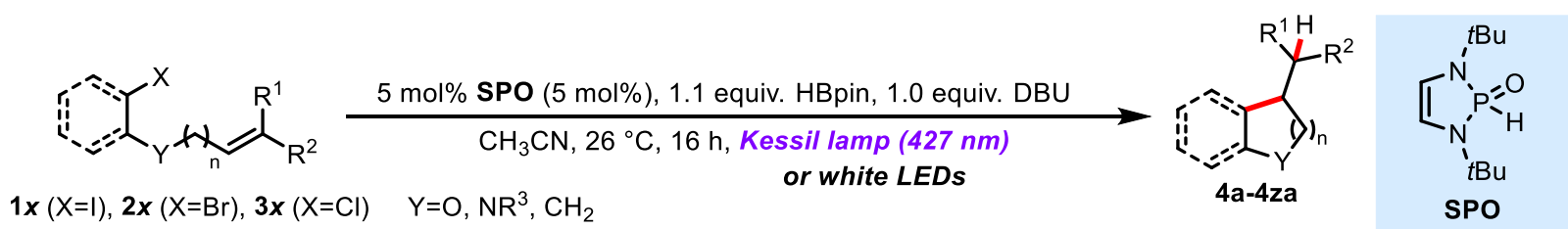

An oven-dried microwave vial, equipped with a stir bar was charged with the aryl or alkyl halide (200 $\mu$ mol, 1.00 equiv.) and transferred into the glovebox. $\mathrm{MeCN}$ ( $0.2 \mathrm{M}$ for iodides; $0.8 \mathrm{M}$ for bromides and chlorides) was added, followed by DBU (30.0 $\mu \mathrm{L}, 30.5 \mathrm{mg}, 200 \mu \mathrm{mol}, 1.00$ equiv.), SPO (43.3 $\mu \mathrm{L}$, as a $50 \mathrm{mg} / \mathrm{mL} \mathrm{MeCN}$ stock solution, $2.16 \mathrm{mg}, 10.0 \mu \mathrm{mol}, 5 \mathrm{~mol} \%)$ and HBpin $(32.0 \mu \mathrm{L}, 28.2 \mathrm{mg}, 220 \mu \mathrm{mol}, 1.10$ equiv.), unless otherwise stated. The vial was closed, removed from the glovebox, and irradiated (for iodides: white LEDs; for bromides and chlorides: Kessil lamp $427 \mathrm{~nm}$ ) for $16 \mathrm{~h}$ at $26^{\circ} \mathrm{C}$. Volatiles were removed under reduced pressure and the residue was purified by flash chromatography $\left(\mathrm{SiO}_{2}, n\right.$-pentane:EtOAc).

\section{3-Isopropyl-2,3-dihydrobenzofuran (4a)}

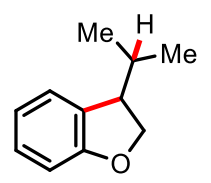

$4 a$

Synthesized according to GP F (white LEDs) from 1-iodo-2-((3-methylbut-2-en-1-yl)oxy)benzene (1a) (57.6 mg, $200 \mu \mathrm{mol}, 1.00$ equiv. $)$. Purification by flash chromatography $\left(\mathrm{SiO}_{2}, n\right.$-pentane:EtOAc $\left.=40: 1\right)$ afforded $4 \mathbf{a}(30 \mathrm{mg}$, $184 \mu \mathrm{mol}, 92 \%)$ as a colourless liquid.

Synthesized according to GP F (Kessil lamp $427 \mathrm{~nm}$ ) from 1-bromo-2-((3-methylbut-2-en-1-yl)oxy)benzene (2a) (48.2 mg, $200 \mu$ mol, 1.00 equiv.). Purification by flash chromatography $\left(\mathrm{SiO}_{2}, n\right.$-pentane:EtOAc $\left.=40: 1\right)$ afforded 4a (31 mg, $191 \mu \mathrm{mol}, 96 \%)$ as a colourless liquid.

Analytical data of $\mathbf{4 a}$ :

TLC $\left(\mathrm{SiO}_{2}\right): \mathrm{R}_{f}(n$-pentane:EtOAc $=40: 1)=0.43$

${ }^{1}$ H NMR $\left(400 \mathrm{MHz}, \mathrm{CDCl}_{3}\right): \delta=7.22-7.15(\mathrm{~m}, 1 \mathrm{H}), 7.17-7.08(\mathrm{~m}, 1 \mathrm{H}), 6.85(\mathrm{td}, J=7.4,1.0 \mathrm{~Hz}, 1 \mathrm{H}), 6.81-$ $6.74(\mathrm{~m}, 1 \mathrm{H}), 4.52(\mathrm{t}, J=9.1 \mathrm{~Hz}, 1 \mathrm{H}), 4.38(\mathrm{dd}, J=9.0,5.1 \mathrm{~Hz}, 1 \mathrm{H}), 3.38-3.28(\mathrm{~m}, 1 \mathrm{H}), 2.05-1.89(\mathrm{~m}, 1 \mathrm{H})$, $0.96(\mathrm{~d}, J=6.8 \mathrm{~Hz}, 3 \mathrm{H}), 0.88(\mathrm{~d}, J=6.8 \mathrm{~Hz}, 3 \mathrm{H}) \mathrm{ppm}$.

${ }^{13} \mathrm{C}$ NMR (101 MHz, $\left.\mathrm{CDCl}_{3}\right): \delta=160.5,129.6,128.3,125.2,120.2,109.5,74.0,48.3,31.8,20.0,18.6$ ppm. All spectroscopic data corresponded to the reported literature values. ${ }^{[30]}$ 


\section{3-Isopropyl-6-methoxy-2,3-dihydrobenzofuran (4b)}

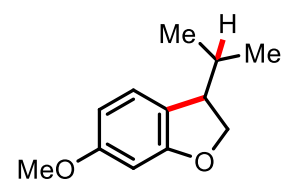

4b

Synthesized according to GP F (Kessil lamp $427 \mathrm{~nm}$ ) from 1-bromo-4-methoxy-2-((3-methylbut-2-en-1yl)oxy)benzene (2b) $\left(54.2 \mathrm{mg}, 200 \mu \mathrm{mol}, 1.00\right.$ equiv.). Purification by flash chromatography $\left(\mathrm{SiO}_{2}\right.$, $n$-pentane:EtOAc $=40: 1)$ afforded $\mathbf{4 b}(34 \mathrm{mg}, 177 \mu \mathrm{mol}, 88 \%)$ as a colourless liquid.

Analytical data of $\mathbf{4 b}$ :

TLC $\left(\mathrm{SiO}_{2}\right): \mathrm{R}_{f}(n$-pentane:EtOAc $=40: 1)=0.29$

${ }^{1} \mathbf{H}$ NMR $\left(400 \mathrm{MHz}, \mathrm{CDCl}_{3}\right): \delta=6.79-6.74(\mathrm{~m}, 1 \mathrm{H}), 6.69-6.64(\mathrm{~m}, 2 \mathrm{H}), 4.50(\mathrm{t}, J=9.0 \mathrm{~Hz}, 1 \mathrm{H}), 4.36(\mathrm{dd}, J=$ 9.0, $5.2 \mathrm{~Hz}, 1 \mathrm{H}), 3.76(\mathrm{~s}, 3 \mathrm{H}), 3.35-3.25(\mathrm{~m}, 1 \mathrm{H}), 2.03-1.90(\mathrm{~m}, 1 \mathrm{H}), 0.96(\mathrm{~d}, J=6.8 \mathrm{~Hz}, 3 \mathrm{H}), 0.88(\mathrm{~d}, J=6.9$ $\mathrm{Hz}, 3 \mathrm{H}) \mathrm{ppm}$.

${ }^{13}$ C NMR (101 MHz, $\left.\mathrm{CDCl}_{3}\right): \delta=154.7,154.0,130.7,112.9,111.7,109.2,74.2,56.2,48.7,31.7,20.0,18.5 \mathrm{ppm}$.

IR (ATR): 2957 (m), 2901 (w), 1603 (w), 1487 (s), 1464 (m), 1274 (m), 1216 (m), 1194 (m), 1179 (m), 1034 (m), $959(\mathrm{~m}), 804(\mathrm{~m}), 713(\mathrm{w}) \mathrm{cm}^{-1}$.

HRMS (ESI): calcd. for $\left[\mathrm{C}_{12} \mathrm{H}_{16} \mathrm{O}_{2}+\mathrm{H}\right]^{+},[\mathrm{M}+\mathrm{H}]^{+}:$193.1223; found: 193.1199 .

\section{3-Isopropyl-5-(trifluoromethyl)-2,3-dihydrobenzofuran (4c)}

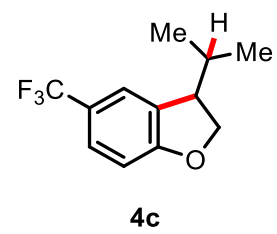

Synthesized according to GP F (Kessil lamp $427 \mathrm{~nm}$ ) from 2-bromo-1-((3-methylbut-2-en-1-yl)oxy)(trifluoromethyl)benzene (2c). Purification by flash chromatography $\left(\mathrm{SiO}_{2}, n\right.$-pentane:EtOAc $\left.=40: 1\right)$ afforded $\mathbf{4 c}$ (44 mg, $0.19 \mathrm{mmol}, 96 \%$ ) as a colourless liquid.

Analytical data of $4 c$ :

TLC $\left(\mathrm{SiO}_{2}\right): \mathrm{R}_{f}(n$-pentane:EtOAc $=40: 1)=0.36$

${ }^{1} \mathbf{H}$ NMR $\left(400 \mathrm{MHz}, \mathrm{CDCl}_{3}\right): \delta=7.44-7.36(\mathrm{~m}, 2 \mathrm{H}), 6.85-6.78(\mathrm{~m}, 1 \mathrm{H}), 4.60(\mathrm{t}, J=9.3 \mathrm{~Hz}, 1 \mathrm{H}), 4.46(\mathrm{dd}, J=$ 9.2, 5.2 Hz, 1H), 3.37 (dt, $J=9.5,5.1 \mathrm{~Hz}, 1 \mathrm{H}), 1.99$ (heptd, $J=6.8,4.9 \mathrm{~Hz}, 1 \mathrm{H}), 0.96(\mathrm{~d}, J=6.8 \mathrm{~Hz}, 3 \mathrm{H}), 0.88$ (d, $J=6.8 \mathrm{~Hz}, 3 \mathrm{H}) \mathrm{ppm}$.

${ }^{13} \mathbf{C}$ NMR $\left(101 \mathrm{MHz}, \mathrm{CDCl}_{3}\right): \delta=163.2,130.5,126.3\left(\mathrm{q},{ }^{3} J_{\mathrm{FC}}=3.9 \mathrm{~Hz}\right), 124.8\left(\mathrm{q},{ }^{1} J_{\mathrm{FC}}=271.0 \mathrm{~Hz}\right), 122.7\left(\mathrm{q},{ }^{2} J_{\mathrm{FC}}\right.$ $=32.2 \mathrm{~Hz}), 122.4\left(\mathrm{q},{ }^{3} J_{\mathrm{FC}}=3.9 \mathrm{~Hz}\right), 109.5,74.8,47.8,31.8,19.8,18.5 \mathrm{ppm}$.

${ }^{19} \mathbf{F}$ NMR $\left(376 \mathrm{MHz}, \mathrm{CDCl}_{3}\right) \delta=-60.96 \mathrm{ppm}$.

IR (ATR): 2963 (w), 1619 (2), 1499 (w), 1317 (s), 1154 (s), 1108 (s), 1057 (m), 956 (w), 824 (m), 662 (w) cm'.

HRMS (APPI/LTQ-Orbitrap): calcd. for $\left[\mathrm{C}_{12} \mathrm{H}_{13} \mathrm{~F}_{3} \mathrm{O}\right]^{+},[\mathrm{M}]^{+}: 230.0913$; found: 230.0913. 


\section{3-Isopropyl-2,3-dihydrobenzofuran-4-ol (4d)}

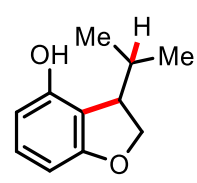

4d

Synthesized according to GP F (Kessil lamp $427 \mathrm{~nm}$ ) from 2-bromo-3-((3-methylbut-2-en-1-yl)oxy)phenol (2d) (46.0 mg, $200 \mu$ mol, 1.00 equiv.). Purification by flash chromatography $\left(\mathrm{SiO}_{2}, n\right.$-pentane:EtOAc $\left.=20: 1\right)$ afforded 4d (21 mg, $118 \mu \mathrm{mol}, 66 \%)$ as a colourless gum.

Analytical data of $\mathbf{4 d}$ :

TLC $\left(\mathrm{SiO}_{2}\right): \mathrm{R}_{f}(n$-pentane:EtOAc $=20: 1)=0.12$

${ }^{1}$ H NMR $\left(400 \mathrm{MHz}, \mathrm{CDCl}_{3}\right): \delta=6.99(\mathrm{td}, J=8.0,0.6 \mathrm{~Hz}, 1 \mathrm{H}), 6.43-6.36(\mathrm{~m}, 1 \mathrm{H}), 6.28(\mathrm{dd}, J=8.1,0.8 \mathrm{~Hz}, 1 \mathrm{H})$, $4.67(\mathrm{~s}, 1 \mathrm{H}), 4.48-4.41(\mathrm{~m}, 2 \mathrm{H}), 3.54-3.45(\mathrm{~m}, 1 \mathrm{H}), 2.25$ (heptd, $J=6.9,4.1 \mathrm{~Hz}, 1 \mathrm{H}), 0.97(\mathrm{~d}, J=6.9 \mathrm{~Hz}, 3 \mathrm{H})$, $0.81(\mathrm{~d}, J=6.8 \mathrm{~Hz}, 3 \mathrm{H}) \mathrm{ppm}$.

${ }^{13}$ C NMR (101 MHz, $\left.\mathrm{CDCl}_{3}\right): \delta=162.2,153.0,129.4,115.3,107.9,102.6,73.1,46.5,29.9,20.8,17.3 \mathrm{ppm}$.

IR (ATR): 3401 (br), 2958 (m), 1611 (m), 1458 (s), 1280 (m), 1014 (s), 949 (w), 784 (w) cm'.

HRMS (ESI): calcd. for $\left[\mathrm{C}_{11} \mathrm{H}_{14} \mathrm{O}_{2}+\mathrm{H}\right]^{+},[\mathrm{M}+\mathrm{H}]^{+}:$179.1067; found: 179.1061 .

\section{3-Isopropyl-4-((3-methylbut-2-en-1-yl)oxy)-2,3-dihydrobenzofuran (4e)}

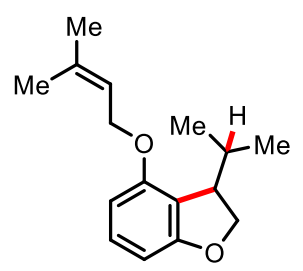

$4 \mathrm{e}$

Synthesized according to GP F (Kessil lamp $427 \mathrm{~nm}$ ) from 2-bromo-1,3-bis((3-methylbut-2-en-1-yl)oxy)benzene (2e) $\left(65.1 \mathrm{mg}, 200 \mu \mathrm{mol}, 1.00\right.$ equiv.). Purification by flash chromatography $\left(\mathrm{SiO}_{2}, n\right.$-pentane:EtOAc $\left.=40: 1\right)$ afforded $4 \mathbf{e}(31 \mathrm{mg}, 126 \mu \mathrm{mol}, 63 \%)$ as a colourless oil.

Analytical data of $4 \boldsymbol{e}$ :

TLC $\left(\mathrm{SiO}_{2}\right): \mathrm{R}_{f}(n$-pentane:EtOAc $=40: 1)=0.26$

${ }^{1} \mathbf{H}$ NMR (400 MHz, CDCl $): \delta=7.10-7.02(\mathrm{~m}, 1 \mathrm{H}), 6.42(\mathrm{dd}, J=8.1,3.6 \mathrm{~Hz}, 2 \mathrm{H}), 5.51-5.42(\mathrm{~m}, 1 \mathrm{H}), 4.61-$ $4.48(\mathrm{~m}, 2 \mathrm{H}), 4.46-4.39(\mathrm{~m}, 2 \mathrm{H}), 3.56-3.47(\mathrm{~m}, 1 \mathrm{H}), 2.31$ (heptd, $J=6.9,3.9 \mathrm{~Hz}, 1 \mathrm{H}), 1.79(\mathrm{~s}, 3 \mathrm{H}), 1.73(\mathrm{~s}, 3 \mathrm{H})$, $0.95(\mathrm{~d}, J=6.9 \mathrm{~Hz}, 3 \mathrm{H}), 0.75(\mathrm{~d}, J=6.9 \mathrm{~Hz}, 3 \mathrm{H}) \mathrm{ppm}$.

${ }^{13} \mathbf{C}$ NMR $\left(101 \mathrm{MHz}, \mathrm{CDCl}_{3}\right): \delta=161.7,156.5,137.3,129.1,120.3,117.0,104.3,102.7,72.8,65.0,46.8,29.2$, 25.9, 20.9, 18.4, $17.0 \mathrm{ppm}$.

IR (ATR): 2957 (m), 1605 (m), 1453 (m), 1269 (m), 1252 (m), 1058 (s), 782 (w), 745 (w) cm-1.

HRMS (ESI): calcd. for $\left[\mathrm{C}_{16} \mathrm{H}_{22} \mathrm{O}_{2}+\mathrm{H}\right]^{+},[\mathrm{M}+\mathrm{H}]^{+}: 247.1693$; found: 247.1697. 


\section{3-Isopropyl-2,3-dihydrobenzofuran-5-carboxylic acid (4f)}

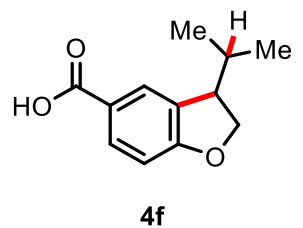

Synthesized according to GP F (Kessil lamp $427 \mathrm{~nm}$ ) from 3-bromo-4-((3-methylbut-2-en-1-yl)oxy)benzoic acid (2f) $(57.0 \mathrm{mg}, 200 \mu \mathrm{mol}, 1.00$ equiv.) and HBpin (72.5 $\mu \mathrm{L}, 64.0 \mathrm{mg}, 500 \mu \mathrm{mol}, 2.50$ equiv.). After $16 \mathrm{~h}$, all volatiles were remove under reduced pressure and the residue was washed with $n$-pentane to afford $\mathbf{4 f}$ ( $35 \mathrm{mg}$, $170 \mu \mathrm{mol}, 85 \%)$ as a white solid.

Analytical data of $4 \mathrm{f}$ :

TLC $\left(\mathrm{SiO}_{2}\right): \mathrm{R}_{f}\left(n\right.$-pentane: $\left.\mathrm{Et}_{2} \mathrm{O}=1: 1\right)=0.40$

Mp: $142.1-143.7^{\circ} \mathrm{C}$

${ }^{1} \mathbf{H}$ NMR $\left(400 \mathrm{MHz}, \mathrm{CDCl}_{3}\right): \delta=12.28($ br s, $1 \mathrm{H}), 8.03-7.89(\mathrm{~m}, 2 \mathrm{H}), 6.81(\mathrm{~d}, J=8.4 \mathrm{~Hz}, 1 \mathrm{H}), 4.63(\mathrm{t}, J=9.3$ $\mathrm{Hz}, 1 \mathrm{H}), 4.48(\mathrm{dd}, J=9.3,5.2 \mathrm{~Hz}, 1 \mathrm{H}), 3.44-3.34(\mathrm{~m}, 1 \mathrm{H}), 2.05-1.97(\mathrm{~m}, 1 \mathrm{H}), 0.97(\mathrm{~d}, J=6.8 \mathrm{~Hz}, 3 \mathrm{H}), 0.89(\mathrm{~d}$, $J=6.8 \mathrm{~Hz}, 3 \mathrm{H}) \mathrm{ppm}$.

${ }^{13} \mathrm{C}$ NMR $\left(101 \mathrm{MHz}, \mathrm{CDCl}_{3}\right): \delta=172.1,165.5,132.3,130.3,127.7,121.6,109.4,75.2,47.5,31.8,19.8,18.3 \mathrm{ppm}$. IR (ATR): 3641 (w), 2967 (m), 1678 (s), 1607 (m), 1411 (m), 1296 (m), 1245 (m), 1052 (w), 952 (w) cm-1. HRMS (ESI): calcd. for $\left[\mathrm{C}_{12} \mathrm{H}_{14} \mathrm{O}_{3}+\mathrm{H}\right]^{+},[\mathrm{M}+\mathrm{H}]^{+}:$207.1016; found: 207.1010.

\section{Methyl 3-isopropyl-2,3-dihydrobenzofuran-5-carboxylate (4g)}

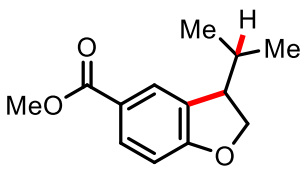

$\mathbf{4 g}$

Synthesized according to GP F (Kessil lamp $427 \mathrm{~nm}$ ) from methyl 3-bromo-4-((3-methylbut-2-en-1yl)oxy)benzoate $(\mathbf{2 g}) \quad(59.8 \mathrm{mg}, 200 \mu \mathrm{mol}, 1.00$ equiv. $)$. Purification by flash chromatography $\left(\mathrm{SiO}_{2}\right.$, $n$-pentane:EtOAc $=40: 1)$ afforded $\mathbf{4 g}(37 \mathrm{mg}, 168 \mu \mathrm{mol}, 84 \%)$ as a white solid

Analytical data of $\mathbf{4 g}$ :

TLC $\left(\mathrm{SiO}_{2}\right): \mathrm{R}_{f}(n$-pentane:EtOAc $=40: 1)=0.19$

Mp: $50.2-52.1{ }^{\circ} \mathrm{C}$.

${ }^{1} \mathbf{H}$ NMR (400 MHz, CDCl$): \delta=7.90-7.85(\mathrm{~m}, 2 \mathrm{H}), 6.79-6.75(\mathrm{~m}, 1 \mathrm{H}), 4.59(\mathrm{t}, J=9.3 \mathrm{~Hz}, 1 \mathrm{H}), 4.45(\mathrm{dd}, J=$ 9.2, $5.2 \mathrm{~Hz}, 1 \mathrm{H}), 3.87$ (s, 3H), $3.40-3.33(\mathrm{~m}, 1 \mathrm{H}), 1.99$ (heptd, $J=6.8,4.9 \mathrm{~Hz}, 1 \mathrm{H}), 0.95(\mathrm{~d}, J=6.8 \mathrm{~Hz}, 3 \mathrm{H}), 0.86$ (d, $J=6.8 \mathrm{~Hz}, 3 \mathrm{H}) \mathrm{ppm}$.

${ }^{13} \mathbf{C}$ NMR $\left(101 \mathrm{MHz}, \mathrm{CDCl}_{3}\right): \delta=167.2,164.7,131.4,130.0,127.0,122.5,109.2,75.0,51.9,47.6,31.7,19.8$, $18.3 \mathrm{ppm}$.

IR (ATR): 2957 (m), 1710 (s), 1608 (m), 1491 (m), 1437 (m), 1256 (s), 1157 (m), 1108 (s), 952 (s), 834 (w), 767 (m), $667(\mathrm{w}) \mathrm{cm}^{-1}$. 
HRMS (ESI): calcd. for $\left[\mathrm{C}_{13} \mathrm{H}_{16} \mathrm{O}_{3}+\mathrm{H}\right]^{+},[\mathrm{M}+\mathrm{H}]^{+}:$221.1172; found: 221.1142 .

7-Chloro-3-isopropyl-2,3-dihydrobenzofuran (4h)

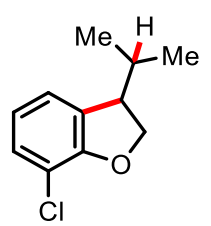

4h

Synthesized according to GP F (Kessil lamp $427 \mathrm{~nm}$ ) from 1-bromo-3-chloro-2-((3-methylbut-2-en-1yl)oxy)benzene (2h) $\left(55.1 \mathrm{mg}, 200 \mu \mathrm{mol}, 1.00\right.$ equiv.). Purification by flash chromatography $\left(\mathrm{SiO}_{2}\right.$, $n$-pentane:EtOAc = 1:0 - 40:1) afforded $\mathbf{4 h}(32 \mathrm{mg}, 162 \mu \mathrm{mol}, 81 \%)$ as a colourless oil.

Analytical data of $\mathbf{4 h}$ :

TLC $\left(\mathrm{SiO}_{2}\right): \mathrm{R}_{f}(n$-pentane:EtOAc $=40: 1)=0.36$

${ }^{1} \mathbf{H}$ NMR $\left(400 \mathrm{MHz}, \mathrm{CDCl}_{3}\right): \delta=7.13(\mathrm{~d}, J=7.9 \mathrm{~Hz}, 1 \mathrm{H}), 7.06(\mathrm{~d}, J=7.4 \mathrm{~Hz}, 1 \mathrm{H}), 6.79(\mathrm{dd}, J=8.0,7.4 \mathrm{~Hz}, 1 \mathrm{H})$, $4.62(\mathrm{t}, J=9.2 \mathrm{~Hz}, 1 \mathrm{H}), 4.48(\mathrm{dd}, J=9.2,5.3 \mathrm{~Hz}, 1 \mathrm{H}), 3.41(\mathrm{dtt}, J=9.3,5.3,0.9 \mathrm{~Hz}, 1 \mathrm{H}), 1.98$ (heptd, $J=6.9,5.0$ $\mathrm{Hz}, 1 \mathrm{H}), 0.95(\mathrm{~d}, J=6.8 \mathrm{~Hz}, 3 \mathrm{H}), 0.88(\mathrm{~d}, J=6.9 \mathrm{~Hz}, 3 \mathrm{H}) \mathrm{ppm}$.

${ }^{13}$ C NMR (101 MHz, $\left.\mathrm{CDCl}_{3}\right): \delta=156.3,131.4,128.6,123.5,121.3,115.1,74.7,49.0,31.8,19.9,18.5$ ppm.

IR (ATR): 2961 (s), 2901 (m), 1605 (w), 1449 (s), 1220 (m), 1076 (m), 1055 (m), 955 (m), 889 (w) cm ${ }^{-1}$.

HRMS (APPI/LTQ-Orbitrap): calcd. for $\left[\mathrm{C}_{11} \mathrm{H}_{13} \mathrm{ClO}\right]^{+},[\mathrm{M}]^{+}:$196.0649; found: 196.0647.

\section{3-Isopropyl-2,3-dihydrofuro[3,2-b]pyridine (4i)}

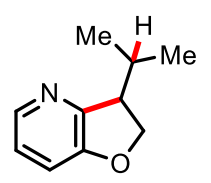

$4 \mathbf{i}$

Synthesized according to GP F (Kessil lamp $427 \mathrm{~nm}$ ) from 2-bromo-3-((3-methylbut-2-en-1-yl)oxy)pyridine (2i) (48.4 mg, $200 \mu \mathrm{mol}, 1.00$ equiv.). Purification by flash chromatography $\left(\mathrm{SiO}_{2}, n\right.$-pentane:EtOAc $\left.=3: 1\right)$ afforded $4 \mathbf{i}(20 \mathrm{mg}, 123 \mu \mathrm{mol}, 61 \%)$ as a colourless oil.

Analytical data of 4 :

TLC $\left(\mathrm{SiO}_{2}\right): \mathrm{R}_{f}(n$-pentane:EtOAc $=3: 1)=0.63$

${ }^{1} \mathbf{H}$ NMR $\left(400 \mathrm{MHz}, \mathrm{CDCl}_{3}\right): \delta=8.06(\mathrm{dd}, J=3.8,2.4 \mathrm{~Hz}, 1 \mathrm{H}), 7.02-6.97(\mathrm{~m}, 2 \mathrm{H}), 4.62(\mathrm{t}, J=9.4 \mathrm{~Hz}, 1 \mathrm{H}), 4.46$ (dd, $J=9.3,5.8 \mathrm{~Hz}, 1 \mathrm{H}), 3.43-3.34(\mathrm{~m}, 1 \mathrm{H}), 2.21$ (heptd, $J=6.9,4.8 \mathrm{~Hz}, 1 \mathrm{H}), 1.02(\mathrm{~d}, J=6.9 \mathrm{~Hz}, 3 \mathrm{H}), 0.86(\mathrm{~d}$, $J=6.8 \mathrm{~Hz}, 3 \mathrm{H}) \mathrm{ppm}$.

${ }^{13}$ C NMR (101 MHz, $\left.\mathrm{CDCl}_{3}\right): \delta=154.2,153.1,141.6,122.4,115.6,73.3,48.1,31.0,20.2,18.0$ ppm.

IR (ATR): 2958 (m), 1575 (w), 1426 (s), 1261 (m), 1157 (w), 1103 (w), 945 (s), 783 (m) cm-1.

HRMS (ESI): calcd. for $\left[\mathrm{C}_{10} \mathrm{H}_{13} \mathrm{NO}+\mathrm{H}\right]^{+},[\mathrm{M}+\mathrm{H}]^{+}:$164.1070; found: 164.1065 .

All spectroscopic data corresponded to the reported literature values. ${ }^{[31]}$ 


\section{7-Bromo-3-isopropyl-2,3-dihydrobenzofuran (4j)}

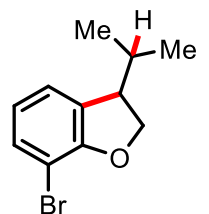

$4 \mathrm{j}$

Synthesized according to GP F (white LEDs) from 1-bromo-3-iodo-2-((3-methylbut-2-en-1-yl)oxy)benzene (1j) (70.4 mg, $191 \mu$ mol, 1.00 equiv.). Purification by flash chromatography $\left(\mathrm{SiO}_{2}, n\right.$-pentane:EtOAc $\left.=40: 1\right)$ afforded 4j (45 $\mathrm{mg}, 186 \mu \mathrm{mol}, 97 \%)$ as a colourless oil.

Analytical data of $4 \mathrm{j}$ :

TLC $\left(\mathrm{SiO}_{2}\right): \mathrm{R}_{f}(n$-pentane:EtOAc $=40: 1)=0.37$

${ }^{1} \mathbf{H}$ NMR $\left(400 \mathrm{MHz}, \mathrm{CDCl}_{3}\right): \delta=7.28(\mathrm{~d}, J=8.0 \mathrm{~Hz}, 1 \mathrm{H}), 7.10(\mathrm{~d}, J=7.4 \mathrm{~Hz}, 1 \mathrm{H}), 6.73(\mathrm{dd}, J=8.0,7.3 \mathrm{~Hz}, 1 \mathrm{H})$, $4.62(\mathrm{t}, J=9.2 \mathrm{~Hz}, 1 \mathrm{H}), 4.47(\mathrm{dd}, J=9.2,5.4 \mathrm{~Hz}, 1 \mathrm{H}), 3.44$ (dtt, $J=9.3,5.3,0.9 \mathrm{~Hz}, 1 \mathrm{H}), 1.98$ (heptd, $J=6.8,4.9$ $\mathrm{Hz}, 1 \mathrm{H}), 0.95(\mathrm{~d}, J=6.9 \mathrm{~Hz}, 3 \mathrm{H}), 0.88(\mathrm{~d}, J=6.8 \mathrm{~Hz}, 3 \mathrm{H}) \mathrm{ppm}$.

${ }^{13}$ C NMR (101 MHz, $\left.\mathrm{CDCl}_{3}\right): \delta=157.7,131.4,131.1,124.2,121.7,102.7,74.4,49.2,31.8,19.9,18.5$ ppm.

All spectroscopic data corresponded to the reported literature values. ${ }^{[32]}$

\section{3-Benzyl-2,3-dihydrobenzofuran (4k)}

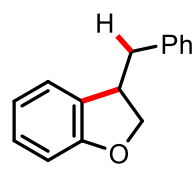

$4 \mathbf{k}$

Synthesized according to GP F (KESSIL lamp) from (E)-1-bromo-2-(cinnamyloxy)benzene (2k) (57.8 mg, $200 \mu \mathrm{mol}, 1.00$ equiv.). Purification by flash chromatography $\left(\mathrm{SiO}_{2}, n\right.$-pentane:EtOAc $\left.=40: 1\right)$ afforded $\mathbf{4 k}(36 \mathrm{mg}$, $171 \mu \mathrm{mol}, 86 \%)$ as a colourless oil.

Analytical data of $\mathbf{4 k}$ :

TLC $\left(\mathrm{SiO}_{2}\right): \mathrm{R}_{f}(n$-pentane:EtOAc $=40: 1)=0.26$

${ }^{1} \mathbf{H}$ NMR $\left(400 \mathrm{MHz}, \mathrm{CDCl}_{3}\right): \delta=7.37-7.28(\mathrm{~m}, 2 \mathrm{H}), 7.30-7.21(\mathrm{~m}, 1 \mathrm{H}), 7.24-7.17(\mathrm{~m}, 2 \mathrm{H}), 7.19-7.10(\mathrm{~m}$, $1 \mathrm{H}), 7.02-6.95(\mathrm{~m}, 1 \mathrm{H}), 6.87-6.78(\mathrm{~m}, 2 \mathrm{H}), 4.54(\mathrm{t}, J=8.9 \mathrm{~Hz}, 1 \mathrm{H}), 4.30(\mathrm{dd}, J=8.9,6.0 \mathrm{~Hz}, 1 \mathrm{H}), 3.77(\mathrm{tt}, J=$ 8.9, $6.1 \mathrm{~Hz}, 1 \mathrm{H}), 3.08(\mathrm{dd}, J=13.8,6.4 \mathrm{~Hz}, 1 \mathrm{H}), 2.86(\mathrm{dd}, J=13.8,8.9 \mathrm{~Hz}, 1 \mathrm{H}) \mathrm{ppm}$.

${ }^{13}$ C NMR (101 MHz, $\left.\mathrm{CDCl}_{3}\right): \delta=160.1,139.3,130.4,129.1,128.7,128.5,126.6,124.7,120.4,109.8,76.5,43.6$, $41.2 \mathrm{ppm}$.

All spectroscopic data corresponded to the reported literature values. ${ }^{[30]}$ 


\section{3,3-Dimethyl-2,3-dihydrobenzofuran (4I)}

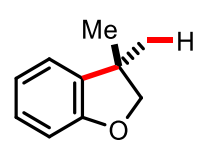

41

Synthesized according to GP F (Kessil lamp $427 \mathrm{~nm}$ ) from 1-bromo-2-((2-methylallyl)oxy)benzene (2l) (45.4 mg, $200 \mu \mathrm{mol}, 1.00$ equiv. $)$. Purification by flash chromatography $\left(\mathrm{SiO}_{2}, n\right.$-pentane:EtOAc $\left.=40: 1\right)$ afforded $\mathbf{4 l}(8 \mathrm{mg}$, $54.0 \mu \mathrm{mol}, 27 \%$ ) as a colourless oil.

Analytical data of 4 :

TLC $\left(\mathrm{SiO}_{2}\right): \mathrm{R}_{f}(n$-pentane:EtOAc $=40: 1)=0.52$

${ }^{1} \mathbf{H}$ NMR (400 MHz, $\left.\mathrm{CDCl}_{3}\right): \delta=7.17-7.07(\mathrm{~m}, 2 \mathrm{H}), 6.93-6.84(\mathrm{~m}, 1 \mathrm{H}), 6.83-6.76(\mathrm{~m}, 1 \mathrm{H}), 4.23(\mathrm{~s}, 2 \mathrm{H}), 1.35$ (s, 6H) ppm.

${ }^{13}$ C NMR (101 MHz, $\left.\mathrm{CDCl}_{3}\right): \delta=159.3,136.7,128.0,122.4,120.7,109.8,84.5,42.0,27.7$ (2C) ppm.

All spectroscopic data corresponded to the reported literature values. ${ }^{[33]}$

\section{$2 H$-spiro[benzofuran-3,1'-cyclohexane] $(4 \mathrm{~m})$}

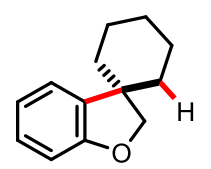

$4 \mathrm{~m}$

Synthesized according to GP F (Kessil lamp 427 nm) from 1-bromo-2-(cyclohex-1-en-1-ylmethoxy)benzene (2m) (51.0 mg, $200 \mu \mathrm{mol}, 1.00$ equiv.). Purification by flash chromatography $\left(\mathrm{SiO}_{2}, n\right.$-pentane:EtOAc $\left.=1: 0-40: 1\right)$ afforded $\mathbf{4 m}(9 \mathrm{mg}, 47 \mu \mathrm{mol}, 24 \%)$ as a colourless liquid.

Analytical data of $\mathbf{4 m}$ :

TLC $\left(\mathrm{SiO}_{2}\right): \mathrm{R}_{f}(n$-pentane:EtOAc $=40: 1)=0.65$

${ }^{1} \mathbf{H}$ NMR $\left(400 \mathrm{MHz}, \mathrm{CDCl}_{3}\right): \delta=7.16-7.07(\mathrm{~m}, 2 \mathrm{H}), 6.87(\mathrm{td}, J=7.4,1.0 \mathrm{~Hz}, 1 \mathrm{H}), 6.82-6.75(\mathrm{~m}, 1 \mathrm{H}), 4.36(\mathrm{~s}$, $2 \mathrm{H}), 1.82-1.58(\mathrm{~m}, 7 \mathrm{H}), 1.40-1.31(\mathrm{~m}, 3 \mathrm{H}) \mathrm{ppm}$.

${ }^{13} \mathrm{C}$ NMR (101 MHz, $\left.\mathrm{CDCl}_{3}\right): \delta=159.4,136.4,128.2,123.0,120.5,109.8,81.1,46.3,36.9$ (2C), 25.6, 23.5 (2C) ppm.

IR (ATR): 2925 (s), 2852 (m), 1599 (w), 1479 (s), 1449 (m), 1228 (s), 977 (s) 749 (s) cm².

HRMS (ESI): calcd. for $\left[\mathrm{C}_{13} \mathrm{H}_{16} \mathrm{O}\right]^{+},[\mathrm{M}]^{+}:$188.1196; found: 188.1194 .

3-(6-Methylhept-5-en-2-yl)-2,3-dihydrobenzofuran (4n)

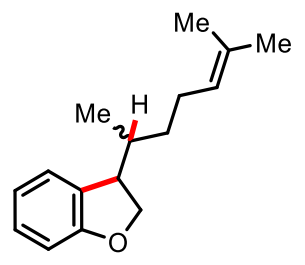

$4 n$ 
Synthesized according to GP F (Kessil lamp $427 \mathrm{~nm}$ ) from (E)-1-bromo-2-((3,7-dimethylocta-2,6-dien-1yl)oxy)benzene (2n) $\left(61.9 \mathrm{mg}, \quad 200 \mu \mathrm{mol}, 1.00\right.$ equiv.). Purification by flash chromatography $\left(\mathrm{SiO}_{2}\right.$, $n$-pentane:EtOAc $=40: 1)$ afforded $\mathbf{4 n}(40 \mathrm{mg}, 173 \mu \mathrm{mol}, 87 \%)$ as a colourless oil $($ d.r. $=50: 50)$.

Analytical data of $\mathbf{4 n}$ :

TLC $\left(\mathrm{SiO}_{2}\right): \mathrm{R}_{f}(n$-pentane:EtOAc $=40: 1)=0.41$

${ }^{1}$ H NMR $\left(400 \mathrm{MHz}, \mathrm{CDCl}_{3}\right): \delta=7.20-7.07(\mathrm{~m}, 2 \mathrm{H}), 6.89-6.80(\mathrm{~m}, 1 \mathrm{H}), 6.77(\mathrm{~d}, J=8.0 \mathrm{~Hz}, 1 \mathrm{H}), 5.15-5.01$ $(\mathrm{m}, 1 \mathrm{H}), 4.55(\mathrm{t}, J=9.2 \mathrm{~Hz}, 1 / 2 \mathrm{H}), 4.50(\mathrm{t}, J=9.3 \mathrm{~Hz}, 1 / 2 \mathrm{H}), 4.36(\mathrm{ddd}, \mathrm{J}=9.0,6.9,5.4 \mathrm{~Hz}, 1 \mathrm{H}), 3.50(\mathrm{dt}, J=9.7$, $4.9 \mathrm{~Hz}, 1 / 2 \mathrm{H}), 3.42(\mathrm{dt}, J=9.5,4.8 \mathrm{~Hz}, 1 / 2 \mathrm{H}), 2.14-1.73(\mathrm{~m}, 3 \mathrm{H}), 1.70(\mathrm{~s}, 3 / 2 \mathrm{H}), 1.68(\mathrm{~s}, 3 / 2 \mathrm{H}), 1.62(\mathrm{~s}, 3 / 2 \mathrm{H})$, $1.59(\mathrm{~s}, 3 / 2 \mathrm{H}), 1.51-1.31(\mathrm{~m}, 1 \mathrm{H}), 1.32-1.14(\mathrm{~m}, 1 \mathrm{H}), 0.89(\mathrm{~d}, \mathrm{~J}=6.8 \mathrm{~Hz}, 3 / 2 \mathrm{H}), 0.81(\mathrm{~d}, \mathrm{~J}=6.9 \mathrm{~Hz}, 3 / 2 \mathrm{H}) \mathrm{ppm}$. ${ }^{13} \mathrm{C}$ NMR $\left(101 \mathrm{MHz}, \mathrm{CDCl}_{3}\right): \delta=160.77,160.52,131.84,131.78,129.85,128.98,128.27,128.19,125.40,124.78$, $124.50,124.41,120.31,120.17,109.47,109.42,74.86$, 73.04, 47.29, 46.75, 36.54, 36.03, 34.73, 33.31, 25.98, 25.92, 25.89, 25.85, 17.86, 17.82, 16.44, $14.94 \mathrm{ppm}$.

IR (ATR): 2963 (m), 2914 (m), 1595 (m), 1483 (s), 1459 (m), 1229 (s), 958 (w), 749 (s) cm².

HRMS (ESI): calcd. for $\left[\mathrm{C}_{16} \mathrm{H}_{22} \mathrm{O}+\mathrm{H}\right]^{+},[\mathrm{M}+\mathrm{H}]^{+}: 231.1743$; found: 231.1738

\section{4-Isopropylchromane (4o)}

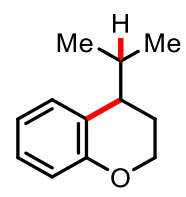

40

Synthesized according to GP F (Kessil lamp $427 \mathrm{~nm}$ ) from 1-bromo-2-((4-methylpent-3-en-1-yl)oxy)benzene (2o) (51.0 mg, $200 \mu \mathrm{mol}, 1.00$ equiv.). Purification by flash chromatography $\left(\mathrm{SiO}_{2}, n\right.$-pentane:EtOAc $\left.=40: 1\right)$ afforded 4o (16 mg, $91 \mu \mathrm{mol}, 45 \%)$ as a colourless liquid.

Analytical data of $\mathbf{4 o}$ :

TLC $\left(\mathrm{SiO}_{2}\right): \mathrm{R}_{f}(n$-pentane:EtOAc $=1: 0-40: 1)=0.47$

${ }^{1} \mathbf{H}$ NMR $\left(400 \mathrm{MHz}, \mathrm{CDCl}_{3}\right): \delta=7.19-7.12(\mathrm{~m}, 1 \mathrm{H}), 7.13-7.04(\mathrm{~m}, 1 \mathrm{H}), 6.86(\mathrm{td}, J=7.5,1.4 \mathrm{~Hz}, 1 \mathrm{H}), 6.80(\mathrm{dd}$, $J=8.1,1.3 \mathrm{~Hz}, 1 \mathrm{H}), 4.27(\mathrm{ddd}, J=10.7,6.1,4.5 \mathrm{~Hz}, 1 \mathrm{H}), 4.15-4.08(\mathrm{~m}, 1 \mathrm{H}), 2.73(\mathrm{q}, J=6.5 \mathrm{~Hz}, 1 \mathrm{H}), 2.32-$ $2.14(\mathrm{~m}, 1 \mathrm{H}), 1.92(\mathrm{tdd}, J=6.2,4.3,2.2 \mathrm{~Hz}, 2 \mathrm{H}), 1.06(\mathrm{~d}, J=6.8 \mathrm{~Hz}, 3 \mathrm{H}), 0.80(\mathrm{~d}, J=6.8 \mathrm{~Hz}, 3 \mathrm{H}) \mathrm{ppm}$.

${ }^{13}$ C NMR (101 MHz, $\left.\mathrm{CDCl}_{3}\right): \delta=155.2,129.0,127.2,125.8,120.1,116.9,65.0,39.8,30.7,23.0,21.2,17.6$ ppm. IR (ATR): 2958 (s), 2871 (m), 1580 (w), 1488 (s), 1451 (m), 1223 (s), 1068 (s), 752 (s) cm-1.

HRMS (ESI): calcd. for $\left[\mathrm{C}_{12} \mathrm{H}_{16} \mathrm{O}\right]^{+},[\mathrm{M}]^{+}:$176.1196; found: 176.1195 .

\section{1-(3-Isopropylindolin-1-yl)ethan-1-one (4p)}

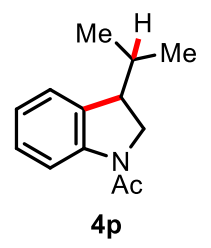


Synthesized according to GP F (Kessil lamp 427) from $N$-(2-bromophenyl)- $N$-(3-methylbut-2-en-1yl)acetamide (2p) $\left(56.4 \mathrm{mg}, 200 \mu \mathrm{mol}, 1.00\right.$ equiv.). Purification by flash chromatography $\left(\mathrm{SiO}_{2}, n\right.$-pentane:EtOAc $=1: 1)$ afforded $4 \mathbf{p}(37 \mathrm{mg}, 182 \mu \mathrm{mol}, 91 \%)$ as a colourless oil.

Analytical data of $4 p$ :

TLC $\left(\mathrm{SiO}_{2}\right): \mathrm{R}_{f}(n$-pentane:EtOAc $=1: 1)=0.59$

${ }^{1} \mathbf{H}$ NMR $\left(400 \mathrm{MHz}, \mathrm{CDCl}_{3}\right): \delta=8.20(\mathrm{~d}, J=8.1 \mathrm{~Hz}, 1 \mathrm{H}), 7.25-7.13(\mathrm{~m}, 2 \mathrm{H}), 7.02(\mathrm{td}, J=7.5,1.1 \mathrm{~Hz}, 1 \mathrm{H}), 4.00$ (t, $J=10.2 \mathrm{~Hz}, 1 \mathrm{H}), 3.79$ (dd, $J=10.6,5.0 \mathrm{~Hz}, 1 \mathrm{H}), 3.35(\mathrm{dt}, J=9.6,4.7 \mathrm{~Hz}, 1 \mathrm{H}), 2.24(\mathrm{~s}, 3 \mathrm{H}), 2.13-1.99(\mathrm{~m}$, $1 \mathrm{H}), 0.98(\mathrm{~d}, J=6.9 \mathrm{~Hz}, 3 \mathrm{H}), 0.80(\mathrm{~d}, J=6.9 \mathrm{~Hz}, 3 \mathrm{H}) \mathrm{ppm}$.

${ }^{13} \mathrm{C}$ NMR (101 MHz, $\left.\mathrm{CDCl}_{3}\right): \delta=168.7,143.3,133.9,128.0,124.5,123.6,116.9,51.3,46.1,32.0,24.4,20.2$, $17.5 \mathrm{ppm}$.

All spectroscopic data corresponded to the reported literature values. ${ }^{[34]}$

\section{3-Isopropyl-1-(3-methylbut-2-en-1-yl)indoline (4q)}

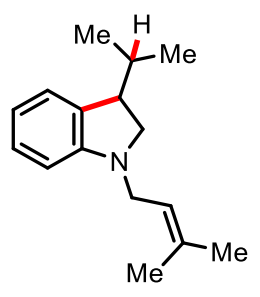

$4 q$

Synthesized according to GP F (Kessil lamp 427) from 2-bromo- $N, N$-bis(3-methylbut-2-en-1-yl)aniline (2q) (61.7 mg, $200 \mu$ mol, 1.00 equiv.). Purification by flash chromatography $\left(\mathrm{SiO}_{2}, n\right.$-pentane:EtOAc $\left.=40: 1\right)$ afforded 4q (44 mg, $191 \mu \mathrm{mol}, 96 \%$ ) as a colourless liquid.

Analytical data of $\mathbf{4 q}$ :

TLC $\left(\mathrm{SiO}_{2}\right): \mathrm{R}_{f}(n$-pentane:EtOAc $=40: 1)=0.32$

${ }^{1} \mathrm{H}$ NMR $\left(400 \mathrm{MHz}, \mathrm{CDCl}_{3}\right): \delta=7.10-7.02(\mathrm{~m}, 2 \mathrm{H}), 6.64(\mathrm{td}, J=7.4,1.0 \mathrm{~Hz}, 1 \mathrm{H}), 6.48(\mathrm{dd}, J=8.2,1.0 \mathrm{~Hz}, 1 \mathrm{H})$, $5.32-5.23(\mathrm{~m}, 1 \mathrm{H}), 3.71-3.64(\mathrm{~m}, 2 \mathrm{H}), 3.41-3.29(\mathrm{~m}, 1 \mathrm{H}), 3.19-3.05(\mathrm{~m}, 2 \mathrm{H}), 2.10-1.96(\mathrm{~m}, 1 \mathrm{H}), 1.75(\mathrm{~s}$, $3 \mathrm{H}), 1.72(\mathrm{~s}, 3 \mathrm{H}), 0.99(\mathrm{~d}, J=6.8 \mathrm{~Hz}, 3 \mathrm{H}), 0.88(\mathrm{~d}, J=6.8 \mathrm{~Hz}, 3 \mathrm{H}) \mathrm{ppm}$.

${ }^{13}$ C NMR (101 MHz, $\left.\mathrm{CDCl}_{3}\right): \delta=152.9,135.3,132.9,127.5,124.6,120.4,117.1,107.2,55.2,47.0,46.6,30.8$, 25.9, 20.7, 18.9, $18.1 \mathrm{ppm}$.

All spectroscopic data corresponded to the reported literature values. ${ }^{[35]}$

1,5-Diisopropyl-1,2,4,5-tetrahydropyrrolo[3,2,1-hi]indole (4r)

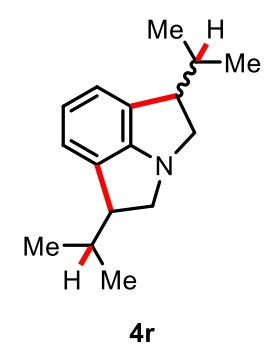

Synthesized according to GP F (Kessil lamp $427 \mathrm{~nm}$ ) from 2,6-dibromo- $N, N$-bis(3-methylbut-2-en-1-yl)aniline (2r) (77.4 mg, $200 \mu \mathrm{mol}, 1.00$ equiv.), DBU (59.7 $\mu \mathrm{L}, 60.9 \mathrm{mg}, 400 \mu \mathrm{mol}, 2.00$ equiv.), SPO ( $86.6 \mu \mathrm{L}, 50 \mathrm{mg} / \mathrm{mL}$ 
in MeCN stock solution, $4.33 \mathrm{mg}, 20 \mu \mathrm{mol}, 10 \mathrm{~mol} \%)$ and HBpin (63.8 $\mu \mathrm{L} .56 .3 \mathrm{mg}, 440 \mu \mathrm{mol}, 2.20$ equiv.). Purification by flash chromatography $\left(\mathrm{SiO}_{2}, n\right.$-pentane:EtOAc $\left.=40: 1-20: 1\right)$ afforded $\mathbf{4 r}(13 \mathrm{mg}, 56 \mu \mathrm{mol}, 28 \%)$ as a faint yellow oil $($ d.r. $=1: 1)$.

Analytical data of $4 r$ :

TLC $\left(\mathrm{SiO}_{2}\right): \mathrm{R}_{f}(n$-pentane:EtOAc $=40: 1)=0.22$

${ }^{1} \mathbf{H}$ NMR $\left(400 \mathrm{MHz}, \mathrm{CDCl}_{3}\right): \delta=6.96(\mathrm{~d}, J=7.3 \mathrm{~Hz}, 2 \mathrm{H}), 6.63(\mathrm{td}, J=7.3,2.3 \mathrm{~Hz}, 1 \mathrm{H}), 3.44-3.21(\mathrm{~m}, 4 \mathrm{H}), 3.03$ (dd, $J=8.6,6.5 \mathrm{~Hz}, 1 \mathrm{H}), 2.92(\mathrm{dd}, J=8.4,7.0 \mathrm{~Hz}, 1 \mathrm{H}), 1.99(\mathrm{dpd}, J=13.5,6.8,2.4 \mathrm{~Hz}, 2 \mathrm{H}), 1.08(\mathrm{~d}, J=4.3 \mathrm{~Hz}$, $3 \mathrm{H}), 1.07(\mathrm{~d}, J=4.3 \mathrm{~Hz}, 3 \mathrm{H}), 0.99(\mathrm{~d}, J=5.0 \mathrm{~Hz}, 3 \mathrm{H}), 0.97$ (d, $J=5.0 \mathrm{~Hz}, 3 \mathrm{H}) \mathrm{ppm}$.

${ }^{13} \mathrm{C} \mathrm{NMR}\left(101 \mathrm{MHz}, \mathrm{CDCl}_{3}\right): \delta=164.6,126.1,126.0,123.0,122.9,119.6,62.0,61.9,55.6,55.5,30.9,30.9,21.0$, 20.9, 20.9, $20.8 \mathrm{ppm}$.

IR (ATR): 2956 (s), 2869 (m), 2818 (w), 1585 (m), 1487 (m), 1450 (m), 1047 (w), 749 (s) cm-1.

HRMS (ESI): calcd. for $\left[\mathrm{C}_{16} \mathrm{H}_{23} \mathrm{~N}+\mathrm{H}\right]^{+},[\mathrm{M}+\mathrm{H}]^{+}:$230.1903; found: 230.1909.

\section{3-Isopropylhexahydro-4H-furo[2,3-b]pyran (4s)}

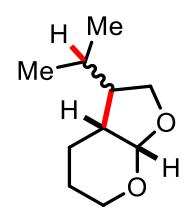

4s

Synthesized according to GP F (white LEDs) from 3-iodo-2-((3-methylbut-2-en-1-yl)oxy)tetrahydro-2H-pyran (1s) $\left(59.2 \mathrm{mg}, 200 \mu \mathrm{mol}, 1.00\right.$ equiv.). Purification by flash chromatography $\left(\mathrm{SiO}_{2}, n\right.$-pentane:EtOAc $\left.=10: 1\right)$ afforded $4 \mathrm{~s}(22.0 \mathrm{mg}, 129 \mu \mathrm{mol}, 65 \%)$ as a colourless oil (cis/trans $=64: 36)$.

Synthesized according to GP F (Kessil lamp $427 \mathrm{~nm}$ ) from 3-bromo-2-((3-methylbut-2-en-1-yl)oxy)tetrahydro2H-pyran (2s) (49.8 mg, $200 \mu \mathrm{mol}, 1.00$ equiv.). Purification by flash chromatography $\left(\mathrm{SiO}_{2}, n\right.$-pentane:EtOAc $=$ 10:1) afforded $4 \mathbf{s}(20.0 \mathrm{mg}, 118 \mu \mathrm{mol}, 59 \%)$ as a colourless oil ( cis/trans $\left.^{[36]}=64: 36\right)$.

Analytical data of $4 \mathrm{~s}$ :

TLC $\left(\mathrm{SiO}_{2}\right): \mathrm{R}_{f}(n$-pentane:EtOAc $=3: 1)=0.58$

${ }^{1}$ H NMR (400 MHz, CDCl $)$ ): cis: $\delta=5.29(\mathrm{~d}, J=3.2 \mathrm{~Hz}, 1 \mathrm{H}), 3.94(\mathrm{t}, J=7.8 \mathrm{~Hz}, 1 \mathrm{H}), 3.71-3.62(\mathrm{~m}, 3 \mathrm{H}), 1.96$ $-1.82(\mathrm{~m}, 2 \mathrm{H}), 1.71-1.54(\mathrm{~m}, 4 \mathrm{H}), 1.42-1.32(\mathrm{~m}, 1 \mathrm{H}), 0.93(\mathrm{~d}, J=6.5 \mathrm{~Hz}, 3 \mathrm{H}), 0.80(\mathrm{~d}, J=6.6 \mathrm{~Hz}, 3 \mathrm{H}) \mathrm{ppm}$. trans: $\delta=4.99(\mathrm{~d}, J=3.6 \mathrm{~Hz}, 1 \mathrm{H}), 4.18(\mathrm{t}, J=8.6 \mathrm{~Hz}, 1 \mathrm{H}), 3.93-3.83(\mathrm{~m}, 1 \mathrm{H}), 3.67-3.61(\mathrm{~m}, 1 \mathrm{H}), 3.46-3.39$ (m, 1H), $2.15-2.06(\mathrm{~m}, 1 \mathrm{H}), 1.90-1.76(\mathrm{~m}, 3 \mathrm{H}), 1.71-1.54(\mathrm{~m}, 2 \mathrm{H}), 1.42-1.32(\mathrm{~m}, 1 \mathrm{H}), 0.95(\mathrm{~d}, J=6.9 \mathrm{~Hz}$, $3 \mathrm{H}), 0.87(\mathrm{~d}, J=6.7 \mathrm{~Hz}, 3 \mathrm{H}) \mathrm{ppm}$.

${ }^{13} \mathrm{C}$ NMR (101 MHz, $\left.\mathrm{CDCl}_{3}\right)$ : cis: $\delta=102.3,69.2,60.9,49.1,35.9,26.5,23.5,21.9,21.2,19.0$ ppm. trans: $\delta=$ 102.7, 71.3, 64.5, 44.5, 41.5, 30.3, 23.7, 21.7, 20.9, $19.7 \mathrm{ppm}$.

All spectroscopic data corresponded to the reported literature values. ${ }^{[36-38]}$ 


\section{(Cyclopentylmethyl)benzene (4t)}

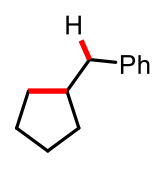

$\mathbf{4 t}$

Synthesized according to GP F (Kessil lamp $427 \mathrm{~nm}$ ) from [(E)-6-bromohex-1-enyl]benzene (47.8 mg, $200 \mu \mathrm{mol}$, 1.00 equiv.) 2t. Purification by flash chromatography ( $\mathrm{SiO} 2, \mathrm{n}$-pentane:EtOAc $=40: 1)$ afforded an inseparable mixture of $\mathbf{4 t}$ and $\mathbf{2 t}$. The mixture was analysed by qualitative NMR analysis with 1,3,5-trimethoxybenzene as internal standard to determine the NMR yield for $\mathbf{4 t}$ to be $39 \%$. The characteristic product signals matched those reported in literature. ${ }^{[39]}$

Analytical data of $\mathbf{4 t}$ :

${ }^{1}$ H NMR $\left(400 \mathrm{MHz}, \mathrm{CDCl}_{3}\right): \delta=7.30-7.24(\mathrm{~m}, 2 \mathrm{H}), 7.22-7.13(\mathrm{~m}, 3 \mathrm{H}), 2.61(\mathrm{~d}, J=7.4 \mathrm{~Hz}, 2 \mathrm{H}), 2.15-2.03$ $(\mathrm{m}, 1 \mathrm{H}), 1.75-1.67(\mathrm{~m}, 2 \mathrm{H}), 1.66-1.59(\mathrm{~m}, 2 \mathrm{H}), 1.59-1.45(\mathrm{~m}, 2 \mathrm{H}), 1.27-1.13(\mathrm{~m}, 2 \mathrm{H}) \mathrm{ppm}$.

\section{3-Benzyltetrahydrofuran $(4 \mathrm{u})$}

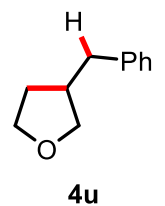

Synthesized according to GP F (Kessil lamp $427 \mathrm{~nm}$ ) from (E)-(3-(2-bromoethoxy)prop-1-en-1-yl)benzene (48.2 mg, $200 \mu$ mol, 1.00 equiv.) (2u). Purification by flash chromatography $\left(\mathrm{SiO}_{2}, n\right.$-pentane:EtOAc $\left.=20: 1\right)$ afforded $4 \mathbf{u}(12.0 \mathrm{mg}, 74.0 \mu \mathrm{mol}, 37 \%)$ as a colourless oil.

Analytical data of $\mathbf{4 u}$ :

TLC $\left(\mathrm{SiO}_{2}\right): \mathrm{R}_{f}(n$-pentane:EtOAc $=6: 1)=0.33$

${ }^{1}$ H NMR $\left(400 \mathrm{MHz}, \mathrm{CDCl}_{3}\right): \delta=7.33-7.27(\mathrm{~m}, 2 \mathrm{H}), 7.23-7.16(\mathrm{~m}, 3 \mathrm{H}), 3.91(\mathrm{td}, J=8.2,5.0 \mathrm{~Hz}, 1 \mathrm{H}), 3.84(\mathrm{dd}$, $J=8.4,6.9 \mathrm{~Hz}, 1 \mathrm{H}), 3.76(\mathrm{dt}, J=8.4,7.4 \mathrm{~Hz}, 1 \mathrm{H}), 3.47(\mathrm{dd}, J=8.4,6.6 \mathrm{~Hz}, 1 \mathrm{H}), 2.70(\mathrm{dd}, J=7.7,2.2 \mathrm{~Hz}, 2 \mathrm{H})$, 2.52 (hept, $J=7.1 \mathrm{~Hz}, 1 \mathrm{H}), 2.00$ (dtd, $J=12.5,7.6,5.0 \mathrm{~Hz}, 1 \mathrm{H}), 1.70-1.57$ (m, 1H) ppm.

${ }^{13}$ C NMR (101 MHz, $\mathrm{CDCl}_{3}$ ): $\delta=140.9,128.8$ (2C), 128.6 (2C), 126.2, 73.5, 68.0, 41.1, 39.5, 32.3 ppm.

All spectroscopic data corresponded to the reported literature values. ${ }^{[40]}$

\section{3-Isopropyl-1-tosylpyrrolidine (4v)}

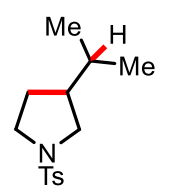

$4 \mathbf{v}$

Synthesized according to GP F (Kessil lamp $427 \mathrm{~nm}$ ) from $N$-(2-bromoethyl)-4-methyl- $N$-(3-methylbut-2-en-1yl)benzenesulfonamide (2v) $\left(69.3 \mathrm{mg}, 200 \mu \mathrm{mol}, 1.00\right.$ equiv.). Purification by flash chromatography $\left(\mathrm{SiO}_{2}\right.$, $n$-pentane:EtOAc $=10: 1)$ afforded $\mathbf{4 v}(41.0 \mathrm{mg}, 153 \mu \mathrm{mol}, 77 \%)$ as a colourless oil.

Analytical data of $4 v$ :

TLC $\left(\mathrm{SiO}_{2}\right): \mathrm{R}_{f}(n$-pentane:EtOAc $=10: 1)=0.31$ 
${ }^{1} \mathbf{H}$ NMR (400 MHz, $\left.\mathrm{CDCl}_{3}\right): \delta=7.70(\mathrm{~d}, J=8.2 \mathrm{~Hz}, 2 \mathrm{H}), 7.31(\mathrm{~d}, J=8.1 \mathrm{~Hz}, 2 \mathrm{H}), 3.44(\mathrm{dd}, J=9.7,7.7 \mathrm{~Hz}, 1 \mathrm{H})$, $3.36(\mathrm{ddd}, J=9.7,8.5,2.3 \mathrm{~Hz}, 1 \mathrm{H}), 3.14(\mathrm{td}, J=9.9,6.8 \mathrm{~Hz}, 1 \mathrm{H}), 2.77(\mathrm{t}, J=9.6 \mathrm{~Hz}, 1 \mathrm{H}), 2.42(\mathrm{~s}, 3 \mathrm{H}), 1.95-1.85$ $(\mathrm{m}, 1 \mathrm{H}), 1.74-1.62(\mathrm{~m}, 1 \mathrm{H}), 1.41-1.29(\mathrm{~m}, 2 \mathrm{H}), 0.84(\mathrm{~d}, J=1.1 \mathrm{~Hz}, 3 \mathrm{H}), 0.82(\mathrm{~d}, J=1.1 \mathrm{~Hz}, 3 \mathrm{H}) \mathrm{ppm}$.

${ }^{13} \mathrm{C}$ NMR (101 MHz, $\left.\mathrm{CDCl}_{3}\right): \delta=143.4,134.0,129.7$ (2C), 127.6 (2C), 52.2, 48.2, 46.5, 31.9, 30.1, 21.6, 21.4, $21.1 \mathrm{ppm}$.

All spectroscopic data corresponded to the reported literature values. ${ }^{[0]}$

\section{3-Methyl-1-tosylpyrrolidine (4w)}

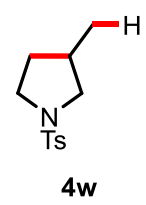

Synthesized according to GPF (Kessil lamp $427 \quad \mathrm{~nm}$ ) from $N$-allyl- $N$-(2-bromoethyl)4-methylbenzenesulfonamide (2w) $\left(63.7 \mathrm{mg}, 200 \mu \mathrm{mol}, 1.00\right.$ equiv.). Purification by flash chromatography $\left(\mathrm{SiO}_{2}\right.$, $n$-pentane:EtOAc $=6: 1)$ afforded $\mathbf{4 w}(26.0 \mathrm{mg}, 109 \mu \mathrm{mol}, 54 \%)$ as a white solid.

Analytical data of $\mathbf{4 w}$ :

TLC $\left(\mathrm{SiO}_{2}\right): \mathrm{R}_{f}\left(n\right.$-pentane: $\left.\mathrm{Et}_{2} \mathrm{O}=6: 1\right)=0.33$

${ }^{1} \mathbf{H}$ NMR $\left(400 \mathrm{MHz}, \mathrm{CDCl}_{3}\right): \delta=7.71(\mathrm{~d}, J=8.3 \mathrm{~Hz}, 2 \mathrm{H}), 7.32(\mathrm{~d}, J=8.1 \mathrm{~Hz}, 2 \mathrm{H}), 3.42(\mathrm{dd}, J=9.7,7.1 \mathrm{~Hz}, 1 \mathrm{H})$, 3.34 (ddd, $J=9.9,8.2,4.1 \mathrm{~Hz}, 1 \mathrm{H}), 3.22$ (ddd, $J=9.8,8.2,7.2 \mathrm{~Hz}, 1 \mathrm{H}), 2.75$ (dd, $J=9.7,7.8 \mathrm{~Hz}, 1 \mathrm{H}$ ), 2.43 (s, $3 \mathrm{H}), 2.18-2.05(\mathrm{~m}, 1 \mathrm{H}), 1.96-1.84(\mathrm{~m}, 1 \mathrm{H}), 1.35(\mathrm{dq}, J=12.3,8.4 \mathrm{~Hz}, 1 \mathrm{H}), 0.92(\mathrm{~d}, J=6.7 \mathrm{~Hz}, 3 \mathrm{H}) \mathrm{ppm}$.

${ }^{13}$ C NMR (101 MHz, $\mathrm{CDCl}_{3}$ ): $\delta=143.4,134.2,129.7$ (2C), 127.7 (2C), 54.9, 47.8, 33.5, 33.4, 21.7, 17.8 ppm. All spectroscopic data corresponded to the reported literature values. ${ }^{[41]}$

\section{2-Tosyl-2-azaspiro[4.5]decane (4x)}

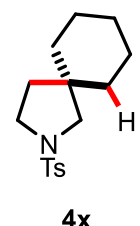

Synthesized according to GP F (Kessil lamp $427 \mathrm{~nm}$ ) from $N$-(cyclohex-1-en-1-ylmethyl)- $N$-(2-hydroxyethyl)-4methylbenzenesulfonamide (2x) $\left(74.5 \mathrm{mg}, 200 \mu \mathrm{mol}, 1.00\right.$ equiv.). Purification by flash chromatography $\left(\mathrm{SiO}_{2}\right.$, $n$-pentane:EtOAc $=10: 1)$ afforded $\mathbf{4 x}(30.0 \mathrm{mg}, 102 \mu \mathrm{mol}, 51 \%)$ as a colourless oil.

Analytical data of $\mathbf{4 x}$ :

TLC $\left(\mathrm{SiO}_{2}\right): \mathrm{R}_{f}(n$-pentane:EtOAc $=10: 1)=0.28$

${ }^{1} \mathbf{H}$ NMR $\left(400 \mathrm{MHz}, \mathrm{CDCl}_{3}\right): \delta=7.71(\mathrm{~d}, J=8.3 \mathrm{~Hz}, 2 \mathrm{H}), 7.31(\mathrm{~d}, J=7.7 \mathrm{~Hz}, 2 \mathrm{H}), 3.25(\mathrm{t}, J=7.1 \mathrm{~Hz}, 2 \mathrm{H}), 3.04$ (s, 2H), $2.42(\mathrm{~s}, 3 \mathrm{H}), 1.64-1.51(\mathrm{~m}, 2 \mathrm{H}), 1.43-1.17(\mathrm{~m}, 10 \mathrm{H}) \mathrm{ppm}$.

${ }^{13} \mathrm{C}$ NMR (101 MHz, $\left.\mathrm{CDCl}_{3}\right): \delta=143.3,134.0,129.7$ (2C), 127.6 (2C), 57.9, 46.5, 42.6, 37.1, 35.5, 26.0, 23.4, $21.7 \mathrm{ppm}$.

IR (ATR): 2922 (m), 2853 (w), 1450 (m), 1342 (s), 1158 (s), 1095 (s), 816 (w), 662 (s), 588 (s), 545 (s) cm . 
HRMS (ESI): calcd. for $\left[\mathrm{C}_{16} \mathrm{H}_{23} \mathrm{NO}_{2}+\mathrm{H}\right]^{+},[\mathrm{M}+\mathrm{H}]^{+}:$294.1528; found: 294.1506 .

\section{3-Isopropyl-4-methyl-1-tosylpyrrolidine (4y)}

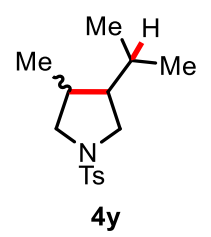

Synthesized according to GP F (Kessil lamp $427 \mathrm{~nm}$ ) from ( \pm )- $N$-(2-hydroxypropyl)-4-methyl- $N$-(3-methylbut-2en-1-yl)benzenesulfonamide (2y) $\left(72.1 \mathrm{mg}, 200 \mu \mathrm{mol}, 1.00\right.$ equiv.). Purification by flash chromatography $\left(\mathrm{SiO}_{2}\right.$, $n$-pentane:EtOAc $=10: 1)$ afforded $4 \mathbf{y}(51.0 \mathrm{mg}, 181 \mu \mathrm{mol}, 91 \%)$ as a colourless oil $(d . r .=65: 35)$.

Analytical data of $4 y$ :

TLC $\left(\mathrm{SiO}_{2}\right): \mathrm{R}_{f}(n$-pentane:EtOAc $=10: 1)=0.29$

${ }^{1} \mathbf{H}$ NMR (400 MHz, $\left.\mathrm{CDCl}_{3}\right)$ : major: $\delta=7.70(\mathrm{~d}, J=8.2 \mathrm{~Hz}, 2 \mathrm{H}), 7.30(\mathrm{~d}, J=8.3 \mathrm{~Hz}, 2 \mathrm{H}), 3.41(\mathrm{dd}, J=9.5,7.7$ $\mathrm{Hz}, 1 \mathrm{H}), 3.29$ (dd, $J=9.9,5.7 \mathrm{~Hz}, 1 \mathrm{H}), 3.15(\mathrm{~d}, J=9.7 \mathrm{~Hz}, 1 \mathrm{H}), 2.87(\mathrm{dd}, J=11.2,9.7 \mathrm{~Hz}, 1 \mathrm{H}), 2.42(\mathrm{~s}, 3 \mathrm{H}), 2.23$ $-2.11(\mathrm{~m}, 1 \mathrm{H}), 1.65-1.52(\mathrm{~m}, 1 \mathrm{H}), 1.49-1.38(\mathrm{~m}, 1 \mathrm{H}), 0.82(\mathrm{t}, J=6.7 \mathrm{~Hz}, 6 \mathrm{H}), 0.62(\mathrm{~d}, J=7.0 \mathrm{~Hz}, 3 \mathrm{H}) \mathrm{ppm}$. minor: $\delta=7.69(\mathrm{~d}, J=8.0 \mathrm{~Hz}, 2 \mathrm{H}), 7.31(\mathrm{~d}, J=9.2 \mathrm{~Hz}, 2 \mathrm{H}), 3.42(\mathrm{~d}, J=9.5 \mathrm{~Hz}, 1 \mathrm{H}), 3.36-3.27(\mathrm{~m}, 1 \mathrm{H}), 2.92$ (dd, $J=10.0,8.8 \mathrm{~Hz}, 1 \mathrm{H}), 2.72(\mathrm{dd}, J=9.6,8.3 \mathrm{~Hz}, 1 \mathrm{H}), 2.42(\mathrm{~s}, 3 \mathrm{H}), 1.93-1.80(\mathrm{~m}, 1 \mathrm{H}), 1.67-1.57(\mathrm{~m}, 1 \mathrm{H})$, $1.49-1.42(\mathrm{~m}, 1 \mathrm{H}), 0.91(\mathrm{~d}, J=6.6 \mathrm{~Hz}, 3 \mathrm{H}), 0.86(\mathrm{~d}, J=6.8 \mathrm{~Hz}, 3 \mathrm{H}), 0.76(\mathrm{~d}, J=6.7 \mathrm{~Hz}, 3 \mathrm{H}) \mathrm{ppm}$.

${ }^{13} \mathrm{C}$ NMR (101 MHz, $\left.\mathrm{CDCl}_{3}\right): \delta=143.4,143.3,134.5,133.6,129.7,127.7,127.4,56.1,55.5,52.0,50.3,50.1,49.8$, 36.0, 34.0, 28.8, 27.4, 21.9, 21.6, 21.5, 21.1, 18.5, 17.7, $13.0 \mathrm{ppm}$.

All spectroscopic data corresponded to the reported literature values. ${ }^{[42]}$

\section{3,3-Dichloro-4-isopropyl-1-tosylpyrrolidine (4z)}

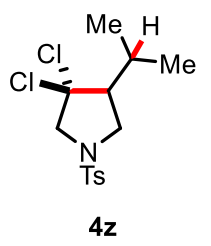

Synthesized according to GP F (Kessil lamp $427 \mathrm{~nm}$ ) from 4-methyl- $N$-(3-methylbut-2-en-1-yl)- $N$-(2,2,2trichloroethyl)benzenesulfonamide (3z) $(74.1 \mathrm{mg}, 200 \mu \mathrm{mol}, 1.00$ equiv.). Purification by flash chromatography $\left(\mathrm{SiO}_{2}, n\right.$-pentane:EtOAc $\left.=20: 1\right)$ afforded $\mathbf{4 z}(55.0 \mathrm{mg}, 163 \mu \mathrm{mol}, 82 \%)$ as a white solid.

Analytical data of 4z:

TLC $\left(\mathrm{SiO}_{2}\right): \mathrm{R}_{f}(n$-pentane:EtOAc $=20: 1)=0.19$

Mp: $135.5-136.2{ }^{\circ} \mathrm{C}$.

${ }^{1} \mathbf{H}$ NMR $\left(400 \mathrm{MHz}, \mathrm{CDCl}_{3}\right): \delta=7.72(\mathrm{~d}, J=8.3 \mathrm{~Hz}, 2 \mathrm{H}), 7.34(\mathrm{~d}, J=8.0 \mathrm{~Hz}, 2 \mathrm{H}), 4.11(\mathrm{~d}, J=11.9 \mathrm{~Hz}, 1 \mathrm{H}), 3.89$ (d, $J=11.8 \mathrm{~Hz}, 1 \mathrm{H}), 3.69(\mathrm{dd}, J=9.6,7.9 \mathrm{~Hz}, 1 \mathrm{H}), 3.05(\mathrm{dd}, J=10.5,9.6 \mathrm{~Hz}, 1 \mathrm{H}), 2.44(\mathrm{~s}, 3 \mathrm{H}), 2.30-2.15$ (m, $1 \mathrm{H}), 1.99-1.84(\mathrm{~m}, 1 \mathrm{H}), 1.15(\mathrm{~d}, J=6.6 \mathrm{~Hz}, 3 \mathrm{H}), 0.91(\mathrm{~d}, J=6.7 \mathrm{~Hz}, 3 \mathrm{H}) \mathrm{ppm}$.

${ }^{13} \mathrm{C}$ NMR (101 MHz, $\left.\mathrm{CDCl}_{3}\right): \delta=144.1,134.0,129.9$ (2C), 127.7 (2C), 87.8, 66.7, 59.1, 51.2, 29.6, 21.8 (2C), $21.2 \mathrm{ppm}$.

IR (ATR): 2966 (m), 1351 (m), 1167 (s), 1064 (m), 814 (w), 665 (s), 595 (m), 549 (m) cm¹. 
HRMS (ESI): calcd. for $\left[\mathrm{C}_{14} \mathrm{H}_{19} \mathrm{Cl}_{2} \mathrm{NO}_{2} \mathrm{~S}+\mathrm{H}\right]^{+},[\mathrm{M}+\mathrm{H}]^{+}$: 336.0586; found: 336.0560 .

\section{3,3-Dichloro-4-isopropyl-1-tosylpiperidine (4za)}

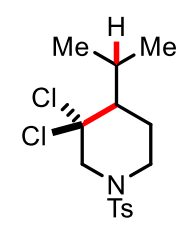

$4 z a$

Synthesized according to GPF (Kessil lamp $427 \mathrm{~nm}$ ) from 4-methyl- $N$-(4-methylpent-3-en-1-yl)- $N$-(2,2,2trichloroethyl)benzenesulfonamide (3za) $(77.0 \mathrm{mg}, 200 \mu \mathrm{mol}, 1.00$ equiv.). Purification by flash chromatography $\left(\mathrm{SiO}_{2}, n\right.$-pentane:EtOAc $\left.=10: 1\right)$ afforded $4 \mathbf{z a}(48.0 \mathrm{mg}, 137 \mu \mathrm{mol}, 69 \%)$ as a white solid.

Analytical data of $\mathbf{4 z a}$ :

TLC $\left(\mathrm{SiO}_{2}\right): \mathrm{R}_{f}(n$-pentane:EtOAc $=10: 1)=0.33$

Mp: $131.6-133.0^{\circ} \mathrm{C}$.

${ }^{1} \mathbf{H}$ NMR (400 MHz, CDCl $): \delta=7.67(\mathrm{~d}, J=8.3 \mathrm{~Hz}, 2 \mathrm{H}), 7.33(\mathrm{~d}, J=8.0 \mathrm{~Hz}, 2 \mathrm{H}), 4.27(\mathrm{dd}, J=12.5,2.2 \mathrm{~Hz}, 1 \mathrm{H})$, $3.89(\mathrm{ddt}, J=11.8,4.6,2.3 \mathrm{~Hz}, 1 \mathrm{H}), 2.82(\mathrm{~d}, J=12.5 \mathrm{~Hz}, 1 \mathrm{H}), 2.44(\mathrm{~s}, 3 \mathrm{H}), 2.46-2.36(\mathrm{~m}, 1 \mathrm{H}), 2.34(\mathrm{td}, J=12.1$, $2.8 \mathrm{~Hz}, 1 \mathrm{H}), 1.90-1.74(\mathrm{~m}, 1 \mathrm{H}), 1.68-1.56(\mathrm{~m}, 2 \mathrm{H}), 0.98(\mathrm{~d}, J=4.5 \mathrm{~Hz}, 3 \mathrm{H}), 0.96(\mathrm{~d}, J=4.7 \mathrm{~Hz}, 3 \mathrm{H}) \mathrm{ppm}$.

${ }^{13}$ C NMR (101 MHz, $\left.\mathrm{CDCl}_{3}\right): \delta=144.1,134.0,130.0,127.7,90.9,62.0,55.5,46.3,27.7,24.2,24.0,21.7,18.3$ ppm. IR (ATR): 2961 (w), 1391 (m), 1262 (w), 1162 (s), 1091 (m), 946 (m), 815 (m), 743 (s), 654 (s), 548 (s) cm ${ }^{-1}$.

HRMS (ESI): calcd. for $\left[\mathrm{C}_{15} \mathrm{H}_{21} \mathrm{Cl}_{2} \mathrm{NO}_{2} \mathrm{~S}+\mathrm{H}\right]^{+},[\mathrm{M}+\mathrm{H}]^{+}: 350.0743$; found: 350.0749 .

\subsection{Non-working Substrates}

Following compounds were used in GP F. However, no desired intramolecular cyclization reaction was observed.

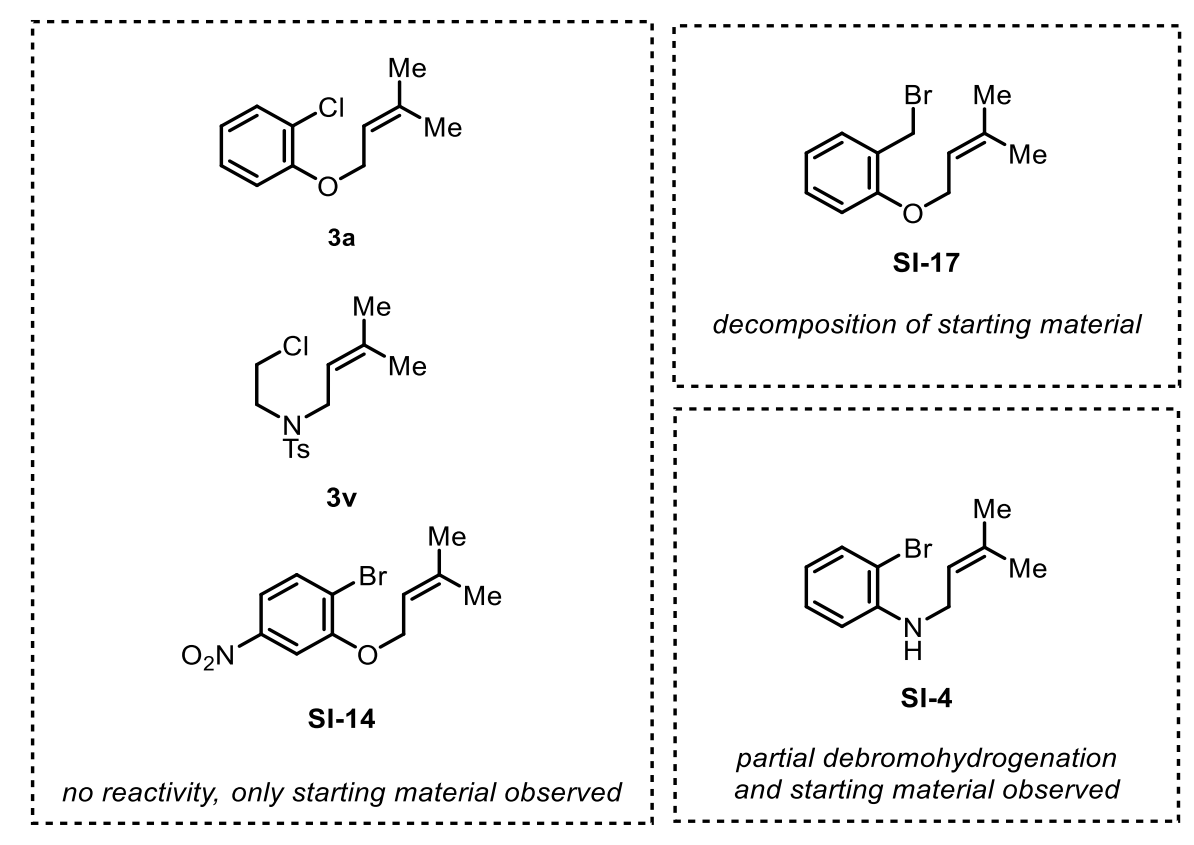




\section{References}

[1] J. A. Balzani V., Ceroni P., Photochemistry and Photophysics, Wiley-VCH, 2014. pp. 103-123.

[2] N. A. Romero, D. A. Nicewicz, Chem. Rev. 2016, 116, 10075-10166.

[3] Y.-C. Chang, Y.-C. Lee, M.-F. Chang, F.-E. Hong, J. Organomet. Chem. 2016, 808, 23-33.

[4] D. Gudat, A. Haghverdi, H. Hupfer, M. Nieger, Chem. A Eur. J. 2000, 6, 3414-3425.

[5] T. Lundrigan, C. H. Tien, K. N. Robertson, A. W. H. Speed, Chem. Commun. 2020, 56, 8027-8030.

[6] B. S. N. Huchenski, K. N. Robertson, A. W. H. Speed, Eur. J. Org. Chem. 2020, 2020, 5140-5144.

[7] J. H. Reed, P. A. Donets, S. Miaskiewicz, N. Cramer, Angew. Chem. Int. Ed. 2019, 58, 8893-8897.

[8] M. R. Adams, C. H. Tien, B. S. N. Huchenski, M. J. Ferguson, A. W. H. Speed, Angew. Chem. Int. Ed. 2017, 56, 6268-6271.

[9] A. E. Finholt, A. C. Bond, H. I. Schlesinger, J. Am. Chem. Soc. 1947, 69, 1199-1203.

[10] S. Burck, D. Gudat, K. Nättinen, M. Nieger, M. Niemeyer, D. Schmid, Eur. J. Inorg. Chem. 2007, 2007, 5112-5119.

[11] H. X. Feng, Y. Y. Wang, J. Chen, L. Zhou, Adv. Synth. Catal. 2015, 357, 940-944.

[12] M. Koy, P. Bellotti, F. Katzenburg, C. G. Daniliuc, F. Glorius, Angew. Chem. Int. Ed. 2020, 59, 2375-2379.

[13] W. Russell Bowman, S. L. Krintel, M. B. Schilling, Org. Biomol. Chem. 2004, 2, 585.

[14] Y. Gao, W. Xiong, H. Chen, W. Wu, J. Peng, Y. Gao, H. Jiang, J. Org. Chem. 2015, 80, 7456-7467.

[15] P. S. M. Amado, L. M. T. Frija, J. A. S. Coelho, P. M. O’Neill, M. L. S. Cristiano, J. Org. Chem. 2021, 86, 10608-10620.

[16] J. Ban, M. Lim, S. Shabbir, J. Baek, H. Rhee, Synthesis (Stuttg). 2020, 52, 917-927.

[17] A. Duncan, R. Widenhoefer, Synlett 2010, 2010, 419-422.

[18] C. Ollivier, P. Renaud, J. Am. Chem. Soc. 2001, 123, 4717-4727.

[19] K. Takami, S. Mikami, H. Yorimitsu, H. Shinokubo, K. Oshima, Tetrahedron 2003, 59, 6627-6635.

[20] A. I. Meyers, E. W. Collington, Tetrahedron 1971, 27, 5979-5985.

[21] H. Lei, J. Atkinson, J. Org. Chem. 2000, 65, 2560-2567.

[22] A. A. Mikhaylov, A. D. Dilman, R. A. Novikov, Y. A. Khoroshutina, M. I. Struchkova, D. E. Arkhipov, Y. V. Nelyubina, A. A. Tabolin, S. L. Ioffe, Tetrahedron Lett. 2016, 57, 11-14.

[23] O. Tamura, T. Mitsuya, X. Huang, Y. Tsutsumi, S. Hattori, H. Ishibashi, J. Org. Chem. 2005, 70, 1072010725.

[24] H. Kim, C. Lee, Org. Lett. 2002, 4, 4369-4371.

[25] K. Wen, Z. Wu, B. Chen, J. Chen, W. Zhang, Org. Biomol. Chem. 2018, 16, 5618-5625.

[26] A. R. O. Venning, M. R. Kwiatkowski, J. E. Roque Peña, B. C. Lainhart, A. A. Guruparan, E. J. Alexanian, J. Am. Chem. Soc. 2017, 139, 11595-11600.

[27] X. Dong, R. Sang, Q. Wang, X.-Y. Tang, M. Shi, Chem. Eur. J. 2013, 19, 16910-16915.

[28] A. Roedig, K. Grohe, G. Märkl, Chem. Ber. 1966, 99, 121-129.

[29] K. Watanabe, T. Mino, C. Hatta, E. Ishikawa, Y. Yoshida, M. Sakamoto, Eur. J. Org. Chem. 2017, 2017, 3612-3619.

[30] N. Hayashi, I. Shibata, A. Baba, Org. Lett. 2004, 6, 4981-4983. 
[31] C. Bolm, M. Ewald, M. Zehnder, M. A. Neuburger, Chem. Ber. 1992, 125, 453-458.

[32] H. Yorimitsu, H. Shinokubo, K. Oshima, Bull. Chem. Soc. Jpn. 2001, 74, 225-235.

[33] T. Ohmura, S. Kusaka, T. Torigoe, M. Suginome, Adv. Synth. Catal. 2019, 361, 4448-4453.

[34] C. A. D. Caiuby, A. Ali, V. T. Santana, F. W. S. De Lucas, M. S. Santos, A. G. Corrêa, O. R. Nascimento, H. Jiang, M. W. Paixão, RSC Adv. 2018, 8, 12879-12886.

[35] K. Fujita, T. Nakamura, H. Yorimitsu, K. Oshima, J. Am. Chem. Soc. 2001, 123, 3137-3138.

[36] A. Ekomié, G. Lefèvre, L. Fensterbank, E. Lacôte, M. Malacria, C. Ollivier, A. Jutand, Angew. Chem. 2012, $124,7048-7052$.

[37] A. Inoue, H. Shinokubo, K. Oshima, Org. Lett. 2000, 2, 651-653.

[38] J. Y. Hwang, J. H. Baek, T. Il Shin, J. H. Shin, J. W. Oh, K. P. Kim, Y. You, E. J. Kang, Org. Lett. 2016, $18,4900-4903$.

[39] D. Zhu, L. Lv, Z. Qiu, C.-J. Li, J. Org. Chem. 2019, 84, 6312-6322.

[40] J. G. Kim, Y. H. Son, J. W. Seo, E. J. Kang, Eur. J. Org. Chem. 2015, 2015, 1781-1789.

[41] S. Smoleń, A. Wincenciuk, O. Drapała, D. Gryko, Synthesis (Stuttg). 2021, 53, 1645-1653.

[42] T.-Y. Lin, C.-Z. Zhu, P. Zhang, Y. Wang, H.-H. Wu, J.-J. Feng, J. Zhang, Angew. Chem. Int. Ed. 2016, 55, 10844-10848. 


\section{NMR Spectra}

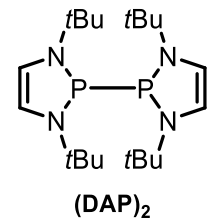

${ }^{1} \mathrm{H} N M R, 400 \mathrm{MHz}, \mathrm{CD}_{3} \mathrm{CN}$<smiles>CC(C)(C)CN1C=CN(C(C)(C)C)P1P1N(CC(C)(C)C)C=CN1C(C)(C)C</smiles>

$(\text { DAP })_{2}$

${ }^{31} \mathrm{P}$ NMR, $162 \mathrm{MHz}, \mathrm{CD}_{3} \mathrm{CN}$

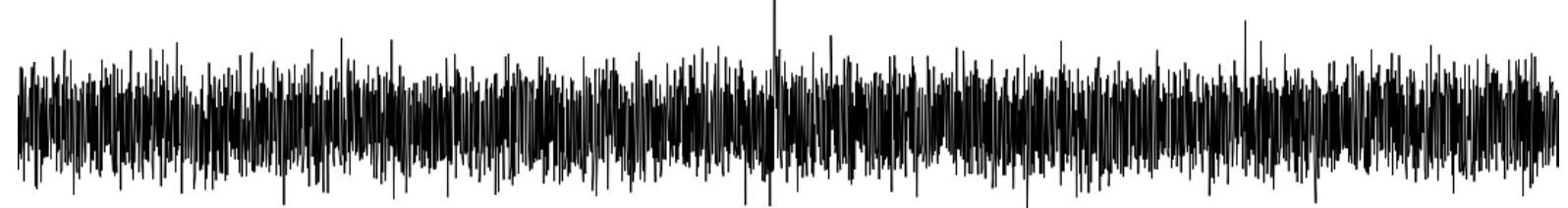

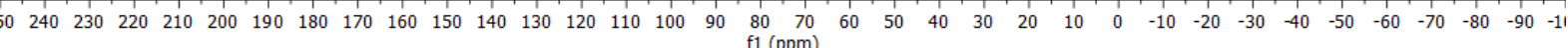




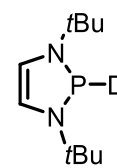

DAP-D

${ }^{1} \mathrm{H}$ NMR, $400 \mathrm{MHz}, \mathrm{C}_{6} \mathrm{D}_{6}$

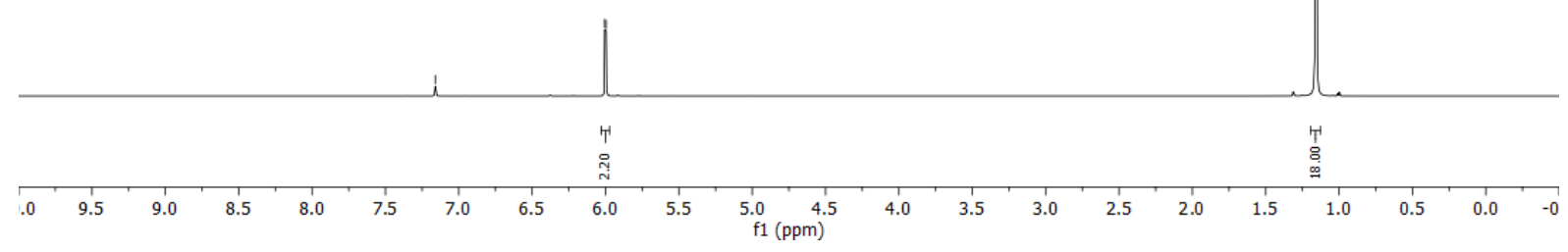

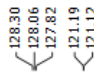
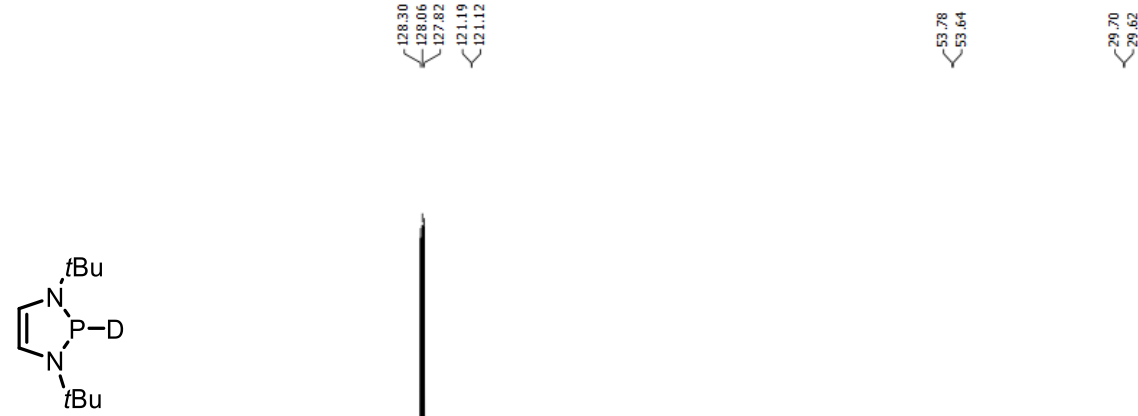

DAP-D

${ }^{13} \mathrm{C}$ NMR, $101 \mathrm{MHz}, \mathrm{C}_{6} \mathrm{D}_{6}$

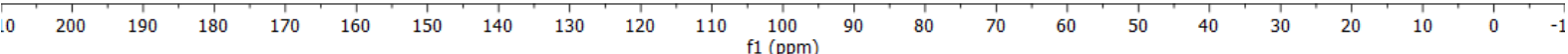




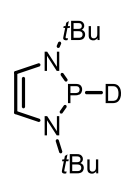

DAP-D

${ }^{31} \mathrm{P} N M R, 162 \mathrm{MHz}, \mathrm{C}_{6} \mathrm{D}_{6}$

\begin{tabular}{llllllllllllllllllllllllllllllllllllllllll}
\hline 0 & 240 & 230 & 220 & 210 & 200 & 190 & 180 & 170 & 160 & 150 & 140 & 130 & 120 & 110 & 100 & 90 & 80 & 70 & 60 & 50 & 40 & 30 & 20 & 10 & 0 & -10 & -20 & -30 & -40 & -50 & -60 & -70 & -80 & -90
\end{tabular}

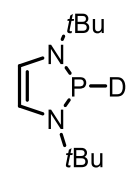

DAP-D

${ }^{1} \mathrm{H}$ NMR, $400 \mathrm{MHz}, \mathrm{CD}_{3} \mathrm{CN}$

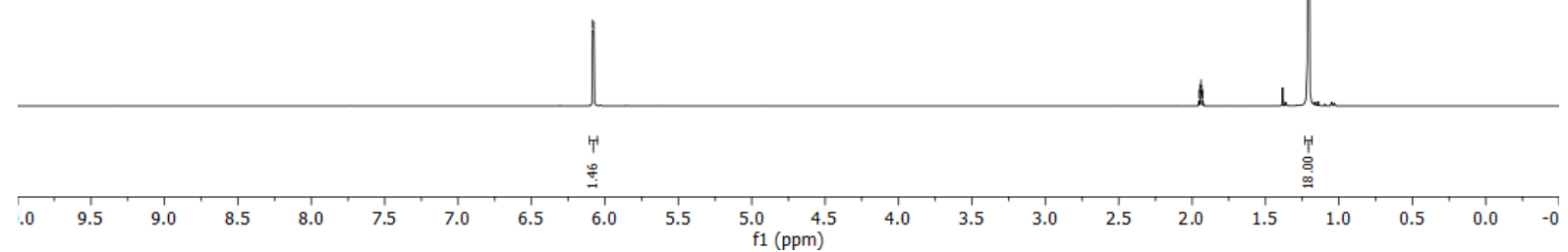



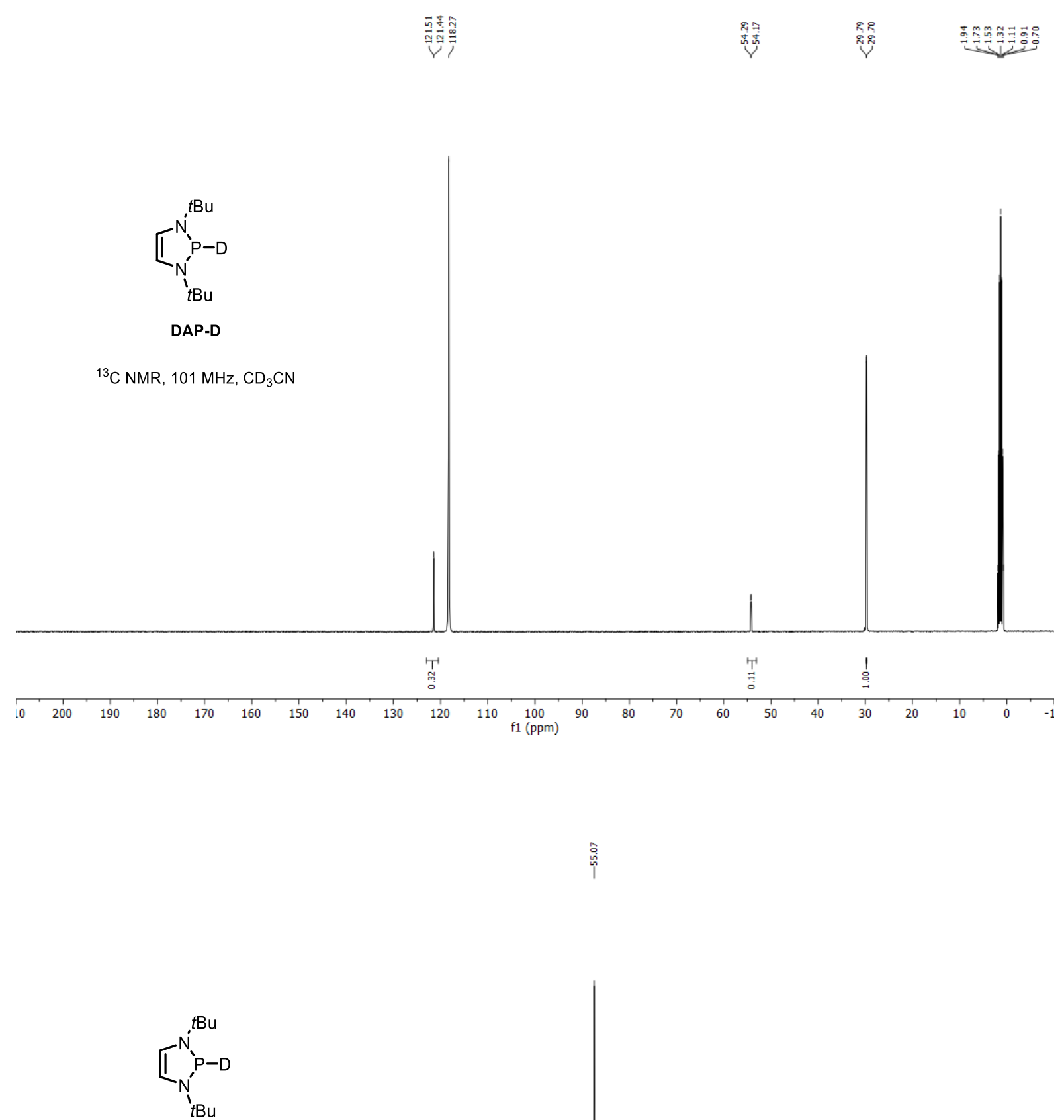

DAP-D

${ }^{31} \mathrm{P}$ NMR, $162 \mathrm{MHz}, \mathrm{CD}_{3} \mathrm{CN}$ 
<smiles>CC(=O)N1C=CN(C)C1I</smiles>

DAP-I

${ }^{1} \mathrm{H} \mathrm{NMR}, 400 \mathrm{MHz}, \mathrm{CD}_{3} \mathrm{CN}$

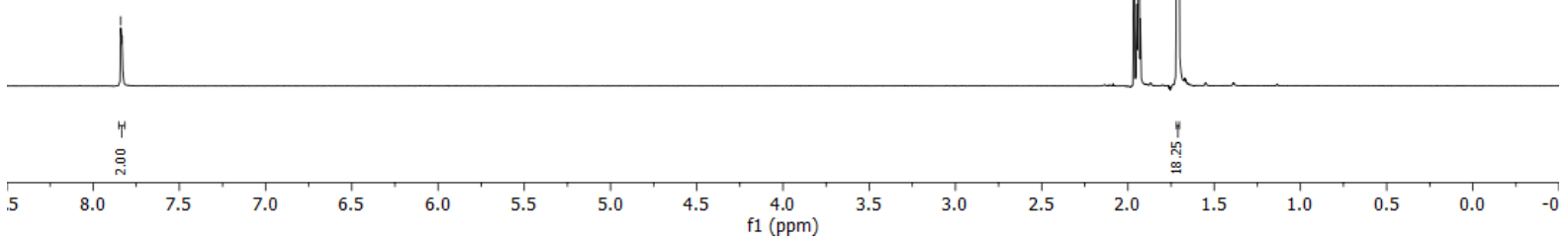

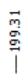

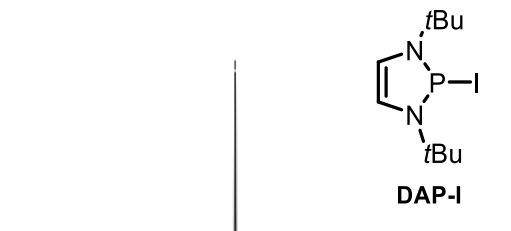

${ }^{31} \mathrm{P}$ NMR, $162 \mathrm{MHz}, \mathrm{CD}_{3} \mathrm{CN}$

$\begin{array}{lllllllllllllllllllllllllllllllllllll}240 & 230 & 220 & 210 & 200 & 190 & 180 & 170 & 160 & 150 & 140 & 130 & 120 & 110 & 100 & 90 & 80 & 70 & 60 & 50 & 40 & 30 & 20 & 10 & 0 & -10 & -20 & -30 & -40 & -50 & -60 & -70 & -80 & -90 & -1\end{array}$ 


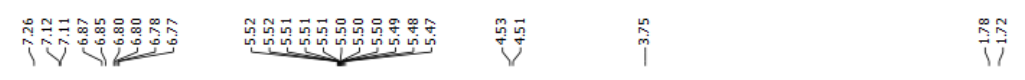

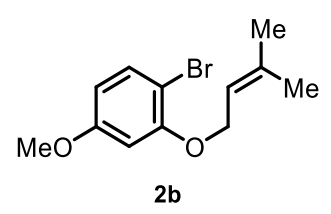

${ }^{1} \mathrm{H} \mathrm{NMR}, 400 \mathrm{MHz}, \mathrm{CDCl}_{3}$
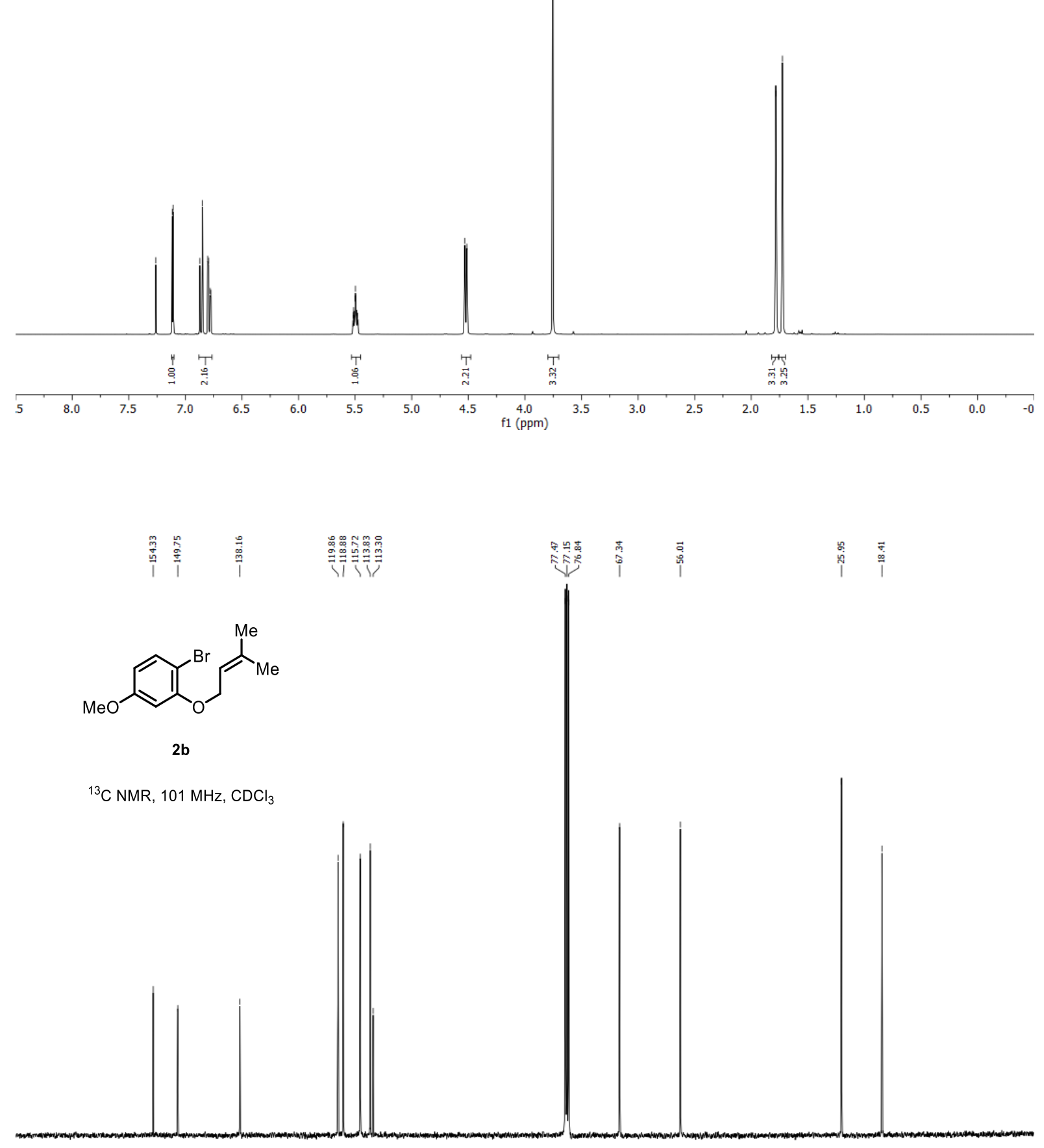

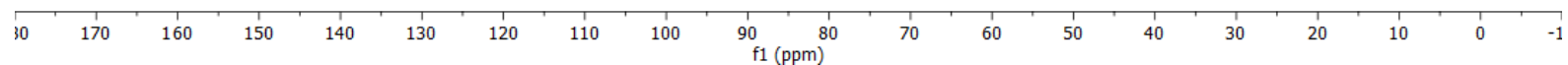




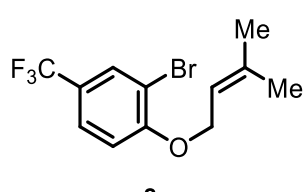

2c

${ }^{1} \mathrm{H} \mathrm{NMR}, 400 \mathrm{MHz}, \mathrm{CDCl}_{3}$
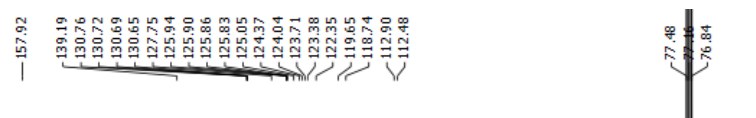

।

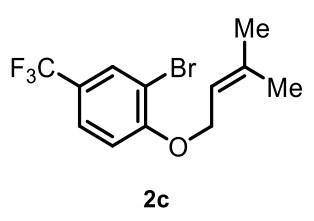

${ }^{13} \mathrm{C} \mathrm{NMR}, 101 \mathrm{MHz}, \mathrm{CDCl}_{3}$

\section{唯}

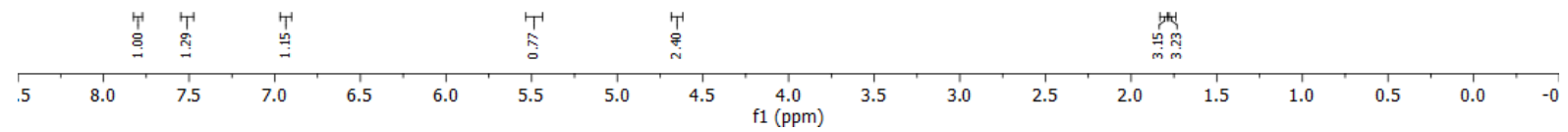
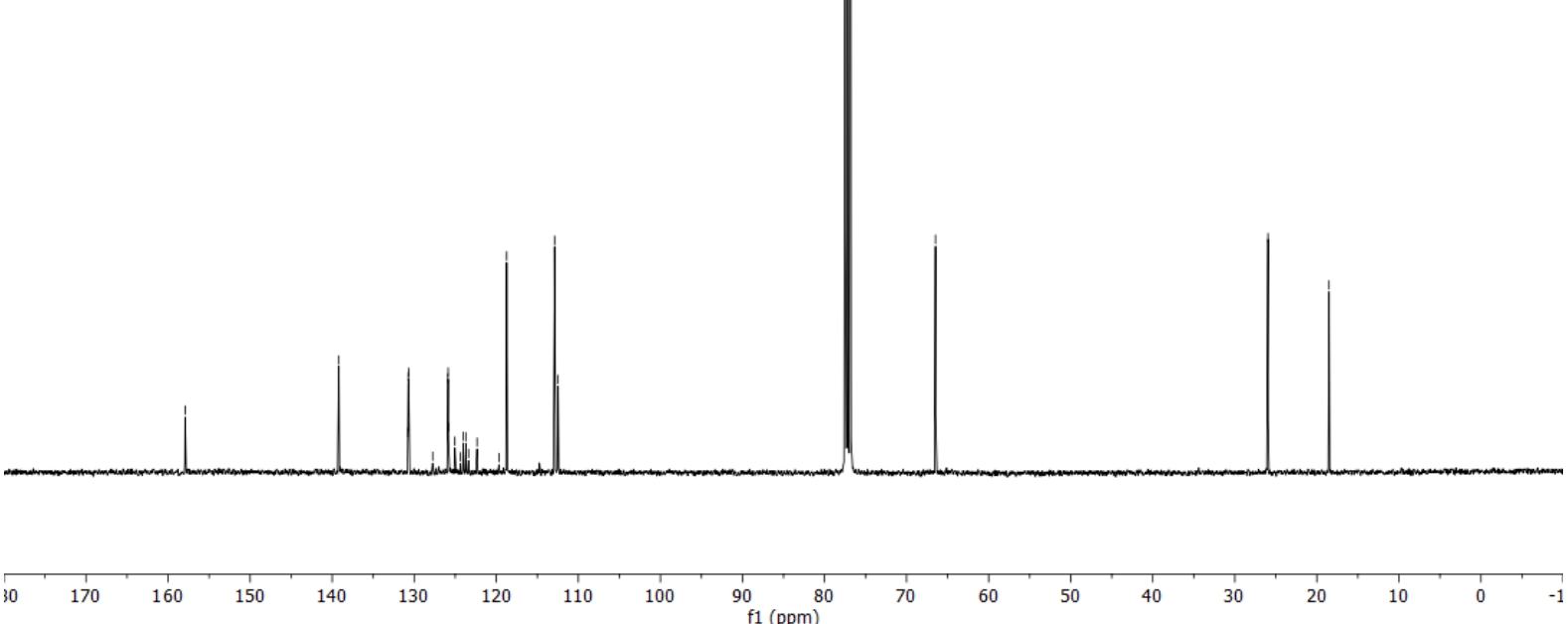


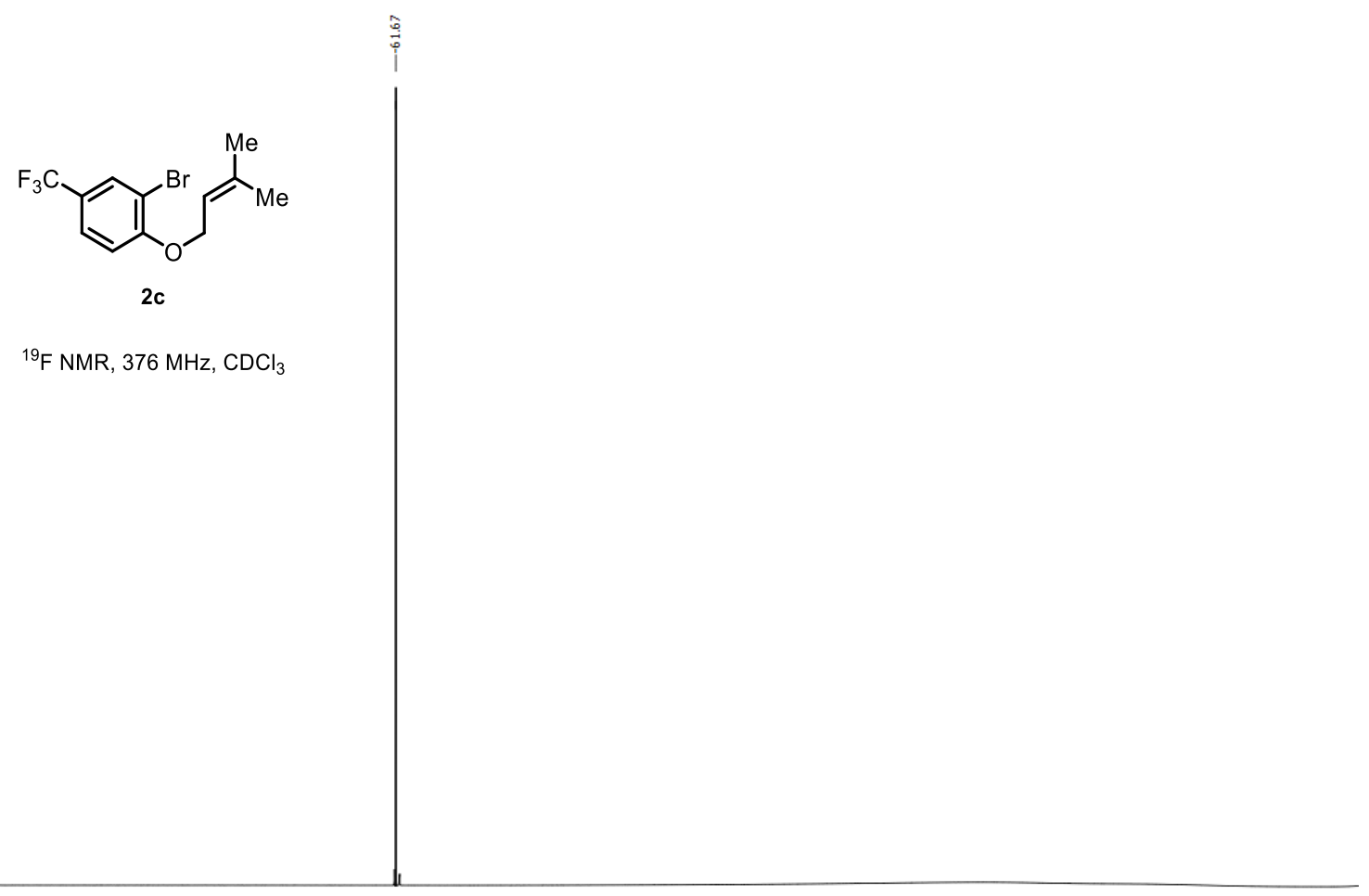

\begin{tabular}{rlllllllllllllllllllllll}
\hline 10 & 0 & -10 & -20 & -30 & -40 & -50 & -60 & -70 & -80 & -90 & -100 & -110 & -120 & -130 & -140 & -150 & -160 & -170 & -180 & -190 & -200 & -210 & 1
\end{tabular} 


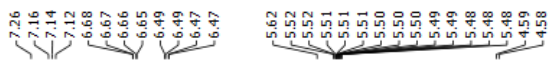

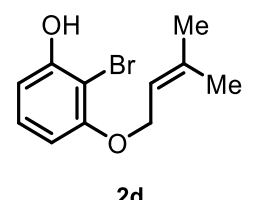

${ }^{1} \mathrm{H}$ NMR, $400 \mathrm{MHz}, \mathrm{CDCl}_{3}$

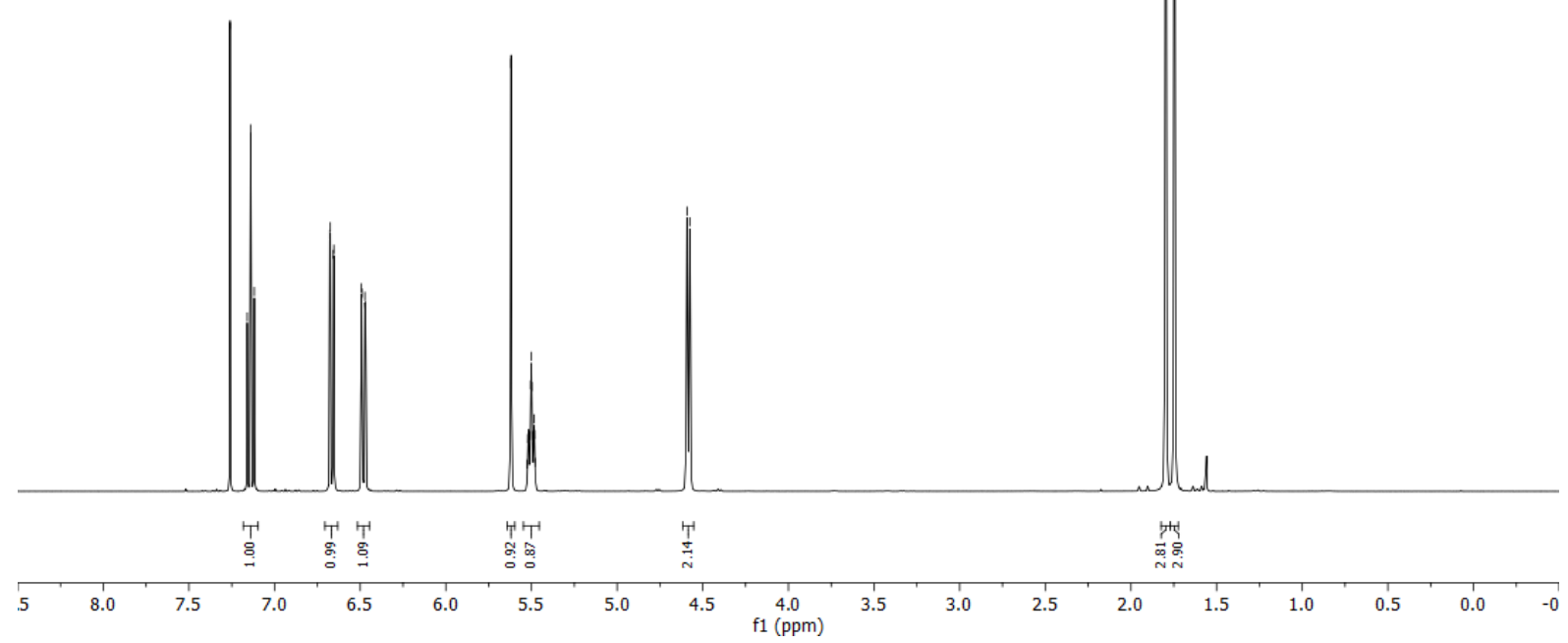

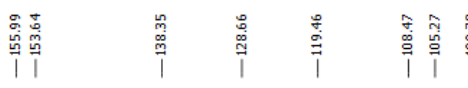

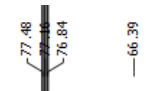

$\stackrel{\substack{0 \\ i}}{i}$<smiles>CC(C)=CCOc1cccc(O)c1Br</smiles>

${ }^{13} \mathrm{C} \mathrm{NMR}, 101 \mathrm{MHz}, \mathrm{CDCl}_{3}$

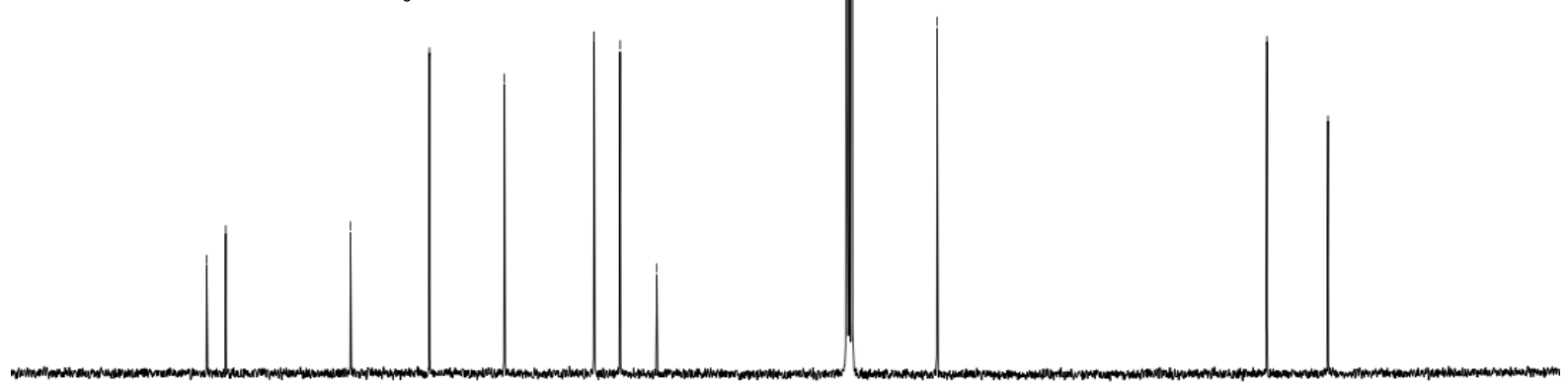

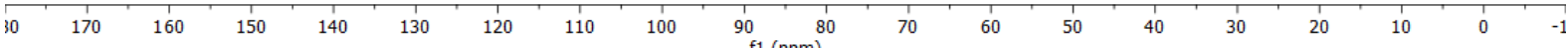




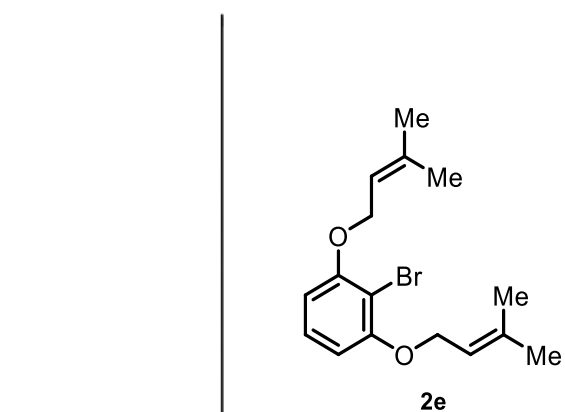

${ }^{1} \mathrm{H} \mathrm{NMR}, 400 \mathrm{MHz}, \mathrm{CDCl}_{3}$
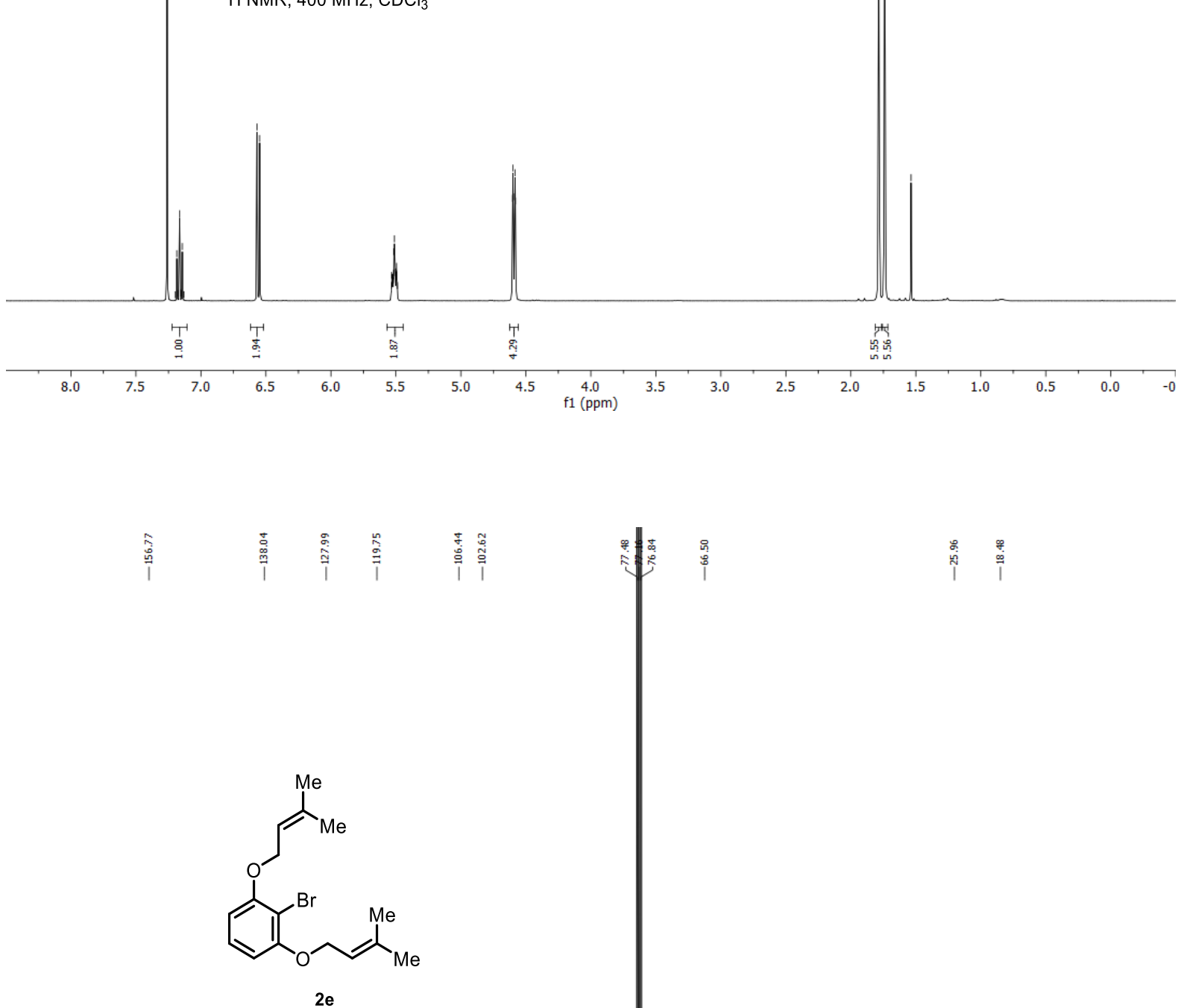

${ }^{13} \mathrm{C} \mathrm{NMR}, 101 \mathrm{MHz}, \mathrm{CDCl}_{3}$

$\begin{array}{llllllllll}10 & 170 & 160 & 150 & 140 & 130 & 120 & 110 & 100 & 90 \\ & & & & & & & 1 \\ \mathrm{f} 1(\mathrm{ppm})\end{array}$

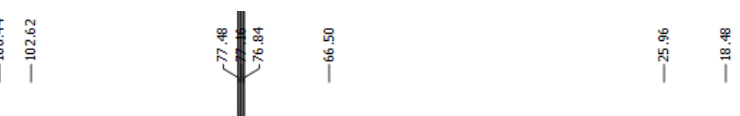

$\mid$

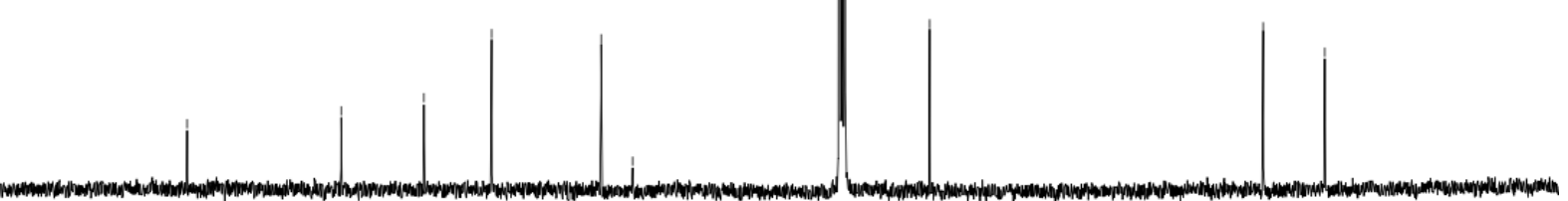



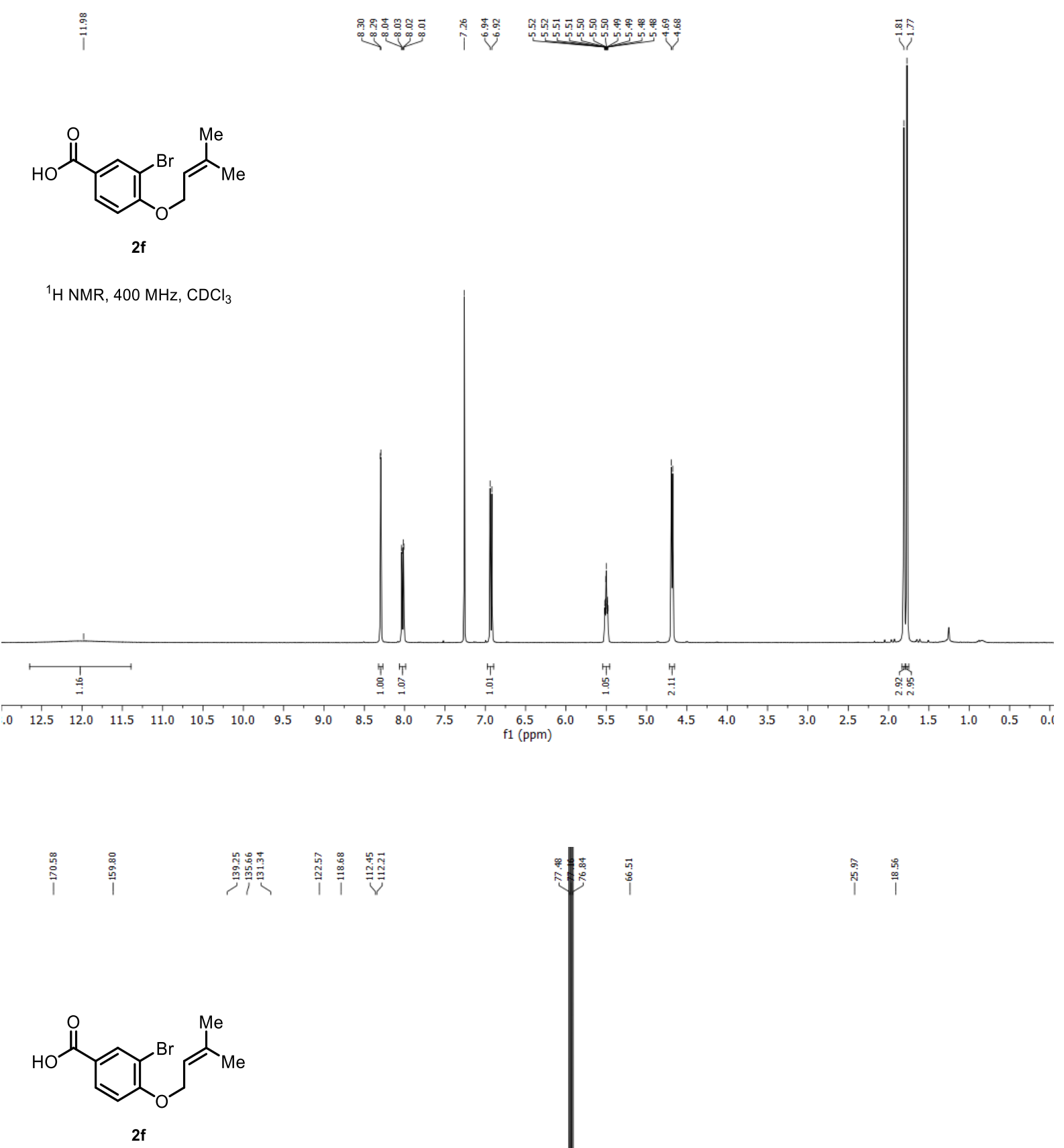

${ }^{13} \mathrm{C} \mathrm{NMR,}, 101 \mathrm{MHz}, \mathrm{CDCl}_{3}$

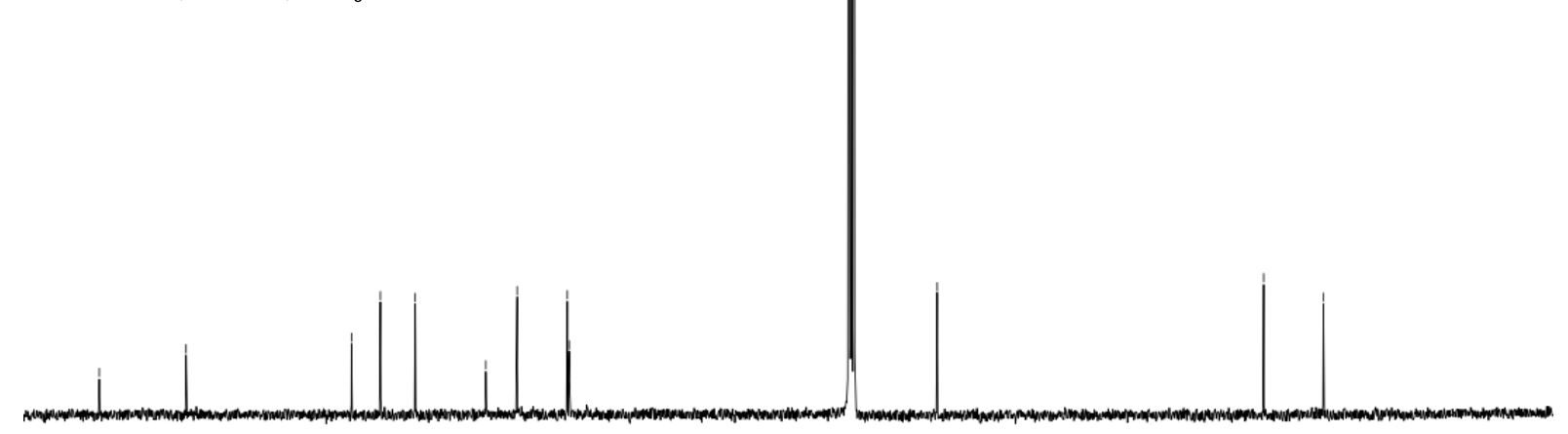

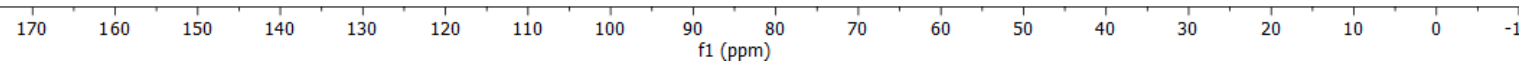


<smiles>CC(C)=[N+]([O-])CCOc1c(Cl)cccc1Br</smiles>

$2 \mathrm{~h}$

${ }^{1} \mathrm{H} \mathrm{NMR}, 400 \mathrm{MHz}, \mathrm{CDCl}_{3}$

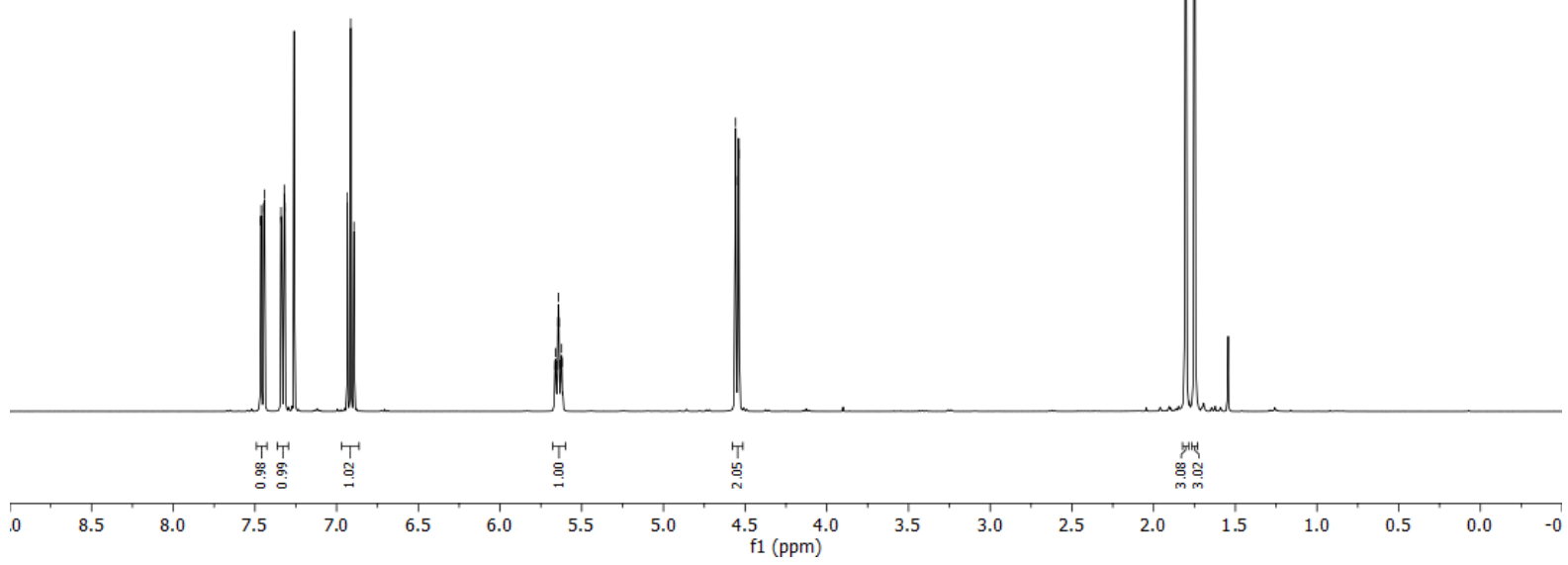

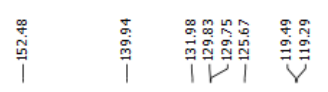<smiles>O=C(CCOc1c(Cl)cccc1Br)[N+](=O)[O-]</smiles>

$2 \mathrm{~h}$

${ }^{13} \mathrm{C} \mathrm{NMR}, 101 \mathrm{MHz}, \mathrm{CDCl}_{3}$

$\begin{array}{llllllllll}10 & 170 & 160 & 150 & 140 & 130 & 120 & 110 & 100 & 90 \\ \mathrm{f} 1(\mathrm{ppm}) & 80\end{array}$

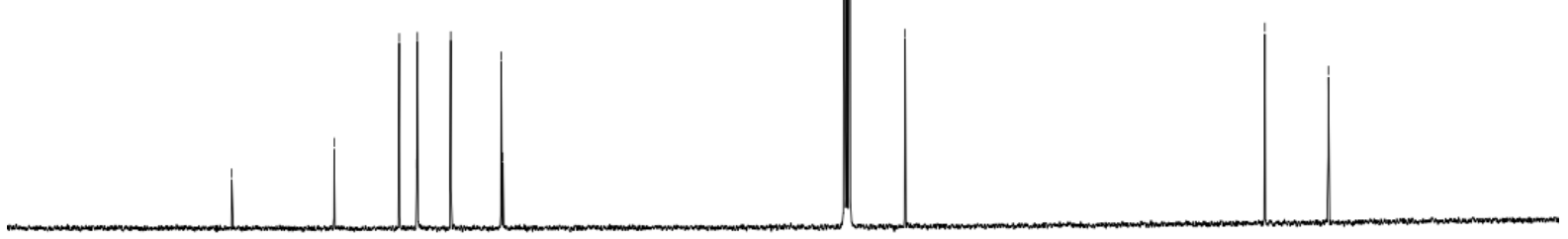




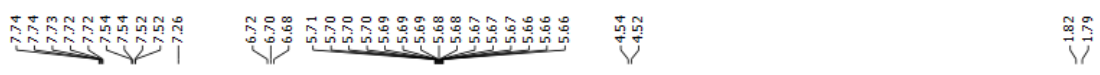

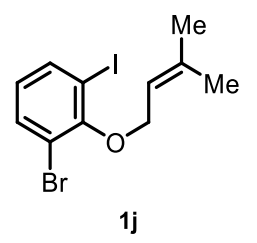

${ }^{1} \mathrm{H} \mathrm{NMR}, 400 \mathrm{MHz}, \mathrm{CDCl}_{3}$

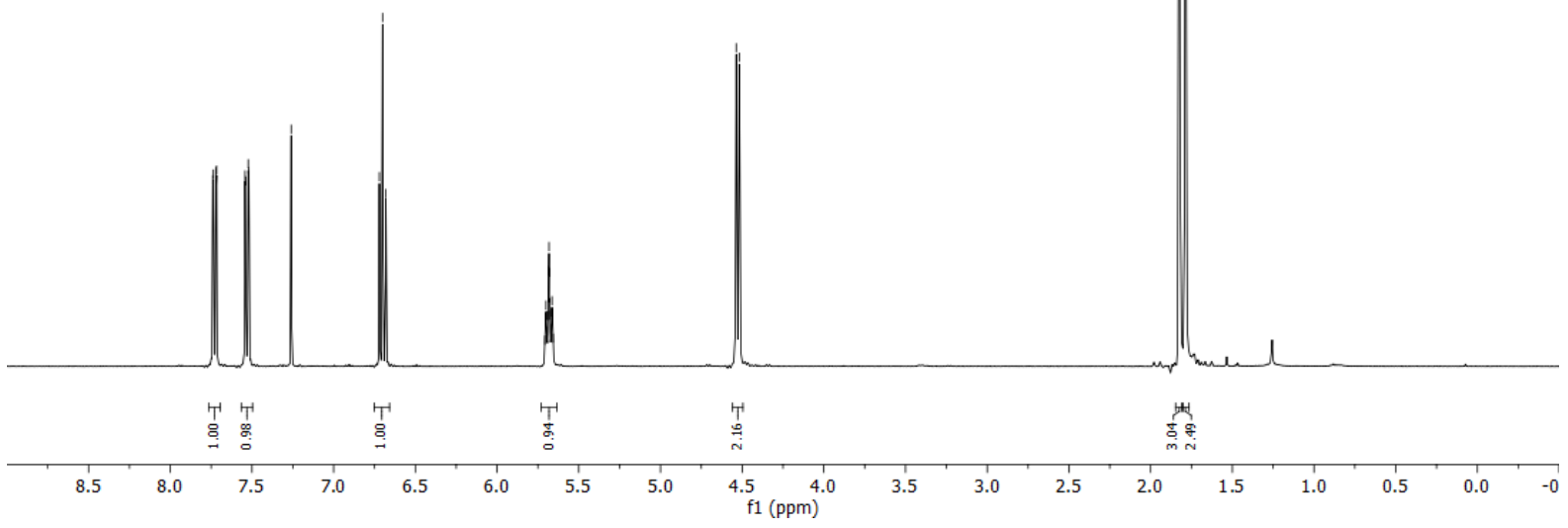

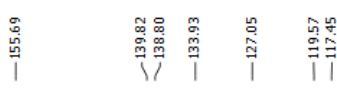<smiles>CC(C)=CCOc1c(Br)cccc1I</smiles>

1j

${ }^{13} \mathrm{C} \mathrm{NMR}, 101 \mathrm{MHz}, \mathrm{CDCl}_{3}$

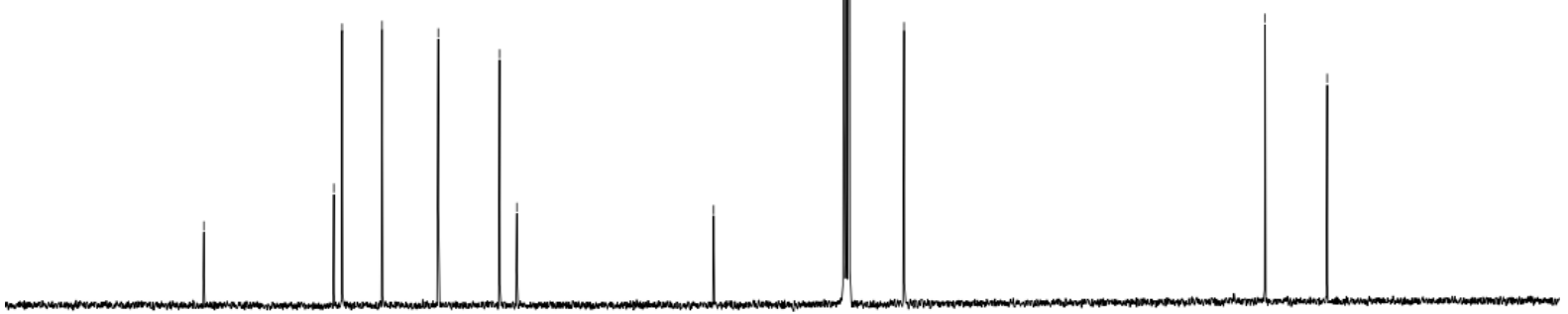

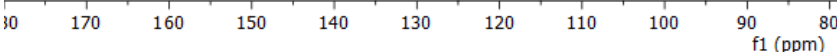




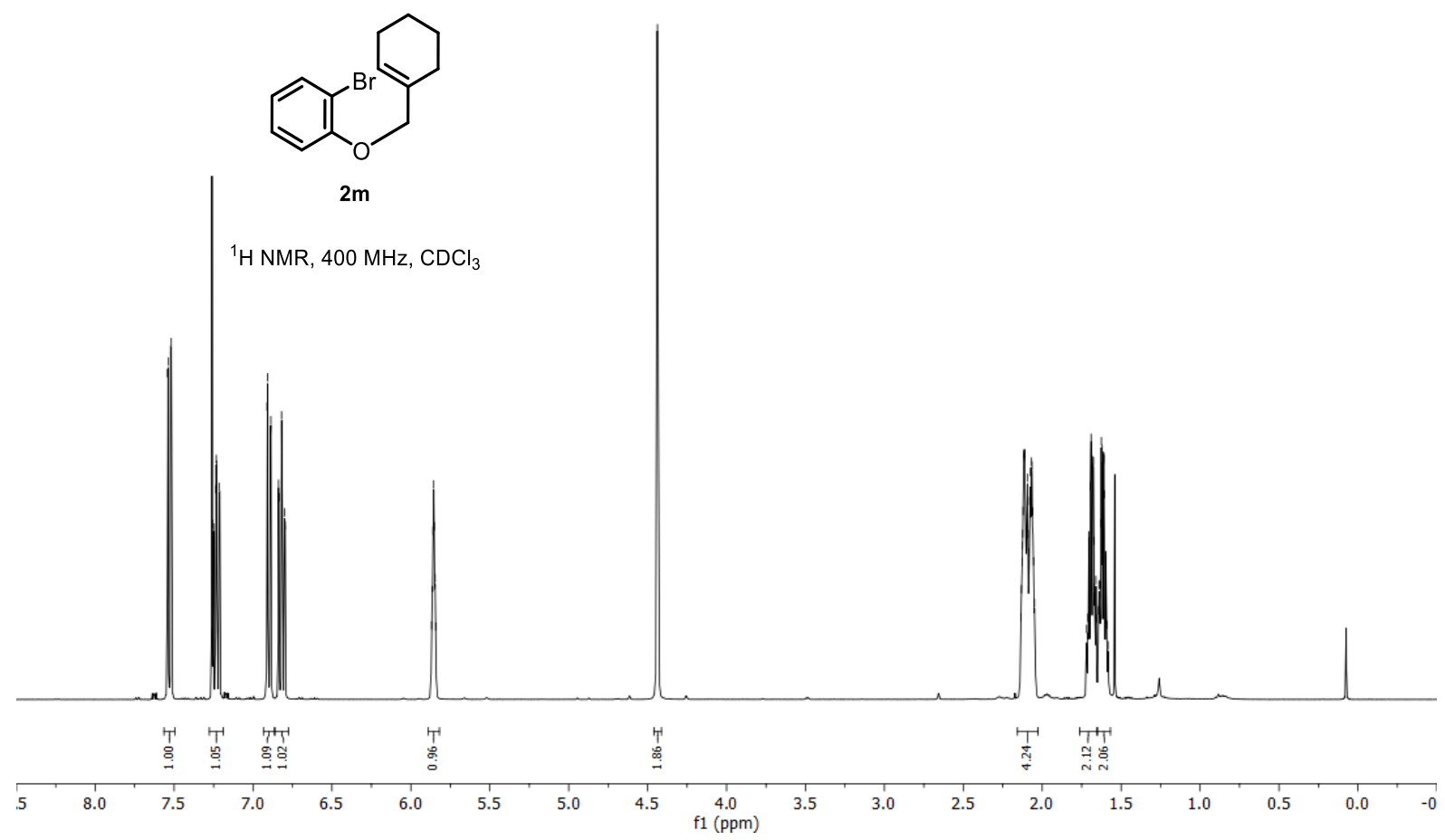

站

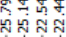
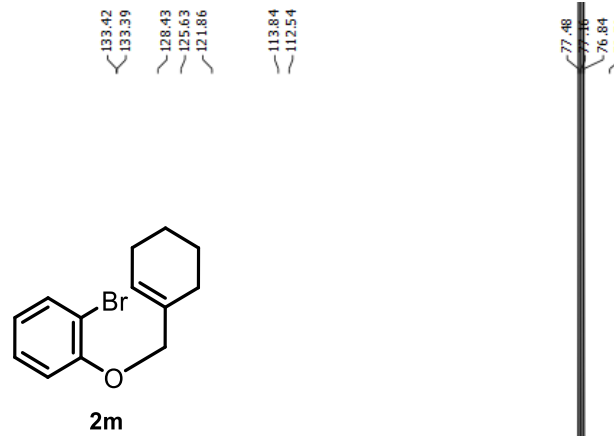

${ }^{13} \mathrm{C}$ NMR, $101 \mathrm{MHz}, \mathrm{CDCl}_{3}$

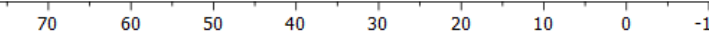


<smiles>CC(=CCOc1ccccc1Br)CCCC(N)=O</smiles>

$2 n$

${ }^{1} \mathrm{H} \mathrm{NMR}, 400 \mathrm{MHz}, \mathrm{CDCl}_{3}$

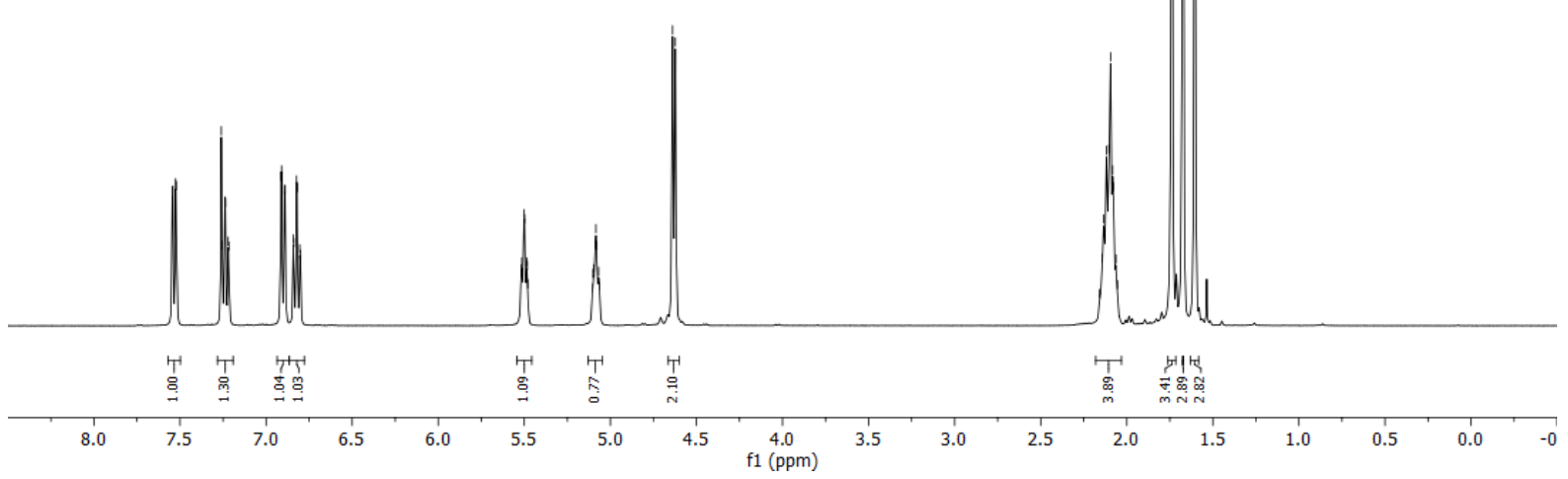

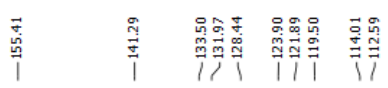<smiles>O=C(CCCC(O)=CCOc1ccccc1Br)[N+](=O)[O-]</smiles>

$2 n$

${ }^{13} \mathrm{C}$ NMR, $101 \mathrm{MHz}, \mathrm{CDCl}_{3}$

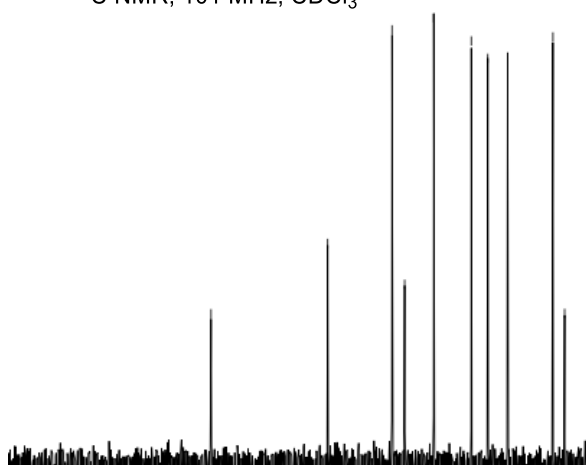

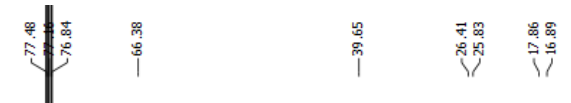

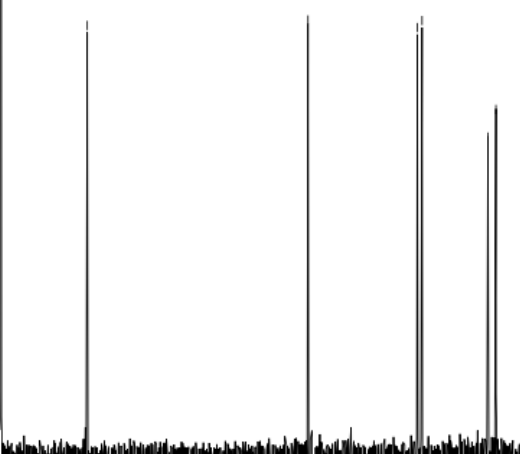

10

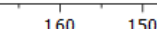

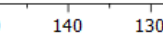

90 f1 (ppm) 


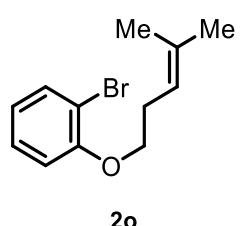

${ }^{1} \mathrm{H} \mathrm{NMR}, 400 \mathrm{MHz}, \mathrm{CDCl}_{3}$
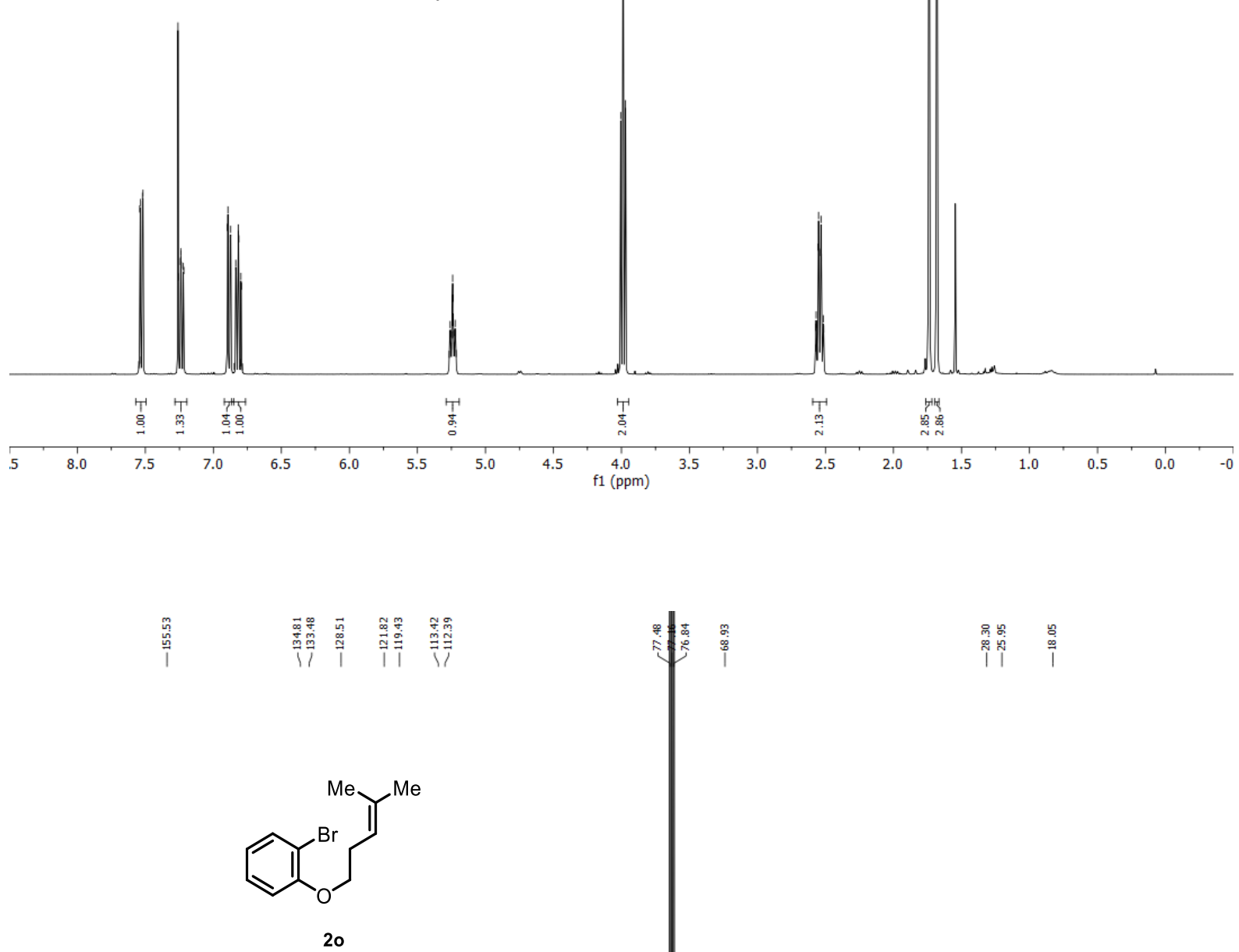

${ }^{13} \mathrm{C} \mathrm{NMR}, 101 \mathrm{MHz}, \mathrm{CDCl}_{3}$

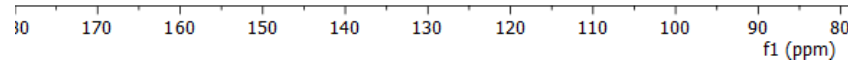

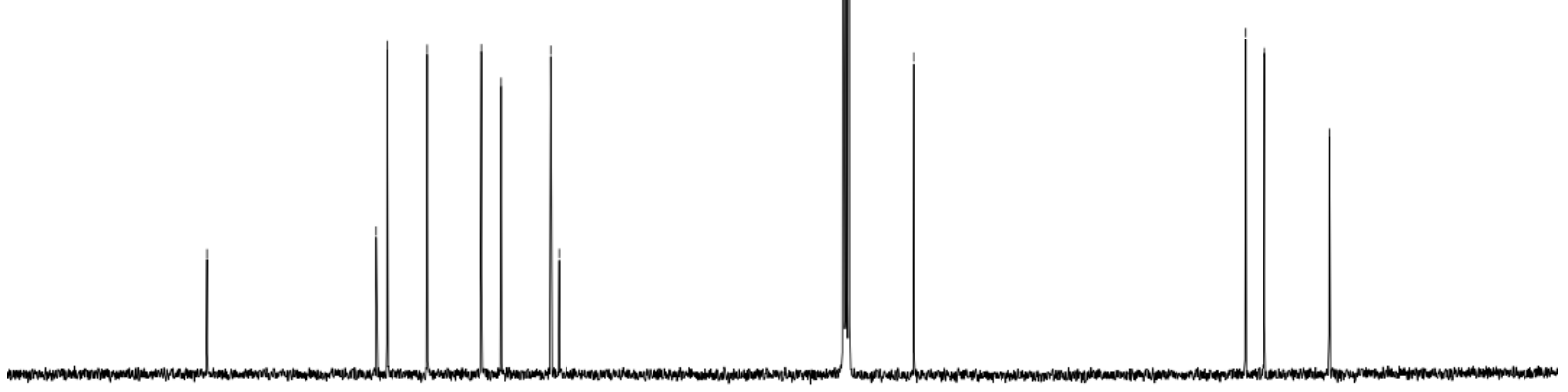




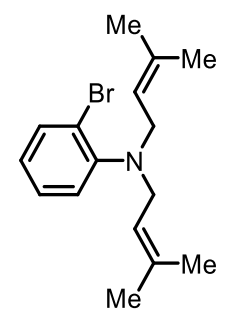

$2 q$

${ }^{1} \mathrm{H} \mathrm{NMR}, 400 \mathrm{MHz}, \mathrm{CDCl}_{3}$

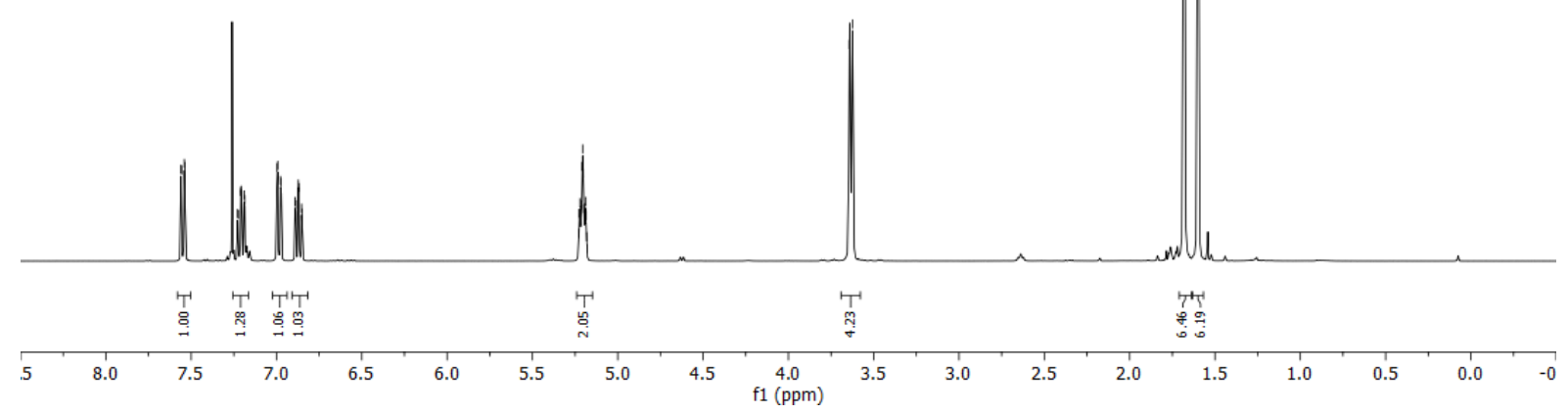

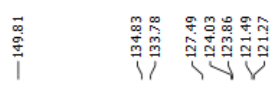

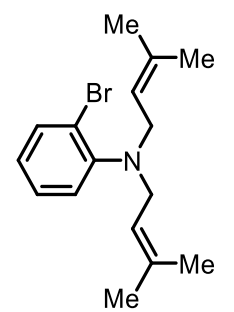

$2 q$

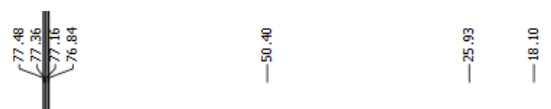

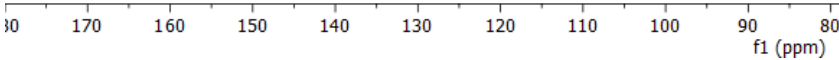

${ }^{13} \mathrm{C} \mathrm{NMR}, 101 \mathrm{MHz}, \mathrm{CDCl}_{3}$

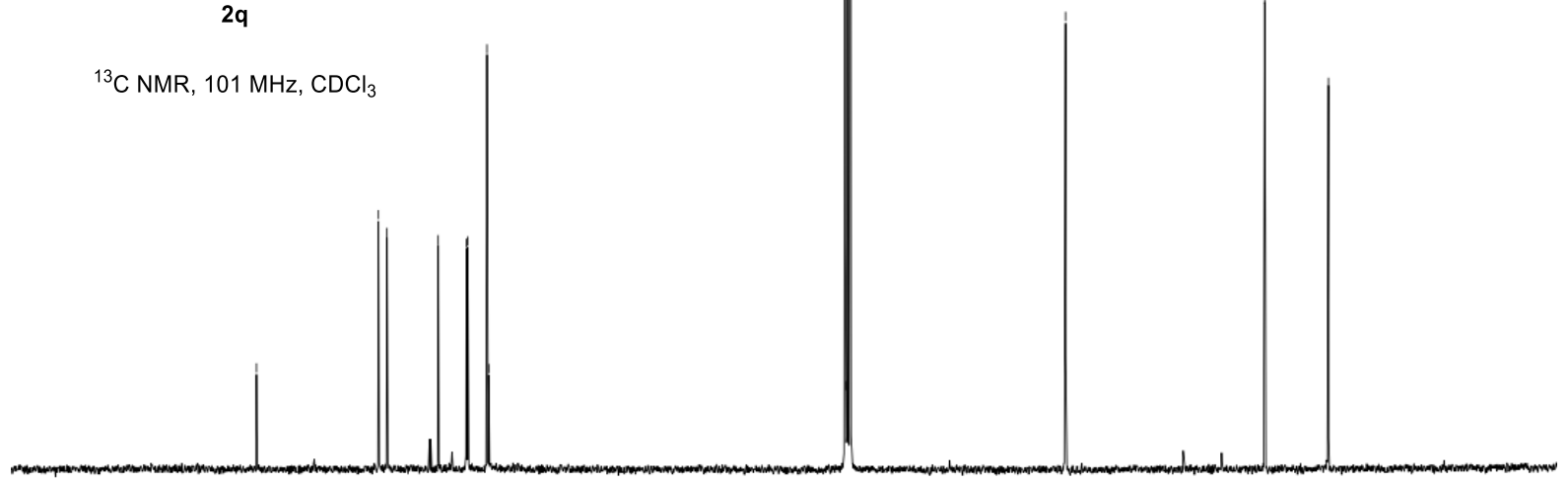




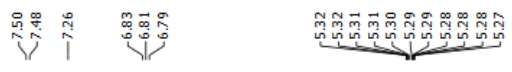

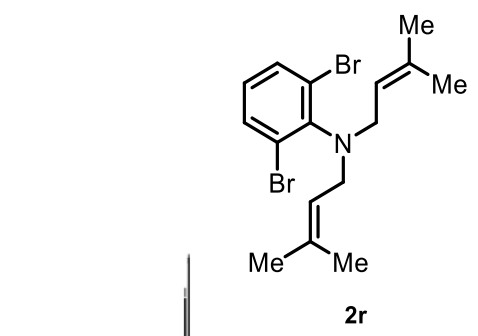

${ }^{1} \mathrm{H} \mathrm{NMR}, 400 \mathrm{MHz}, \mathrm{CDCl}_{3}$

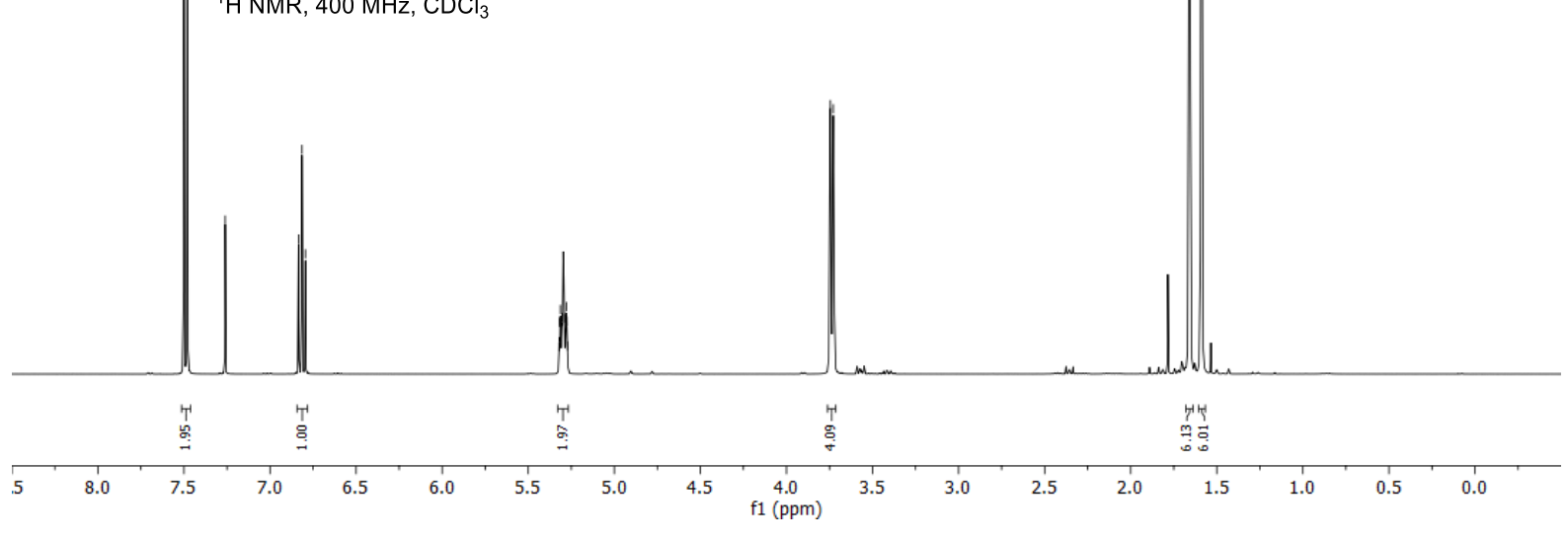

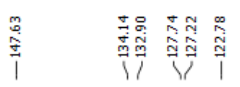

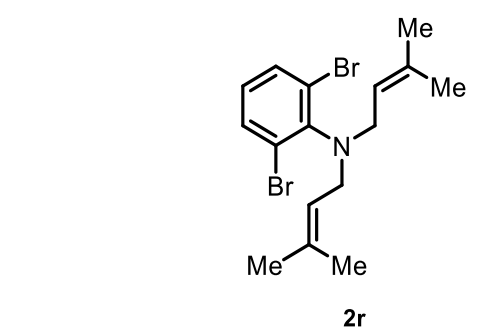

${ }^{13} \mathrm{C}$ NMR, $101 \mathrm{MHz}, \mathrm{CDCl}_{3}$

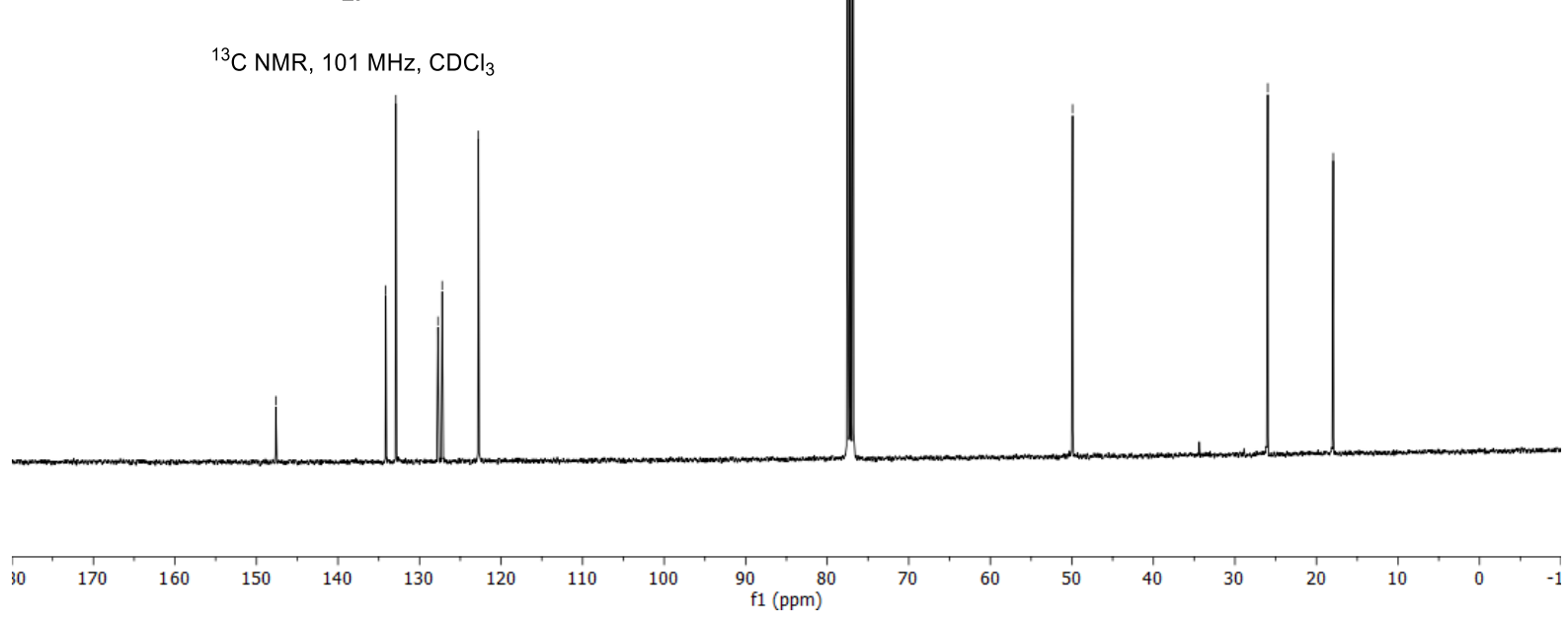

跍

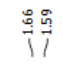

88 

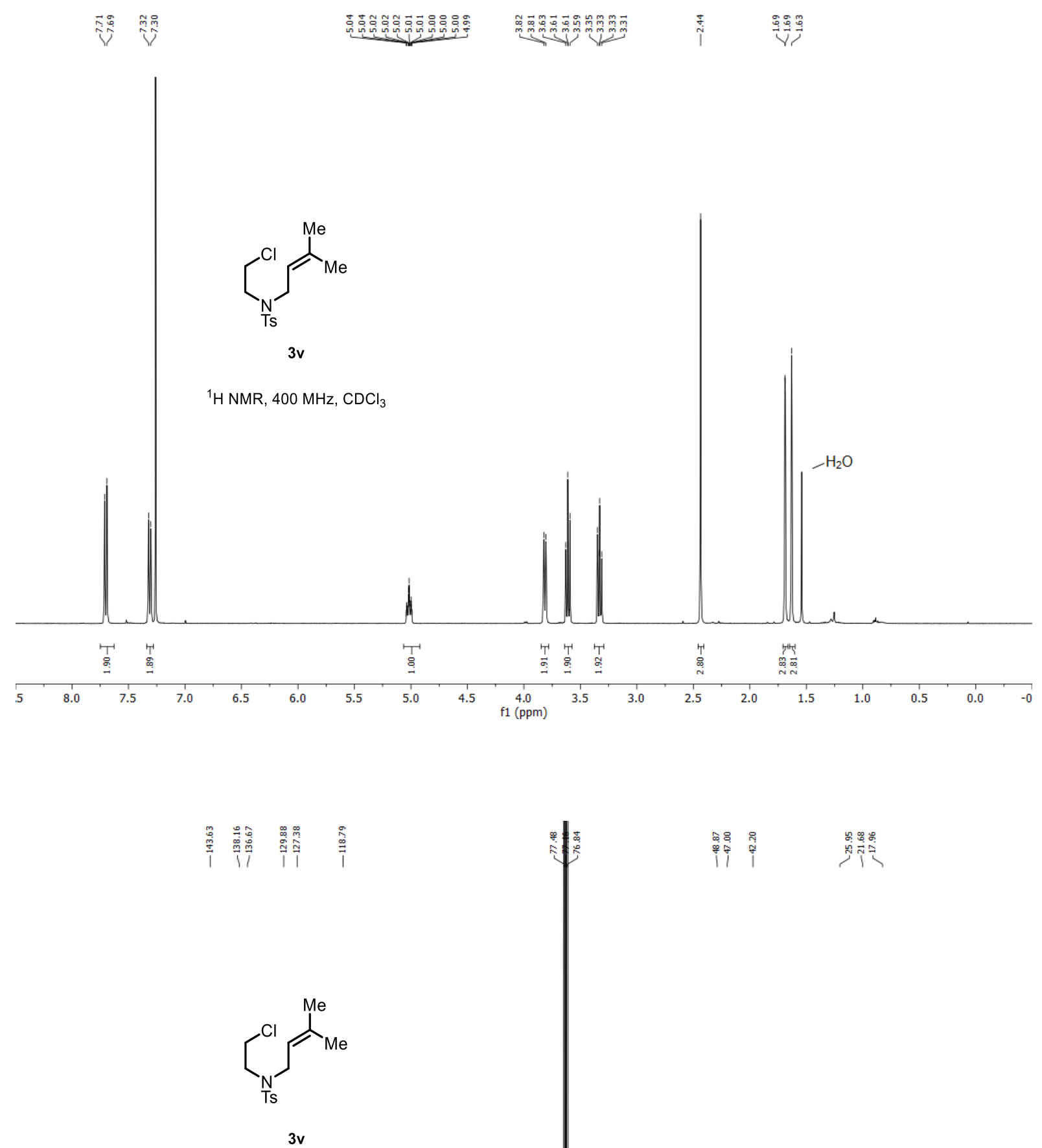

${ }^{13} \mathrm{C} \mathrm{NMR}, 101 \mathrm{MHz}, \mathrm{CDCl}_{3}$
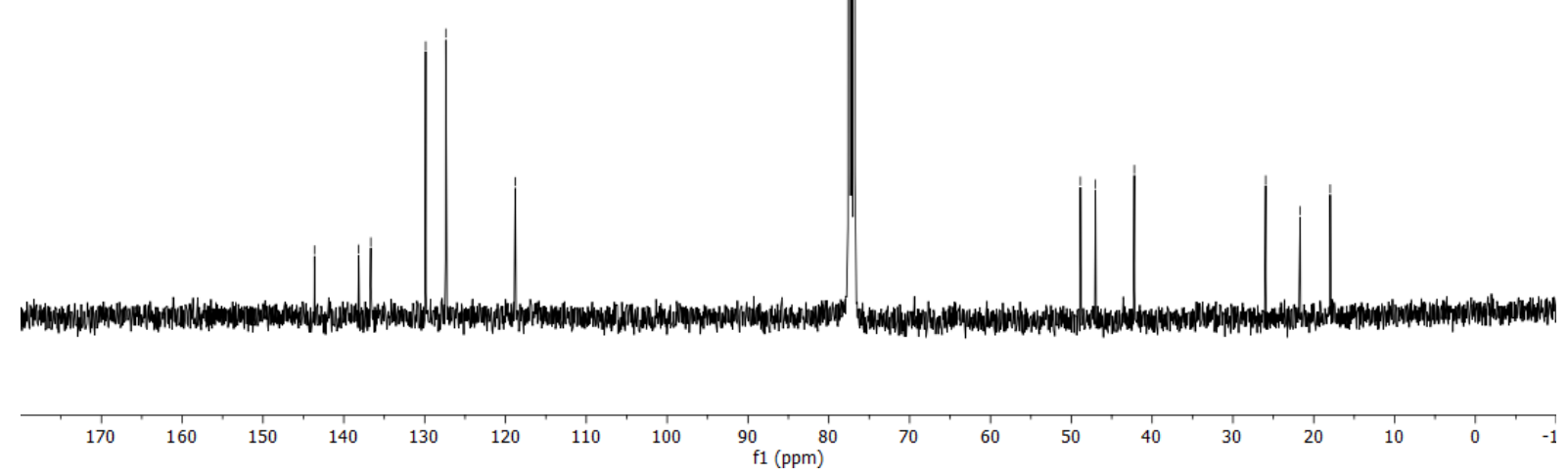


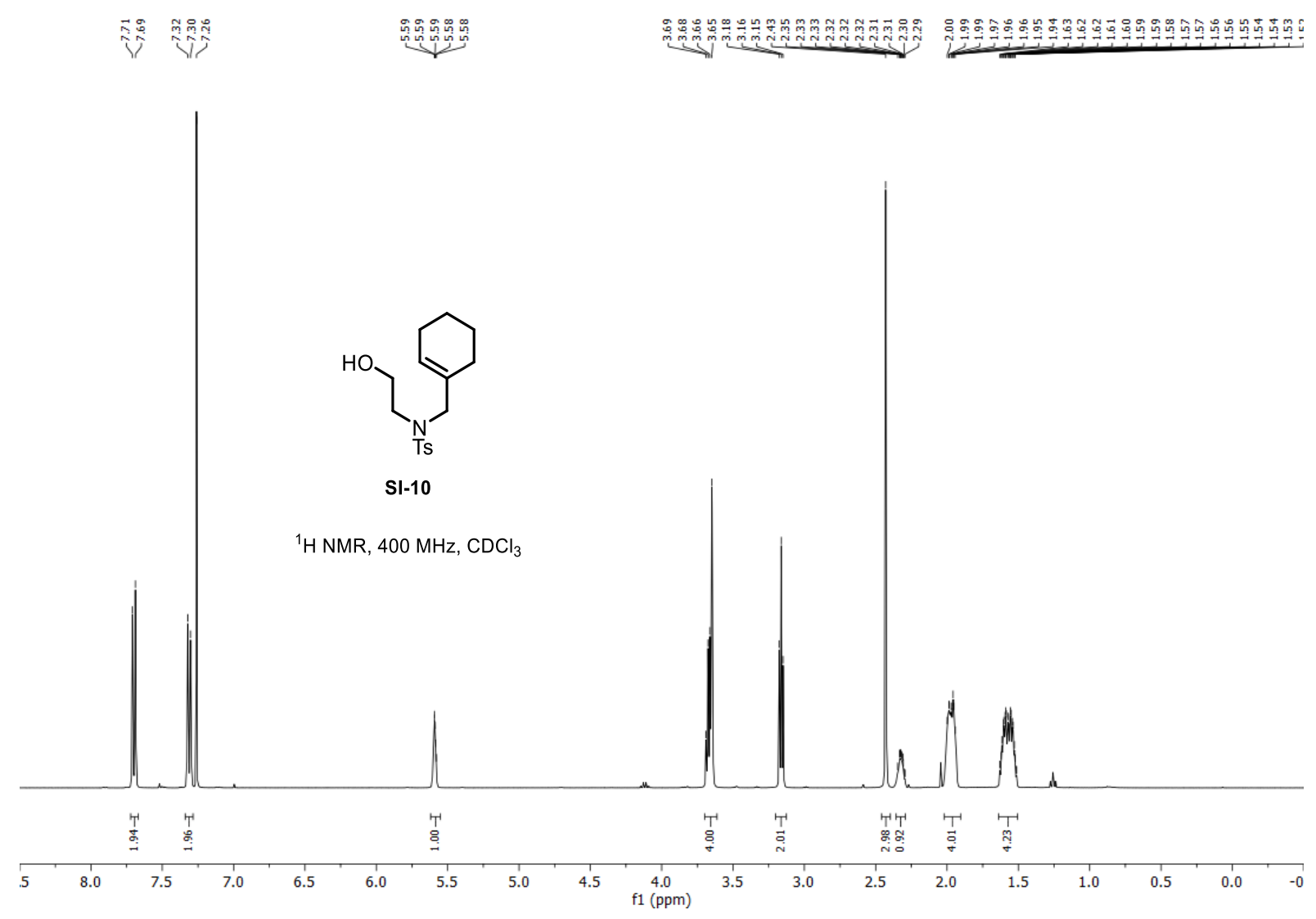

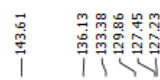

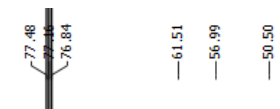

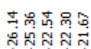

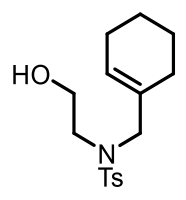

SI-10

${ }^{13} \mathrm{C} \mathrm{NMR}, 101 \mathrm{MHz}, \mathrm{CDCl}_{3}$

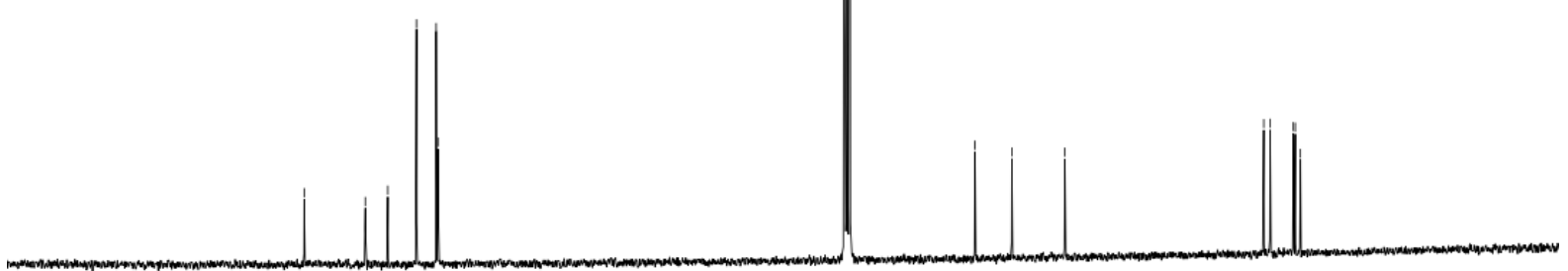

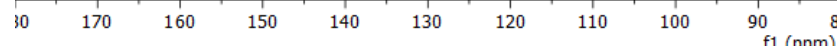

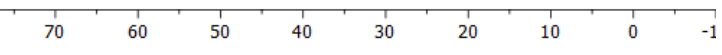




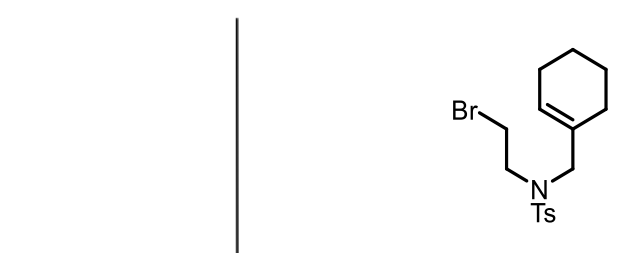

$2 \mathrm{x}$

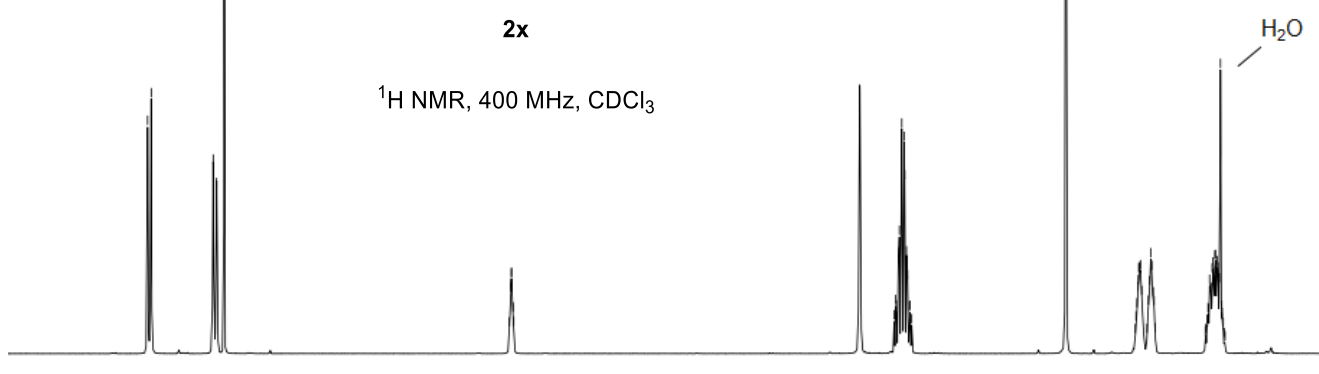

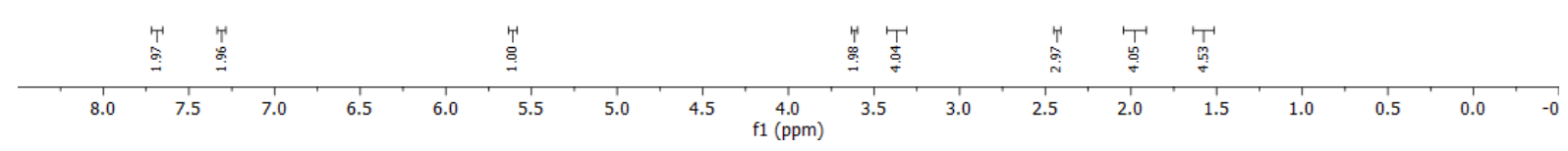

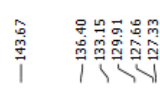

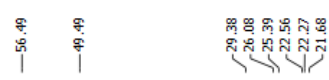

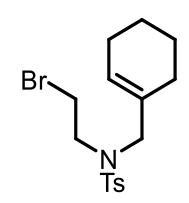

$2 x$

${ }^{13} \mathrm{C}$ NMR, $101 \mathrm{MHz}, \mathrm{CDCl}_{3}$

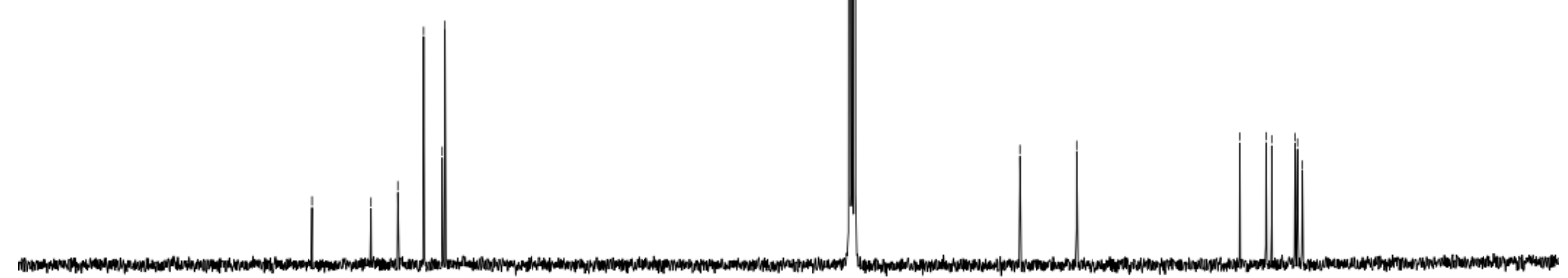

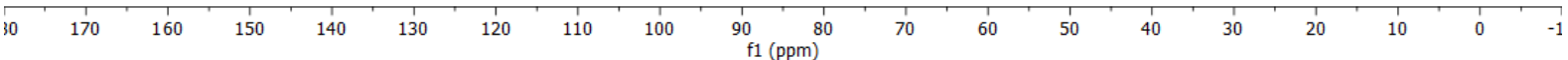




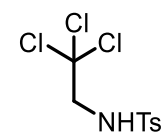

SI-13

${ }^{1} \mathrm{H}$ NMR, $400 \mathrm{MHz}, \mathrm{CDCl}_{3}$
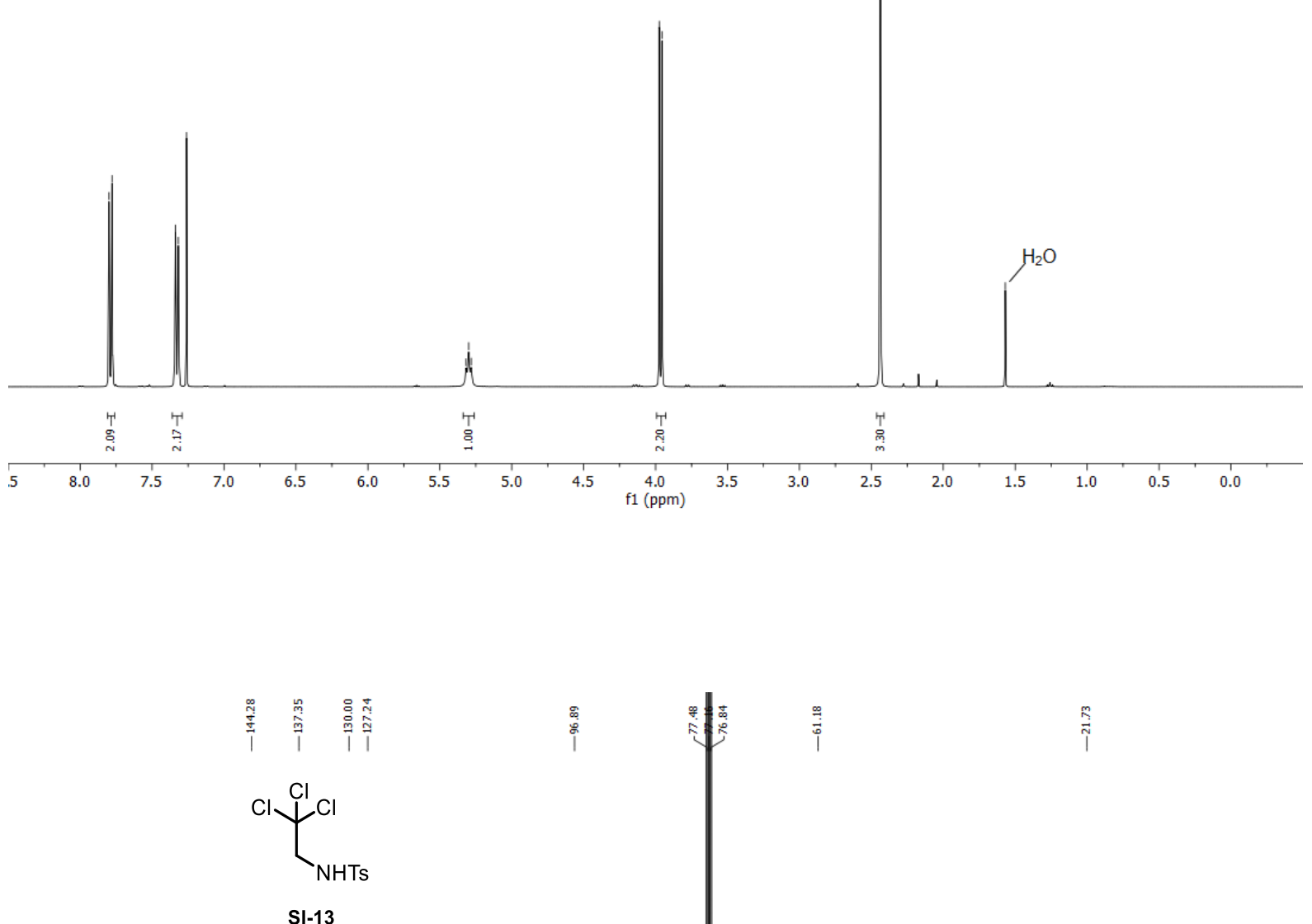

${ }^{13} \mathrm{C}$ NMR, $101 \mathrm{MHz}, \mathrm{CDCl}_{3}$

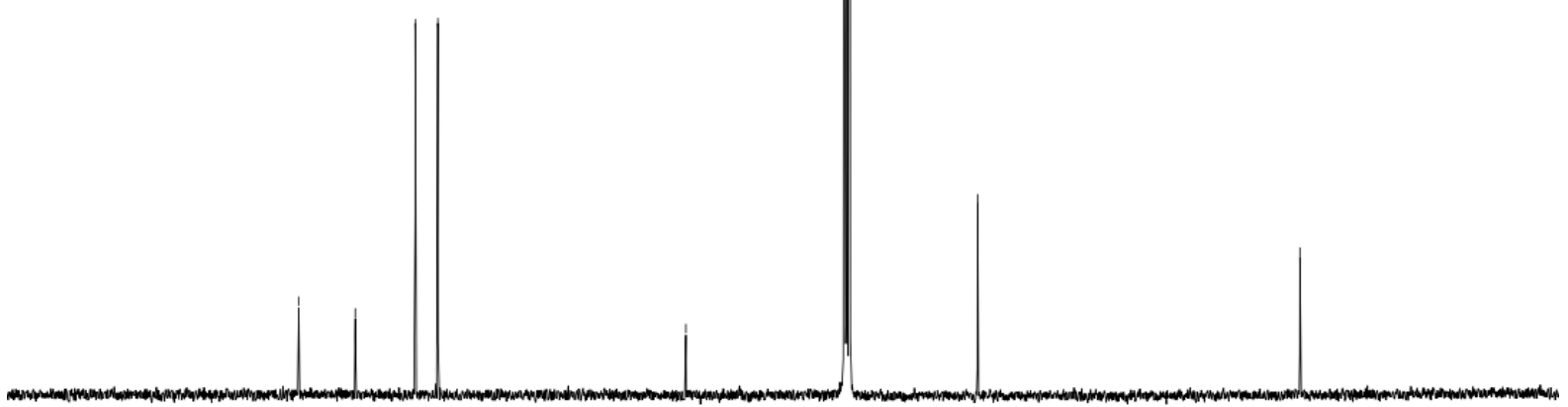

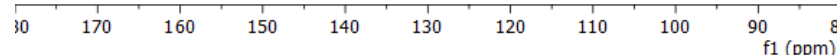




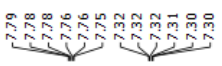

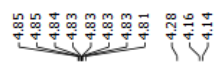

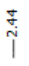

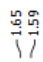

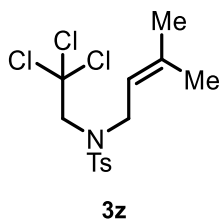

${ }^{1} \mathrm{H}$ NMR, $400 \mathrm{MHz}, \mathrm{CDCl}_{3}$
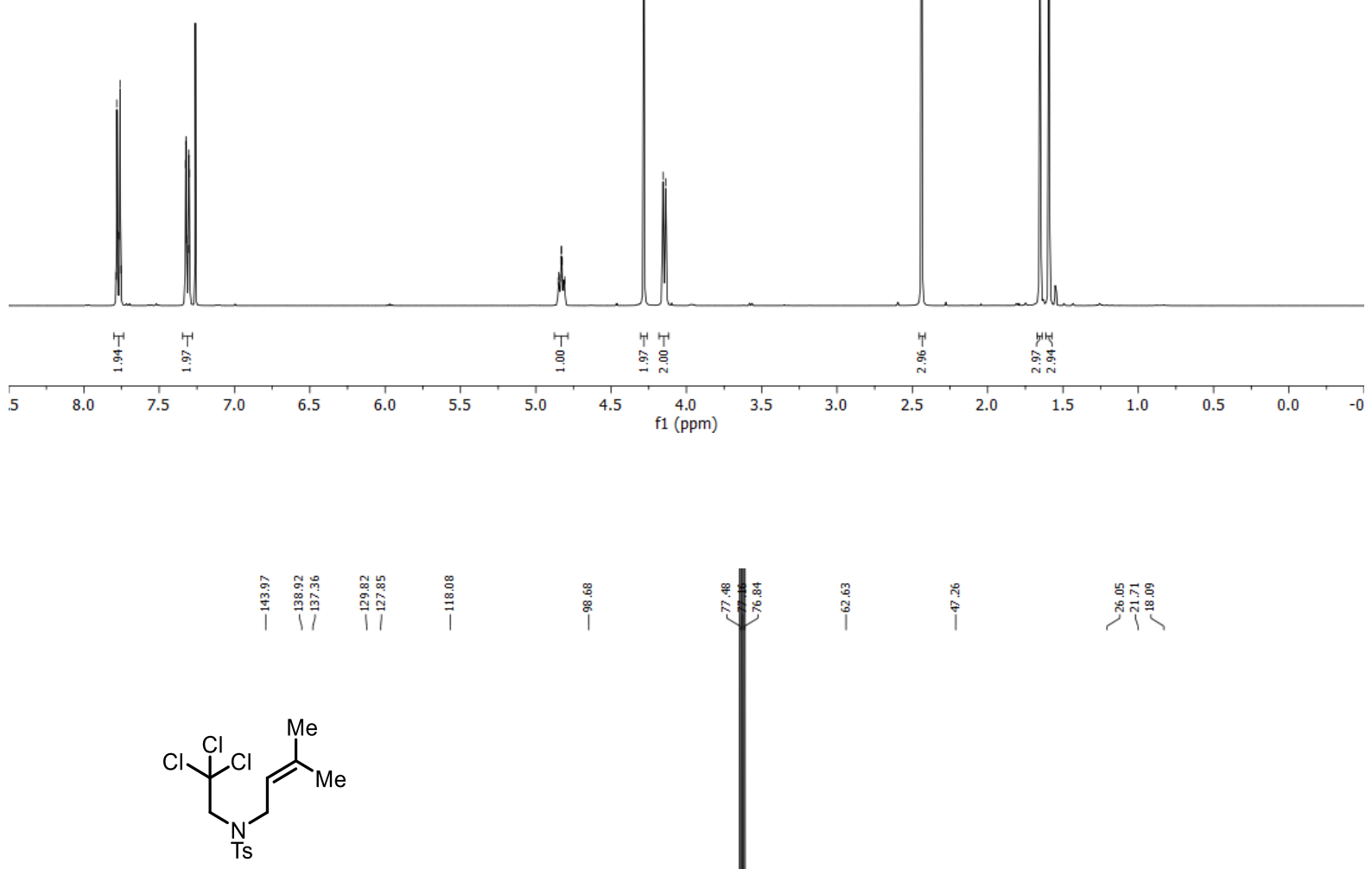

$3 z$

${ }^{13} \mathrm{C}$ NMR, $101 \mathrm{MHz}, \mathrm{CDCl}_{3}$
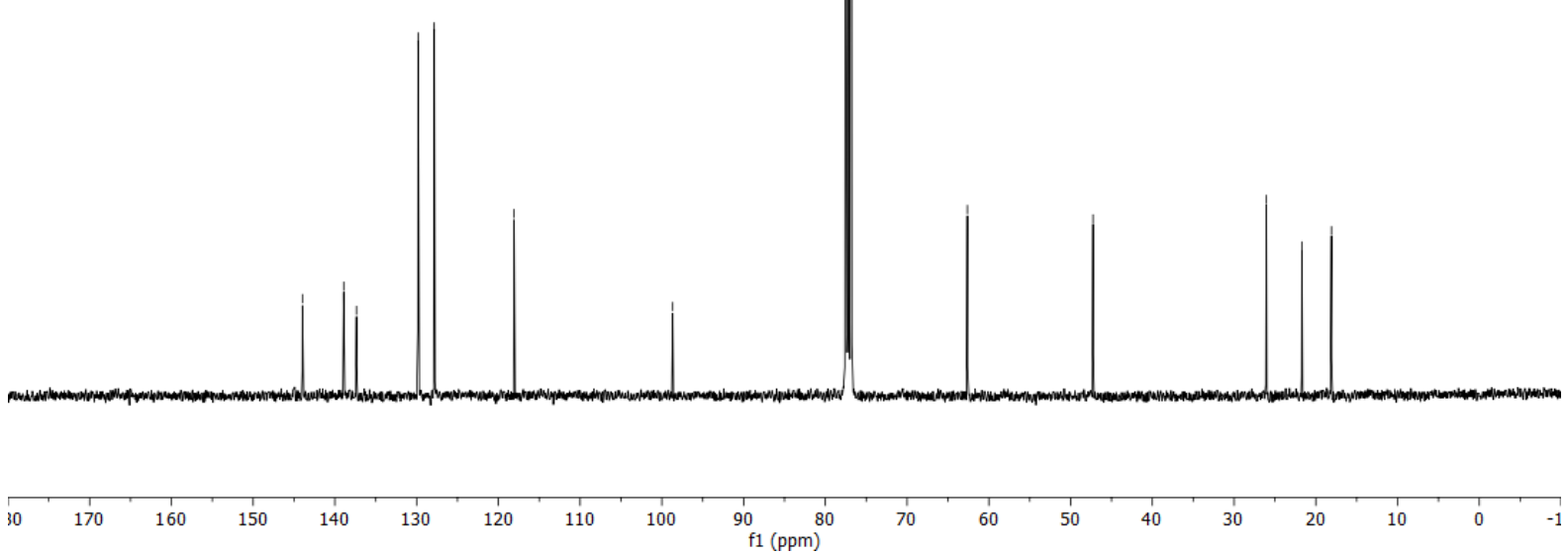

93 

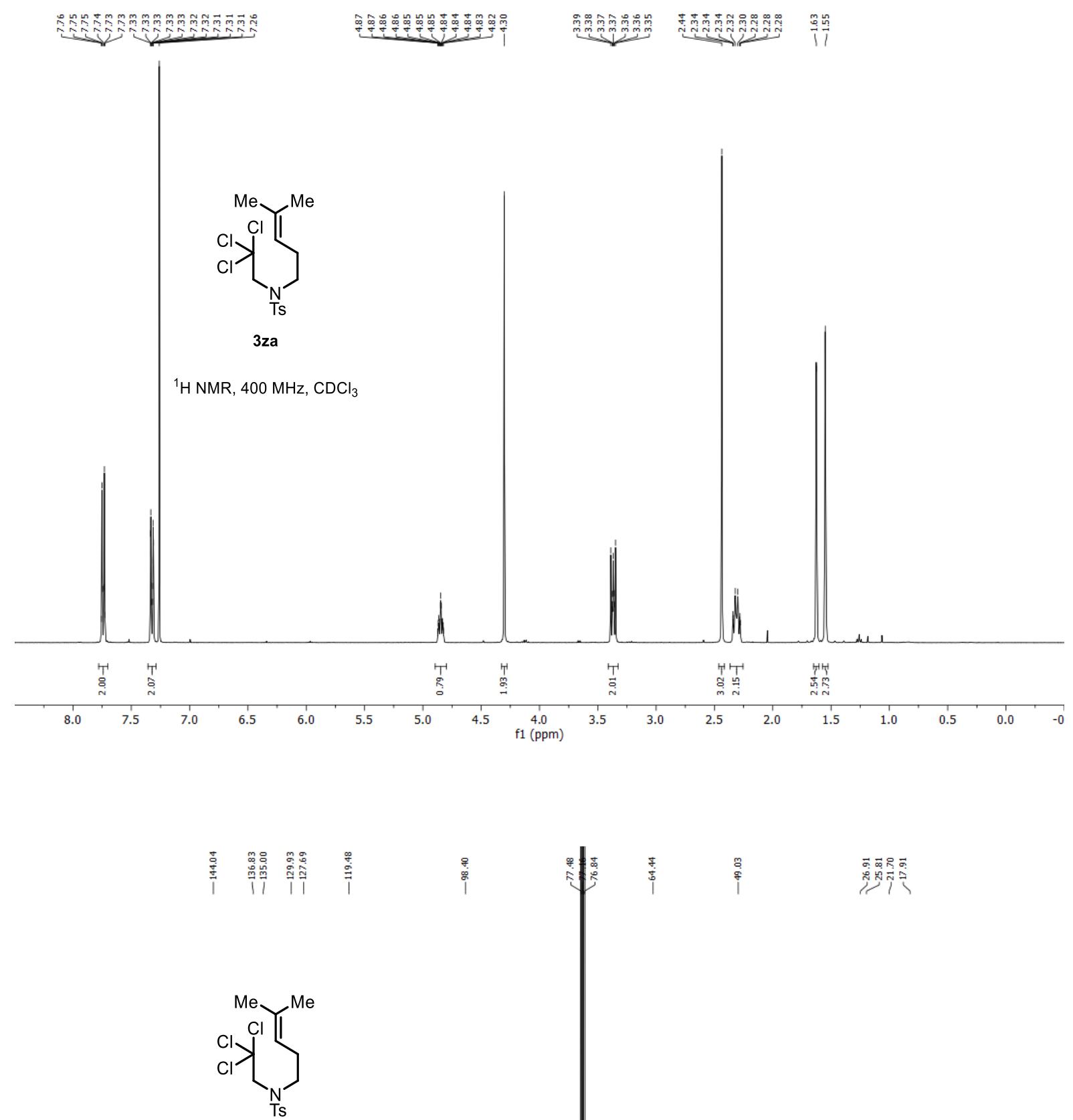

$3 z a$

${ }^{13} \mathrm{C} \mathrm{NMR}, 101 \mathrm{MHz}, \mathrm{CDCl}_{3}$

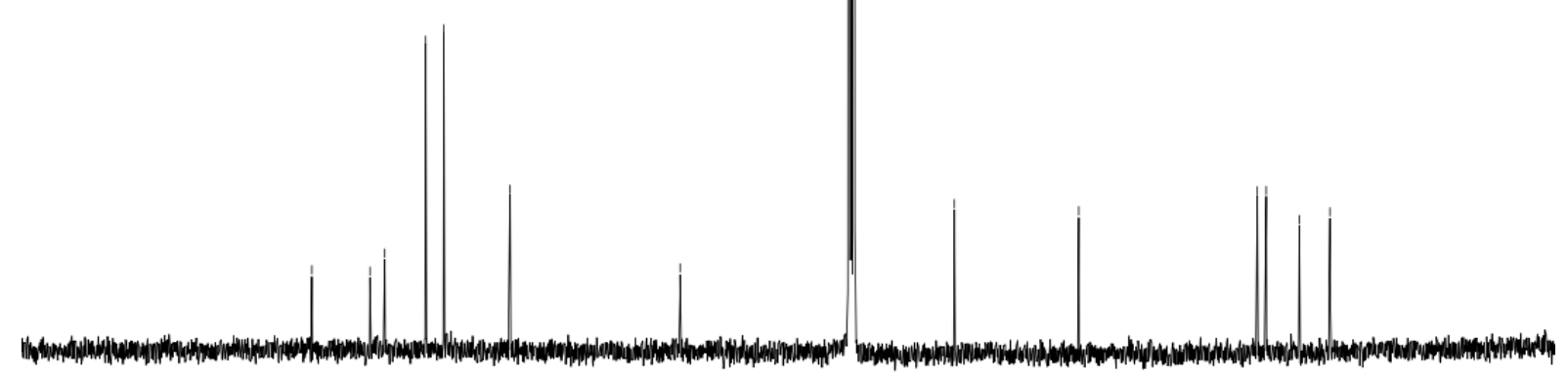

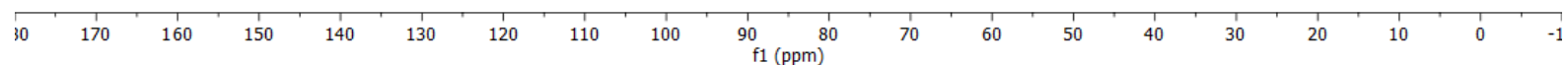




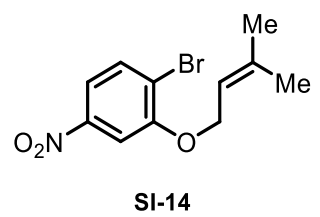

${ }^{1} \mathrm{H} \mathrm{NMR}, 400 \mathrm{MHz}, \mathrm{CDCl}_{3}$

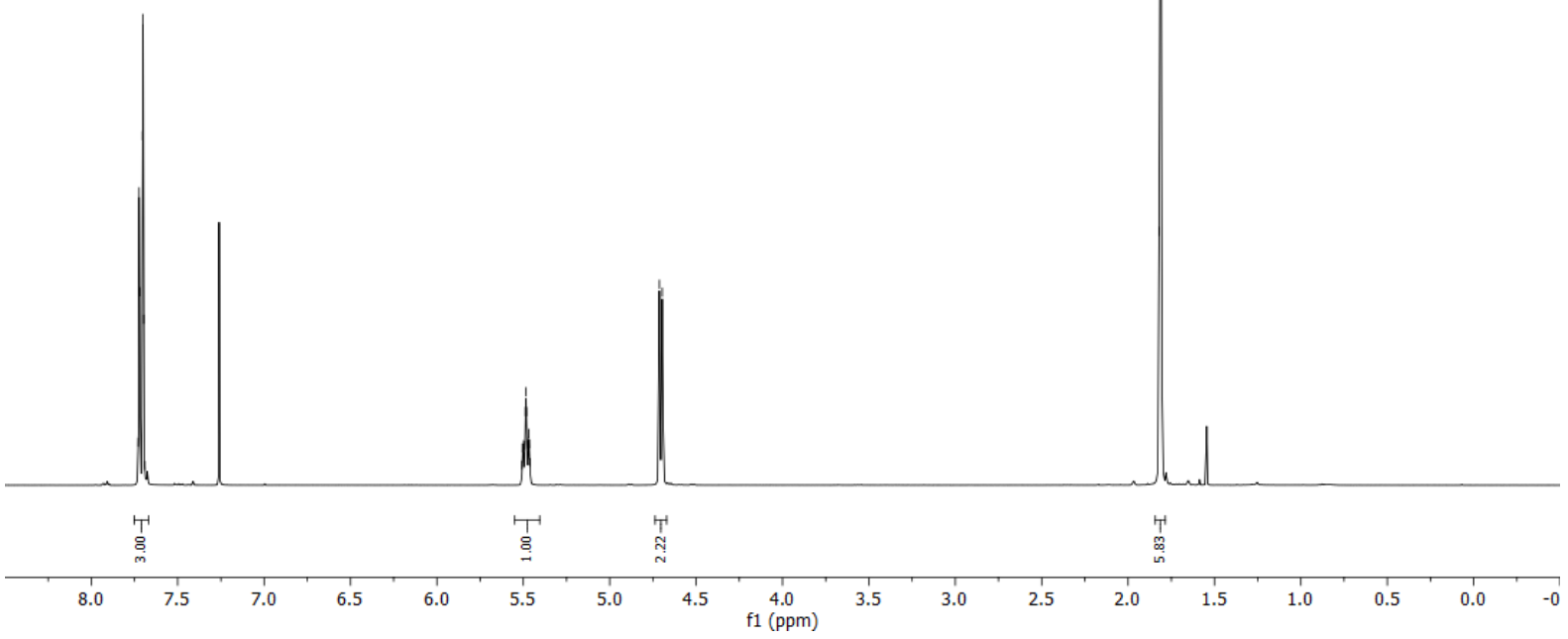

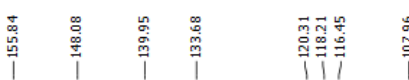

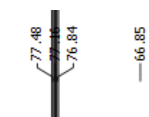

离<smiles>CC(=CCOc1cc([N+](=O)[O-])ccc1Br)[N+](=O)[O-]</smiles>

${ }^{13} \mathrm{C} \mathrm{NMR}, 101 \mathrm{MHz}, \mathrm{CDCl}_{3}$

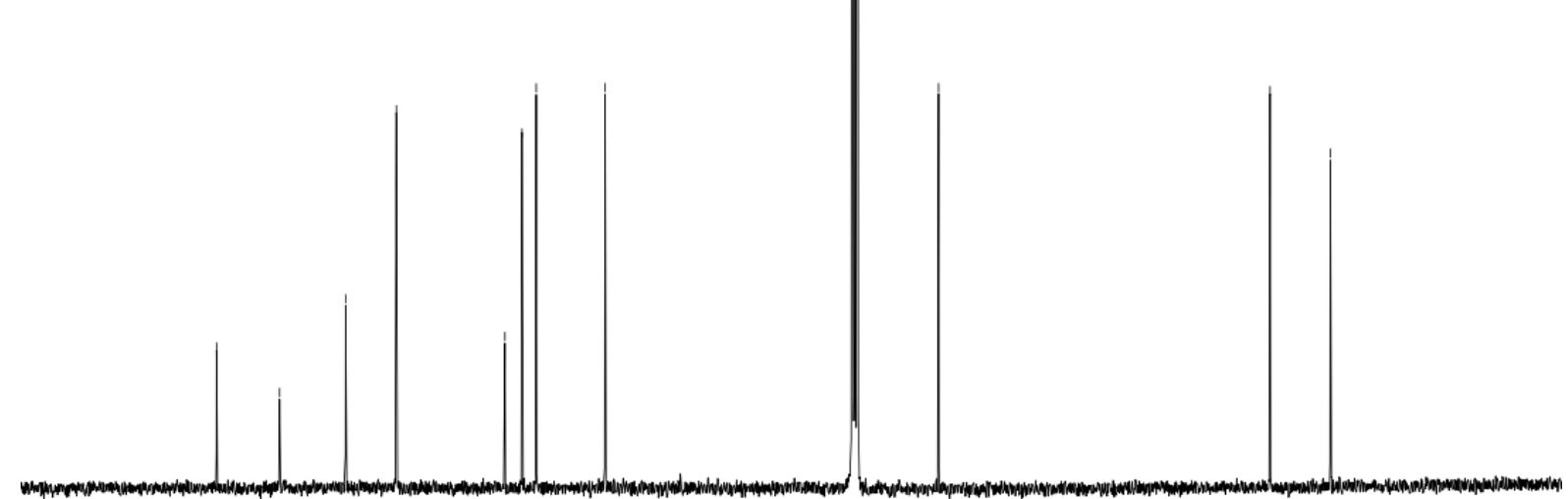

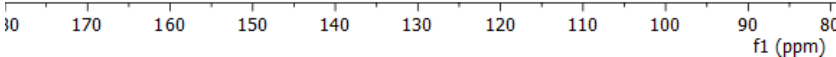




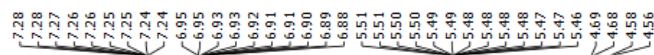

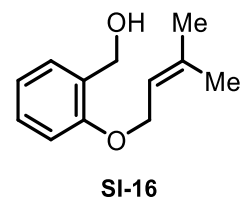

${ }^{1} \mathrm{H} \mathrm{NMR}, 400 \mathrm{MHz}, \mathrm{CDCl}_{3}$

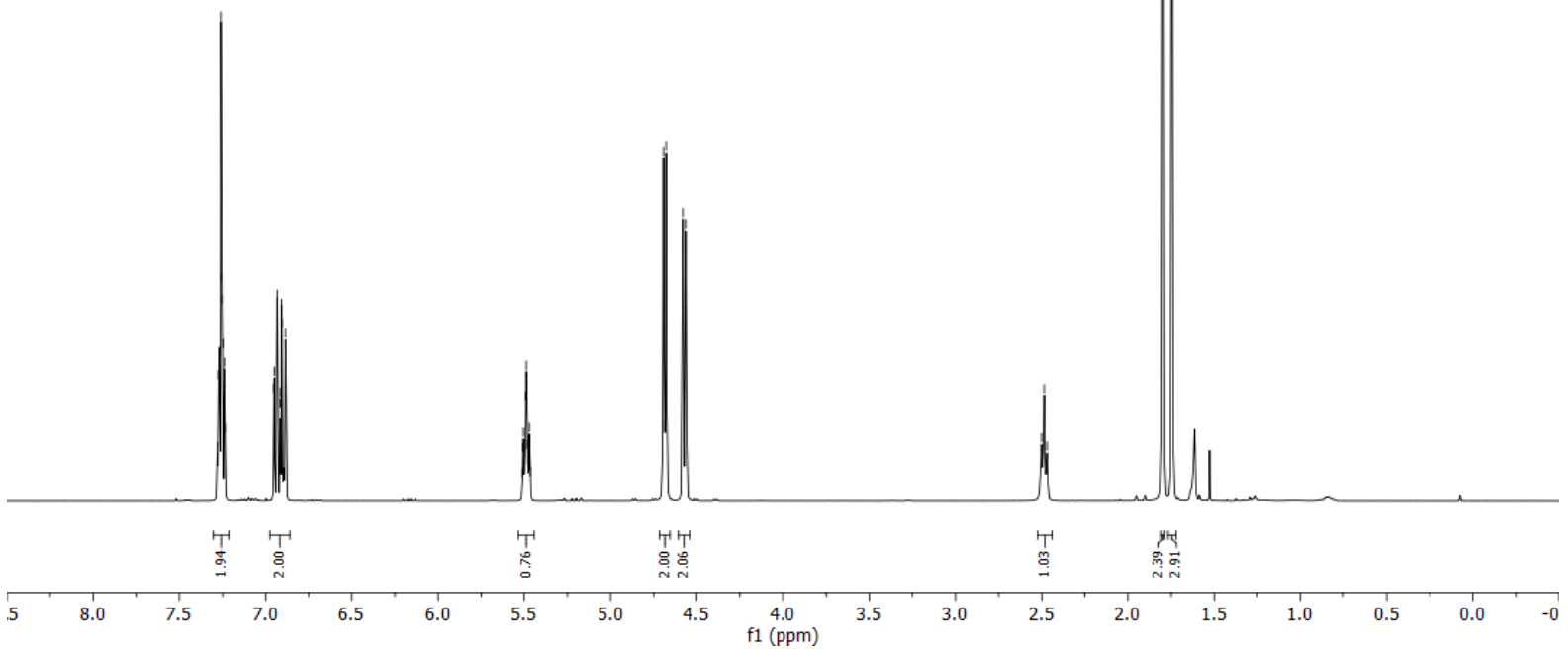

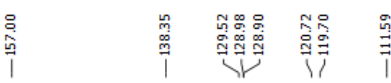

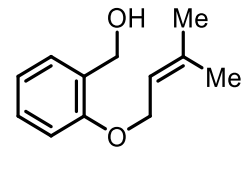

SI-16

${ }^{13} \mathrm{C}$ NMR, $101 \mathrm{MHz}, \mathrm{CDCl}_{3}$

$10-170+160+150$

$90 \quad 80$ 


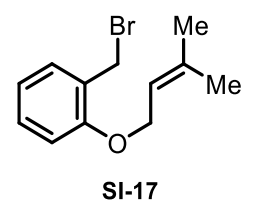

${ }^{1} \mathrm{H} \mathrm{NMR}, 400 \mathrm{MHz}, \mathrm{CDCl}_{3}$

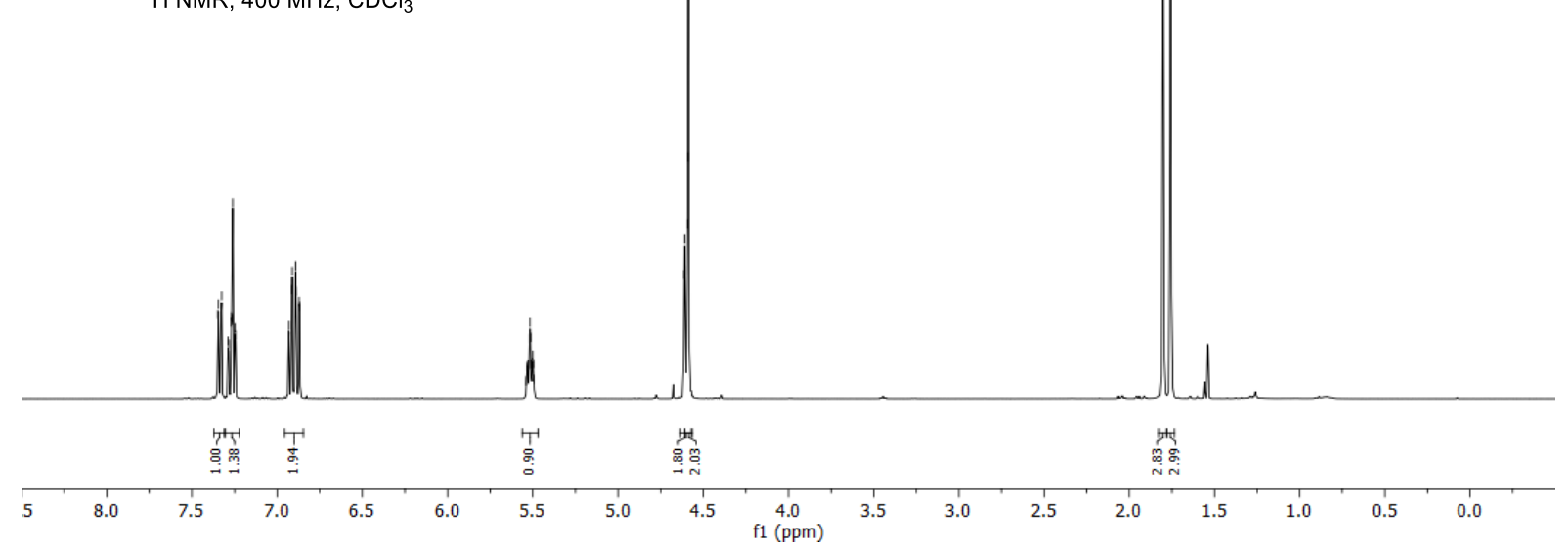

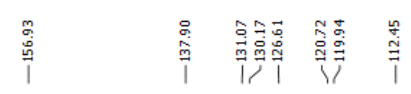

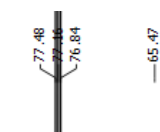

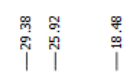

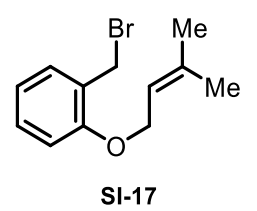

${ }^{13} \mathrm{C}$ NMR, $101 \mathrm{MHz}, \mathrm{CDCl}_{3}$

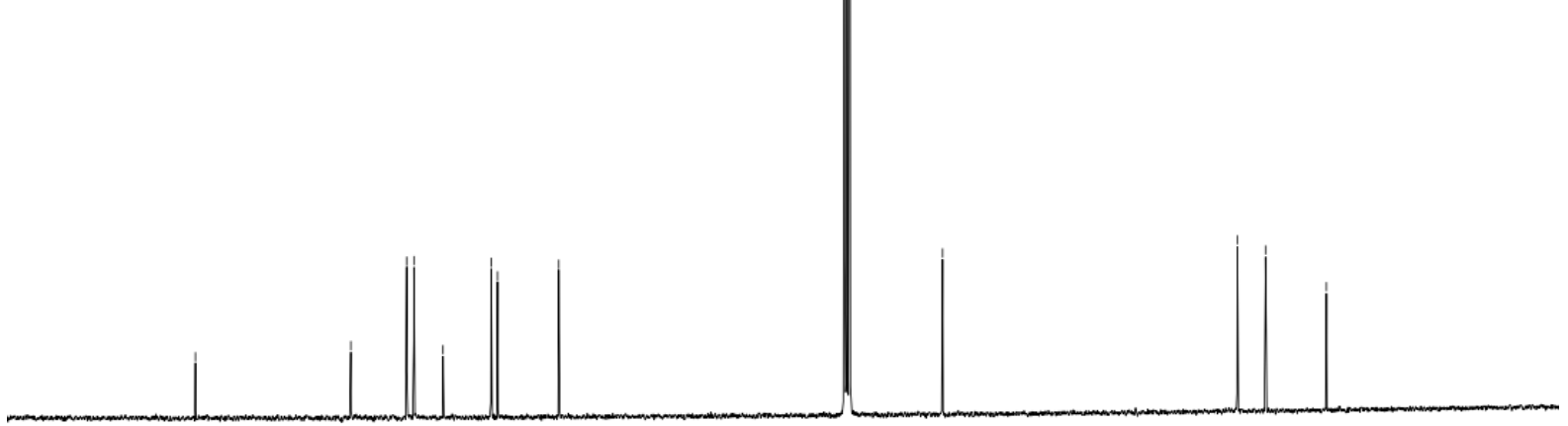

$30 \quad 170$

$160 \quad 150$

140

$120 \quad 110 \quad 100$

$90 \quad 80$ 


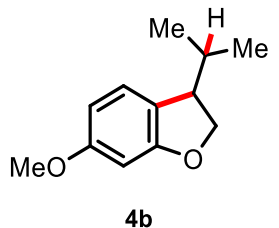

${ }^{1} \mathrm{H} \mathrm{NMR}, 400 \mathrm{MHz}, \mathrm{CDCl}_{3}$

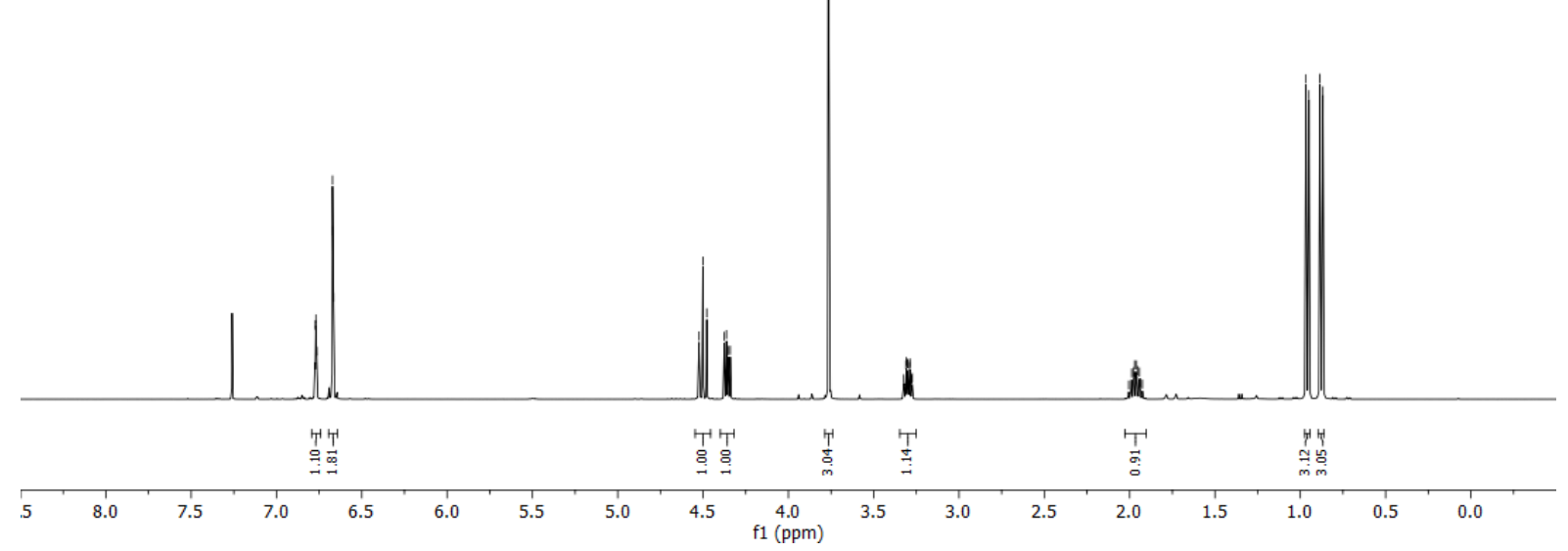

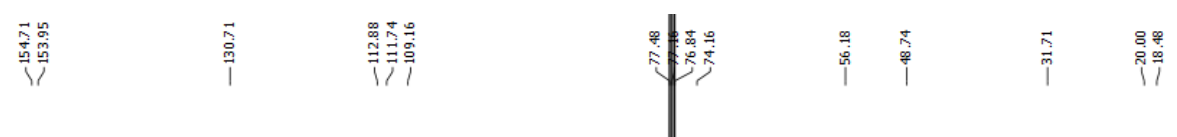

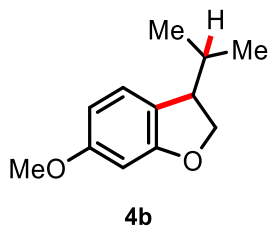

${ }^{13} \mathrm{C}$ NMR, $101 \mathrm{MHz}, \mathrm{CDCl}_{3}$

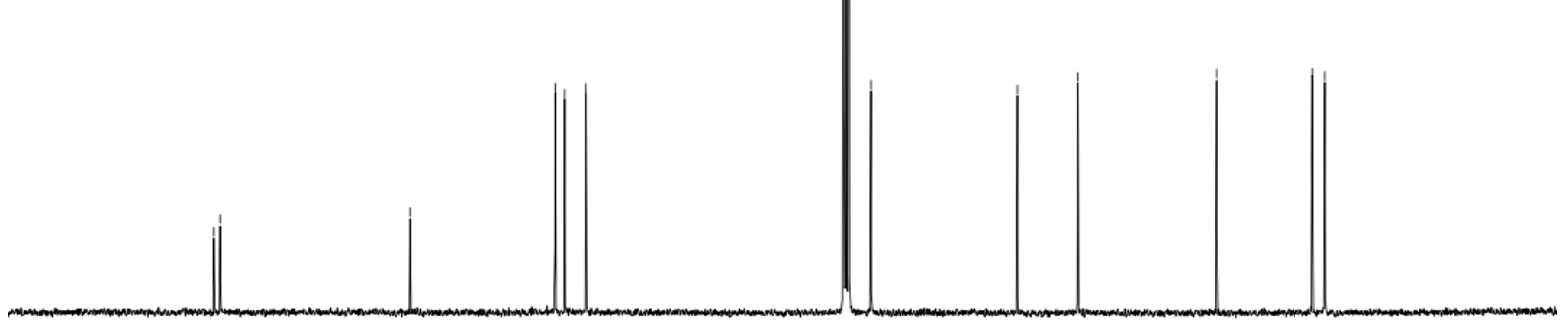

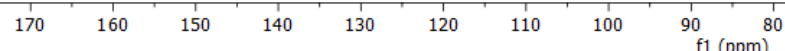

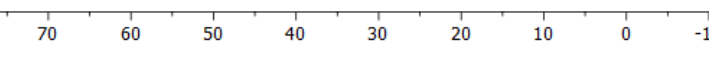




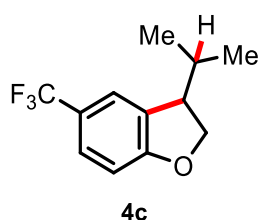

${ }^{1} \mathrm{H}$ NMR, $400 \mathrm{MHz}, \mathrm{CDCl}_{3}$
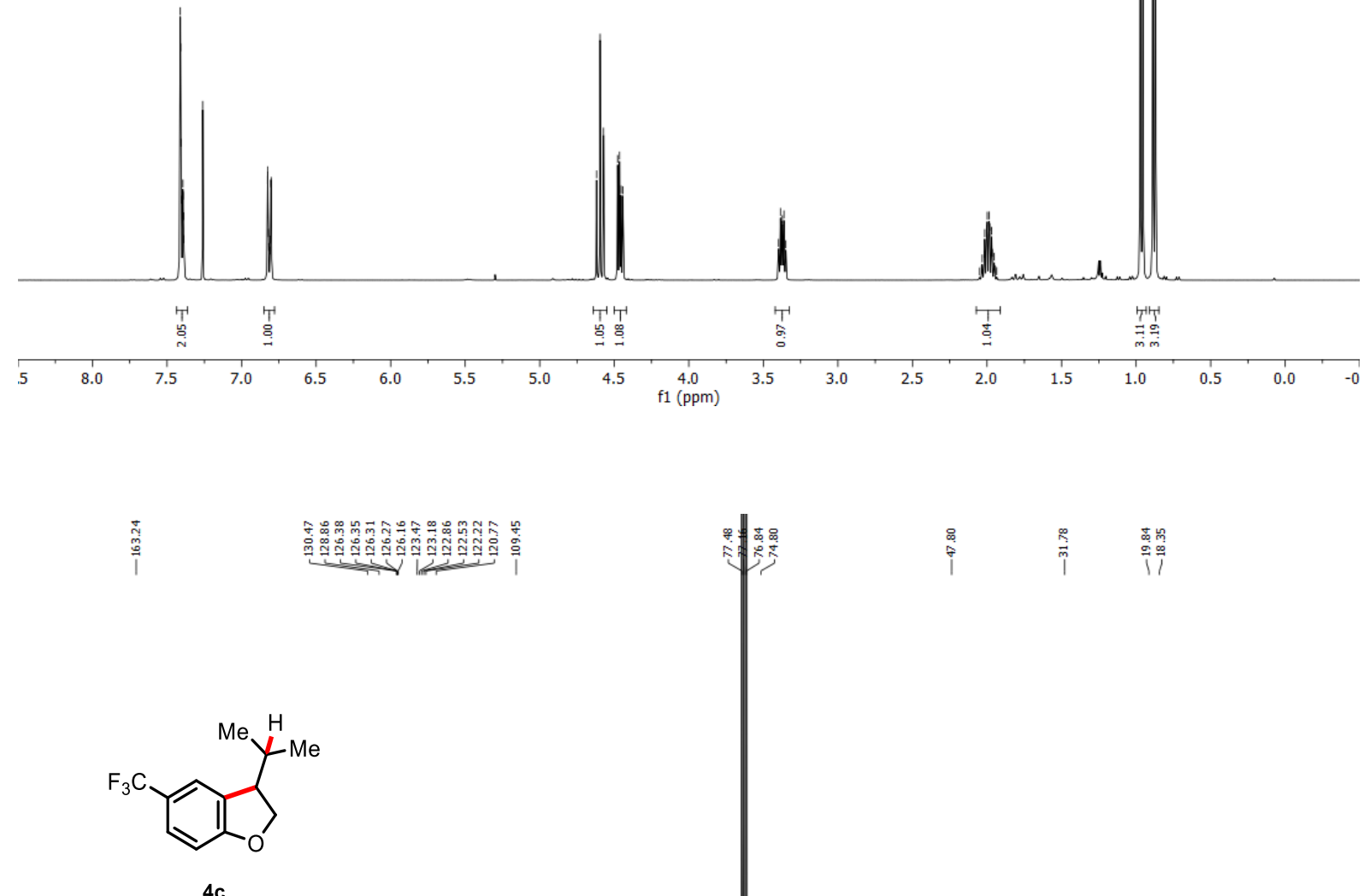

${ }^{13} \mathrm{C}$ NMR, $101 \mathrm{MHz}, \mathrm{CDCl}_{3}$

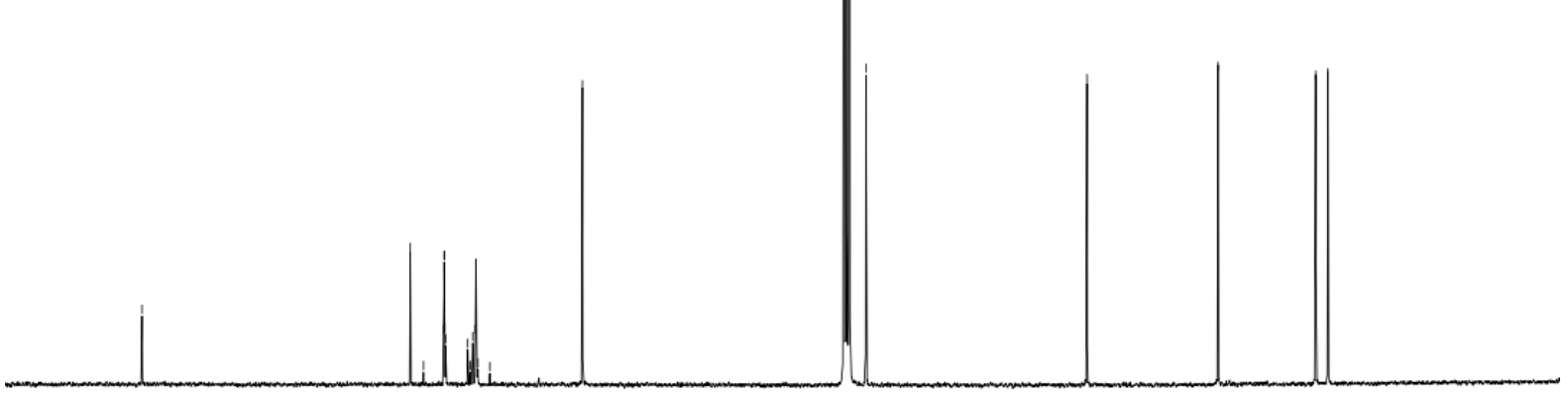

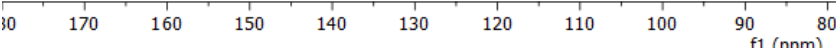




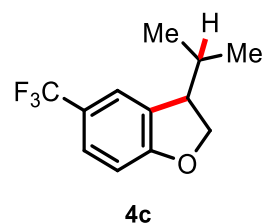

${ }^{19} \mathrm{~F} \mathrm{NMR}, 376 \mathrm{MHz}, \mathrm{CDCl}_{3}$

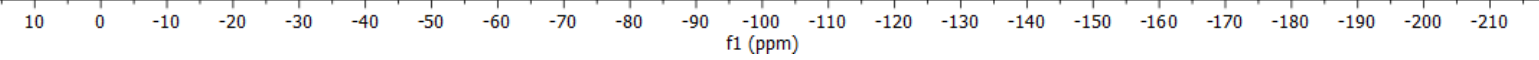




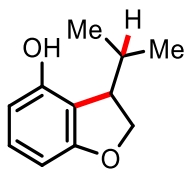

4d
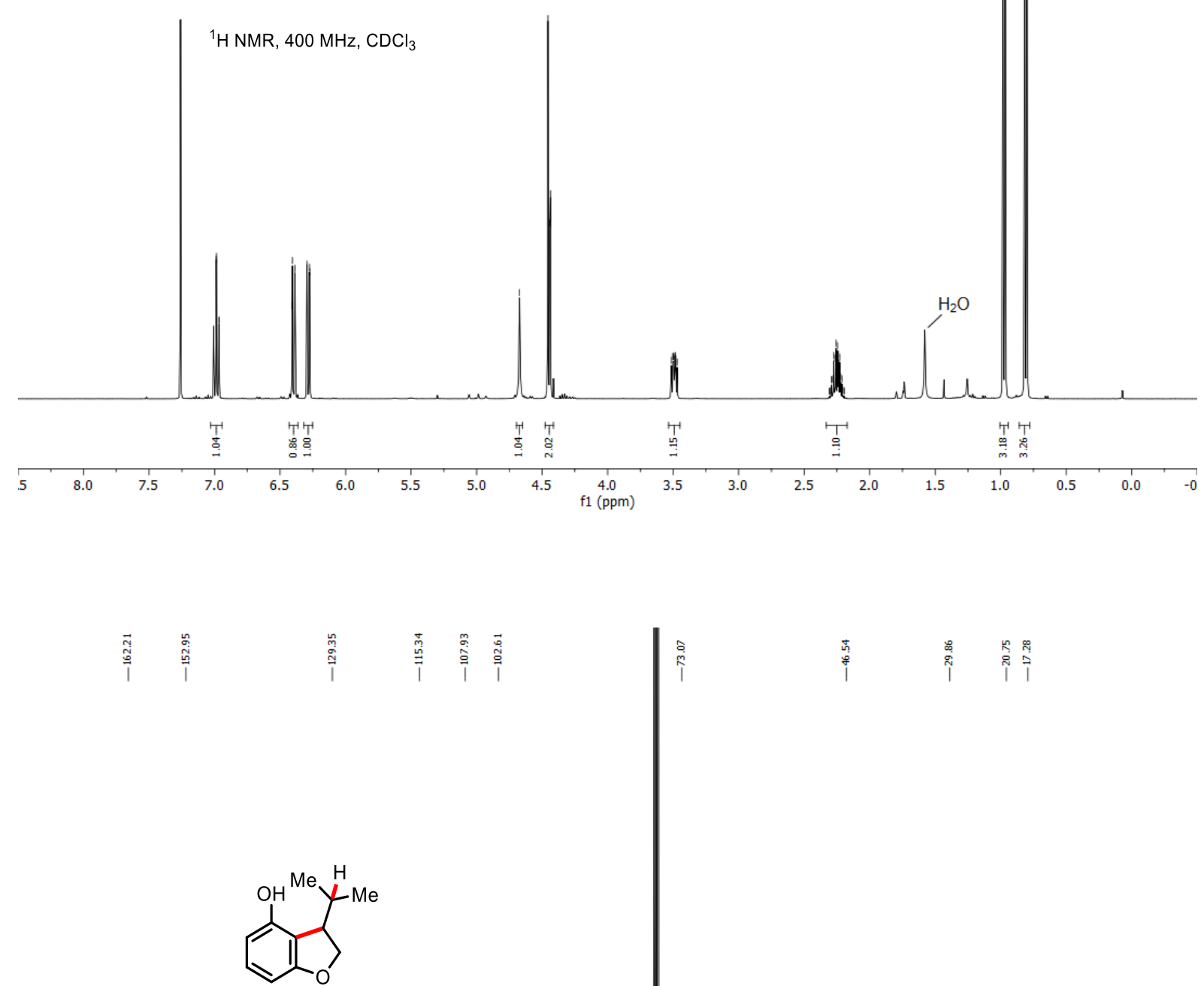

$4 d$

${ }^{13} \mathrm{C} \mathrm{NMR}, 101 \mathrm{MHz}, \mathrm{CDCl}_{3}$

\begin{tabular}{llllllllll}
\hline 10 & 170 & 160 & 150 & 140 & 130 & 120 & 110 & 100 & $\substack{\mid \\
\mathrm{f} 1(\mathrm{ppm})}$ \\
\hline
\end{tabular}
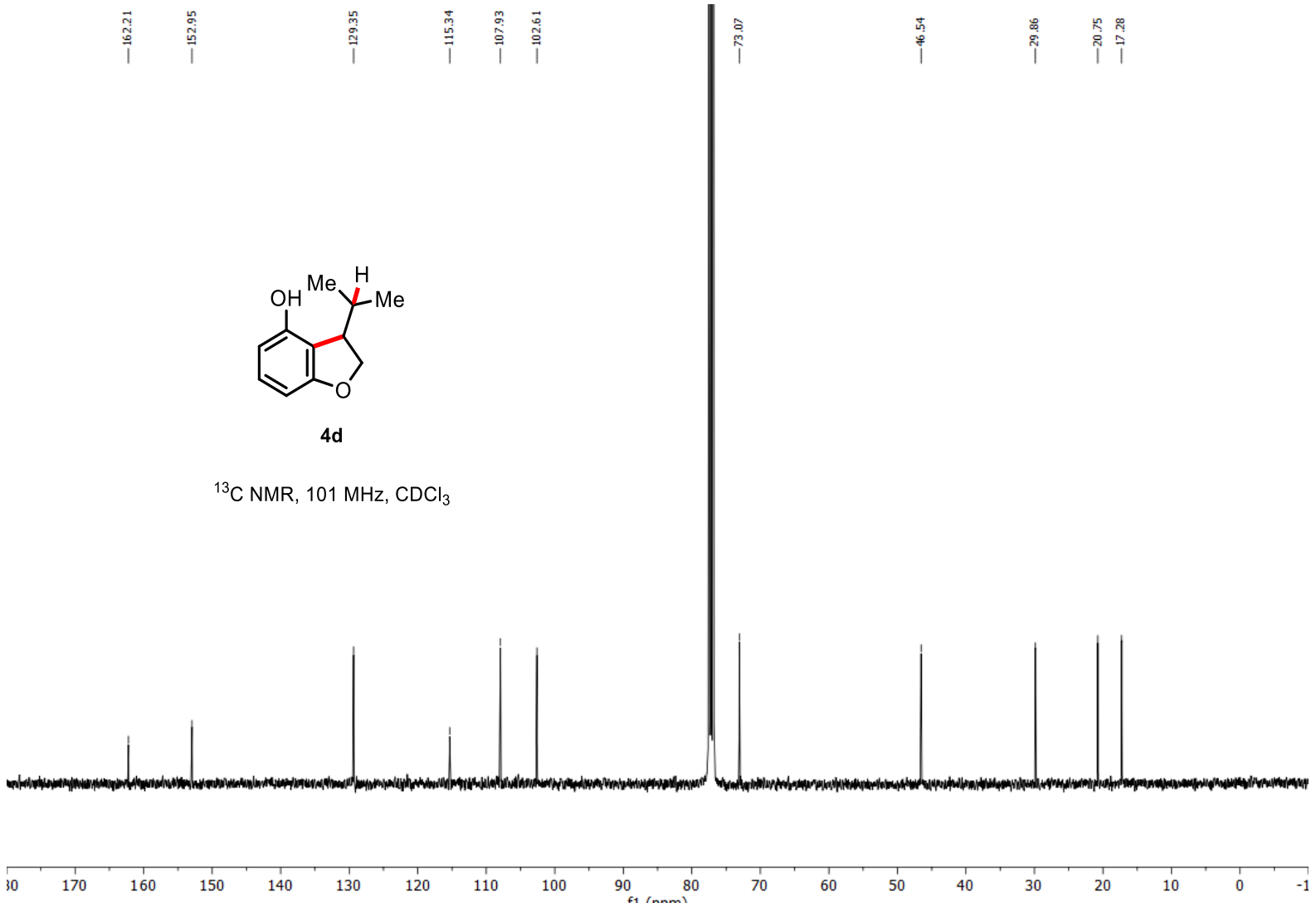


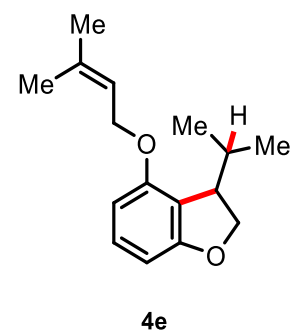

${ }^{1} \mathrm{H} \mathrm{NMR}, 400 \mathrm{MHz}, \mathrm{CDCl}_{3}$

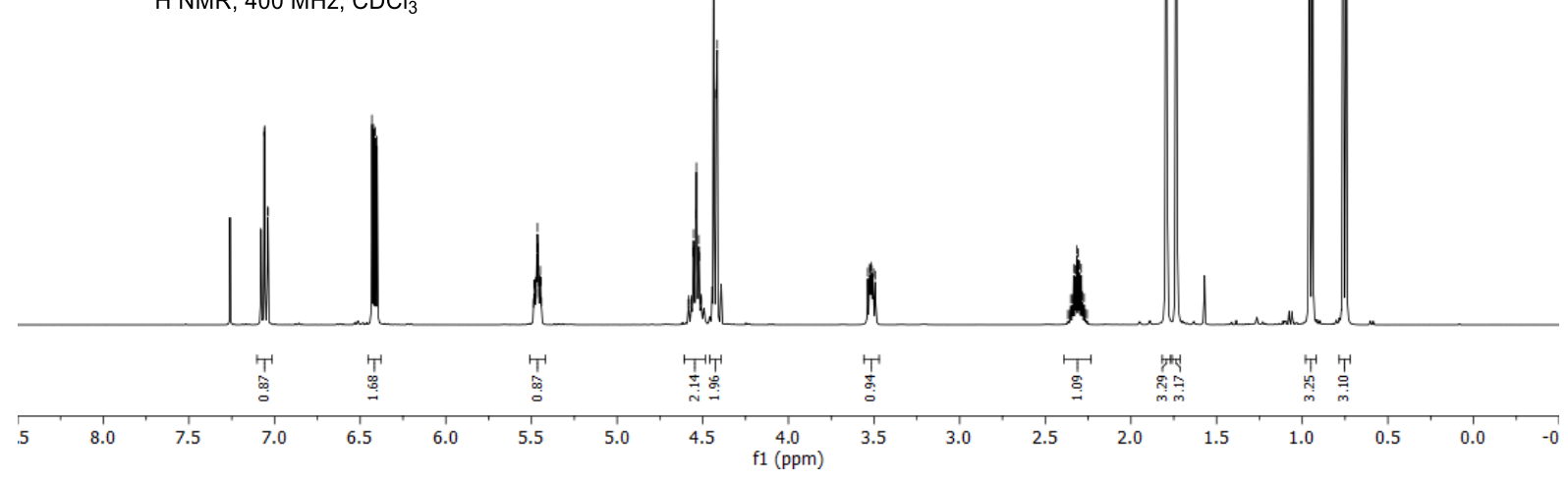

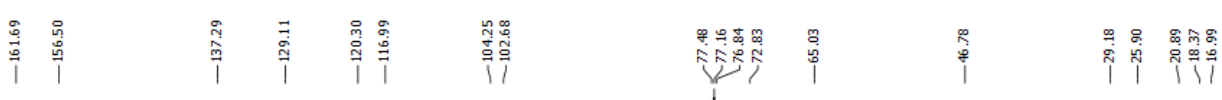

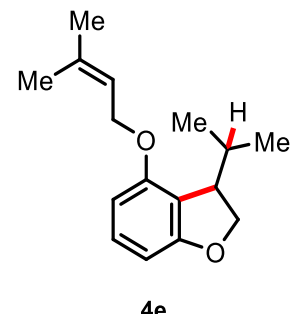

${ }^{13} \mathrm{C}$ NMR, $101 \mathrm{MHz}, \mathrm{CDCl}_{3}$

$30 \quad 170$

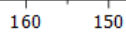

$140 \quad 130$

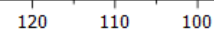

90 f1 80 


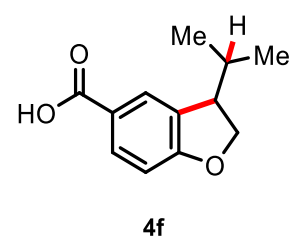

${ }^{1} \mathrm{H} \mathrm{NMR}, 400 \mathrm{MHz}, \mathrm{CDCl}_{3}$

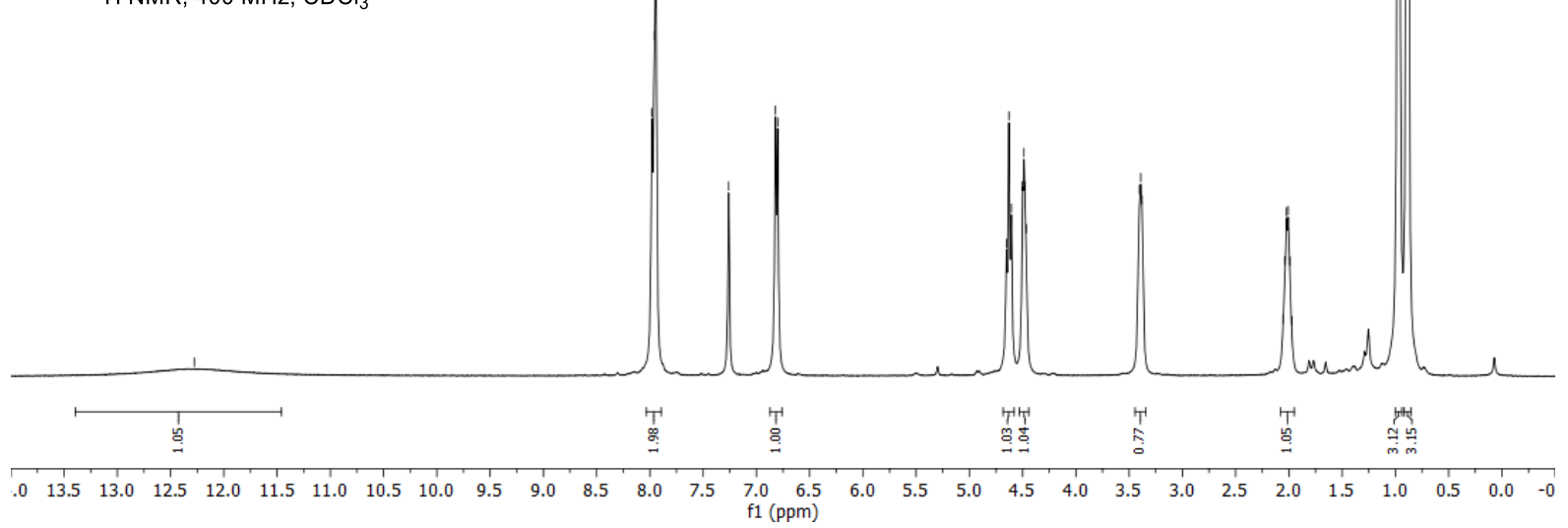

I

算等

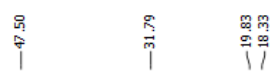

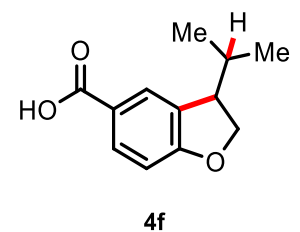

${ }^{13} \mathrm{C}$ NMR, $101 \mathrm{MHz}, \mathrm{CDCl}_{3}$
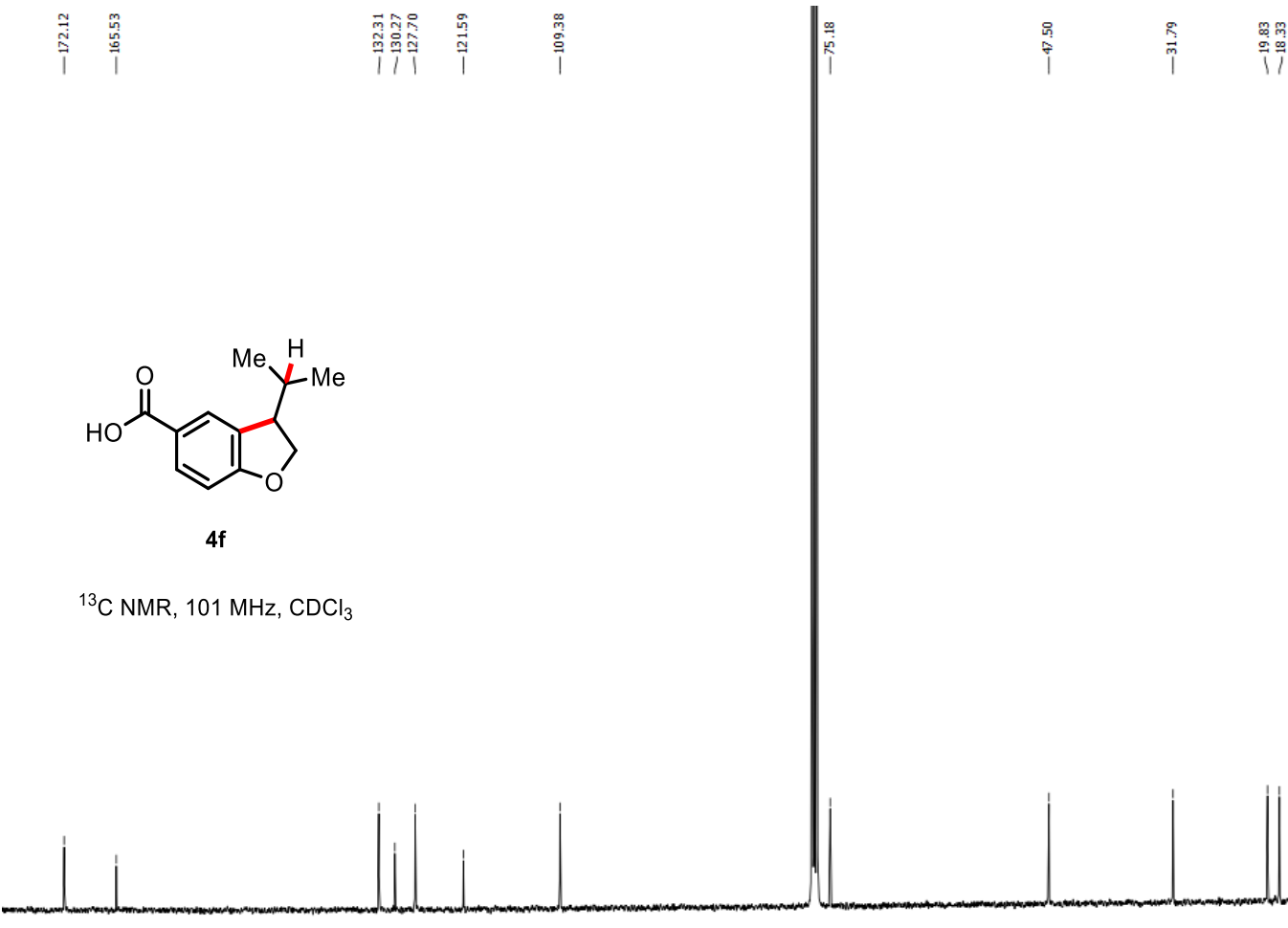

$30 \quad 170$

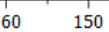

140

$90 \quad 80$

$70 \quad 60$

$20+10 \times \frac{1}{10}$ 


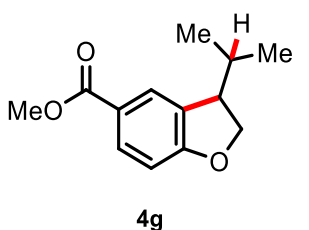

${ }^{1} \mathrm{H} \mathrm{NMR}, 400 \mathrm{MHz}, \mathrm{CDCl}_{3}$

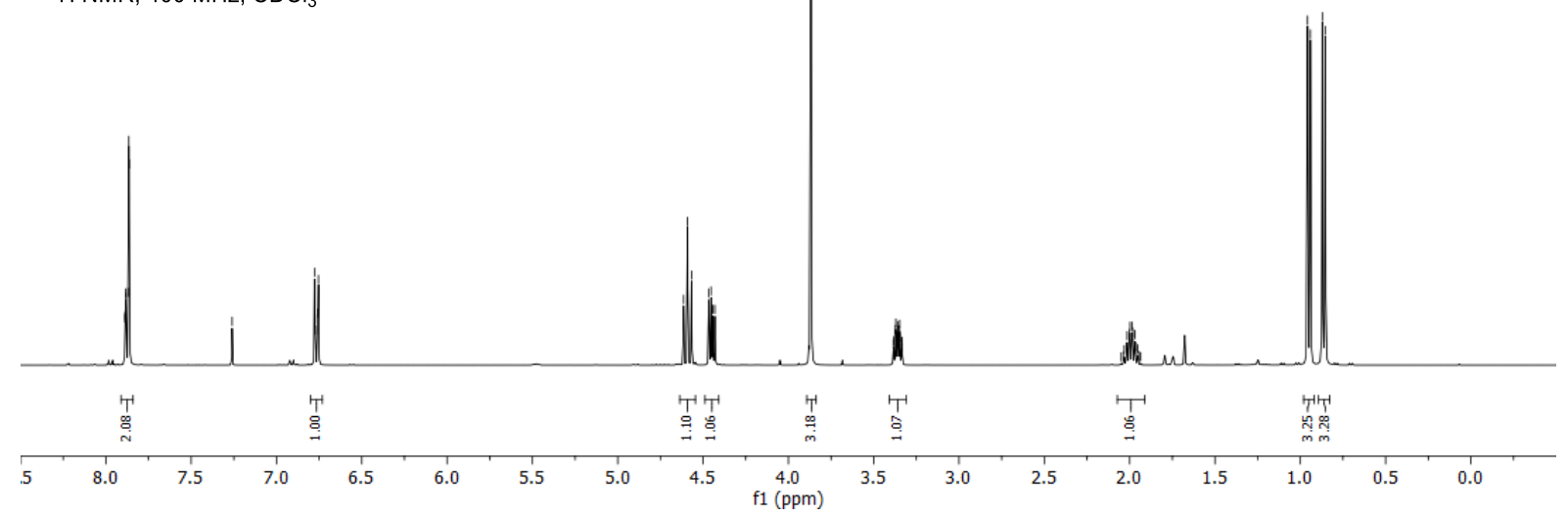

突号

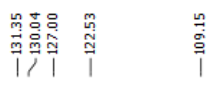

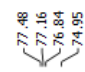

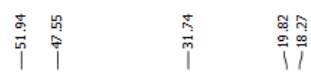

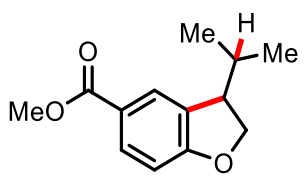

$4 \mathrm{~g}$

${ }^{13} \mathrm{C} \mathrm{NMR}, 101 \mathrm{MHz}, \mathrm{CDCl}_{3}$

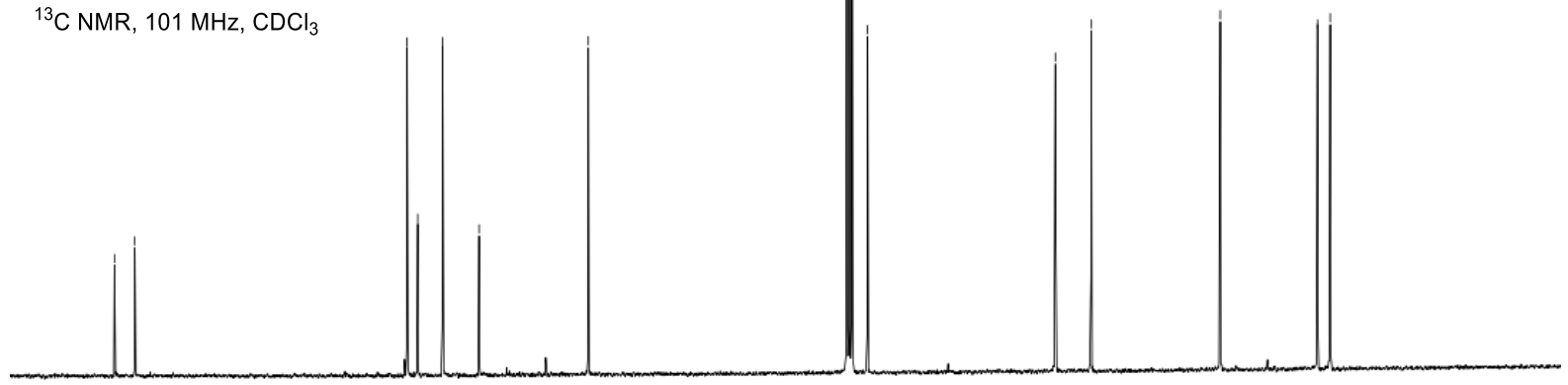

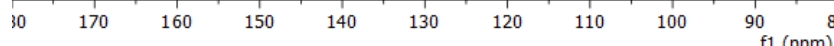

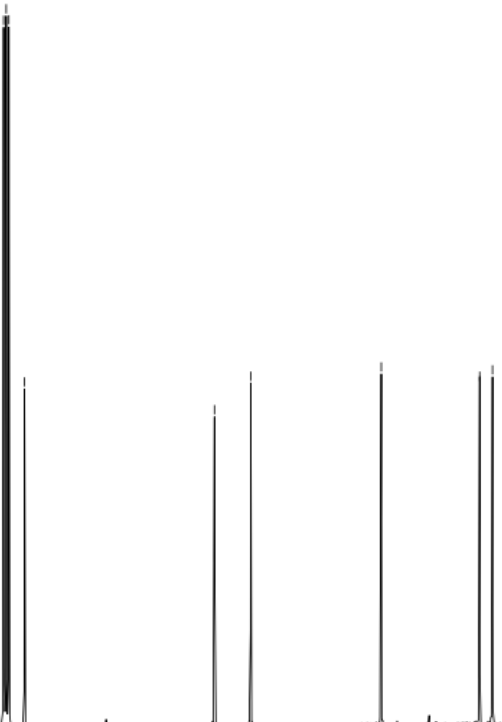




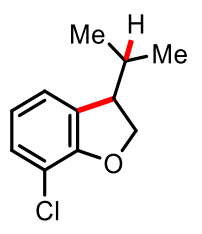

4h

${ }^{1} \mathrm{H} \mathrm{NMR}, 400 \mathrm{MHz}, \mathrm{CDCl}_{3}$

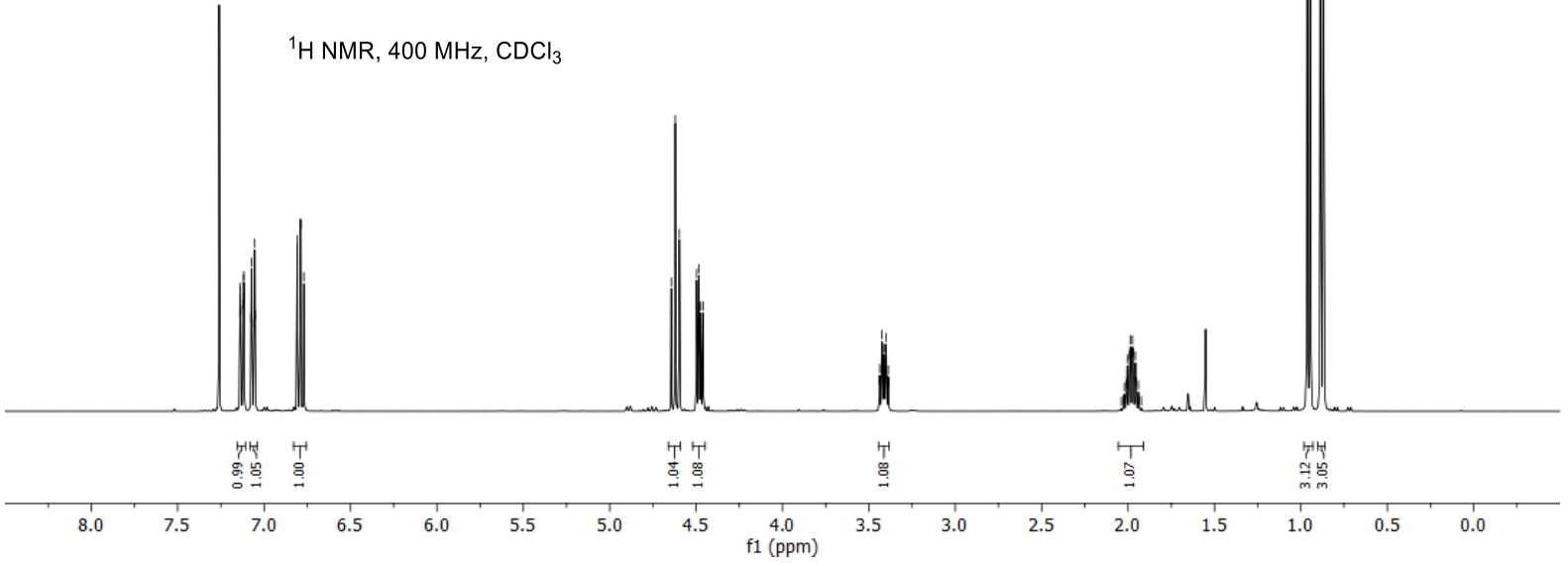

।

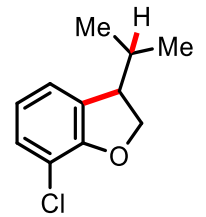

$4 h$

${ }^{13} \mathrm{C} \mathrm{NMR}, 101 \mathrm{MHz}, \mathrm{CDCl}_{3}$

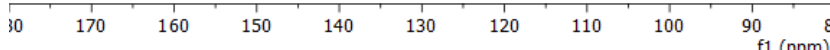
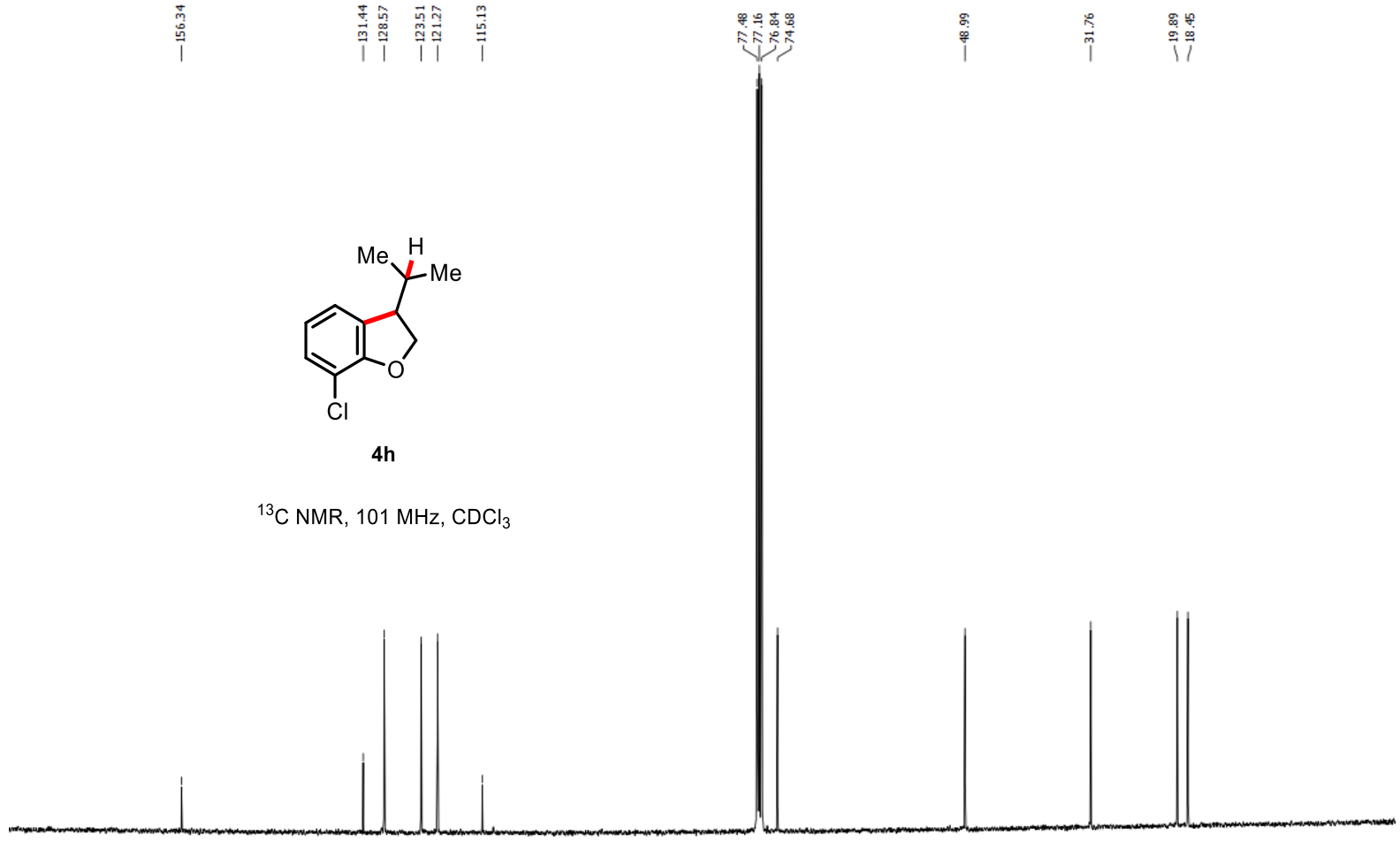

$\begin{array}{llllllllll}70 & 60 & 50 & 40 & 30 & 20 & 10 & 0 & -1\end{array}$




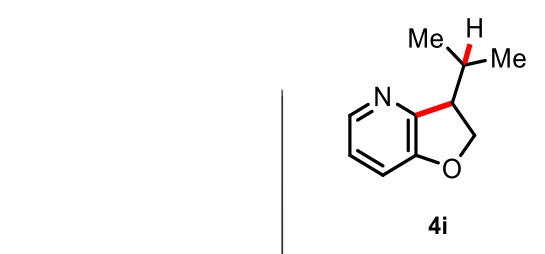

${ }^{1} \mathrm{H} \mathrm{NMR}, 400 \mathrm{MHz}, \mathrm{CDCl}_{3}$
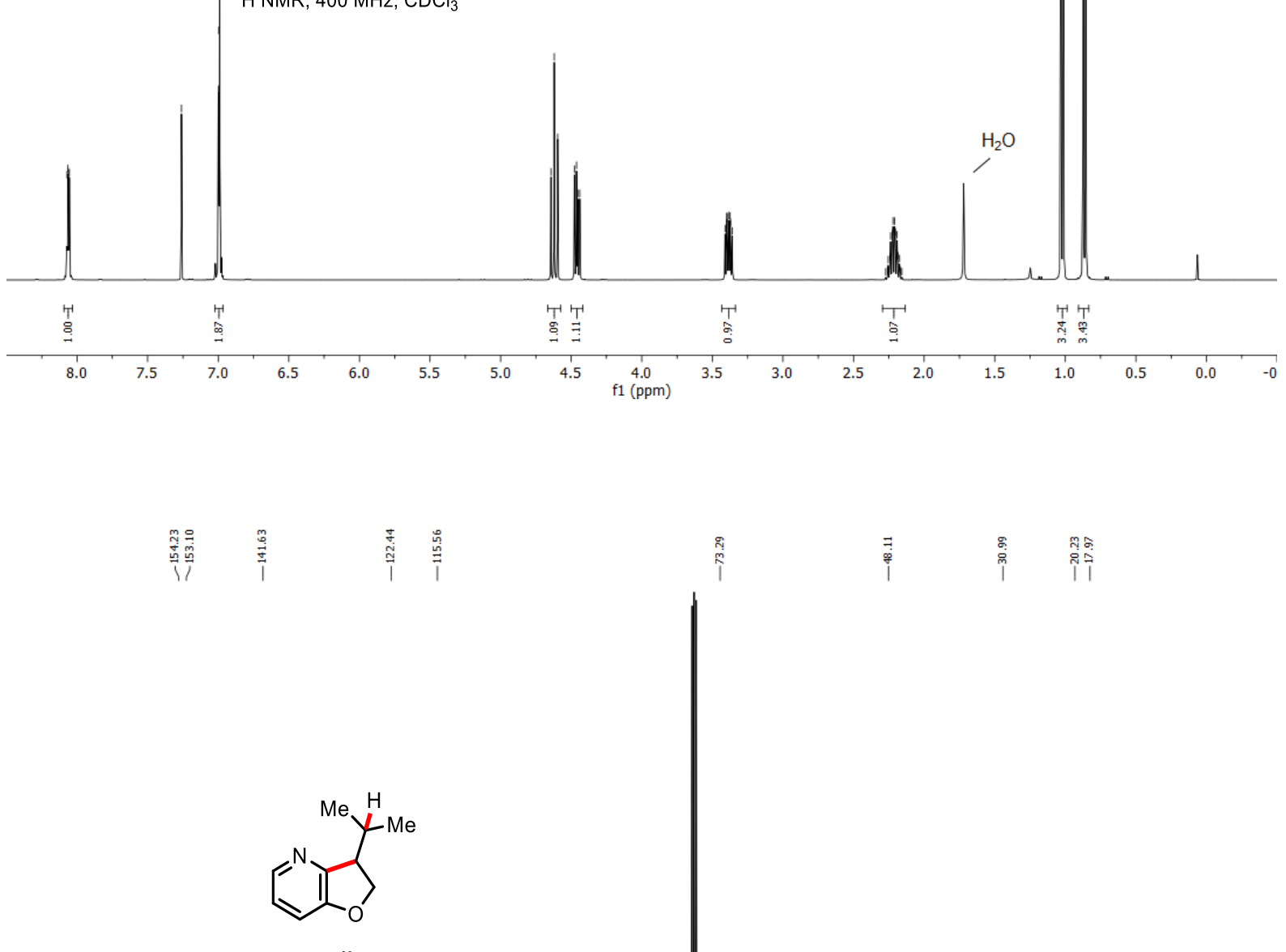

$4 i$

${ }^{13} \mathrm{C}$ NMR, $101 \mathrm{MHz}, \mathrm{CDCl}_{3}$

$30 \quad 170 \quad 160 \quad 150$ 


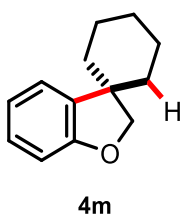

${ }^{1} \mathrm{H} \mathrm{NMR}, 400 \mathrm{MHz}, \mathrm{CDCl}_{3}$
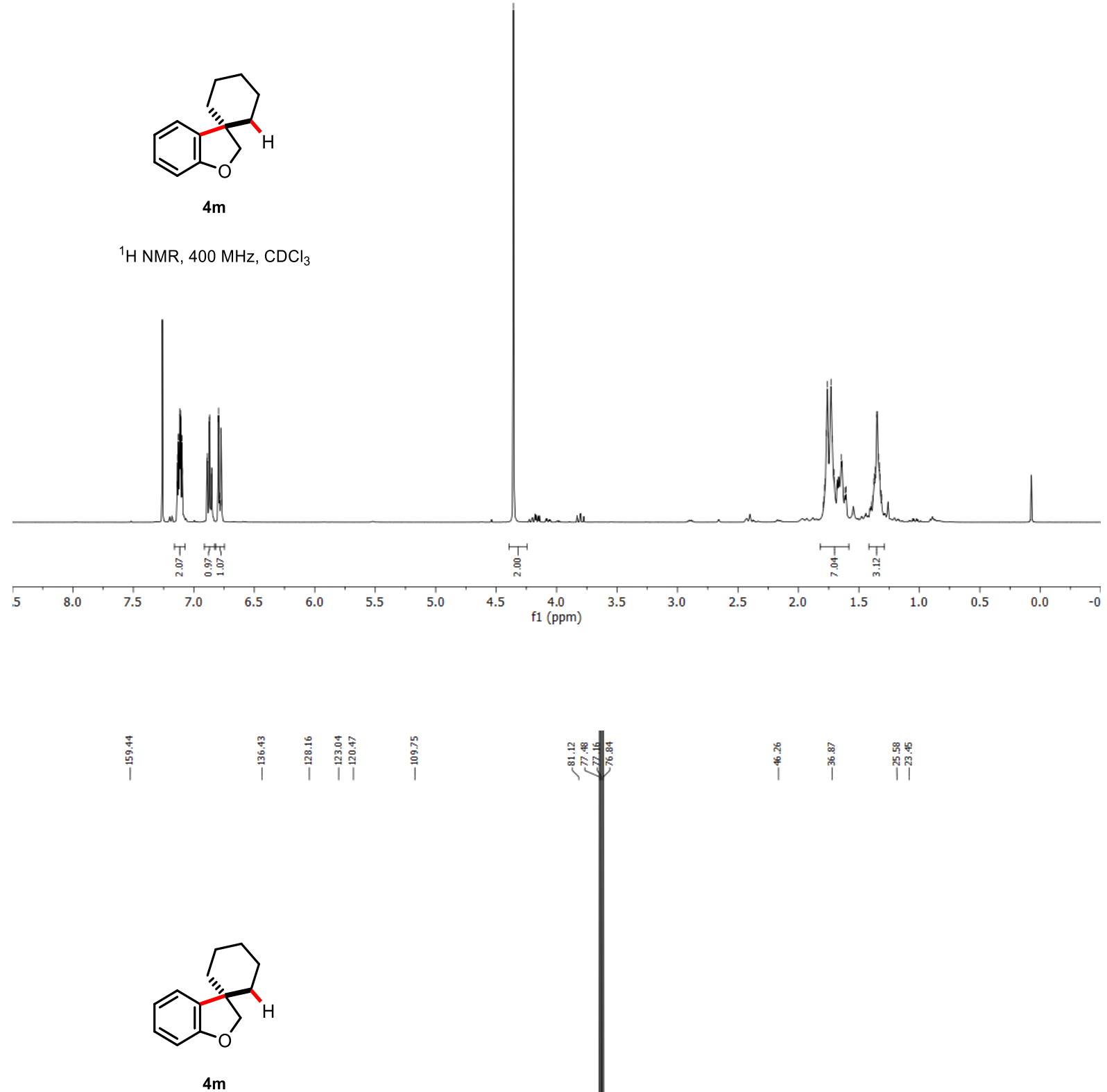

${ }^{13} \mathrm{C} \mathrm{NMR}, 101 \mathrm{MHz}, \mathrm{CDCl}_{3}$

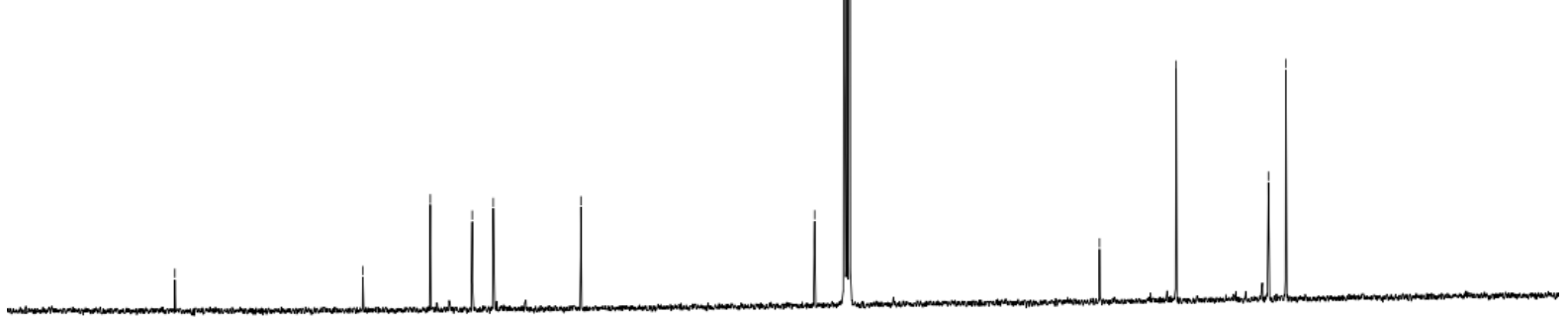

$160 \quad 150$

$140 \quad 130 \quad 120 \quad 110 \quad 100$

$90 \quad 80$ 

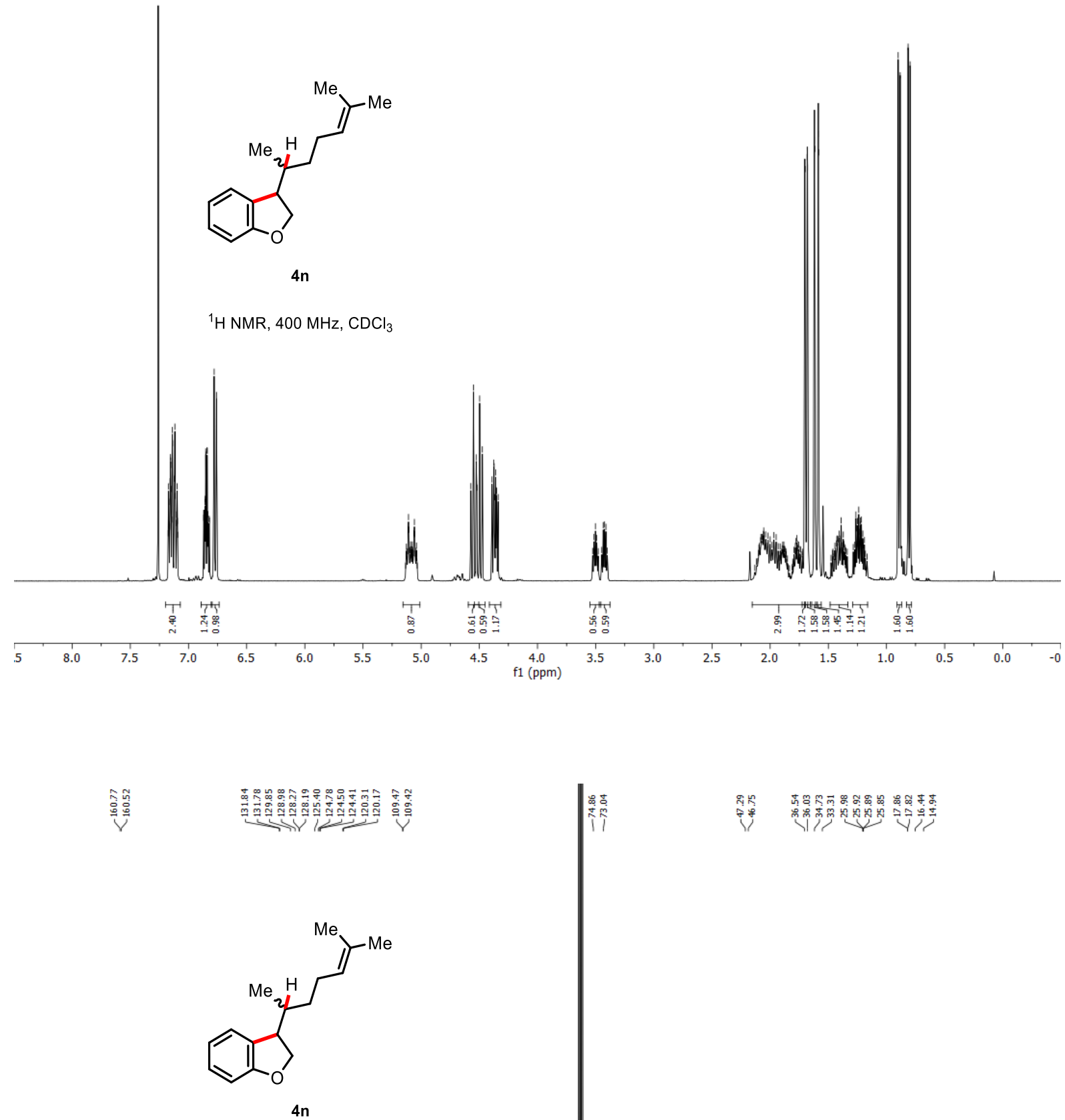

${ }^{13} \mathrm{C} \mathrm{NMR}, 101 \mathrm{MHz}, \mathrm{CDCl}_{3}$

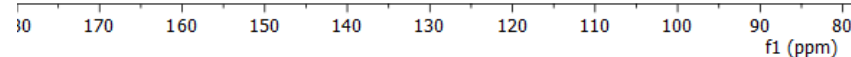

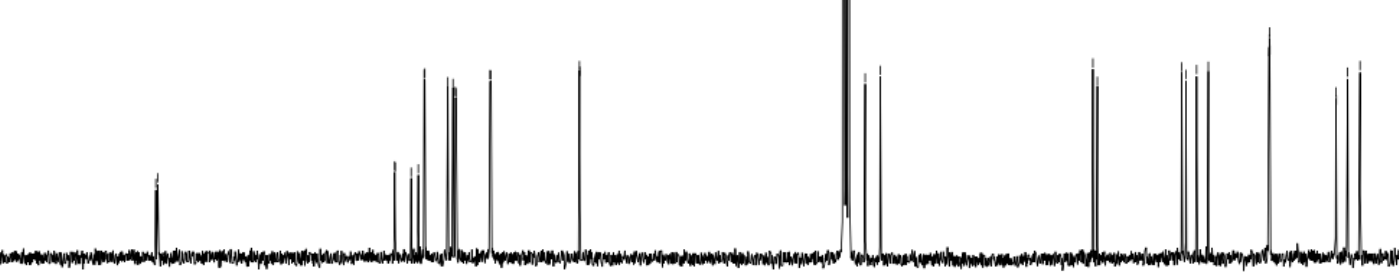




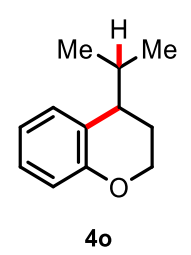

${ }^{1} \mathrm{H} \mathrm{NMR}, 400 \mathrm{MHz}, \mathrm{CDCl}_{3}$
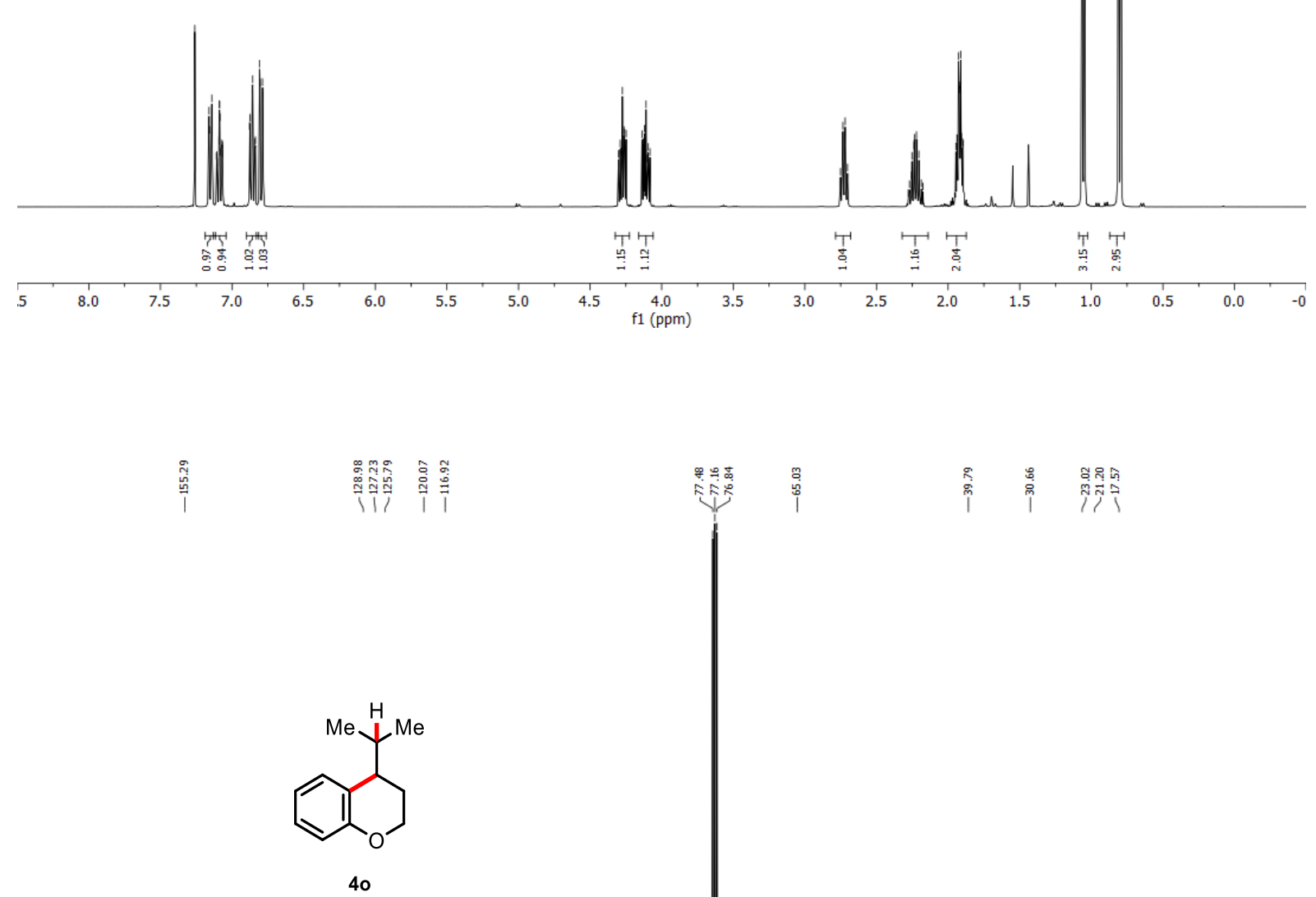

${ }^{13} \mathrm{C} \mathrm{NMR}, 101 \mathrm{MHz}, \mathrm{CDCl}_{3}$
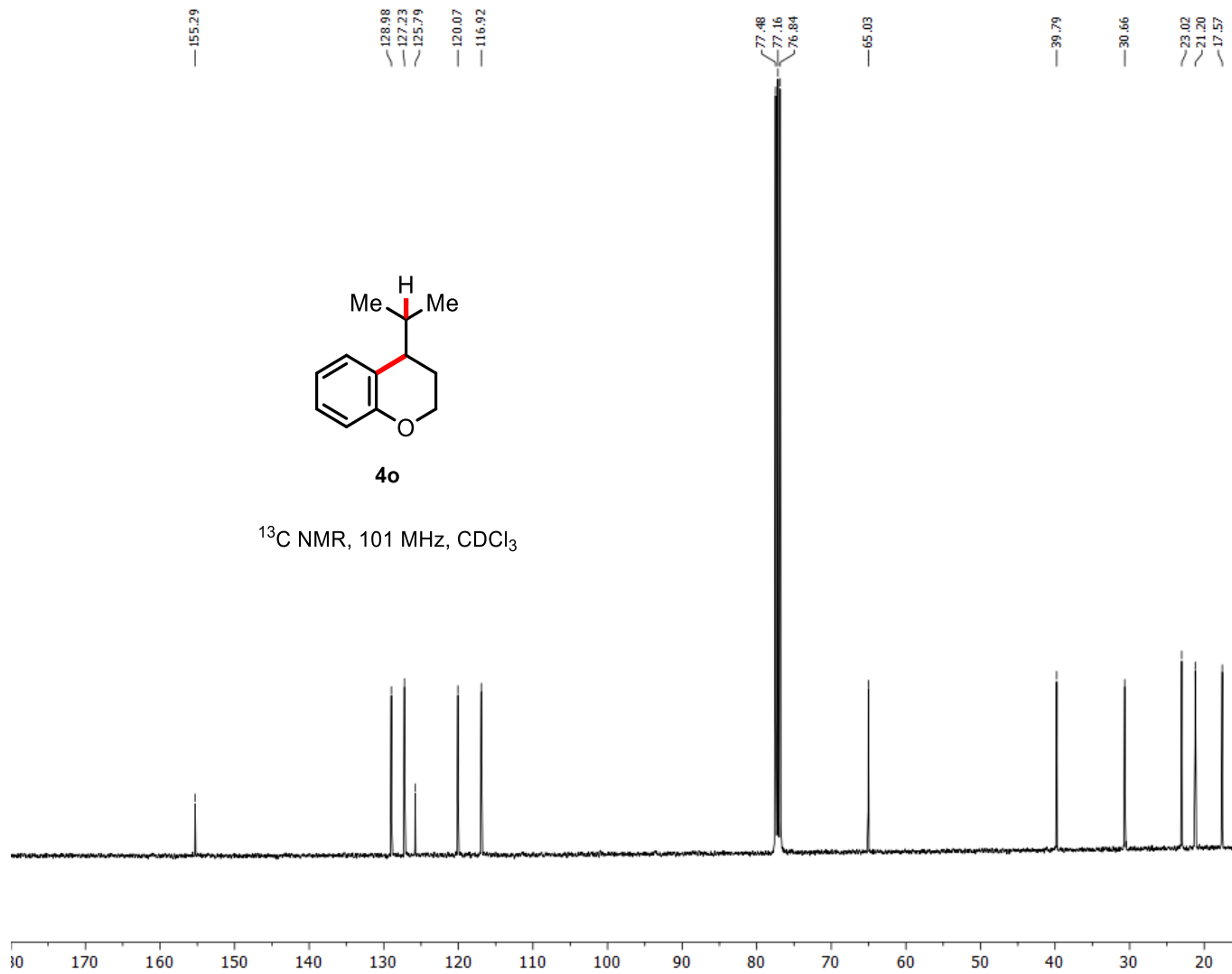

$30 \quad 170 \quad 160 \quad 150$

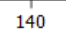

$130 \quad 120$

$90 \quad 80$ 


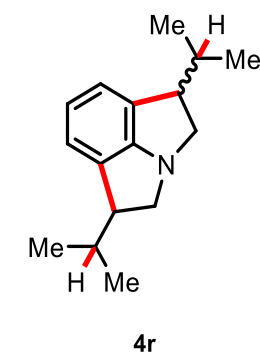

${ }^{1} \mathrm{H} \mathrm{NMR}, 400 \mathrm{MHz}, \mathrm{CDCl}_{3}$
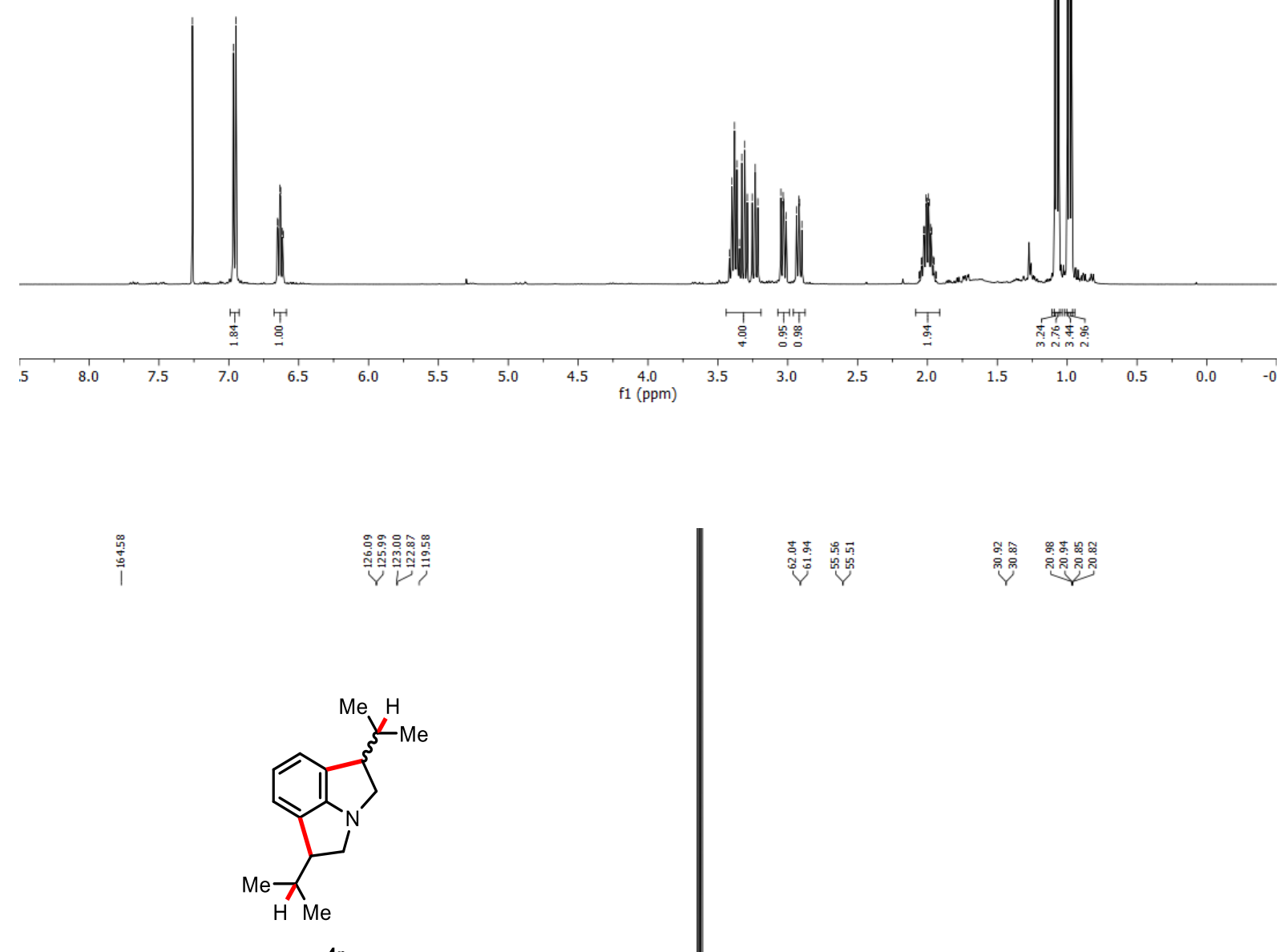

${ }^{13} \mathrm{C} \mathrm{NMR}, 101 \mathrm{MHz}, \mathrm{CDCl}_{3}$

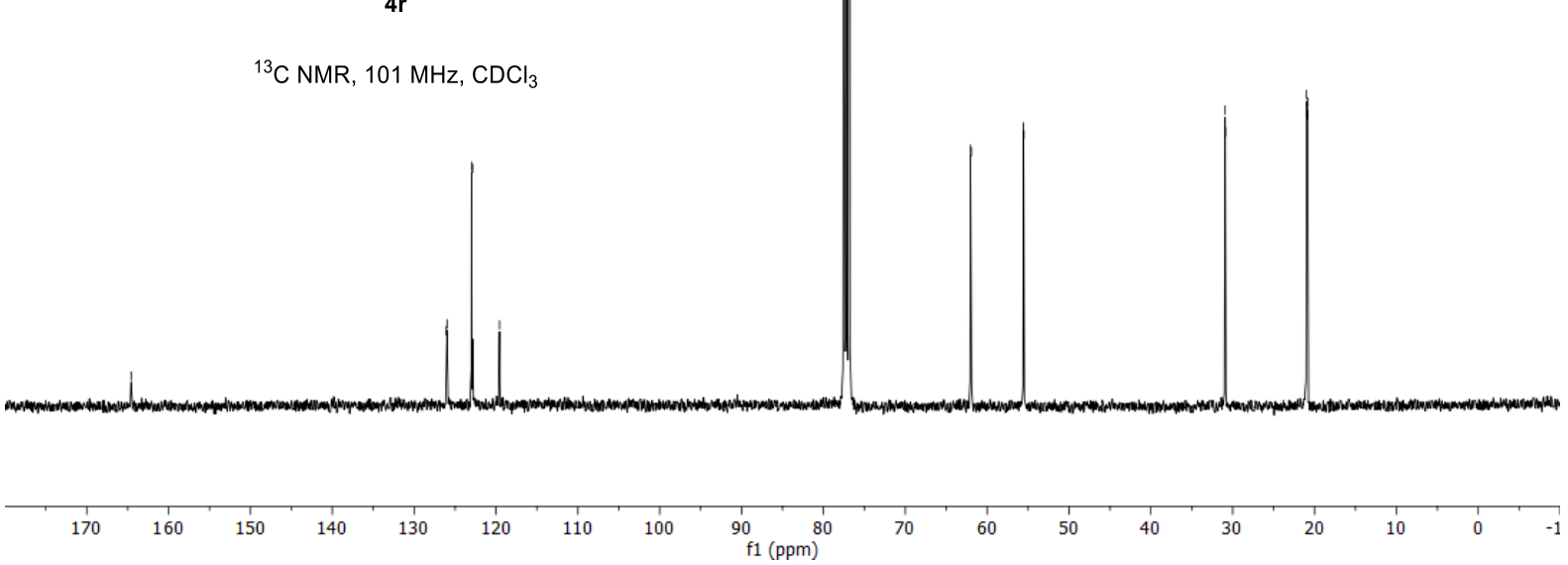

$\begin{array}{lllllllll}170 & 160 & 150 & 140 & 130 & 120 & 110 & 100 & 90 \\ & & & & & & & & \end{array}$

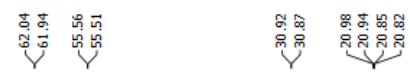

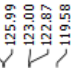

| 


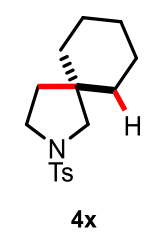

${ }^{1} \mathrm{H}$ NMR, $400 \mathrm{MHz}, \mathrm{CDCl}_{3}$
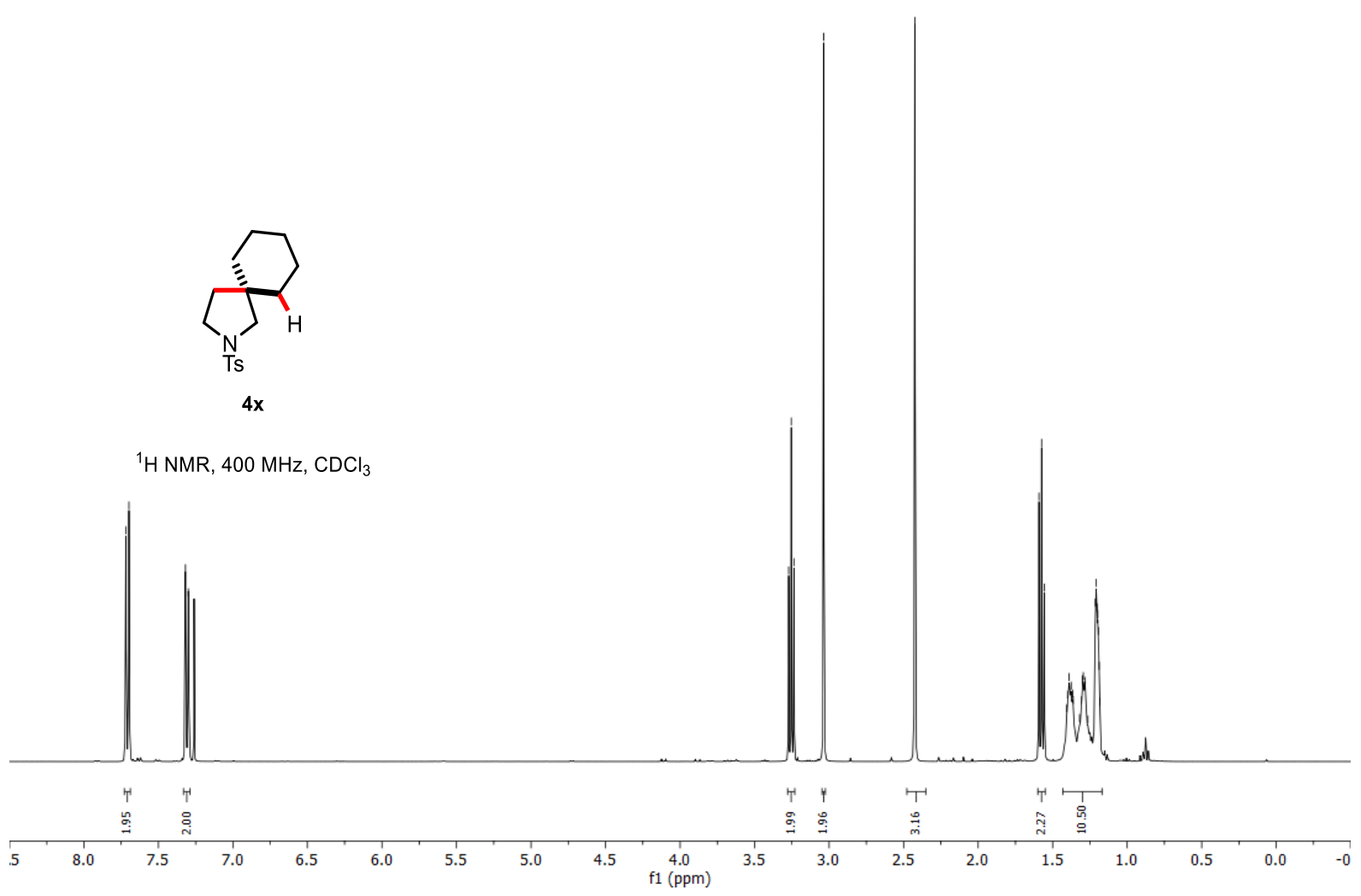

䫓

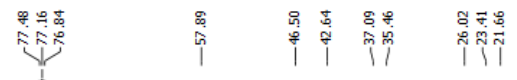

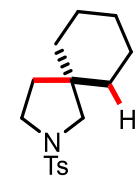

$4 \mathbf{x}$

${ }^{13} \mathrm{C} \mathrm{NMR}, 101 \mathrm{MHz}, \mathrm{CDCl}_{3}$

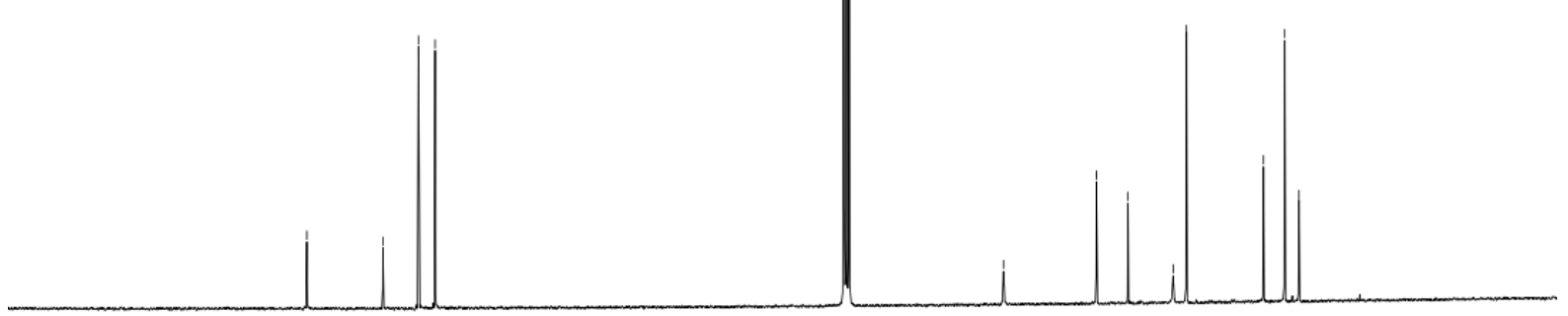

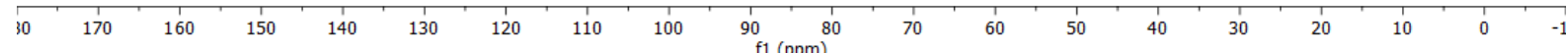




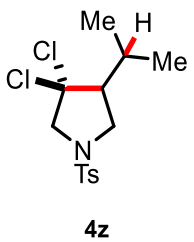

${ }^{1} \mathrm{H} \mathrm{NMR}, 400 \mathrm{MHz}, \mathrm{CDCl}_{3}$

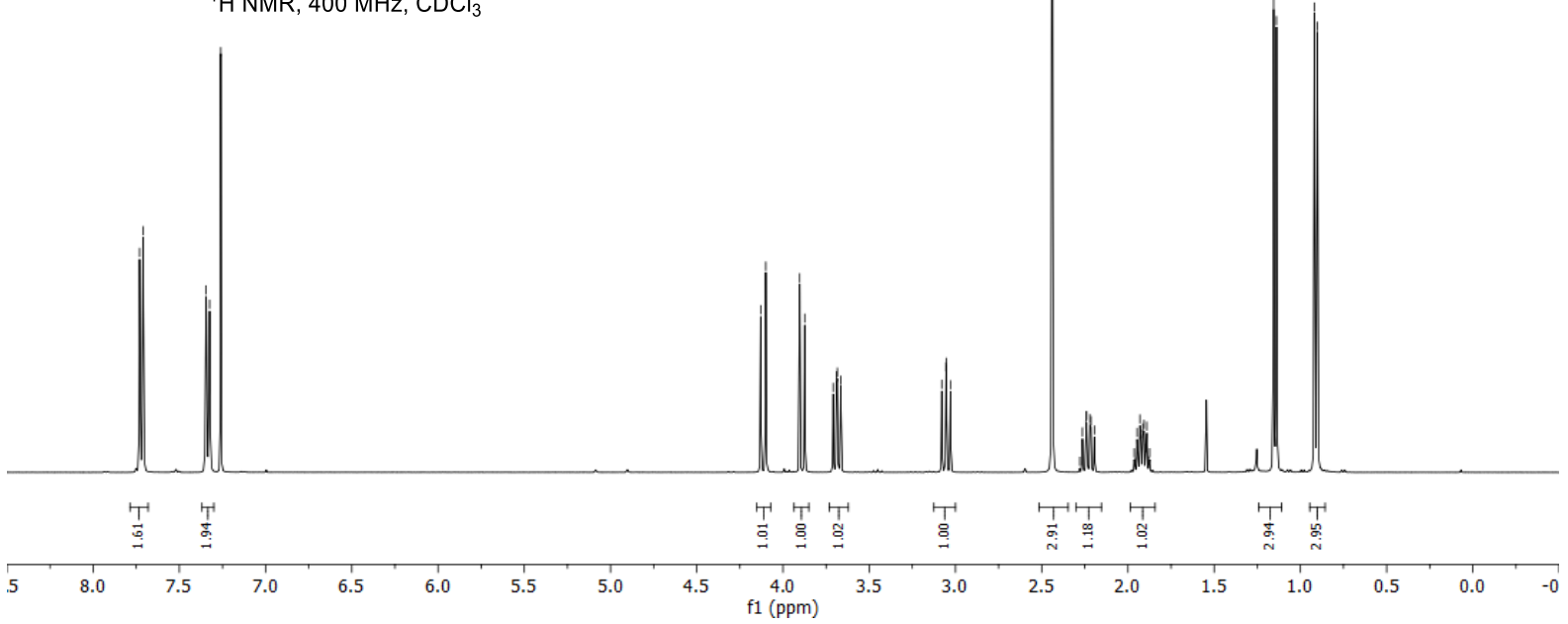

+
1

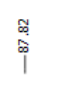

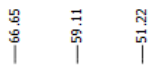

竞

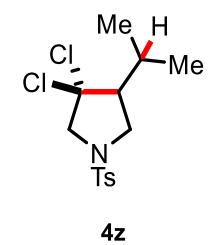

${ }^{13} \mathrm{C} \mathrm{NMR}, 101 \mathrm{MHz}, \mathrm{CDCl}_{3}$

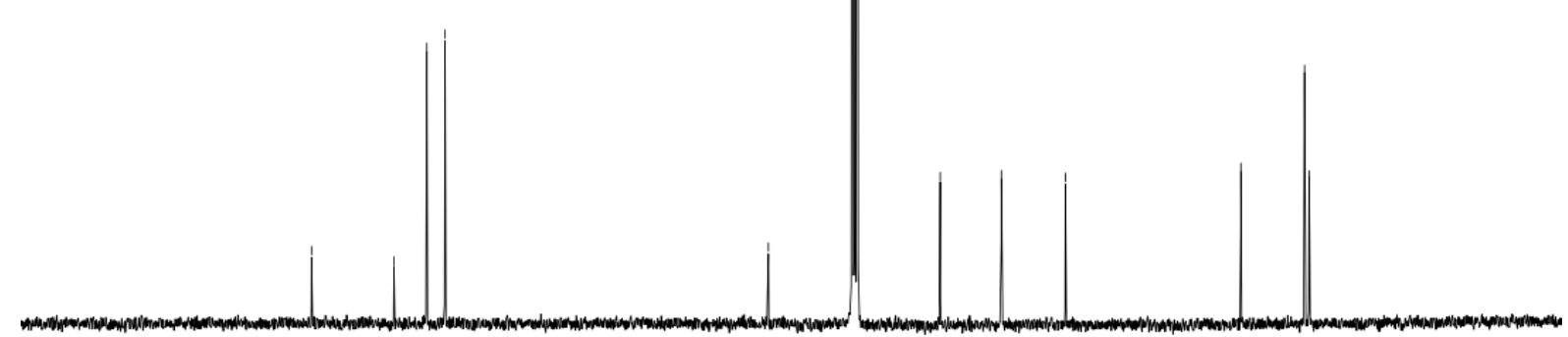

$\begin{array}{llllllllll}10 & 170 & 160 & 150 & 140 & 130 & 120 & 110 & 100 & 90 \\ & & & & & & & 1 \\ \mathrm{f} 1(\mathrm{ppm})\end{array}$ 


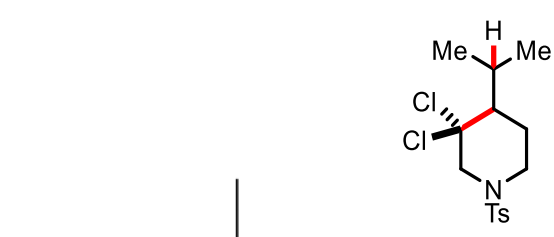

$4 z a$

${ }^{1} \mathrm{H} \mathrm{NMR}, 400 \mathrm{MHz}, \mathrm{CDCl}_{3}$

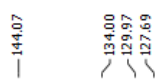<smiles>CC(C)C1CCN(S)CC1(Cl)Cl</smiles>

4za

${ }^{13} \mathrm{C} \mathrm{NMR}, 101 \mathrm{MHz}, \mathrm{CDCl}_{3}$

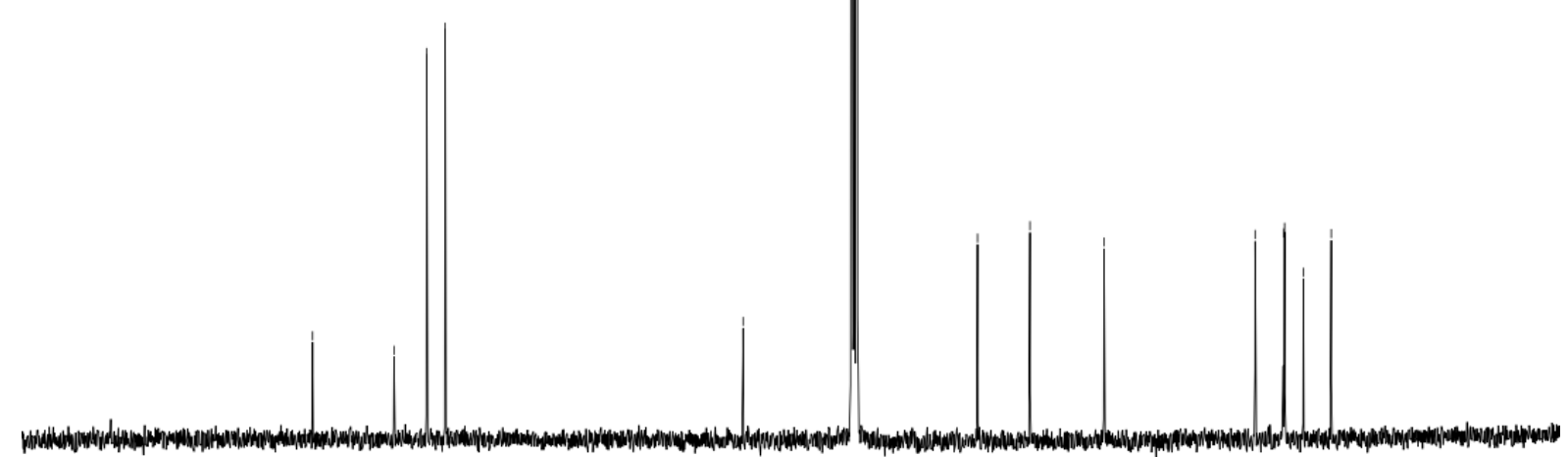

\begin{tabular}{rrrrrrrrrr}
\hline 10 & 170 & 160 & 150 & 140 & 130 & 120 & 110 & 100 & 90 \\
$\mathrm{f} 1(\mathrm{ppm})$ & 80
\end{tabular} 